\title{
Psychedelics in Psychiatry: Neuroplastic, Immunomodulatory, and Neurotransmitter Mechanisms $^{\text {[ }}$
}

\author{
Antonio Inserra, Danilo De Gregorio, and Gabriella Gobbi \\ Neurobiological Psychiatry Unit, Department of Psychiatry, McGill University, Montreal, Quebec, Canada
}

Abstract ....................................................................205

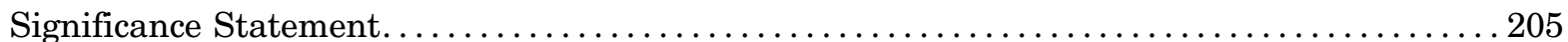

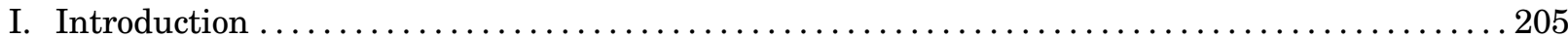

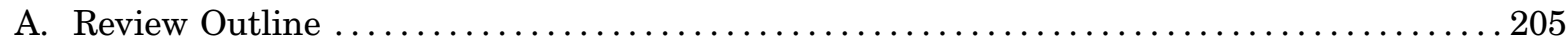

B. Psychiatric Disorders and the Need for Novel Pharmacotherapies ................206

C. Psychedelic Compounds as Novel Therapeutics in Psychiatry: Overview and

Comparison with Current Available Treatments ......................... 206

D. Classical or Serotonergic Psychedelics versus Nonclassical Psychedelics: Definition ...... 208

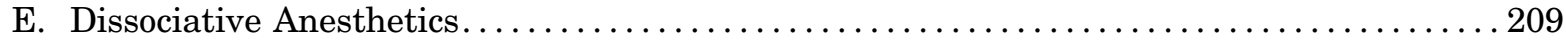

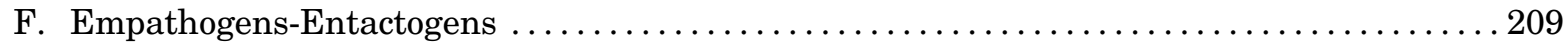

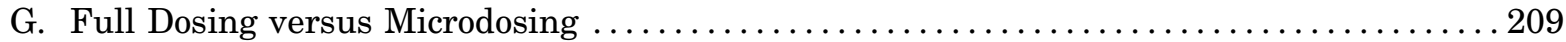

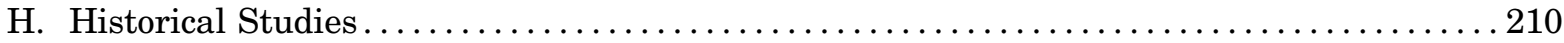

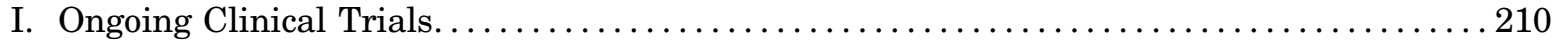

II. Psychedelic Compounds as Neuroplastic Agents ............................. 211

A. Neuroplasticity Impairments in Psychiatric Disorders $\ldots \ldots \ldots \ldots \ldots \ldots \ldots \ldots \ldots \ldots 211$

B. Effects of Psychedelic Compounds on Neuronal and Synaptic Plasticity............. 223

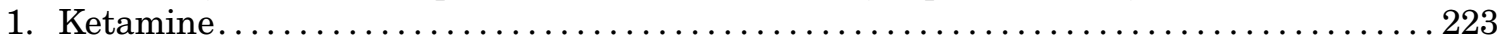

2. N,N-Dimethyltryptamine, 5-Methoxy-N,N-dimethyltryptamine, and Ayahuasca . . . . 224

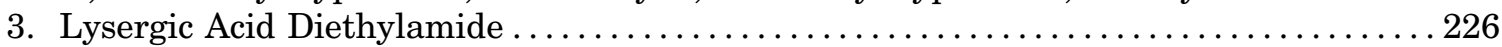

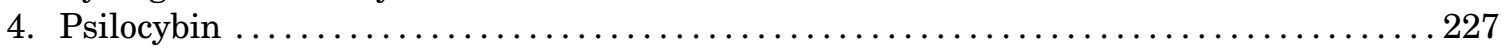

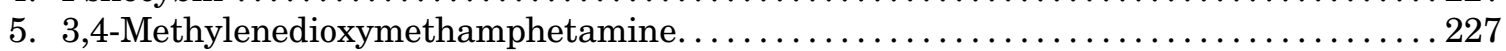

III. Psychedelic Compounds as Immunomodulatory and Anti-Inflammatory Agents......... 227

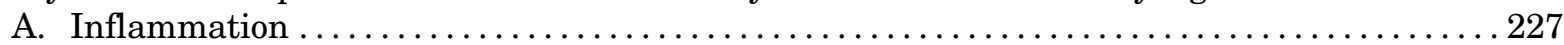

B. Inflammation-Induced Psychiatric Symptoms .............................. 228

C. Anti-Inflammatory Therapies in the Treatment of Psychiatric Disorders.............228

D. Immunomodulatory and Anti-Inflammatory Pathways Activated by Psychedelics ....... 228

1. N,N-Dimethyltryptamine, 5-Methoxy-N,N-dimethyltryptamine, and Ayahuasca . . . . 230

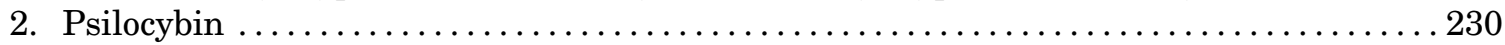

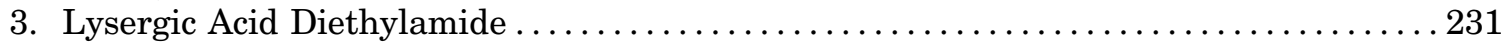

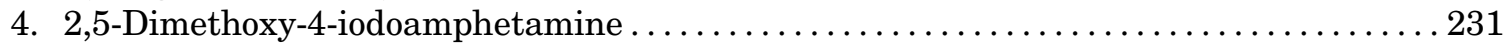

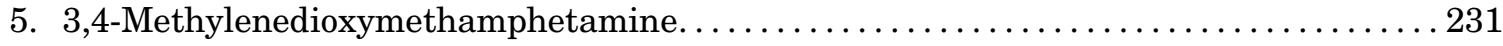

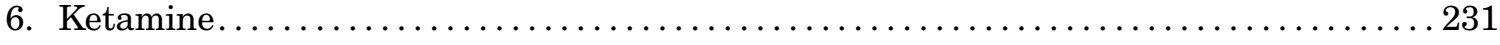

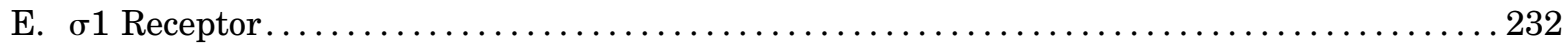

\footnotetext{
Address correspondence to: Gabriella Gobbi, Neurobiological Psychiatry Unit, Department of Psychiatry, McGill University, 1033, Av. des Pins Ouest, Montreal, QC H3A 1A1, Canada. E-mail: gabriella.gobbi@mcgill.ca

This work was supported by the Canadian Institute for Health Research (CIHR) [Grant \# 436986] and by the Réseau Québécois sur le Suicide, les Troubles de l'Humeur et les Troubles Associés (RQSHA) [Grant \# 268065]. D.D.G. is recipient of Fond Recherche Québec-Santé (FRQS) and Canadian Institutes for Health Research (CIHR) postdoctoral fellowships.

G.G. is a consultant for Diamond Therapeutic Inc. The other authors declare no potential conflicts of interest with respect to the research, authorship, and/or publication of this article.

\$This article has supplemental material available at pharmrev.aspetjournals.org.

https://doi.org/10.1124/pharmrev.120.000056.
} 
IV. Psychedelic Compounds as Modulators of Neurotransmitter Systems................. 232

A. Neurotransmitter Dysfunctions in Psychiatric Disorders . . . . . . . . . . . . . . . . 232

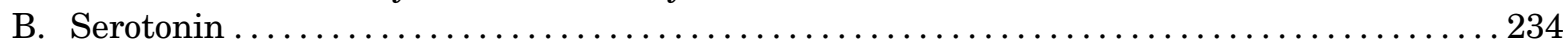

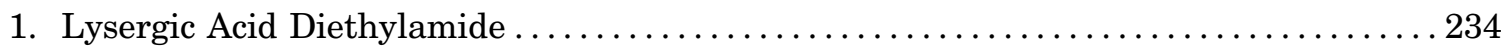

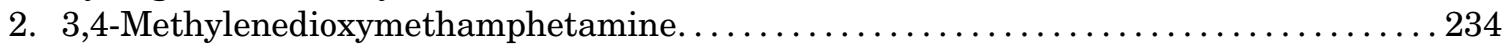

3. N,N-Dimethyltryptamine, 5-Methoxy-N,N-dimethyltryptamine, and Ayahuasca . ... . 235

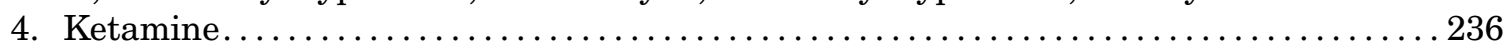

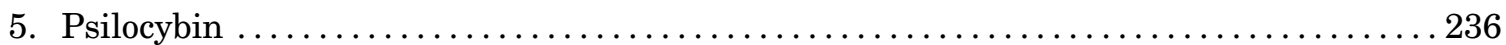

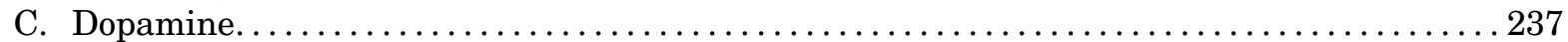

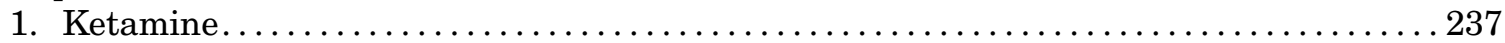

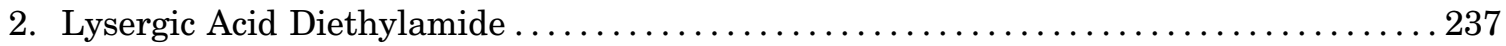

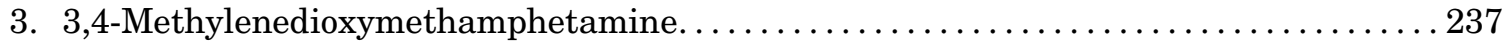

4. N,N-Dimethyltryptamine, 5-Methoxy-N,N-dimethyltryptamine, and Ayahuasca . . ... 238

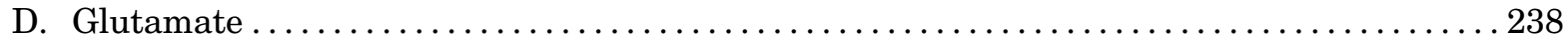

1. Lysergic Acid Diethylamide ...................................... 238

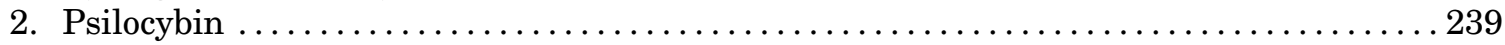

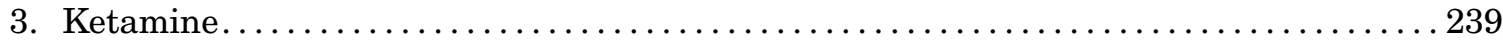

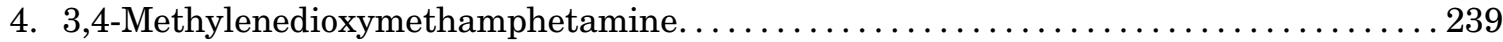

5. N,N-Dimethyltryptamine, Ayahuasca, 5-Methoxy-N,N-dimethyltryptamine ........ 240

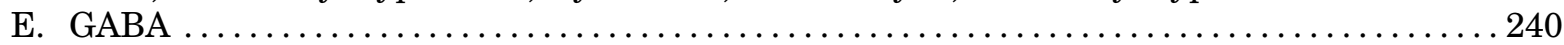

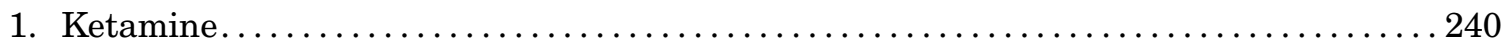

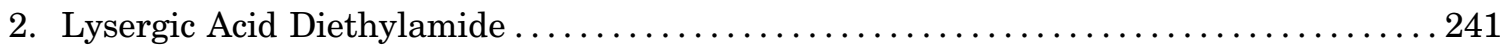

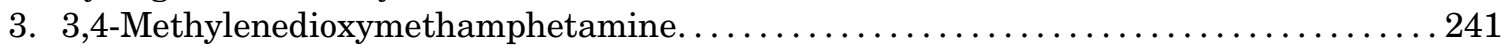

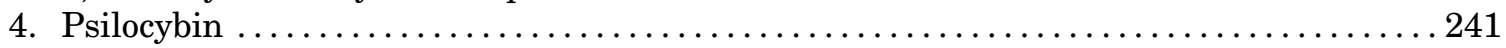

5. N,N-Dimethyltryptamine, 5-Methoxy-N,N-dimethyltryptamine, Ayahuasca . ....... 241

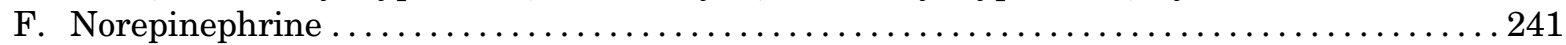

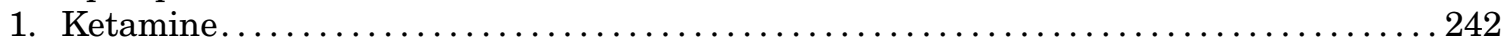

\begin{abstract}
ABBREVIATIONS: ACC, anterior cingulate cortex; ACTH, adrenocorticotropic hormone; AD, Alzheimer's disease; ADRA, $\alpha$ adrenergic receptor; ADRB, $\beta$ adrenergic receptor; AIDS, acquired immunodeficiency syndrome; ALD-52, 1-acetyl-LSD; ALT, alternative lengthening of telomeres; AMPA, $\alpha$-amino-3-hydroxy-5-methyl-4-isoxazolepropionic acid; AN, anorexia nervosa; ANIA3, activity and neurotransmitter-induced early gene 3; AR, androgen receptor; ASD, autism spectrum disorder; AUD, alcohol use disorder; BD, bipolar disorder; BDNF, brainderived neurotrophic factor; 1B-LSD, 1-butanoyl-LSD; CA, cornu ammonis; CACNA1A, voltage-dependent P/Q-type calcium channel subunit alpha-1A; CAMK2, calcium/calmodulin-dependent protein kinase 2; CCL5, C-C motif chemokine 5; CEBPB, CCAAT/enhancer-binding protein beta; cFOS, proto-oncogene c-Fos; CHRM, muscarinic acetylcholine receptor M2; COMT, catechol O-methyltransferase; CORT, cortisol; CREB, cAMP response element-binding protein; CRF, corticotropin-releasing factor; CSTC, cortico-striato-thalamo-cortical; CX3CL1, fractalkine CXCL10 C-X-C motif chemokine 10; DA, dopamine; DAT, dopamine transporter; DHEA, dehydroepiandrosterone; D2L, dopamine 2 receptor (long splice variant); DMN, default mode network; DMT, $N, N$-dimethyltryptamine; DOI, 2,5-dimethoxy-4-iodoamphetamine; DRN, dorsal raphe nucleus; EAAT, excitatory amino acid transporter; EEG, electroencephalogram; EGR, early growth response; EPAC1, rap guanine nucleotide exchange factor 3; ERK, extracellular signal-regulated kinase; FDA, Food and Drug Administration; fMRI, functional magnetic resonance imaging; FPTC, frontoparietal task control network; GAT, GABA transporter; GABR, gamma-aminobutyric acid type B receptor; $\mathrm{GH}$, growth hormone; GLU, glutamate; GPCR, G protein-coupled receptor; 5 -GSyl- $\alpha$-MeDA, 5 -(glutathion-Syl)- $\alpha$-methyldopamine; 6 -HOMDMA, 2-hydroxy-4,5(methylenedioxy)methamphetamine; HPA, hypothalamic-pituitary-adrenal axis; HRH, histamine receptor; 5-HT, serotonin; I1, imidazoline receptor 1; ICAM, intercellular adhesion molecule; IDO, indoleamine 2,3-dioxygenase; IFN- $\gamma$, interferon- $\gamma$; IKB, inhibitor of kB kinase; IL, interleukin; iNOS, inducible nitric oxide synthase; IP3R, inositol trisphosphate receptor; KROX20, early growth response protein 2; LC, locus coeruleus; LSD, lysergic acid diethylamide; M3, muscarinic receptor subtype 3; M5, muscarinic receptor subtype 5; MAOI, monoamine oxidase inhibitor; MAPK1, mitogen-activated protein kinase 1; MCI, mild cognitive impairment; MCP1, monocyte chemoattractant protein 1; MDA, 3,4-methylenedioxyamphetamine; MDD, major depressive disorder; MDMA, 3,4-methylenedioxymethamphetamine; 5-MeO-DMT, 5-methoxy- $N, N$-dimethyltryptamine; mGluR, metabotropic glutamate receptor; MKP1, mitogen-activated protein kinase phosphatase; MOR, $\mu$-opioid receptor; $\mathrm{mPFC}$, medial prefrontal cortex; mTOR, mammalian target of rapamycin; NAc, nucleus accumbens; NCT, ClinicalTrials.gov identifier; NE, norepinephrine; NET, norepinephrine transporter; NFAT, nuclear factor of activated Tcells; NF-kB, nuclear factor кB; NGF, nerve growth factor; NMDA, $N$-methyl-D-aspartate; NOR1, neuron-derived orphan receptor-1; NR, $N$-methyl D-aspartate receptor; NTRK2, neurotrophic receptor tyrosine kinase 2; OCD, obsessive-compulsive disorder; OPRD1, delta-type opioid receptor; OPRK1, kappa-type opioid receptor; OPRM1, mu-type opioid receptor; OT, oxytocin; OUD, opioid use disorder; PCC, posterior cingulate cortex; PCP, phencyclidine; PFC, prefrontal cortex; PKA, protein kinase A; PKC, protein kinase C; PLC, phospholipase C; 1P-LSD, 1-propanoyl-LSD; PSD, postsynaptic density; PTSD, post-traumatic stress disorder; PV, parvalbumin; RCT, randomized controlled trial; REM, rapid eye movement; RT, reticular thalamus; SCZ, schizophrenia; SERT, serotonin transporter; SLC6A13, sodium- and chloridedependent GABA transporter 2; SLC6A2, sodium-dependent noradrenaline transporter; SNRI, serotonin-norepinephrine reuptake inhibitor; S1R, $\sigma 1$ receptor; S2R, $\sigma 2$ receptor; SGK, serine/threonine-protein kinase Sgk1; SSRI, selective serotonin reuptake inhibitor; TAAR, trace amine-associated receptor; Th, T-helper; TLR, toll-like receptor; TNF- $\alpha$, tumor necrosis factor- $\alpha$; TRD, treatment resistant depression; TSH, thyroid stimulating hormone; TrkB, tyrosine receptor kinase B; VCAM, vascular cell adhesion protein; VMAT2, vesicular monoamine transporter 2; VTA, ventral tegmental area; 5-HTR, serotonin receptor; 5-HTTLPR, serotonin transporter-linked polymorphic region.
\end{abstract}




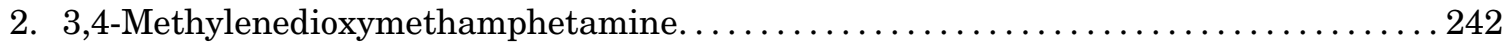

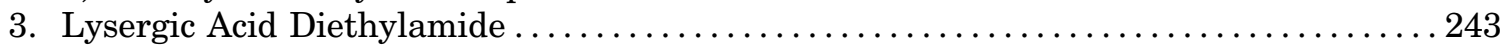

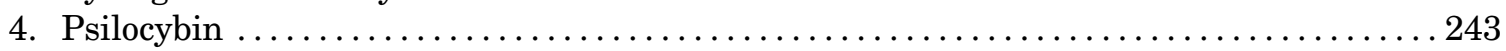

5. N,N-Dimethyltryptamine, 5-Methoxy-N,N-dimethyltryptamine, Ayahuasca . . . . . . . 243

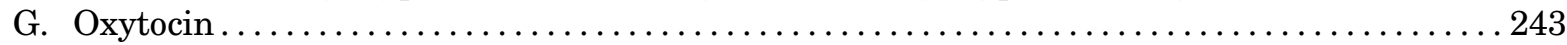

V. Biased Signaling, Biased Phosphoproteomics, and Psychedelic Compounds .............. 244

A. Role of Biased Signaling in Pharmacology and Psychedelic-Induced Biased Signaling ... 244

B. Psychedelics and Homo- and Heteroreceptor Complexes........................... 245

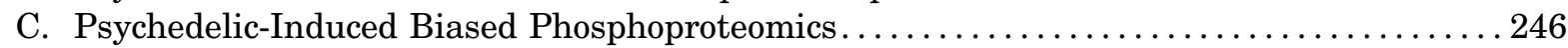

VI. Going Beyond Receptors: Neuronal Circuits Activated by Psychedelic Drugs.............. 247

A. Effects on the Default Mode Network Hub Functioning, and the "Reset" Model ........ 247

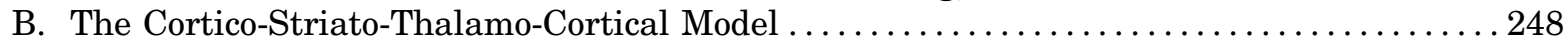

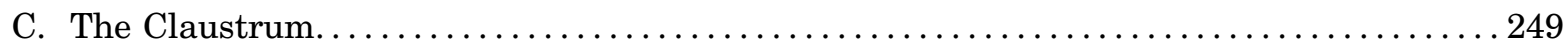

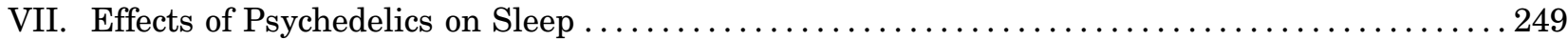

VIII. Long-Term Neurobiological and Psychological Effects of Psychedelic Compounds .......... 250

A. N,N-Dimethyltryptamine, 5-Methoxy-N,N-dimethyltryptamine, and Ayahuasca. ...... 250

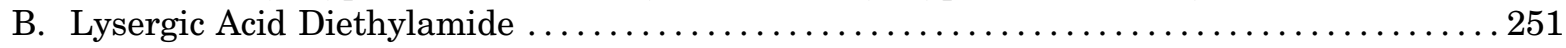

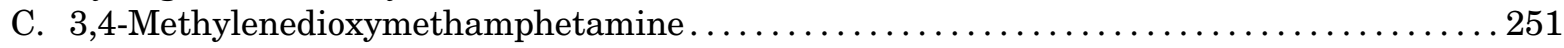

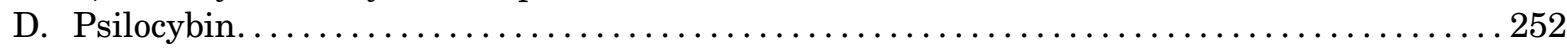

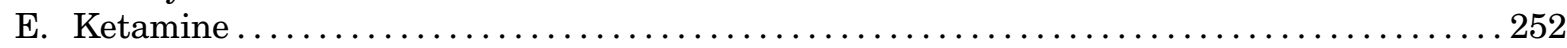

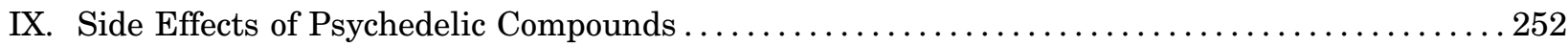

A. Acute Side Effects Encountered in Clinical Trials ........................... 253

1. Acute Physical Side Effects Encountered in Clinical Trials....................253

2. Acute Psychological Side Effects Encountered in Clinical Trials ................. 253

B. Acute Side Effects Encountered in Uncontrolled Settings . . . . . . . . . . . . . . . 253

1. Risk of Switch to Mania in Patients with Bipolar Disorder.................. 254

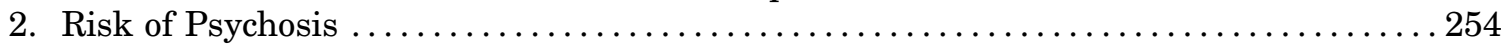

3. Acute Severe Physical Side Effects Encountered in Uncontrolled Settings .......... 254

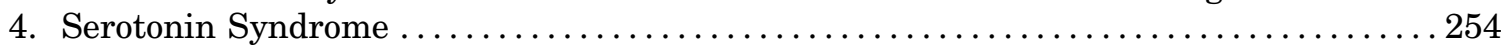

5. 3,4-Methylenedioxymethamphetamine Neurotoxicity. ................... 255

C. Abuse Potential and Potential Long-Term Side Effects of Psychedelic Compounds ....... 255

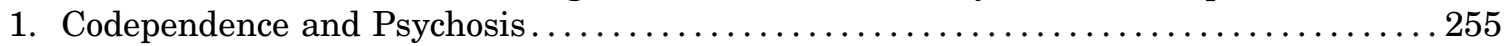

2. Abuse Potential. ............................................. 255

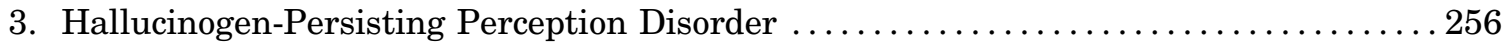

D. Potential Long-Term Physical Side Effects in Uncontrolled Settings ..................256

1. Cardiovascular Side Effects ..................................... 256

X. Recommendations for Future Research ................................. 256

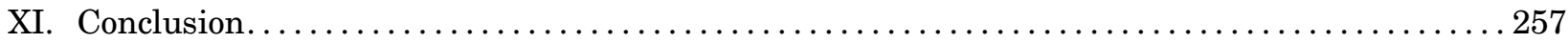

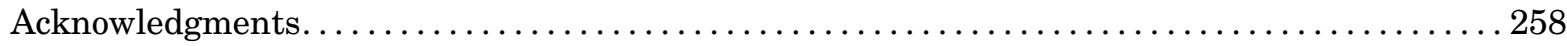

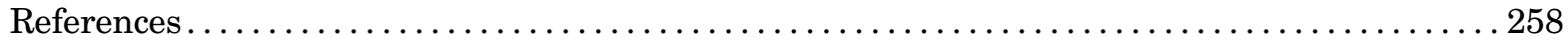

Abstract_-Mounting evidence suggests safety and efficacy of psychedelic compounds as potential novel therapeutics in psychiatry. Ketamine has been approved by the Food and Drug Administration in a new class of antidepressants, and 3,4-methylenedioxymethamphetamine (MDMA) is undergoing phase III clinical trials for post-traumatic stress disorder. Psilocybin and lysergic acid diethylamide (LSD) are being investigated in several phase II and phase I clinical trials. Hence, the concept of psychedelics as therapeutics may be incorporated into modern society. Here, we discuss the main known neurobiological therapeutic mechanisms of psychedelics, which are thought to be mediated by the effects of these compounds on the serotonergic (via $5-H^{-} T_{2 A}$ and $5-H T_{1 A}$ receptors) and glutamatergic [via $N$-methyl-D-aspartate (NMDA) and $\alpha$-amino-3-hydroxy-5-methyl-4-isoxazolepropionic acid (AMPA) receptors] systems. We focus on 1) neuroplasticity mediated by the modulation of mammalian target of rapamycin-, brain-derived neurotrophic factor-, and early growth response-related pathways; 2) immunomodulation via effects on the hypothalamic-pituitaryadrenal axis, nuclear factor $\mathrm{kB}$, and cytokines such as tumor necrosis factor- $\alpha$ and interleukin 1,6 , and 10 
production and release; and 3) modulation of serotonergic, dopaminergic, glutamatergic, GABAergic, and norepinephrinergic receptors, transporters, and turnover systems. We discuss arising concerns and ways to assess potential neurobiological changes, dependence, and immunosuppression. Although larger cohorts are required to corroborate preliminary findings, the results obtained so far are promising and represent a critical opportunity for improvement of pharmacotherapies in psychiatry, an area that has seen limited therapeutic advancement in the last 20 years. Studies are underway that are trying to decouple the psychedelic effects from the therapeutic effects of these compounds.
Significance Statement_-Psychedelic compounds are emerging as potential novel therapeutics in psychiatry. However, understanding of molecular mechanisms mediating improvement remains limited. This paper reviews the available evidence concerning the effects of psychedelic compounds on pathways that modulate neuroplasticity, immunity, and neurotransmitter systems. This work aims to be a reference for psychiatrists who may soon be faced with the possibility of prescribing psychedelic compounds as medications, helping them assess which compound(s) and regimen could be most useful for decreasing specific psychiatric symptoms.

\section{Introduction}

\section{A. Review Outline}

In the last 30 years, the revamped interest in the application of psychedelic compounds in psychiatry has generated a huge body of work on the pharmacological and therapeutic action of these molecules. This has recently led to approval by the FDA of ketamine as a first in a new class of antidepressants (Kim et al., 2019). Other compounds are being tested in multicenter phase III and II clinical trials, such as 3,4-methylenedioxymethamphetamine (MDMA)-augmented psychotherapy for treatment-resistant post-traumatic stress disorder (PTSD) and psilocybin for treatment-resistant major depressive disorder (MDD) (ClinicalTrials.gov). Hence, the clinical implementation of these compounds has begun and may soon expand, indicating that the use of psychedelic compounds for therapeutic purposes may be incorporated into modern society (Rucker et al., 2018; Ona et al., 2019).

Psychiatrists are faced with a new class of therapeutic tools that may be licensed as medicines and can almost immediately improve psychiatric symptoms, but that can elicit profound changes in consciousness and perception. The experiences elicited have been described by participants in clinical trials as among some of the most transformative in their lives. Although this aspect will not be discussed in detail in this review, it raises the critical concept that adequate preparation should be provided to psychiatrists who will prescribe and administer these compounds in clinical settings. Importantly, the context in which the therapy takes place can influence the final outcomes, adding a layer of complexity (Carhart-Harris et al., 2018b). Specialized therapist training is already being provided by research centers currently researching psychedelics and may need to be scaled up should this type of treatment become more widely used (Phelps, 2017; Nutt and Carhart-Harris, 2020).

To elicit significant improvements in the psychiatric symptomatology, in most cases, this type of therapy requires administration of the compound only once or twice over a few weeks, which are preceded and followed by preparation and integration sessions with a trained therapist. A four-step treatment model is becoming standardized in randomized controlled trials (RCTs) and could be applied to the clinic. This model includes 1) the assessment of the patient's mental and physical suitability for this type of therapy; 2) the preparation provided by a trained therapist to the multifaceted possibilities of the "psychological journey," as well as indications on how to navigate potentially challenging experiences and get the most from the therapy; 3 ) the experience session itself, which involves the administration of the compound in comfortable settings, such as a lounge-like environment, the possibility of listening to music and wearing eyeshades, and the continuous presence of a trained therapist, which is available should the patient feel the need to interact verbally or physically; and 4) the integration session to discuss with a trained therapist how the experience might relate to the patient's illness and to help the patient integrate the experience with his or her life. Ideally, further psychotherapeutic integration sessions should be available to process issues or insights that might arise and to provide guidance on how to cultivate lifestyle and cognitive adjustments (Nutt and Carhart-Harris, 2020). The integration part seems especially relevant given that antidepressant responses are enhanced by an enriched environment but can be counteracted by a stressful one (Alboni et al., 2016). Therefore, considering that patients often report significant changes in their value sets, world views, and meaning (Hartogsohn, 2018), which might clash with their preexisting lifestyle generating substantial distress, it is essential that patients are adequately followed up to optimize treatment outcome and avoid undesirable side effects or relapsing into unhealthy habits/patterns (Richards, 2016; Sloshower, 2018; Watts and Luoma, 2020).

Most importantly, the clinical implementation of these compounds requires psychiatrists to be familiarized with the pharmacology of these compounds, the neurobiological mechanisms at the root of therapeutic improvement, and potential mechanisms and pharmacological interactions that could mediate the insurgence 
of more or less severe side effects. Here, we discuss the current understanding of the neurobiology of psychedelic compounds, focusing on compounds which have been, or could soon be, classified as novel psychiatric medications. We discuss the current understanding of the pharmacology underlying the effects of psychedelics on neuroplasticity, immunomodulation, and neurotransmission. When possible, we will draw parallels in terms of current psychiatric pharmacotherapies and how psychedelic compounds weigh up in comparison. The many published results available in the scientific literature show that notable efforts are being poured in this emerging field, and as a result, our understanding of the neurobiology of psychiatric drugs is deepening exponentially. To narrow the scope of this review, we decided to focus on compounds that are already approved as medications or are under scrutiny for their potential application in psychiatry. Other psychedelics may be touched upon if relevant for this review but will not be discussed in depth.

\section{B. Psychiatric Disorders and the Need for Novel Pharmacotherapies}

Psychiatric disorders are a major public health concern and a leading cause of economic burden worldwide, affecting about 350 million people (Wittchen et al., 2011; Whiteford et al., 2013; Global Burden of Disease Study 2013 Collaborators, 2015; Vigo et al., 2019). It is estimated that one in two individuals $(50 \%$ of the population) in high-income countries will meet the diagnosis for at least one psychiatric disorder in his or her lifetime (Wittchen et al., 2011; Kessler et al., 2012). Although this represents a historical peak, in terms of monetary expenditure and years lived with disability, the incidence of these conditions is still dramatically increasing (Vos et al., 2012; Global Burden of Disease Study 2013 Collaborators, 2015). Psychiatric disorders are multifactorial disorders arising from genes $\times$ environment interaction (Wong et al., 2008; Koenen et al., 2009; Flint and Kendler, 2014; Kaufman, 2018). Genetic variabilities among populations (such as clusters of single nucleotide polymorphisms) have been identified that confer vulnerability or resilience to psychiatric disorders, spanning DNA regions involved in neuroplasticity, immune system modulation, and neurotransmission (Caspi et al., 2010; Cross-Disorder Group of the Psychiatric Genomics Consortium, 2013; Smoller, 2016; Wray et al., 2018).

Individuals diagnosed with a psychiatric disorder, which may be chronic or relapsing, have increased odds to develop comorbid systemic illnesses, which aggravate the psychiatric symptomatology in a feedback loop (Koenen et al., 2009; Rogers et al., 2016; Inserra et al., 2018; Sporinova et al., 2019). A further emerging biological contributor to the development of psychiatric disorders is epigenetics, the ensemble of functional chromatin modifications shaped by one's life (Nestler et al., 2016; Kular and Kular, 2018). Contrasting a longstanding paradigm, epigenetic modifications are transgenerational (can be passed onto the offspring) and may well play a role in the current psychiatric epidemics (Tsankova et al., 2007; Yeshurun and Hannan, 2019). Despite tremendous efforts to uncover pathophysiological determinants, available treatments are only partly effective and are still far from ideal. These treatments seldom lead to clinical remission and present significant side effects, delayed therapeutic onset, and residual symptoms (Penn and Tracy, 2012; Duman et al., 2016). Therefore, identifying novel therapeutic strategies is of paramount importance to the current public health system, especially for psychiatric diseases in which no fully efficacious treatments are available, such as PTSD or autism spectrum disorder (ASD). Ideally, the next generation of psychiatric medications will have a fast onset of therapeutic action and few, acceptable, side effects (Duman et al., 2016; Harmer et al., 2017).

\section{Psychedelic Compounds as Novel Therapeutics in Psychiatry: Overview and Comparison with Current Available Treatments}

Preliminary evidence suggests that psychedelic compounds may hold potential as therapeutics for psychiatric disorders. The term "psychedelic" derives from the Greek words meaning "mind manifesting," which refers to the ability of these compounds to bring hidden aspects of the subconscious mind into a conscious framework. Psychedelics induce profound changes in consciousness, perception, emotions, and selfawareness. These effects have been described as paradoxical given that, although acute psychotic-like symptoms are experienced, significant mid- to long-term improvements in psychological well-being are usually reported (Vollenweider and Kometer, 2010; CarhartHarris et al., 2016b; De Gregorio et al., 2018). Preliminary evidence of safety, efficacy, and tolerability generated by clinical trials is available for 1) ketamine, psilocybin, and ayahuasca for recurrent and treatmentresistant MDD (Murrough et al., 2013a; Carhart-Harris et al., 2016a; Palhano-Fontes et al., 2019; Fava et al., 2020; Davis et al., 2020); 2) MDMA and LSD for treatment-resistant PTSD (Mithoefer et al., 2018, 2019; Schmid et al., 2020); 3) psilocybin for obsessivecompulsive disorder (OCD) (Leonard and Rapoport, 1987; Moreno et al., 2006), 4) alcohol abuse (Bogenschutz et al., 2015), and 5) smoking cessation (Johnson et al., 2014); 6) ayahuasca for suicidality (Zeifman et al., 2020); and 7) psilocybin and lysergic acid diethylamide (LSD) for anxiety, depression, pain, and distress associated with a life-threatening illness (Gasser et al., 2014; Griffiths et al., 2016).

First-line pharmacological treatments in unipolar depression, anxiety, PTSD, and OCD revolve around selective serotonin reuptake inhibitors (SSRIs) [such as fluoxetine, fluvoxamine, citalopram and paroxetine, 
which inhibit serotonin (5-HT) reuptake at the synapse] and serotonin-norepinephrine reuptake inhibitors (SNRIs) [such as clomipramine, venlafaxine, duloxetine, milnacipran, and levomilnacipran, which inhibit 5HT and norepinephrine (NE) reuptake at the synapse]. Second-line treatments include the second-generation (atypical) antipsychotics acting on $5-\mathrm{HT}_{2 \mathrm{~A}}, 5-\mathrm{HT}_{1 \mathrm{~A}}$, and D2 receptors (Gobbi et al., 2018). In some cases, tricyclic antidepressants (such as imipramine and amitriptyline, which also inhibit monoamine reuptake) are similarly, or more, effective for the treatment of some patients, especially in hospitalized patients with severe depression (Bauer et al., 2002; Bandelow et al., 2008).

Second-generation (or atypical) antipsychotics (such as clozapine, olanzapine, quetiapine, aripiprazole, risperidone) are used for the long-term treatment of psychoses (Kapur and Remington, 1996; Hasan et al., $2012,2013)$ since they have less risk of producing extrapyramidal symptoms as compared with typical antipsychotics. Atypical antipsychotics have a mixed $\mathrm{DA} / 5-\mathrm{HT}_{2 \mathrm{~A} / 1 \mathrm{~A}}$ receptor pharmacological profile, including antagonist action at the level of $5-\mathrm{HT}_{2 \mathrm{~A}}$ receptors (olanzapine and quetiapine) or partial agonism at the level of D2 receptor (aripiprazole). Clozapine, which is a potent $5-\mathrm{HT}_{2 \mathrm{~A} / 2 \mathrm{C}}$ receptor antagonist, is suggested as second-line treatment in these cases because of its superior efficacy in treatment-resistant schizophrenia [for revision of the mechanism of action, see Comai et al. (2012)]. Interestingly for this context, phencyclidine (PCP) and other $5-\mathrm{HT}_{2 \mathrm{~A}}$ receptor agonist hallucinogens at high doses have been proposed as a model of animal psychosis that can be reversed by atypical antipsychotics with $5-\mathrm{HT}_{2 \mathrm{~A}}$ and $\mathrm{D} 2$ receptor antagonism (Geyer and Ellenbroek, 2003).

Pharmacological overlap exists between the serotonergic action of psychedelic compounds, routinely prescribed psychiatric drugs such as SSRIs (about $80 \%$ of all prescribed antidepressants), and atypical (secondgeneration) antipsychotics, mostly stemming from the interaction with one or more 5-HT receptors or with the 5-HT transporter (SERT) (Celada et al., 2004). Psychedelics are agonists or partial agonists at presynaptic 5$\mathrm{HT}_{1 \mathrm{~A}}$ receptors and postsynaptic $5-\mathrm{HT}_{1 \mathrm{~A}}$ and $5-\mathrm{HT}_{2 \mathrm{~A}}$ receptors; the onset of antidepressant effects with other antidepressants seems to require the hippocampal upregulation of presynaptic $5-\mathrm{HT}_{1 \mathrm{~A}}$ receptor-mediated transmission, suggesting a potential common mechanism of therapeutic improvement (Haddjeri et al., 1998). Both the SSRIs clomipramine and LSD increase 5-HT synaptic availability and inhibit dorsal raphe nucleus (DRN)/5-HT cell firing (Freedman, 1961; Aghajanian et al., 1968; Adell and Artigas, 1991; De Gregorio et al., 2016b). Most antidepressants also downregulate $5-\mathrm{HT}_{2 \mathrm{~A}}$ receptor expression, and although evidence for a downregulation of $5-\mathrm{HT}_{2 \mathrm{~A}}$ receptors in response to psychedelics remains limited, such an effect cannot be excluded (Gray and Roth, 2001).
One study found a decrease in 5-HT binding sites in long-term ayahuasca users, suggesting that similarly to currently approved psychiatric drugs, psychedelic compounds affect the homeostasis of the 5-HT system via downregulating $5-\mathrm{HT}_{2 \mathrm{~A}}$ receptor expression (Callaway et al., 1994). Animal studies corroborate this notion, confirming a downregulation of $5-\mathrm{HT}_{2 \mathrm{~A}}$ receptor expression and functional signaling changes in response to the repeated administration of $\mathrm{LSD}$, which is concomitant to the onset of behavioral tolerance (Gresch et al., 2005; Martin et al., 2014). On the one hand, atypical antipsychotics are potent $5-\mathrm{HT}_{2 \mathrm{~A} / 2 \mathrm{C}}$ receptors antagonists (and weak D2 receptor antagonists), and their antipsychotic potency positively correlates with their profile of $5-\mathrm{HT}_{2 \mathrm{~A} / 2 \mathrm{C}}$ and $\mathrm{D} 2$ receptor affinity (Richtand et al., 2007; Lord et al., 2017). This action is thought to counteract the aberrant dopaminergic neurotransmission in SCZ by directly or indirectly increasing $5-\mathrm{HT}_{1 \mathrm{~A}}$ receptor-mediated neurotransmission and modulating the biosynthesis and release of dopamine, ultimately eliciting antipsychotic activity (Schmidt et al., 1995; Kehne et al., 1996; Rollema et al., 1997; Lieberman et al., 1998; Díaz-Mataix et al., 2005; Meltzer and Huang, 2008;; Meltzer and Massey, 2011). On the other hand, most psychedelics are $5-\mathrm{HT}_{2 \mathrm{~A}}$ receptor agonists, and this action is thought to mediate their psychedelic, and hallucinogenic, effects (Kroeze and Roth, 1998; Meltzer, 1999; Celada et al., 2004; Nichols, 2016).

Although the mechanism of action of SSRIs and psychedelics appears somewhat similar-for example, SSRIs and LSD both decrease the 5-HT firing activity through the stimulation of $5-\mathrm{HT}_{1 \mathrm{~A}}$ autoreceptor (De Gregorio, 2016b)—one discrepancy between the dynamics of therapeutic improvement elicited by SSRIs and psychedelics is the timing: psychedelics appear to induce almost immediate improvements in psychiatric symptoms, whereas routinely approved antidepressants require days to weeks (Blier and de Montigny, 1994). This can be explained by the fact that SSRIs elicit noticeable therapeutic improvements after the $5-\mathrm{HT}_{1 \mathrm{~A}}$ autoreceptor is desensitized and the firing of 5-HT is restored, and this requires a significant time frame (days to weeks) to elicit pre- and postsynaptic adjustments that translate to therapeutic improvements, which are thought to be mediated by epigenetic processes (Tsankova et al., 2007; Baudry et al., 2019). Psychedelics, like LSD, also require a desensitization of the $5-\mathrm{HT}_{1 \mathrm{~A}}$ receptor (unpublished data) to elicit anxiolytic effects, but this delay is shorter, and it is therefore possible that the therapeutic improvements are elicited through mechanisms other than $5-\mathrm{HT}_{1 \mathrm{~A}}$ receptor desensitization, including direct action on cortical $5-\mathrm{HT}_{2 \mathrm{~A}}$ and $\alpha$-amino-3-hydroxy-5-methyl-4-isoxazole propionic acid (AMPA) receptors (Marek and Aghajanian, 1996). Although further studies are required to answer this research question, none of the proposed possibilities can be categorically excluded at this stage. 
The studies performed so far that investigate the effects of psychedelic compounds for a variety of psychiatric illnesses have yielded encouraging results, suggesting that these compounds might have a place in the treatment of several psychiatric disorders that represent a huge economic burden over the health system and society as a whole. However, one major barrier hampers preclinical and clinical research on these compounds, ultimately hindering the potential application of these substances in psychiatry and medicine in general: their classification as schedule 1 substances in the United States and their homologous classifications in other countries. According to their status, these compounds are drugs with high abuse potential and no medical application. Although such classification is necessary for substances that objectively display highly addictive or highly reward-stimulating profiles, the classification of these compounds as schedule 1 appears not to entirely reflect their pharmacological profiles, including low addictive liability (discussed throughout this review).

Steps that might reconcile this duality include but are not limited to 1) increased preclinical and phase I to III clinical trials demonstrating the clinical efficacy of this class of compounds for specific mental diseases and approval by drug regulatory governmental agencies (i.e., FDA, European Medicines Agency, Health Canada); 2) an evidence-based approach by legislators and funding bodies; 3 ) destigmatization of these compounds by society, which requires efforts from regulatory government and funding bodies, academic institutions, the health system, and mass media; and 4) priority funding dedicated to answering such questions through evidence-based scientific investigations. Given that no medicine is devoid of risks, such steps could help define more clearly the boundaries between the real risks versus the real benefits brought on by the application of these compounds in psychiatry and in medicine more generally speaking. Ultimately, if the results obtained will be positive, such an evidence-based approach could lead to the use of these compounds in medical practice. However, this requires an evidence-based debate to catalyze the acknowledgment of their therapeutic utility by regulatory bodies.

\section{Classical or Serotonergic Psychedelics versus Nonclassical Psychedelics: Definition}

The definition of psychedelics is still based on a classification from the $1960 \mathrm{~s}$, and it continues to be a matter of debate. Classic or serotoninergic psychedelic compounds are so called mainly because they interact with the serotonergic system and most of them derive from plants or are semisynthetics (Andén et al., 1968; Glennon et al., 1984; Vollenweider et al., 1998). Serotonergic psychedelics include 1) the semisynthetic ergoline LSD; 2) plant-derived tryptamines, such as psilocybin (the active ingredient found in "magic mushrooms"),
$N, N$-dimethyltryptamine (DMT, the active ingredient in ayahuasca), 5-methoxy- $N, N$-dimethyltryptamine (5$\mathrm{MeO}-\mathrm{DMT}$ ), and lysergic acid amide (the active ingredient in morning glories); and 3) phenethylamines, such as mescaline (the active ingredient in the peyote and San Pedro cacti), and phenethylamine-based synthetic designer drugs (Vollenweider et al., 2007; Maciulaitis et al., 2008; Passie et al., 2008; Vollenweider and Kometer, 2010; Dean et al., 2013; Steiner and Leistner, 2018; Dinis-Oliveira et al., 2019). In some cases, they share part of the chemical structure with the endogenous neurotransmitter serotonin $(5-\mathrm{HT})$-in particular, the indole scaffold. However, some of them, like mescaline, do not possess an indole but are still considered serotonergic psychedelics.

Serotonergic psychedelics are mostly found in nature (e.g., psilocybin, DMT, mescaline, and LSD, which is derived from naturally occurring ergotamine); however, LSD can also be created in a laboratory by modifying natural psychedelics (Hofmann, 1979). Newer analogs and derivatives of pharmacophore lysergamides, tryptamine, and phenethylamine structures are serotoninergic psychedelics even if considered "nonclassical" (Johnson et al., 2019). Despite their name, however, the pharmacology of serotonergic classic psychedelics is not limited to 5-HTRs but has several layers of complexity that involve several receptor families, homoand heteroreceptor complexes, and biased intracellular cascades. The complete pharmacological profile of these compounds should therefore be considered when trying to dissect the molecular mechanisms underlying psychedelic-induced therapeutic improvements.

LSD is a semisynthetic ergosterol that can be derived by the naturally occurring ergot alkaloid lysergic acid, which is contained in the rye parasite Claviceps purpurea (Castagnoli and Mantle, 1966; Tsai et al., 1995; Tudzynski and Scheffer, 2004). Psilocybin (4-phosphoryloxy- $N, N$-dimethyltryptamine) is a substituted indolealkylamine (Hofmann et al., 1958). Ayahuasca is an ethnobotanical preparation used in the Amazon Basin for religious, spiritual, and healing purposes (McKenna and Towers, 1985; Frecska et al., 2016). The tea, which is brewed from plants indigenous to the rainforest, contains DMT and $\beta$-carboline alkaloids (harmine, harmaline, and tetrahydroharmine) (McKenna et al., 1984; Yritia et al., 2002; Pires et al., 2009). The $\beta$-carbolines harmol, harmine, harmaline, and tetrahydroharmine are obtained from Banisteriopsis caapi (ayahuasca, or "vine of the souls") and function as monoamine oxidase inhibitors (MAOIs) to block the metabolism of DMT, rendering it orally active (Pähkla et al., 2000; Yritia et al., 2002; Gambelunghe et al., 2008; Carbonaro et al., 2015). These compounds are tricyclic indole alkaloids, which resemble tryptamines (Hamill et al., 2019). The presence of an endogenous counterpart in the pineal gland and retina, 6-methoxytetrahydro- $\beta$-carbolin (pinoline), has been reported 
(Langer et al., 1984; Leino, 1984). A close relative of DMT, 5-MeO-DMT, is found in several plants (such as Anadenanthera peregrine) and in certain species of toads (such as Incilius alvarius, which also produces bufotenine) (Glennon and Rosecrans, 1982; Shen et al., 2010).

\section{E. Dissociative Anesthetics}

Compounds that trigger psychedelic-like effects and a dissociative-like state, which partly resembles the anesthetic state, are termed dissociative anesthetics (Evoniuk et al., 1991; Yamakura et al., 2000; Zorumski et al., 2016). Dissociation refers to the disruption of usually integrated functions, such as consciousness, memory, emotions, and behavior (Carlson et al., 2018). Renewed interest has been placed on this class of compounds following the FDA approval of esketamine as a novel antidepressant (Kim et al., 2019; Krystal et al., 2019). The other most known, and more infamous, dissociative anesthetic is PCP ("angel dust"), from which ketamine was derived (Budd and Lindstrom, 1982; Bertron et al., 2018).

Ketamine is a phenylcyclohexylamine derivative consisting of two optical enantiomers, $(S)$-ketamine and $(R)$-ketamine (Adams et al., 1978, 1981; White et al., 1985; Leung and Baillie, 1989; Oye et al., 1992; Berman et al., 2000; Yeung et al., 2010; Hashimoto, 2014). The racemic mixture esketamine [containing the $(S)$ and $(R)$ isomers in equal parts], has been approved as a medication by the FDA for treatment-resistant MDD (Zhou and Zhao, 2000; Cristea and Naudet, 2019). Ketamine also possesses analgesic activity, which might stem from interactions with the serotonergic, opioidergic, and endocannabinoid systems, and several voltage-gated sodium channels (Zhou and Zhao, 2000; Schnoebel et al., 2005; Ferreira et al., 2018; Savic Vujovic et al., 2019).

\section{F. Empathogens-Entactogens}

A third class of compounds structurally related to psychedelic phenylethylamines has been termed empathogen-entactogen ("having the connotation of producing a touching within") (Nichols, 1986; Vollenweider, 2001), which we will refer to as entactogens for the rest of this review. Compounds in this class include MDMA and 3,4-methylenedioxyamphetamine (MDA) (Riedlinger and Riedlinger, 1994). Although entactogens do not induce a full-blown distortion of perception and consciousness, as with the other psychedelics discussed here, their empathogenic properties are nonetheless relevant for this review. These effects have been broadly examined in psychotherapy augmentation in the context of PTSD, and potential effects on fear extinction and memory reconsolidation have been suggested (Feduccia and Mithoefer, 2018; Sessa et al., 2019; Bahji et al., 2020). MDMA has been considered by the FDA superior to current pharmacological therapies for
PTSD and granted the breakthrough designation for treatment-resistant PTSD (Feduccia et al., 2019).

\section{G. Full Dosing versus Microdosing}

Most human studies so far have used "full psychedelic doses," which elicit profoundly altered states of consciousness. The occurrence of such effects is perhaps part of the reason why psychedelic compounds are classified as schedule 1 drugs-that is, substances with high abuse potential and no recognized medical value. More recently, somewhat circumnavigating the hurdle of deeply altered states of consciousness, the concept of "microdosing" has emerged, which consists in taking a "dose of drug that is $1 \%$ of the pharmacologically active dose, up to a maximum of $100 \mu \mathrm{g}$ " (Tewari and Mukherjee, 2010). This kind of dosage is well below the "psychedelic threshold," eliciting minimal or no psychoactive effects, and it is mostly sought for cognitive and emotional enhancement (Hutten et al., 2019; Rifkin et al., 2020) or by patients who are dissatisfied with conventional treatments to improve mental health or cease prescription or illegal substance use (Hutten et al., 2019; Lea et al., 2020). Despite the lack of systematic studies investigating beneficial outcomes and potential long-term side effects of microdosing, a growing number of individuals are seeking this alternative therapeutic approach, sometimes believing it might be a universal remedy for all somatic and psychological ailments. However, the lack of supporting clinical research has prompted some researchers in the field to demystify these beliefs, highlighting that the notion that microdosing enhances cognition and "cures" psychiatric illness is not supported by scientific research in the field, which in fact has more questions than answers (Kuypers et al., 2019; Preller, 2019).

The most used substances for microdosing among the general population are LSD, followed by psilocybin (Rosenbaum et al., 2020). Microdosing has been described by microdosers as "embracing traditional middle-class values" (Webb et al., 2019), and this approach might be more easily managed by naïve patients compared with full doses and better received by psychiatrists who have no experience in dealing with psychedelic effects. It may even become a therapy for the elderly, and preliminary evidence suggests safety and tolerability of LSD microdosing in elderly populations (Family et al., 2020). Preliminary reports suggest that microdosing has low but appreciable consciousness-altering effects (Prochazkova et al., 2018; Bershad et al., 2019; Yanakieva et al., 2019; Hutten et al., 2020) and enhances mood and focus (Anderson et al., 2019; Webb et al., 2019; Lea et al., 2020; Hutten et al., 2020), cognitive performance (Prochazkova et al., 2018), and empathy (Johnstad, 2018) and decreases mind wandering (Polito and Stevenson, 2019). Microdosing might also help alleviate symptoms of existing medical conditions (Fadiman and Korb, 2019) potentially given the 
powerful anti-inflammatory effects of psychedelic 5$\mathrm{HT}_{2 \mathrm{~A}}$ receptor agonists (Yu et al., 2008; Nau et al., 2013, 2015; Flanagan et al., 2019b). In fact, strong biological effects have been reported in response to very low doses of psychedelic compounds, such as the potent anti-inflammatory effects of the highly selective $5-\mathrm{HT}_{2 \mathrm{~A}}$ receptor agonist 2,5-dimethoxy-4-iodoamphetamine (DOI), demonstrated extensively in both in vivo and in vitro paradigms (Nau et al., 2013, 2015; Flanagan and Nichols, 2018; Flanagan et al., 2019a,b). Although further studies are required, preliminary findings suggest that microdoses might elicit therapeutic improvements without eliciting profound changes in consciousness. Some have reported microdosing in preparation for full doses of other psychedelics (Johnstad, 2018). Other potential applications are the treatment of migraines, cluster headaches (Andersson et al., 2017), and pain conditions (Johnstad, 2018). Concerning LSD, some authors have suggested that doses $<20 \mu \mathrm{g}$ might be considered microdoses, doses between 21 and $30 \mu \mathrm{g}$ might be considered minidoses, and doses $>30$ might be considered psychedelic doses (Holze et al., 2020). In preclinical studies, antidepressant-like effects and enhancement of fear-memory extinction were observed (Cameron et al., 2019), suggesting microdosing might be valuable for comorbid depression and PTSD.

Contrasting the increasingly popular belief that microdosing might represent a universal cure, preliminary preclinical findings support the notion that microdosing might have anxiogenic (not anxiolytic) and neurotic effects (Horsley et al., 2018; Anderson et al., 2019; Polito and Stevenson, 2019), induce confusion (Hutten et al., 2020) and potentially impact metabolism and hinder synaptic plasticity (Cameron et al., 2019), which unequivocally are undesirable effects in psychiatry. Although the concept of microdosing raises the intriguing possibility of decoupling the therapeutic benefits of psychedelic compounds from their psychedelic effects, whether this type of regimen 1) elicits the full spectrum of therapeutic improvement as full doses, 2) presents abuse potential, and 3) elicits acute and long-term side effects remains to be ascertained (the available evidence is discussed throughout the present work).

Preliminary data suggesting that about 1 in 5 patients experience physical discomfort and that about 1 in 10 experience anxiety when microdosing (Anderson et al., 2019) should be considered in future clinical trials, and studies should aim at elucidating the underlying mechanisms, attempting to pharmacologically counteract these side effects. The main off-target effects that are cause for concern so far revolve around the repeated stimulation of cardiac $5-\mathrm{HT}_{2 \mathrm{~B}}$ receptor by microdosing serotonergic psychedelics, which might increase the likelihood of developing cardiovascular disease (discussed in the Abuse Potential and Potential Long-Term Side Effects of Psychedelic Compounds and Side Effects of Psychedelic Compounds sections). Other drugs with $5-\mathrm{HT}_{2 \mathrm{~B}}$ receptor activity have been retracted from the market because of these effects (Connolly et al., 1997), prompting the need to assess potential deleterious effects of psychedelic microdosing on repeated cardiac $5-\mathrm{HT}_{2 \mathrm{~B}}$ receptor stimulation.

Although we do not discredit the possibility that microdosing may potentially be a valuable therapeutic approach, there is not enough available evidence to support this possibility yet. Further larger RCTs are needed to determine the safety, efficacy, and tolerability of microdosing on large cohorts while systematically assessing potential acute and long-term side effects that might arise from this type of treatment. Undoubtedly, given the promising results obtained so far, the increasing number of individuals who self-medicate with psychedelics, and the safety concerns revolving around potential neurobiological and systemic side effects, priority governmental funding should be made available to fill this knowledge gap. Ongoing and future studies should aim at identifying the specific indication, doses, and duration, as well as short- and long-term side effects of each psychedelic compound for specific psychiatric or somatic conditions.

\section{H. Historical Studies}

Aside from pharmacological and behavioral studies in rodents (Uyeno and Benson, 1965; Bílková et al., 1971a,b), cats (Hobson, 1964; Kay and Martin, 1978), spiders, (Groh and Lemieux, 1968), snails (Abramson and Jarvik, 1955), and elephants (West et al., 1962; Siegel, 1984), hundreds of human studies involving tens of thousands of people were performed with psychedelic compounds between the 1950s and 1970s. The main areas of interest included 1) psychotherapy augmentation (Cohen and Eisner, 1959; Pahnke et al., 1970), 2) schizophrenia research (Osmond and Smythies, 1952; Cholden et al., 1955; Bolton, 1962), 3) trauma retrieval (Spencer, 1963), 4) social impairments (Cheek and Holstein, 1971; Krsiak et al., 1971; Vojtĕchovský et al., 1972), 5) alcoholism (O'Reilly and Funk, 1964; Smart and Storm, 1964), 6) neurosis (Denson and Sydiaha, 1970), and 7) cognitive enhancement (Jarvik et al., 1955; Goldberger, 1966). Case reports are available in which LSD treatment was reported to improve sexual perversion and pedophilic impulses (Ball and Armstrong, 1961) and ameliorate anxiety-induced compulsive habits, as well as to "cure" nonbinary sexuality and "relieve the fear of being homosexual" (Abramson, 1955; Ball and Armstrong, 1961). Although most of these studies do not satisfy contemporary scientific research standards, they nonetheless represent a wealth of valuable investigations and could help with the development of modern study design.

\section{Ongoing Clinical Trials}

Contemporary RCTs investigating safety and efficacy of psychedelics for the amelioration of mood disorders 
suggest that these compounds produce a number of desirable improvements in psychiatric populations and healthy volunteers, such as 1) rapid and sustained antidepressant and anxiolytic effects (Murrough et al., 2013b; Carhart-Harris et al., 2016a, 2018; PalhanoFontes et al., 2019), 2) decreased suicidality and emotional distress (Hendricks et al., 2015; Argento et al., 2017), 3) positive personality changes (Bouso et al., 2018), 4) brain activity and connectivity changes (Carhart-Harris et al., 2012; Palhano-Fontes et al., 2015), and 5) long-term neuromorphologic changes, which correlate with positive psychological outcomes (Bouso et al., 2015).

After many years of preclinical and clinical research, esketamine has been approved by the FDA as a medication for treatment-resistant depression. As of August 2020 , there are 260 clinical trials investigating ketamine for the treatment of several disease states that are listed as ongoing, recruiting, or not yet recruiting on ClinicalTrials.gov (for a list of clinical trials investigating ketamine application in psychiatry, see Supplemental Table 1) [also reviewed in Peyrovian et al. (2020))]. Ongoing and future clinical trials aim to assess the effects of ketamine on 1) suicidality; 2) treatmentresistant MDD; 3) bipolar disorder (BD); 4) ASD; 5) borderline personality disorder; 6) cluster headaches; 7) epilepsy; 8) asthma; 9) subarachnoid hemorrhage; 10) renal colic; 11) opioid, alcohol, cocaine, marijuana, and cigarette use; and 12) as a pre- or perioperative preventative antidepressant in populations with depression. Nevertheless, several studies are investigating the analgesic properties of ketamine for critically ill patients with cancer, pediatric fracture reduction, traumatic injuries, and major surgeries. Other RCTs involving ketamine are investigating neurobiological and biochemical biomarkers of successful ketamine antidepressant treatment, which may be used as treatment-efficacy predictors in treatment-resistant populations (reviewed in Supplemental Table 1).

The focus of MDMA clinical research has mostly revolved around the empathogenic effects of this substance, which seems useful as an augmenting agent in psychotherapy (Mithoefer et al., 2016). In controlled clinical settings, MDMA induces a relaxed euphoric state, feelings of emotional openness, enhanced empathy, and disinhibition, creating a cathartic moment in which trauma can be reaccessed, reelaborated, and integrated (Liechti et al., 2001; Holze et al., 2020). Although phase III RCTs are investigating the safety of large-scale employment of MDMAaugmented psychotherapy for PTSD survivors, other trials are investigating the safety and efficacy of the drug for the treatment of ASD and alcohol abuse disorder and the brain circuits mediating the observed clinical improvements (see Table 1 for a list of clinical trials investigating the application of MDMA in psychiatry).
Investigations into the potential therapeutic effects of psilocybin have yielded promising results for treatmentresistant depression (Carhart-Harris et al., 2016a, 2017, 2018), tobacco addiction (Garcia-Romeu et al., 2014; Johnson et al., 2014), and OCD (Moreno et al., 2006), as well as anxiety and distress associated with a life-threatening illness (Griffiths et al., 2016; Ross et al., 2016). Ongoing RCTs are investigating the utility of psilocybin for MDD, treatment-resistant MDD, alcohol use disorder, smoking cessation, cocaine addiction, anorexia nervosa, cognitive impairment and Alzheimer disease, OCD, chronic cluster headache, migraine headache, and post-traumatic headache (see Table 2 for a list of clinical trials investigating the application of psilocybin in psychiatry).

LSD has been investigated in RCTs for its anxiolytic properties (Gasser et al., 2014; Gasser et al., 2015). LSD seems to help patients with a potentially lifethreatening illness elaborate and accept the emotions connected to the potentially terminal nature of their illness. The main improvements observed include decreased anxiety and depression, increased acceptance, and decreased fear toward their potential imminent death (Gasser et al., 2015). Ongoing RCTs are assessing the efficacy of LSD for the treatment of MDD and anxiety disorders and cluster headache. Studies on LSD microdosing are also underway (see Table 3 for a list of clinical trials investigating the application of LSD in psychiatry).

Although ayahuasca has been shown to rapidly relieve depression symptoms in treatment-refractory depression in controlled clinical settings (Osório et al., 2015; Palhano-Fontes et al., 2019), the use of DMT and $5-\mathrm{MeO}-\mathrm{DMT}$ in naturalistic settings is receiving considerable attention given the rapid improvements elicited in several mental health domains accompanied by longlasting decreases in drug and alcohol use and abuse (Davis et al., 2019; Uthaug et al., 2019, 2020; GarciaRomeu et al., 2020). In vitro evidence supports the notion that 5-MeO-DMT might be beneficial for drug and alcohol addiction. For example, 5-MeO-DMT downregulates metabotropic glutamate receptor (mGluR) 5 in human cerebral organoids, and this gene is involved in alcohol- and drug-induced rewards (Dakic et al., 2017) (see Table 4 for a list of clinical trials investigating the application of ayahuasca and DMT in psychiatry).

\section{Psychedelic Compounds as Neuroplastic Agents}

\section{A. Neuroplasticity Impairments in Psychiatric Disorders}

Neuroplasticity refers to the ability of the nervous system to respond to internal and external stimuli via a remodulation of its physical structure and functional connections (Pittenger and Duman, 2008; Cramer et al., 2011). Neuroplasticity occurs on several levels, starting 

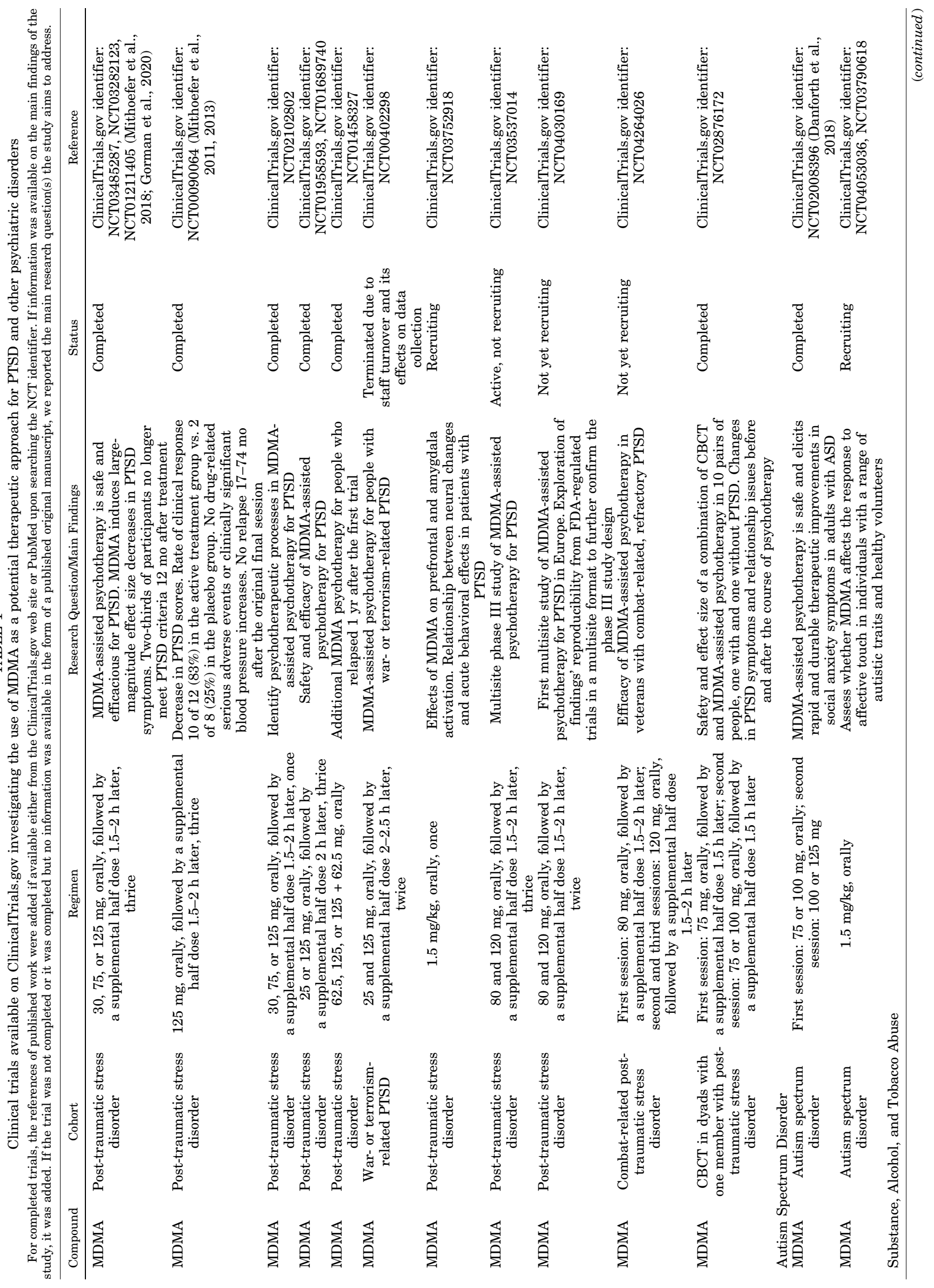


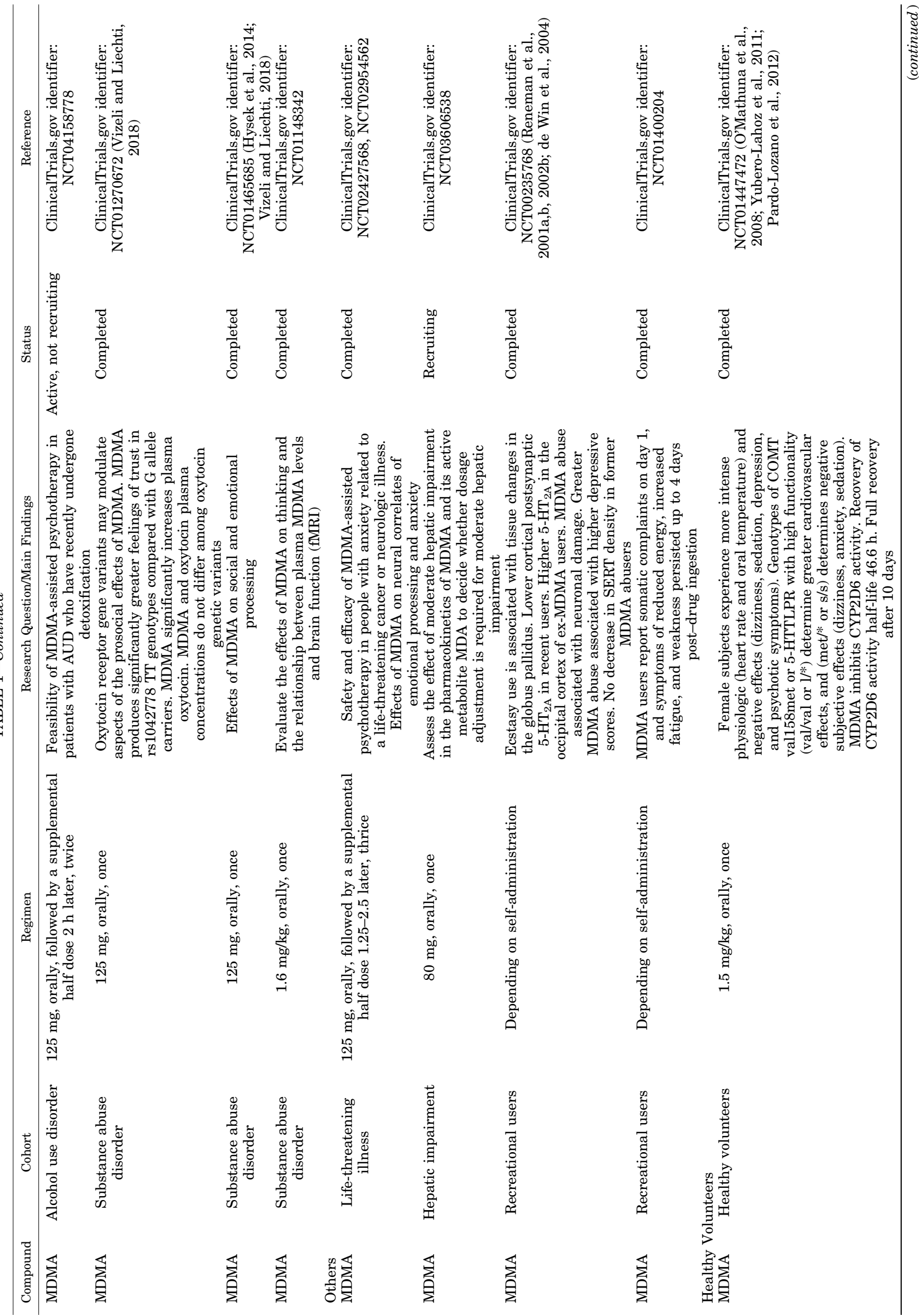




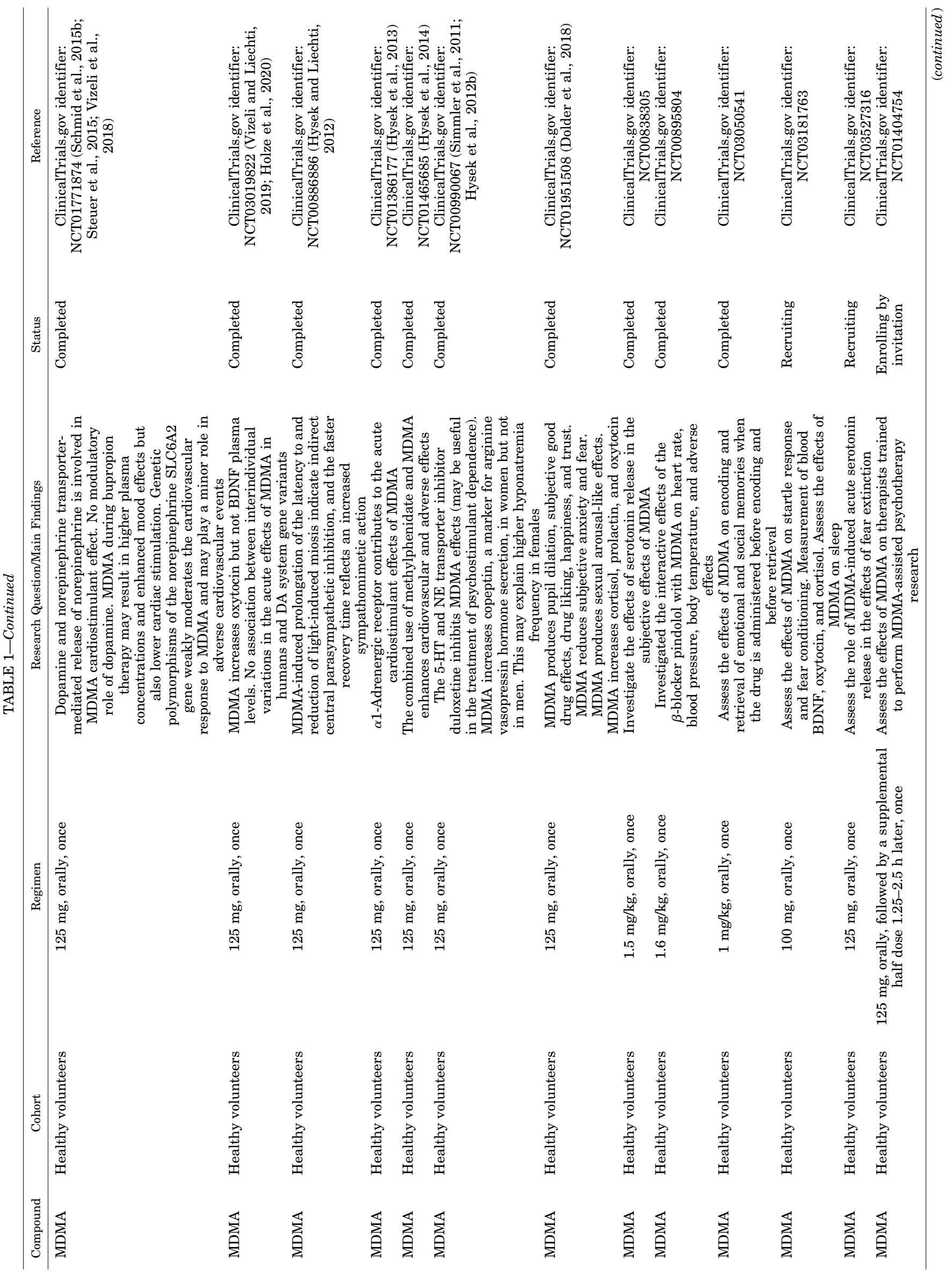


from signal transduction, to gene expression, to synaptic, neuronal, network, and whole-brain adaptations (Duman, 2002; Pittenger and Duman, 2008; Abel et al., 2013). Ultimately, if neuroplasticity leads to a gain of function, it is useful for adaptation, shaping behaviors which help the system adapt to a changing environment (Cramer et al., 2011). However, a dysregulation of neuroplasticity can lead to detrimental adaptive changes and the development of psychiatric illness (Kuipers et al., 2003). Genetic variability in DNA regions that span areas involved in neuroplasticity represent resilience or vulnerability factors in developing psychiatric disorders (Belsky et al., 2009). Not surprisingly, psychiatric drugs elicit therapeutic improvements on behavior at least partially via a remodulation of neuroplasticity-related processes, events which ultimately tend to restore neuroplasticity and behavior (Duman et al., 1994; D'Sa and Duman, 2002).

Psychiatric disorders share neuroplasticity impairments, such as the reduction in hippocampal (MacQueen et al., 2003) and cortical volumes (Bremner et al., 2000; Schmaal et al., 2017) observed in patients with depression and the progressive prefrontal atrophy observed in patients with PTSD who had worsening symptoms (Cardenas et al., 2011). Although the trend appears to be an overall reduction in brain volume in psychiatric disorders, condition-specific symptoms are associated with discrete neuromorphologic changes. For example, greater self-reported anxiety correlates with greater inferior temporal cortex volume decrease in PTSD, contrasting with the slight global volume increase in patients with MDD, or higher scores of selfreported depression, which are correlated with a slight overall volumetric decrease in PTSD together with a slight increase in cuneus and precuneus volume (Kroes et al., 2011). The expression of neurotrophic factors, such as brain-derived neurotrophic factor (BDNF), which modulates neurogenesis, is decreased in psychiatric disorders (Malberg et al., 2000; Molendijk et al., 2014).

Neurogenesis is an essential step to achieve antidepressant outcomes in animal models and humans, and its blockade leads to the development of depressive-like behavior, making it a critical mediator of stress resilience and susceptibility (Ampuero et al., 2010; Kraus et al., 2017). Accordingly, postmortem studies found a reduction in hippocampal dentate gyrus granule cells in medication-free patients with depression compared with treated patients and controls (Gururajan et al., 2016). Neurogenesis-enhancing approaches also seem useful for PTSD paradigms by boosting hippocampal neurogenesis and promoting fear-memory extinction, ultimately decreasing PTSD-like behavior (Ishikawa et al., 2016, 2019). Similar to psychiatric disorders, neurodegenerative disorders are accompanied by decreased neurogenesis (Hollands et al., 2017), although high comorbidity exists among psychiatric and neurologic conditions (Silberstein, 2001; Nuti et al., 2004; 


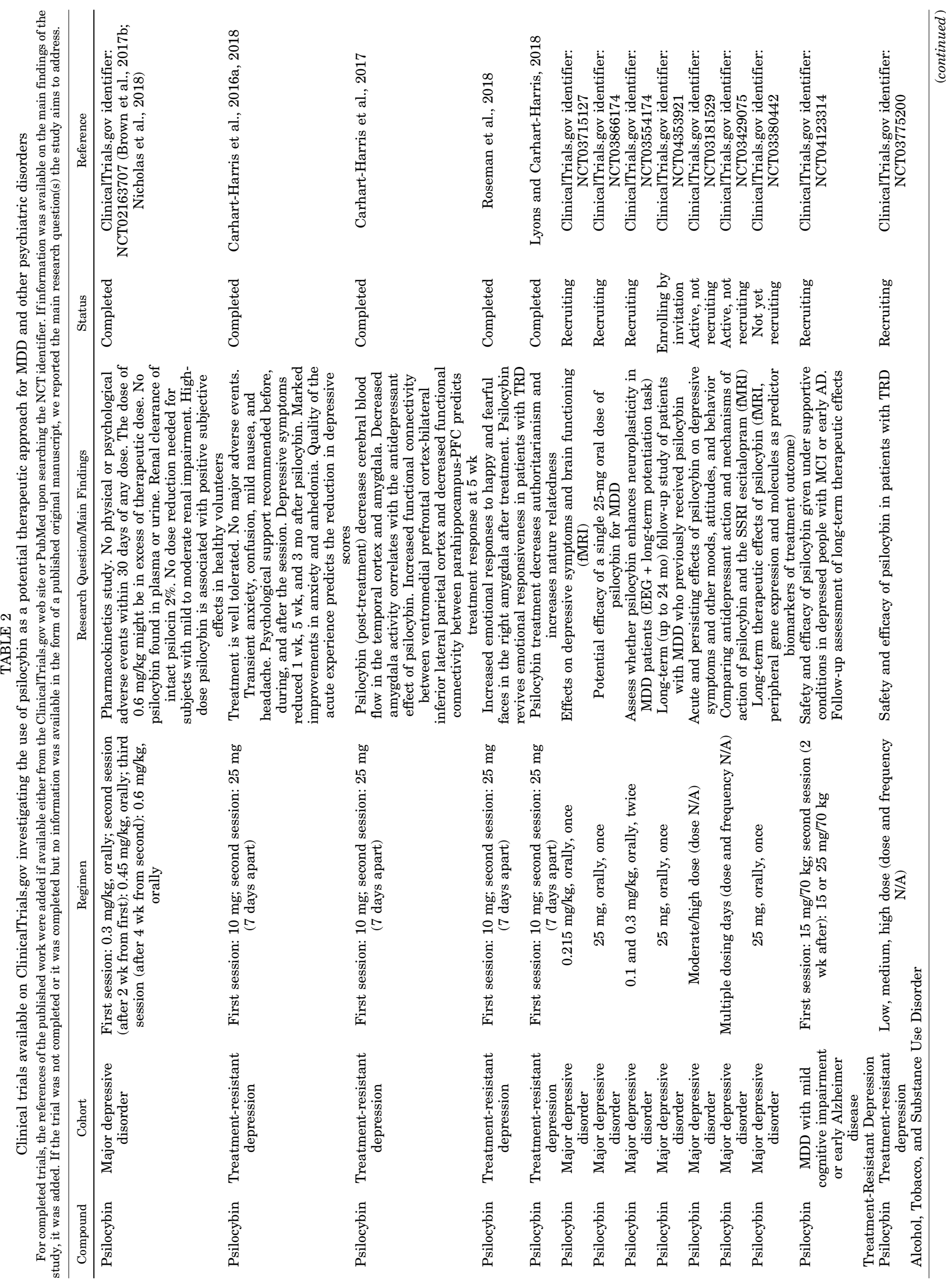




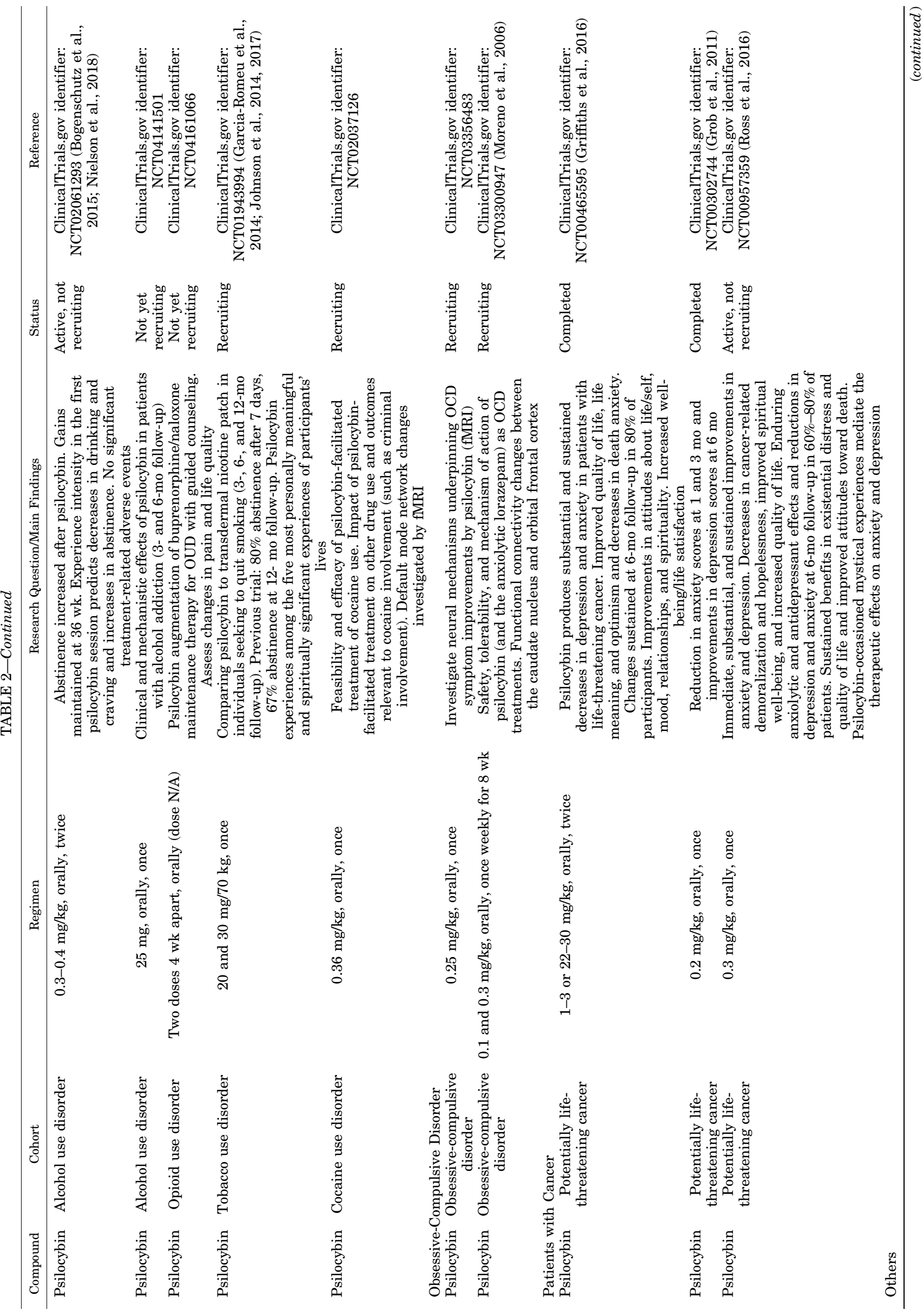




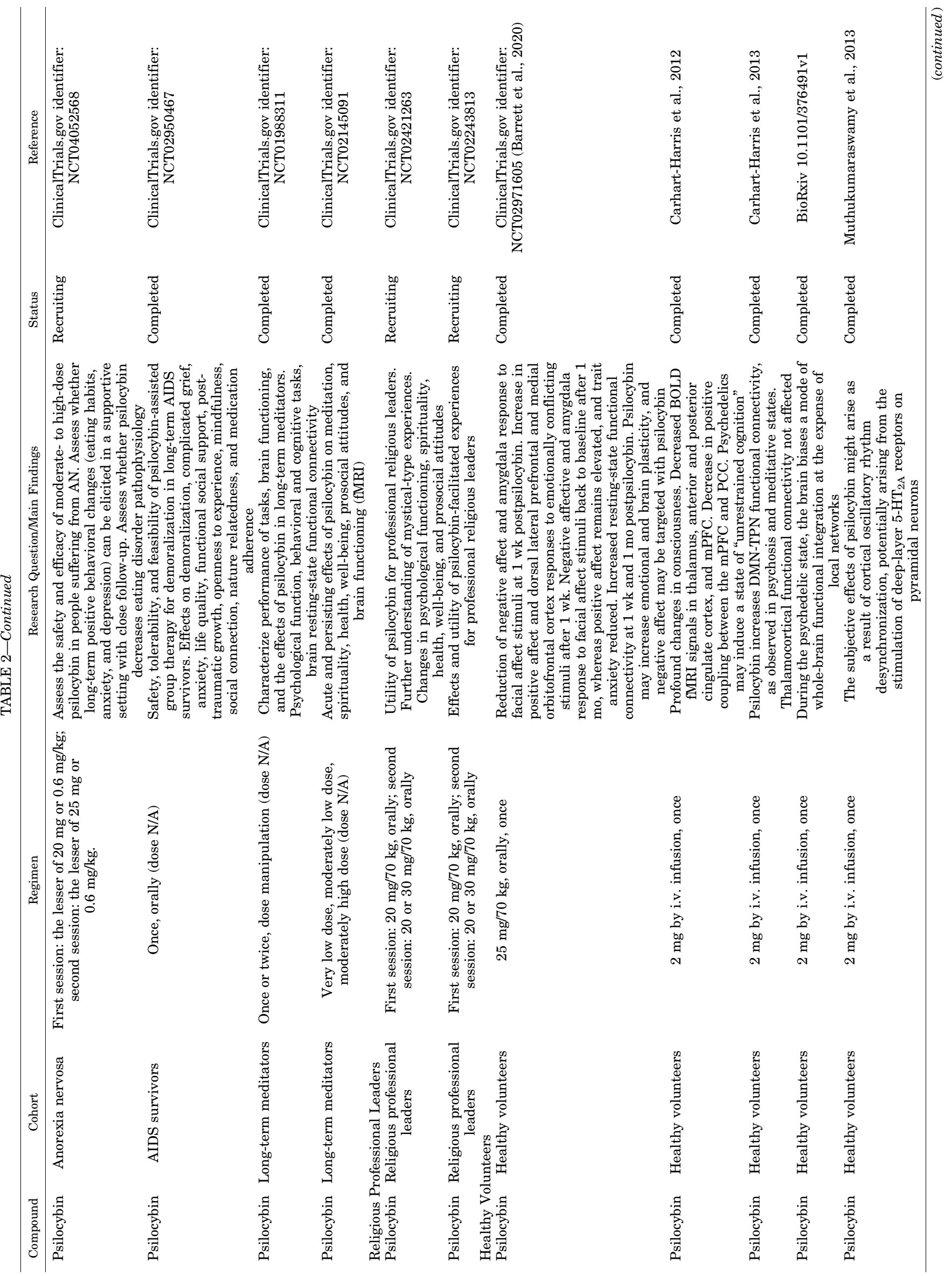




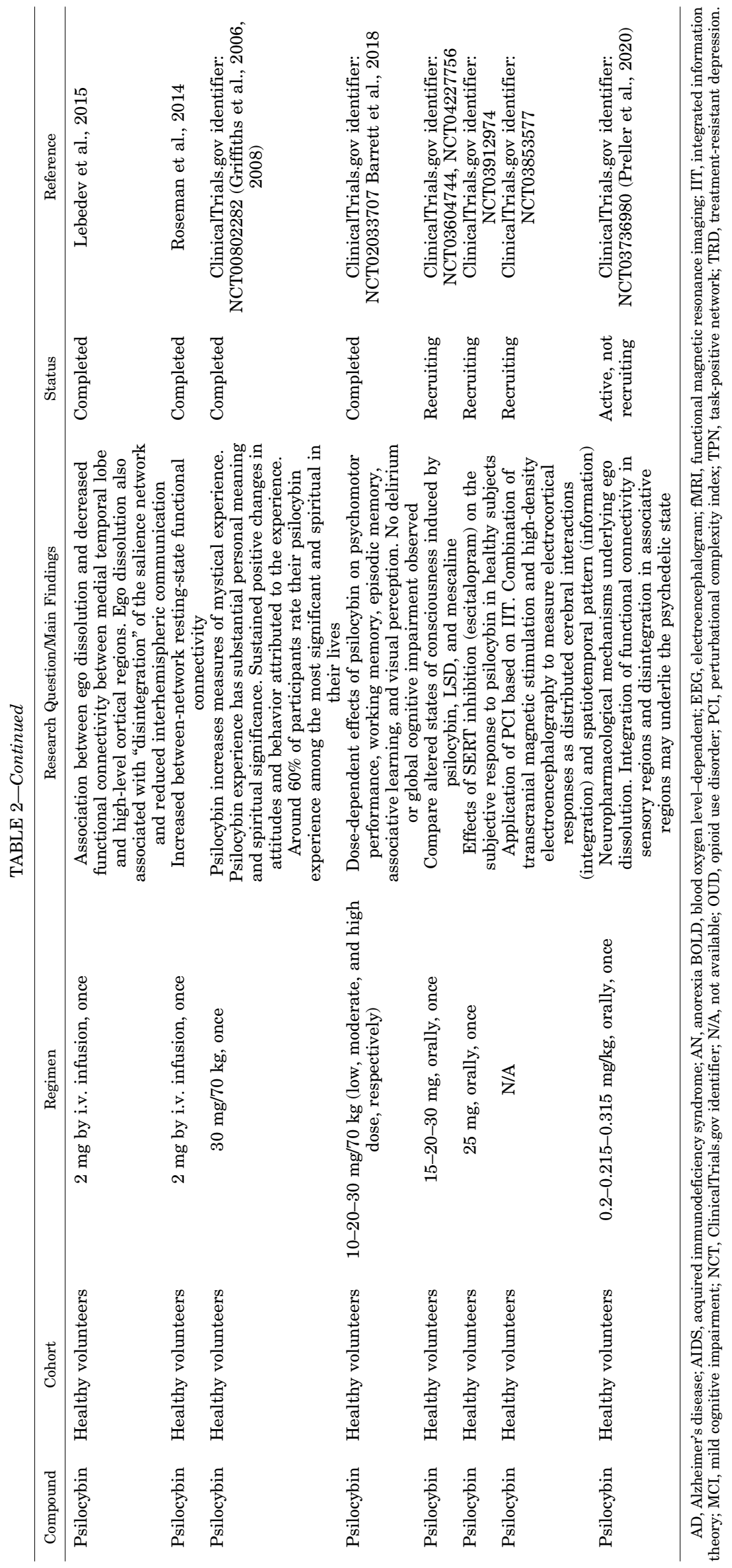




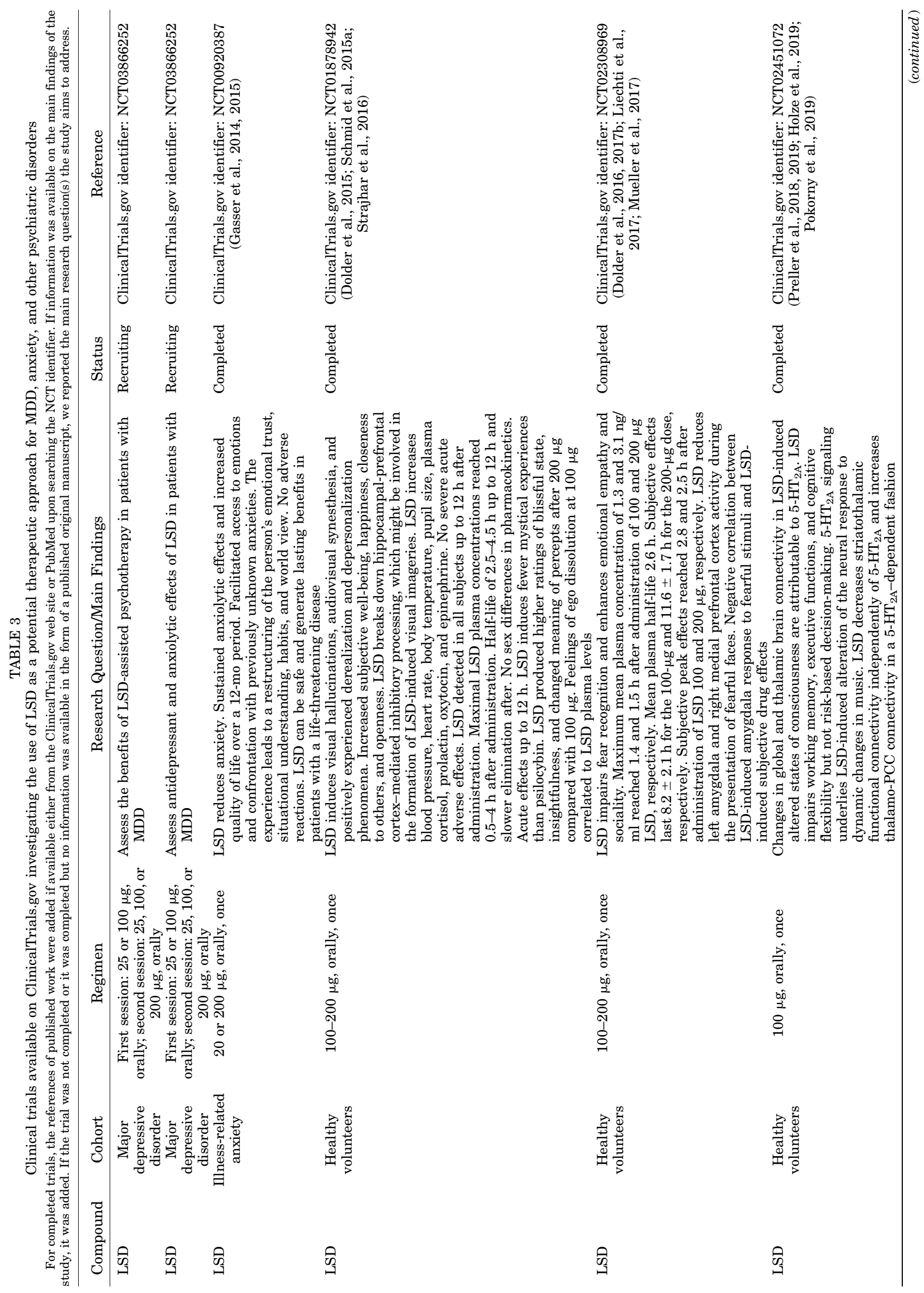




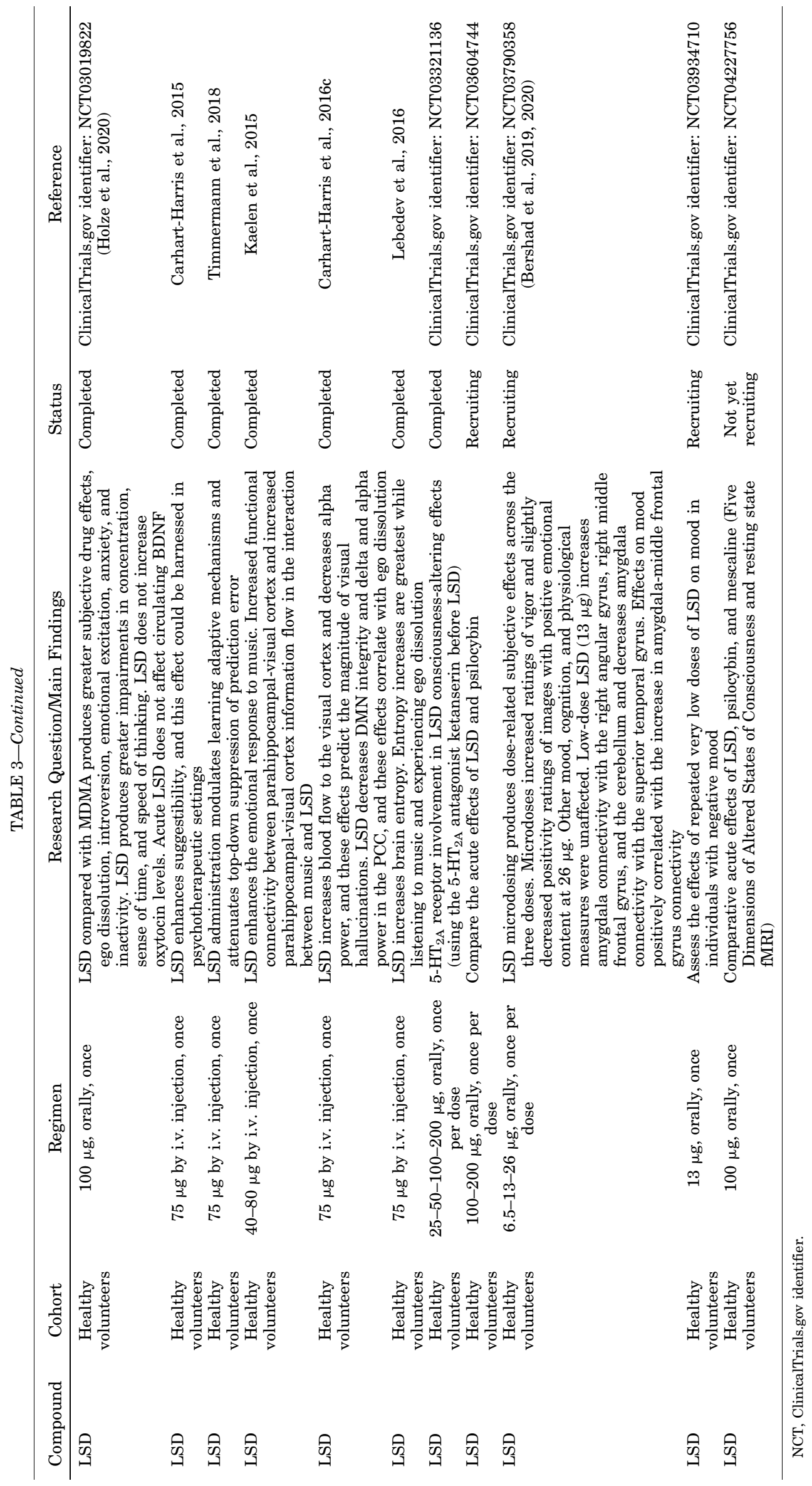




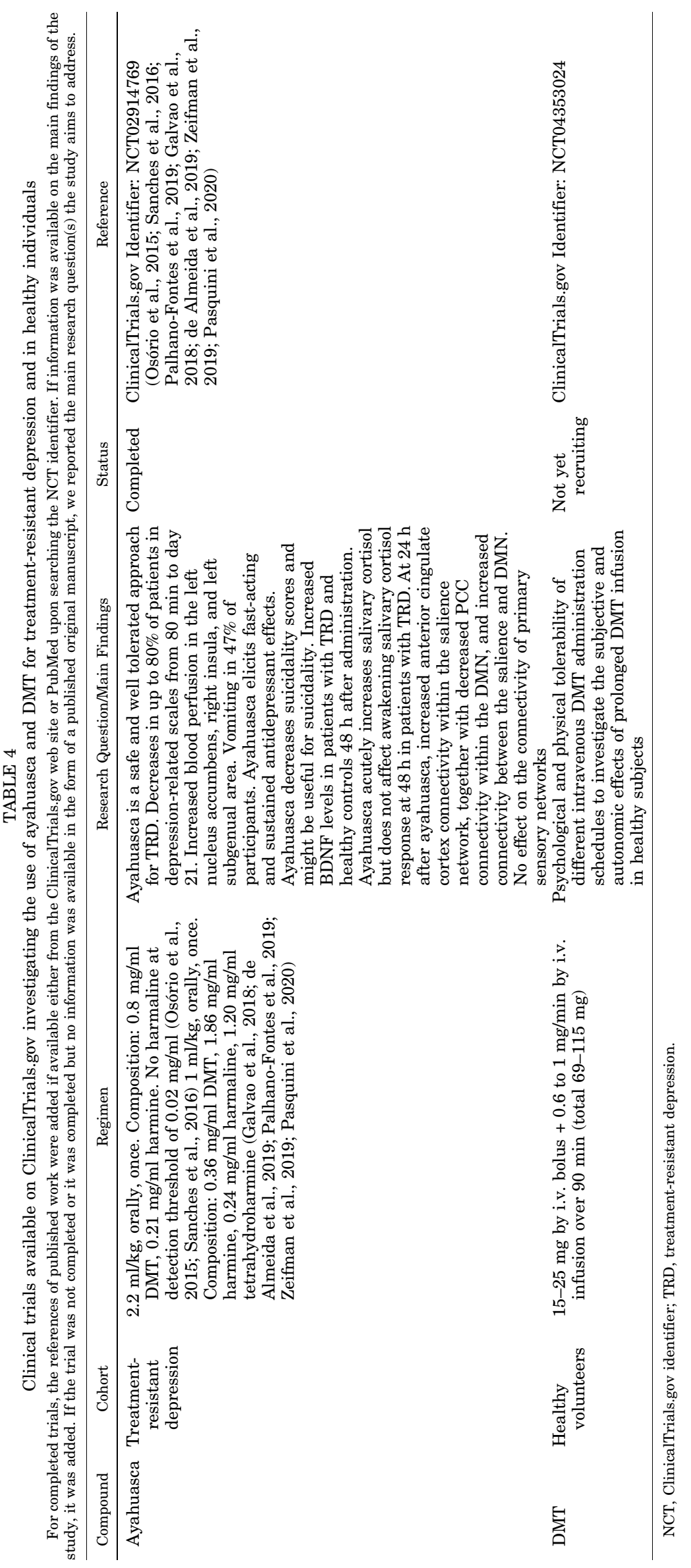


Nuyen et al., 2006). This suggests that the potential therapeutic reach of psychedelic compounds might extend to neurodegenerative states and potentially elderly populations with neurodegenerative disorders (Family et al., 2020). Future clinical trials should investigate the efficacy and potential side effects profile of a similar therapeutic approach in elderly populations with neurodegenerative conditions.

BDNF represents a prototypical neurotrophin in the brain that interfaces stress susceptibility, psychiatric disorders, and antidepressant response (Shimizu et al., 2003a; Martinowich et al., 2007; Martinowich and Lu, 2008). For example, decreased BDNF levels are consistently observed across psychiatric disorders such as MDD (Kim et al., 2007), PTSD (Dell'Osso et al., 2009; Angelucci et al., 2014), and BD (Palomino et al., 2006; Reinhart et al., 2015), and low BDNF levels correlate with suicidal behavior (Kim et al., 2007; Lee et al., 2007). Psychotic depression (Lee et al., 2007), but not $\mathrm{SCZ}$, is associated with higher BDNF levels compared with controls (Shimizu et al., 2003b). Not surprisingly, pharmacological antidepressant therapies, sleep, and physical exercise increase BDNF levels (Shimizu et al., 2003a; Lu et al., 2014), and these effects might be mediated at least partially by 5-HT2A signalling in the hippocampus and the neocortex (Vaidya et al., 1997). In terms of predictive biomarkers of treatment response, higher baseline BDNF levels correlate with greater depression improvement after SSRIs (Wolkowitz et al., 2011). Similarly, PTSD is characterized by low BDNF levels (Angelucci et al., 2014), and genetic variability within the BDNF gene confers susceptibility or resilience to PTSD (Hemmings et al., 2013; Zhang et al., 2014). It has also been suggested that BDNF may be a common denominator in PTSD-BD comorbidity (Kauer-Sant'Anna et al., 2007; Rakofsky et al., 2012). Preclinical models indicate that individual susceptibility to PTSD might result from sustained transcriptional downregulation of BDNF (Kozlovsky et al., 2007), which might be mediated by epigenetic processes such as DNA methylation and histone acetylation (Roth et al., 2011) and an increased expression of the BDNF receptor, the neurotrophic receptor tyrosine kinase 2 (NTRK2, also known as tropomyosin related kinase B [TrkB]), in brain areas susceptible to stress, such as the cornu ammonis (CA) 1 region of the hippocampus (Kozlovsky et al., 2007).

\section{B. Effects of Psychedelic Compounds on Neuronal and Synaptic Plasticity}

BDNF enhances AMPA receptor delivery to the synapse (Caldeira et al., 2007), and this mechanism might be involved in the psychedelic-induced, BDNFmediated enhancement of neural plasticity elicited by psychedelic compounds (Ly et al., 2018). This possibility is supported by the fact that AMPA receptor potentiation is beneficial in the treatment of psychiatric disorders (Zarate and Manji, 2008) via enhancing neuroplastic pathways (Seo et al., 2020). Importantly, given that AMPA receptors mediate the formation of neural networks during development (O'Neill et al., 2004), this effect might be involved in the reported "resetting" feelings experienced by individuals who ingest psychedelics (discussed in the Going Beyond Receptors: Neuronal Circuits Activated by Psychedelic Drugs section) (Carhart-Harris et al., 2017). Stimulation of neurotrophic pathways, leading to increased neurogenesis, cognitive flexibility, and ultimately changes in behavior, has long represented a desirable characteristic in psychiatric drugs, and the neuroplastic and cognitionenhancing effects elicited by psychedelics could be exploited to achieve such outcomes.

Psychedelics elicit profound changes in neuronal and synaptic plasticity through mammalian target of rapamycin (mTOR) and BDNF-NTRK2 signaling (see Fig. 1 and Table 5 for details) (Li et al., 2010; Dakic et al., 2017; Ly et al., 2018). The neuroplasticity-enhancing effects of serotonergic psychedelics resemble those of the dissociative anesthetic ketamine, which boost neural and synaptic plasticity, at least partially, via potentiating BDNF and mTOR signaling ( $\mathrm{Li}$ et al., 2010; Ly et al., 2018). Given these similarities, it seems likely that the stimulation of neurotrophic pathways by psychedelics is involved in the psychiatric improvements observed in clinical trials (Berman et al., 2000; Carhart-Harris et al., 2017; Mithoefer et al., 2018; Palhano-Fontes et al., 2019).

1. Ketamine. Subanesthetic doses of ketamine elicit rapid, robust, and sustained antidepressant effects (Berman et al., 2000; Price et al., 2009; Murrough et al., 2013a) at least partially via activating mTOR (Li et al., 2010), a key intracellular signaling pathway altered in MDD and other psychiatric disorders, such as ASD and SCZ (Li et al., 2010; Jernigan et al., 2011; Costa-Mattioli and Monteggia, 2013). In turn, this leads to the BDNFmediated stimulation of dendritic, synaptic, and neuronal plasticity, which may well translate to network plasticity (Murrough et al., 2013b; Choi et al., 2017; Ly et al., 2018). Ketamine treatment increases BDNF levels in the prefrontal cortex (PFC) and CA3 and dentate gyrus regions of the hippocampus, and the latter region is intimately involved with adult neurogenesis (Dong et al., 2017). Moreover, ketamine treatment during stress exposure prevents the dendritic spine density loss observed in the CA3 region and dentate gyrus of the hippocampus of controls subjects (Dong et al., 2017). Similarly, ketamine increases spine density and synaptic plasticity markers such as synap$\sin 1$, postsynaptic density protein 95 , and mGluR1 in rats exposed to stress (Sarkar and Kabbaj, 2016).

Ketamine also interacts with $\sigma 1$ receptor (S1R, discussed in the immunomodulation section) and S2R, key signal transducers of neuroplastic and neurotrophic pathways that at least partially mediate the therapeutic improvements elicited by currently approved 


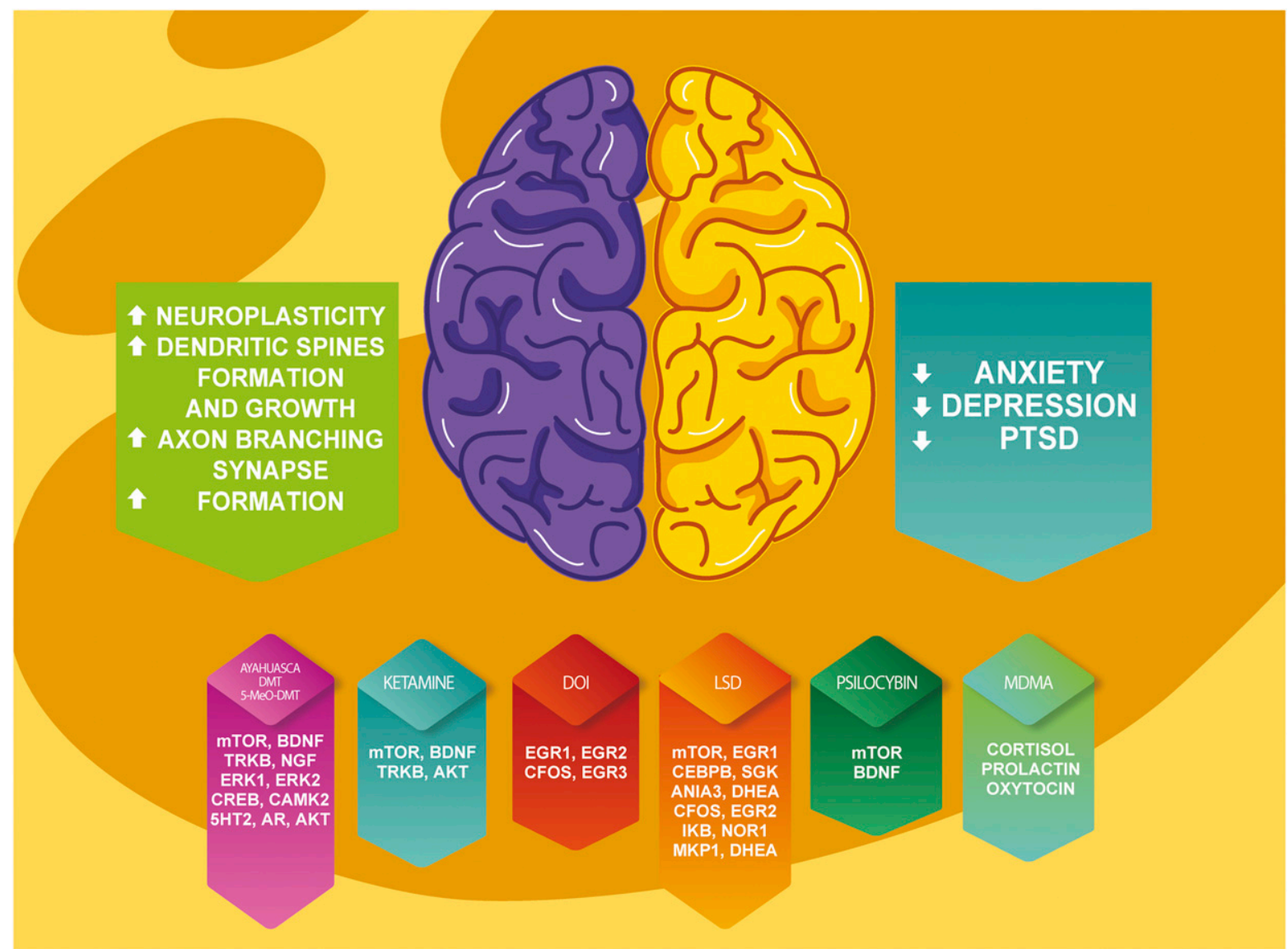

Fig. 1. Neuroplastic effects of psychedelic compounds relevant to psychiatric disorders and comorbidities. The main outcomes elicited by psychedelic compounds on neuroplastic and neurogenesis-related pathways are reported in the green box (purple brain). The resulting outcomes on psychiatric symptoms are reported in the blue box (orange brain). For each compound, or group of compounds with similar pharmacology, the main classes of transcription factors and signaling pathways activated that are thought to mediate the effects of the compound on synaptic and neuronal plasticity are reported. In the bottom part of the figure, the main receptors involved in signal transduction for each compound or group of compounds with similar pharmacology and the resulting neurotransmitter released are reported. AR, androgen receptor; ALT, alternative lengthening of telomeres; ANIA3, activity and neurotransmitter-induced early gene 3; CAMK2, calcium/calmodulin-dependent protein kinase 2; CEBPB, CCAAT/enhancer-binding protein beta; COMT, catechol O-methyltransferase; CREB, cAMP response element-binding protein; MKP1, mitogen-activated protein kinase phosphatase; NOR1, neuron-derived orphan receptor-1; IKB, inhibitor of kB kinase.

antidepressant medications (Yagasaki et al., 2006; Dhir and Kulkarni, 2007; Fishback et al., 2010; Fujimoto et al., 2012; Krystal et al., 2019). For this reason, S1R and S2R may be involved in ketamine's antidepressant action. $(R)$-Ketamine possess higher affinity for S1R than its enantiomer $(S)$-ketamine, and S1R antagonism does not decrease the antidepressant effects of ketamine (Robson et al., 2012). However, S1R antagonism does decrease the nerve growth factor (NGF)-mediated neuroplastic effects of ketamine. These results demonstrate that the ketamineinitiated, S1R-mediated modulation of NGF might be involved in the antidepressant effects elicited by ketamine (Fujimoto et al., 2012; Yagasaki et al., 2006; Krystal et al., 2019). Lastly, low-dose ketamine increases hippocampal AMPA/NMDA receptor density ratio in a preclinical MDD model (Tizabi et al., 2012). Therefore, decreased AMPA/NMDA receptor density ratio could be involved in the pathogenesis of MDD (Aleksandrova et al., 2017), and ketamine might alter this ratio to ultimately enhance synaptogenesis via upregulating mTOR/BDNF signaling (Tizabi et al., 2012; Aleksandrova et al., 2017).

2. N,N-Dimethyltryptamine, 5-Methoxy-N,N-dimethyltryptamine, and Ayahuasca. Neuroplastic effects have been observed in response to ayahuasca. For example, a single dose of ayahuasca increases circulating BDNF levels in healthy controls and patients with treatmentresistant depression (de Almeida et al., 2019). Preclinical findings support that neuroplasticity-enhancing effects might mediate therapeutic improvements in response to psychedelics. For instance, repeated administration of ayahuasca increases hippocampal BDNF levels in female rats (Colaço et al., 2020). Moreover, a single dose of $5-\mathrm{MeO}-\mathrm{DMT}$ increases neurogenesis in the dentate gyrus of the adult mouse (Lima da Cruz et al., 2018). This is of particular relevance given that antidepressantfree patients with depression have decreased BDNF levels (Diniz et al., 2010), and antidepressants increase BDNF (Aydemir et al., 2005), thereby enhancing hippocampal neurogenesis (Anacker et al., 2011). Similarly, other nonpsychedelic S1R agonists suppress 


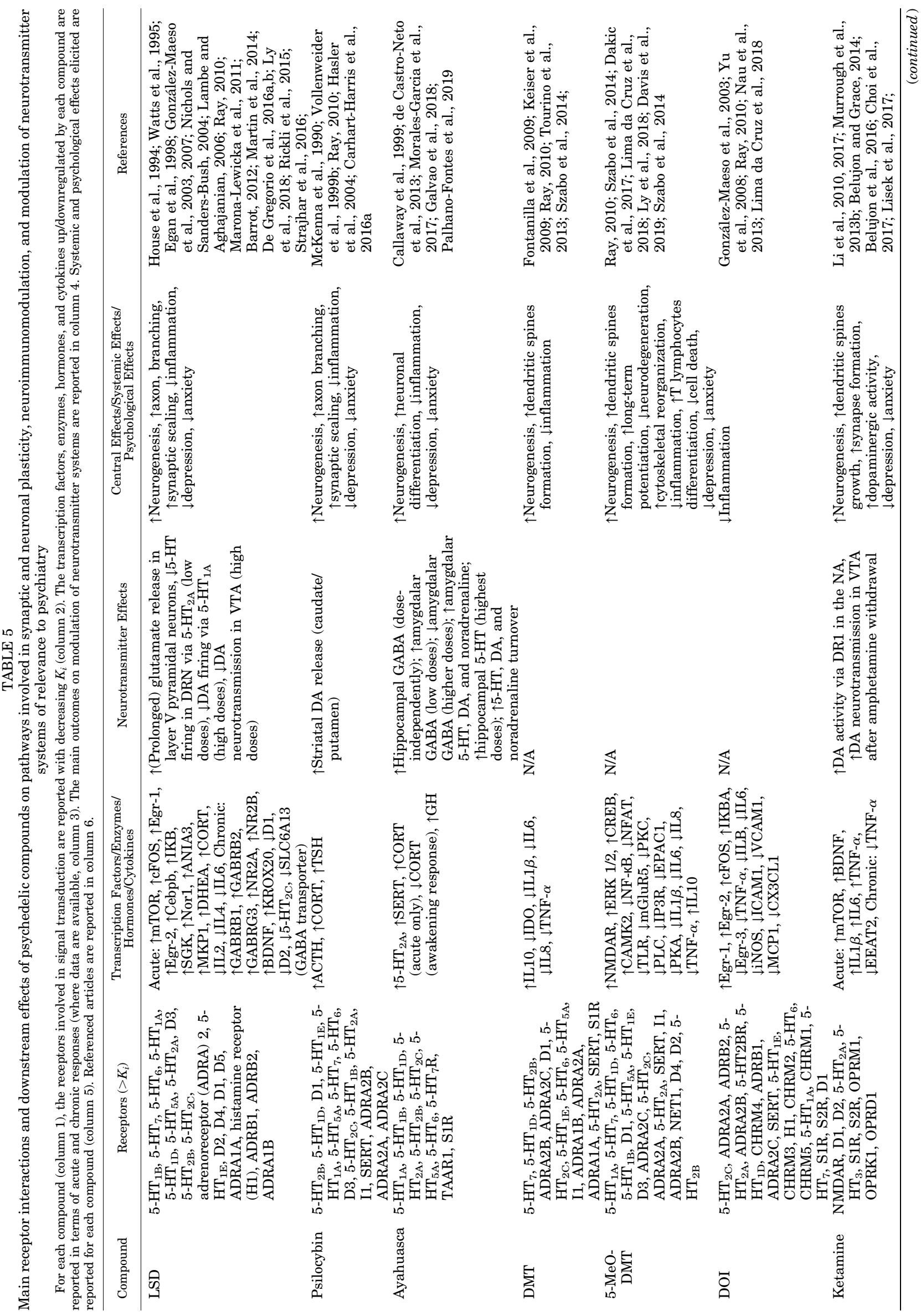




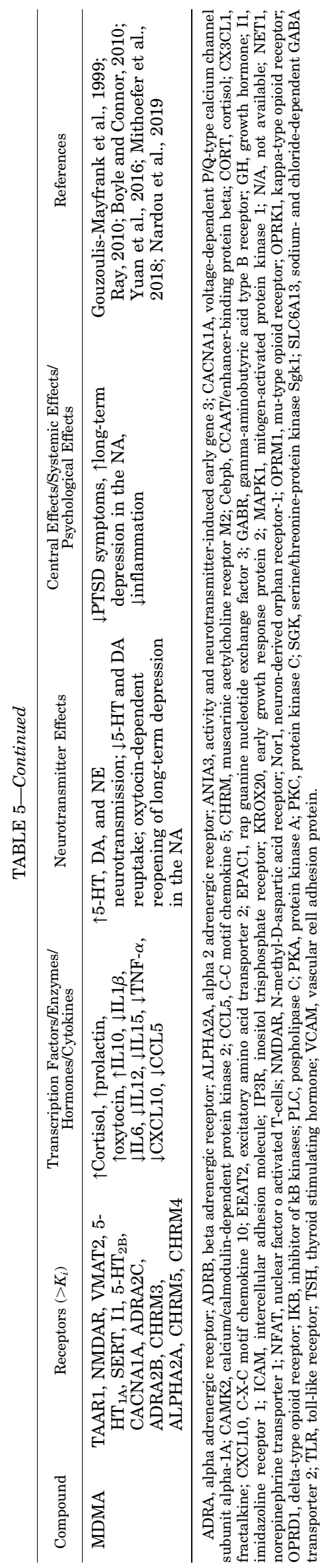

neurodegeneration and attenuate disease progression in preclinical models via boosting neuronal proliferation and maturation (Ono et al., 2014), increasing hippocampal BDNF, and activating antioxidant pathways (Kikuchi-Utsumi and Nakaki, 2008; Pal et al., 2012).

S1R modulation might represent a synergistic mechanism to $5-\mathrm{HT}_{2 \mathrm{~A}}$ receptor modulation by psychedelics, which mediates the upregulation of neurotrophic factors. In fact, S1Rs are enriched in brain areas involved with cognition, stress, and psychiatric disorders, and their stimulation induces adaptive neuroplasticity (Hindmarch and Hashimoto, 2010); psychedelic and nonpsychedelic S1R agonists ameliorate psychiatric symptoms (Hindmarch and Hashimoto, 2010; Davis et al., 2019; Palhano-Fontes et al., 2019). The other active compounds contained in ayahuasca are the $\beta$-carbolines harmol, harmine, harmaline, and tetrahydroharmine (found in B. caapi), which inhibit the metabolism of DMT to render it orally active. These compounds also elicit neuroplastic effects. In vitro studies found that these molecules induce differentiation, proliferation, and migration of neural precursors (Morales-Garcia et al., 2017). This strengthens the notion that ayahuasca alkaloids might be useful for neurodegenerative states. Contrasting preclinical evidence suggests that repeated DMT might hinder synaptic plasticity (a sex-specific effect in females) (Cameron et al., 2019). Although preliminary, these results highlight the need for further preclinical studies with greater statistical power to identify potential neuroplasticity-related side effects.

3. Lysergic Acid Diethylamide. It was recently reported that microdoses of LSD increase circulating BDNF levels in healthy volunteers, suggesting that LSD might have neurotrophic effects in humans (Hutten et al., 2020). Another study investigated whether LSD produces acute gene expression changes in peripheral blood mononuclear cells 1.5 and 24 hours after administration, with no detected changes in 5-HT2A receptor or EGR-1, -2, and -3 transcript levels (Dolder et al., 2017a). In rodents, LSD upregulates CCAAT/enhancer-binding protein $-\beta$ in the prefrontal cortex (Nichols and SandersBush, 2004). This transcription factor affects synaptic scaling, an essential "housekeeping" neuronal process that modulates synaptic function (Turrigiano, 2008). Consistent with neuroplastic and neuroprotective outcomes, LSD increases cFOS, Egr-1, and Egr-2 in murine primary neuronal cultures (González-Maeso et al., 2003, 2007; Li et al., 2005). Moreover, LSD increases dehydroepiandrosterone (DHEA), the most abundant neurosteroid in the central nervous system (Strajhar et al., 2016). Similar to DMT (Fontanilla et al., 2009), DHEA is an S1R agonist, and signaling of DHEA and pharmacological compounds at S1R stimulates synaptic activity and neurogenesis and ameliorates druginduced cognitive impairments (Meunier and Maurice, 2004; Moriguchi et al., 2013). This indirect action of LSD on S1R might be involved in the clinical improvements 
elicited by LSD (Moriguchi et al., 2013; Szabo et al., 2014; Schmidt et al., 2016). S1R forms heterodimers with D2 receptors, and such interaction may also boost neurogenesis (Beggiato et al., 2017). Lastly, DOI, a close relative of LSD, rapidly increases spine density growth in cortical cells, activating a synaptogenic pathway mediated by kalirin 7, while increasing Egr-1 in the mouse somatosensory cortex (Jones et al., 2009). Given that the EGR family of transcription factors is involved in synaptic plasticity, neurogenesis, and the pathologic processes underlying psychiatric symptoms, the effects of psychedelics on EGR-mediated transcription should be further elucidated (González-Maeso et al., 2003, 2007; Clark et al., 2010; Duclot and Kabbaj, 2017).

4. Psilocybin. A recent study reported that psilocybin modulates neurotrophic-related gene expression in the PFC and hippocampus, with a preferential effect on the PFC, but inducing changes which are also appreciable in the hippocampus (Jefsen et al., 2020). More specifically, in the PFC psilocybin increased the expression of CEBPB, c-Fos, dual specificity protein phosphatase 1 (Dusp1), transcription factor jun- $\beta$, NF-kappa- $\beta$ inhibitor- $\alpha$ (Ік $\beta \alpha)$, nuclear receptor subfamily 4 group A member 1 , while the serine/threonine-protein kinase 1 (Sgk1), protein fosB, protein S100-A10, and postsynaptic density protein 95 were increased only response to some doses of psilocybin. Dual specificity protein kinase (CLK1) was dose dependently decreased in response to psilocybin (Jefsen et al., 2020). In the hippocampus, Dusp1, I $\beta \alpha$, and Sgk 1 transcripts were similarly increased by acute psilocybin and Clk1 expression was strongly decreased (Jefsen et al., 2020). Psilocybin affects hippocampal neurogenesis in a biphasic fashion (Catlow et al., 2013). Although at lower doses $(0.1 \mathrm{mg} / \mathrm{kg})$ a nonstatistically significant trend was observed toward increased neurogenesis, higher doses (1-5 mg/kg) decreased neurogenesis 2 weeks after treatment (Catlow et al., 2013). These neuroplastic changes were associated with a facilitation of fear extinction at low doses, hypothetically mediated by a psilocybin-induced, 5-HT-mediated DA enhancement, an effect known to facilitate fearextinction learning (Borowski and Kokkinidis, 1998; Catlow et al., 2013).

5. 3,4-Methylenedioxymethamphetamine. The debate on the neurotoxicity of MDMA creates a longstanding divide (Mithoefer et al., 2003; Parrott, 2013, 2014; Doblin et al., 2014; Pantoni and Anagnostaras, 2019; Ricaurte et al., 2002 [retracted]). Most of the available early preclinical research is focused on the neurotoxic effects of MDMA, which may explain cognitive impairments and psychiatric sequelae in MDMA abusers (Parrott, 2001, 2013). To simulate binge abuse and the resulting neurotoxic effects, relatively high doses (often in a chronic-administration design) were administered in these studies, with considerable neurotoxicity (Pantoni and Anagnostaras, 2019). Indeed, human studies indicate damaging effects on SERT homeostasis in heavy MDMA users (Baumann et al., 2007; Müller et al., 2019). However, although these studies represent a valid paradigm for MDMA abuse, they do not seem to adequately model the sparing use of relatively low doses employed in MDMA-augmented psychotherapy (Amoroso, 2019; Pantoni and Anagnostaras, 2019), which elicits notable improvements in treatmentrefractory PTSD symptoms (Mithoefer et al., 2011, 2013; Mithoefer et al., 2018; Feduccia et al., 2019; Bahji et al., 2020).

Importantly, metabolites from MDMA metabolism seem to be responsible for the neurotoxic effects of MDMA given that direct intracerebroventricular administration of MDMA does not elicit neurotoxicity (Green et al., 2003). Accordingly, intrastriatal administration of 2,4,5-trihydroxymethamphetamine significantly depletes both 5-HT and DA, intracortical administration decreases 5-HT, and intracerebroventricular administration moderately depletes striatal DA without affecting 5-HT levels (Johnson et al., 1992; Zhao et al., 1992). Other metabolites, such as 2-hydroxy4,5(methylenedioxy)methamphetamine (6-HO-MDMA), appear to be nontoxic given that intrastriatal and intracerebroventricular administration does not affect 5-HT or DA levels (Zhao et al., 1992). Such metabolites are quinone-thioethers, orto-quinones, and the glutathione conjugates 5-(glutathion-Syl)- $\alpha$-methyldopamine (5-GSyl$\alpha$-MeDA) and 2,5-bis-(glutathion-S-yl)- $\alpha$-methyldopamine (2,5-bis-(glutathione-S-yl)- $\alpha$-MeDA) (Miller et al., 1996; Bai et al., 1999; Monks et al., 2001; Green et al., 2003).

The existing lack of clear knowledge on potential neurotoxic effects of MDMA could be filled by employing realistic preclinical models that simulate clinically relevant pharmacodynamics (Pantoni and Anagnostaras, 2019; Vollenweider et al., 1999a). If further studies will determine that neurotoxic effects are elicited by MDMA administration in humans at clinically relevant doses, strategies could be implemented in an effort to protect against these effects (Tourino et al., 2010).

\section{Psychedelic Compounds as Immunomodulatory and Anti- Inflammatory Agents}

\section{A. Inflammation}

Inflammation entails a strong but short-lived cascade of events that are mobilized in response to stressful stimuli with the ultimate goal of dealing with the stressor and returning the system to homeostasis. Although a spatiotemporally fine-tuned inflammatory response is key to physical and psychological defense and for tissue repair and remodeling, it presents collateral damage potential if the process is too violent or if it does not reach resolution within a reasonable spatiotemporal frame (Kotas and Medzhitov, 2015; Hotamisligil, 2017). Activation of peripheral inflammatory pathways leads to the activation of central 
inflammatory cascades directly via 1) circulation of inflammatory mediators in central nervous system lymphatic vessels, 2) active transport and/or compromised blood-brain barrier, and 3) crossing at circumventricular organs (Maier and Watkins, 2003; Banks, 2005; Dantzer et al., 2008; Louveau et al., 2015) and indirectly via stimulating the de novo production of cytokines in the brain (Hanamsagar et al., 2012; Weber et al., 2015; Inserra et al., 2019).

\section{B. Inflammation-Induced Psychiatric Symptoms}

Increased proinflammatory signaling in the brain results in the development of "depressive-like" symptoms, a behavioral repertoire encompassing anxiety, low motivation, fatigue, loss of interest, inability to seek and experience pleasure, exaggerated pain responses, lack of concentration, and sleep pattern alterations, manifestations that closely resemble clinical MDD symptomatology (Dantzer et al., 2008). Supporting an involvement of the immune system in the development of psychiatric symptoms, therapies involving the administration of inflammatory molecules (such as interferon- $\alpha$ ) and polymorphisms in inflammationrelated genes increase the susceptibility to psychosocial stress via affecting immune signaling (Wong et al., 2008; Lotrich, 2009). On the other hand, patients experiencing depressive, PTSD, or BD symptoms present dysregulated reactivity of immune cells accompanied by increased levels of central proinflammatory mediators such as IL1 $\beta$, IL6, TNF- $\alpha$, C-reactive protein, and the translocator protein (TSPO), some of which correlate with symptom severity (Rohleder et al., 2004; Dowlati et al., 2010; Setiawan et al., 2015; Lindqvist et al., 2017; Zou et al., 2018) [meta-analyses: (Modabbernia et al., 2013; Passos et al., 2015; Baumeister et al., 2016; Goldsmith et al., 2016; Leighton et al., 2018)]. Psychiatric patients also present abnormal functioning of the hypothalamic-pituitary-adrenal (HPA) axis, one of the most important stress-responsive systems, which increases inflammation upon activation by psychosocial and physical stressors (Melhem et al., 2016; Keller et al., 2017; Dunlop and Wong, 2019).

Patients diagnosed with MDD and PTSD present a systemic low-grade chronic inflammatory state driven by a shift of immune responses toward T-helper (Th) 1 and decreased T-regulatory cell activity (Myint et al., 2005; Gola et al., 2013; Alcocer-Gómez et al., 2014). Such a shift signifies an increase in cell-mediated immune responses and a decrease of humoral immune responses, events which create a proinflammatory milieu that may lead to, or be a consequence of, psychiatric disorders (Myint et al., 2005; Dantzer et al., 2008; Gola et al., 2013; Alcocer-Gómez et al., 2014). Given that in psychiatric disorders there is an imbalance in this equilibrium that is shifted toward Th1 (proinflammatory) responses, therapeutic approaches with the ability to shift this balance in favor of an enhancement of Th2 (immunomodulatory and anti-inflammatory) responses have long been sought and may help ameliorate the psychiatric symptomatology (Pulendran, 2004; Myint et al., 2005; Gola et al., 2013; Alcocer-Gómez et al., 2014). This dysregulated immune milieu is accompanied by pathologic fluctuations in gut microbiome composition that further fuel inflammation, increasing the likelihood of developing comorbid systemic illnesses (Rogers et al., 2016; Inserra et al., 2018; Cheung et al., 2019). The changes elicited by psychedelics over immune function represent a marked shift of inflammatory responses from Th1 to Th2 responses, which overall denotes a shift from proinflammatory to anti-inflammatory and immunomodulatory effects (Romagnani, 1997; Maldonado-López and Moser, 2001; Pulendran, 2004).

\section{Anti-Inflammatory Therapies in the Treatment of Psychiatric Disorders}

Anti-inflammatory treatments are being tested as adjunctive therapies in psychiatry, but with mixed results. For example, minocycline, a second-generation, semisynthetic tetracycline with anti-inflammatory properties, seems to be a promising adjunctive in patients with MDD, SCZ, and BD and dysregulated inflammation, potentially via its acute effects on inflammatory mediators and long-term effects over gut microbiome composition (Chaudhry et al., 2012; Wong et al., 2016; Dean et al., 2017). Clinical trials partly confirmed the antidepressant effects of minocycline, suggesting it might be a useful first-line or adjunct therapy in psychiatric patients with high baseline inflammatory markers (Raison et al., 2006; Miyaoka et al., 2012; Raison and Miller, 2013; Dean et al., 2017; Rosenblat and McIntyre, 2018). Not surprisingly, classic antidepressant and anti-inflammatory therapies similarly reduce signals of immune activation (such as IL1 $\beta$, IL2, IL6, and TNF- $\alpha$ ), and higher TNF- $\alpha$ levels predict an increased likelihood of treatment resistance (Köhler et al., 2014; Strawbridge et al., 2015). Also relevant for this review, nonpsychedelic S1R agonism inhibits Th1 responses by suppressing interferon- $\gamma$ (IFN- $\gamma$ ), granulocyte-macrophage colony-stimulating factor and TNF- $\alpha$ and increasing the anti-inflammatory IL10 (Carayon et al., 1995; Zhu et al., 2003). However, clinical studies with anti-inflammatory agents are still under investigation, since a few produced negative results in depression [including the TNF- $\alpha$ inhibitor infliximab (Raison et al., 2013)] or mixed results (Köhler-Forsberg et al., 2019).

\section{Immunomodulatory and Anti-Inflammatory Pathways Activated by Psychedelics}

Psychedelic compounds activate immunomodulatory and anti-inflammatory programs mediated at least partially by $5-\mathrm{HT}_{2 \mathrm{~A}}$ receptor agonism (see Fig. 2) (Flanagan and Nichols, 2018). Although serotoninmediated $5-\mathrm{HT}_{2 \mathrm{~A}}$ receptor activation is canonically 

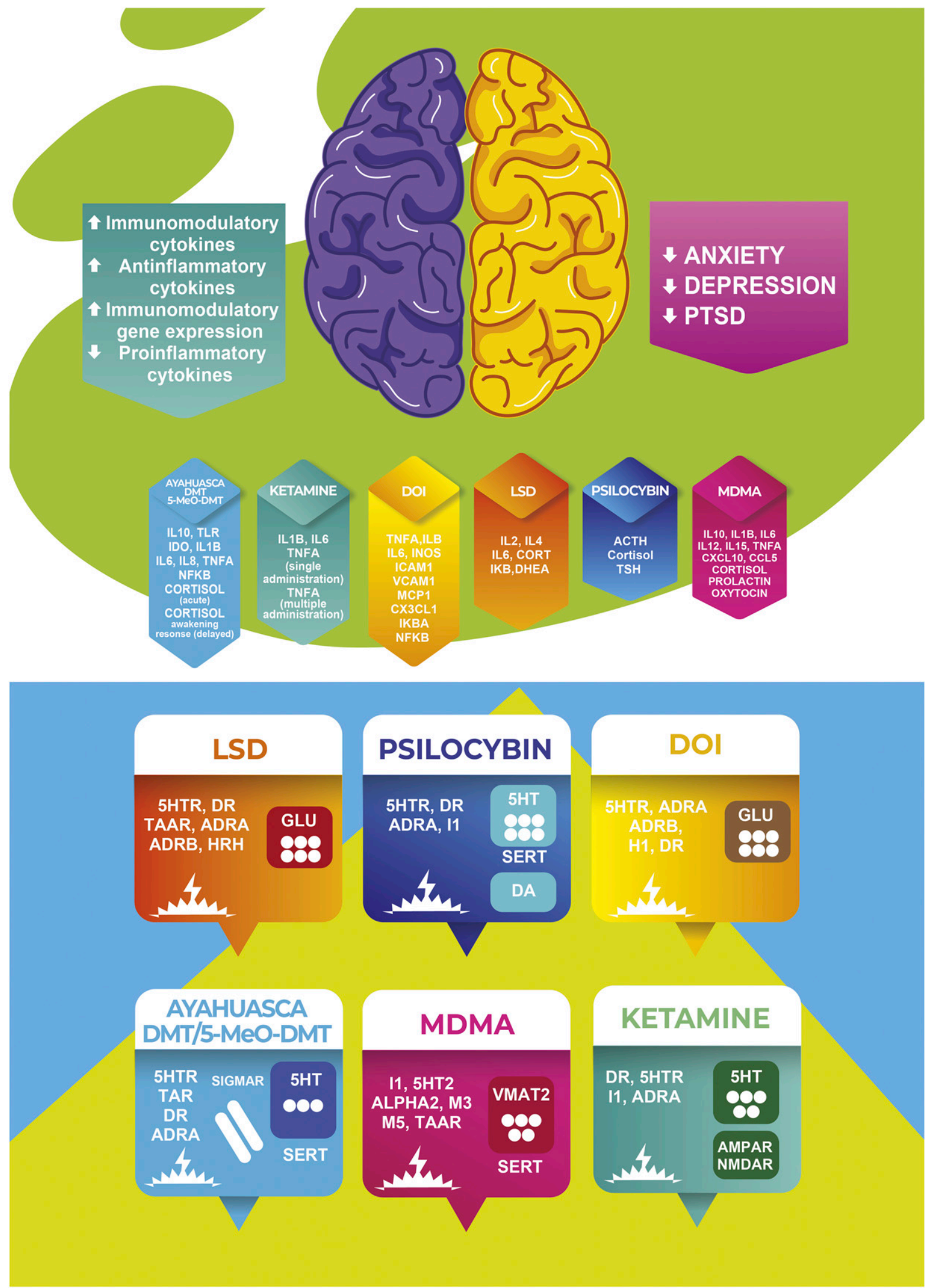

Fig. 2. Anti-inflammatory and immunomodulatory effects of psychedelic compounds relevant to psychiatric disorders and comorbidities. The main outcomes elicited by psychedelic compounds on inflammation and immunity-related pathways are reported in the blue box (purple brain). The resulting outcomes on psychiatric symptoms are reported in the purple box (orange brain). For each compound, or group of compounds with similar pharmacology, the main classes of cytokines, chemokines, hormones, transcription factors, and signaling pathways activated, which are thought to mediate the effects of the compound on inflammation and immunity-related pathways, are reported. In the bottom part of the figure, the main receptors involved in signal transduction for each compound or group of compounds with similar pharmacology and the resulting neurotransmitter 
considered a proinflammatory signal (Shajib and Khan, 2015), psychedelic-induced $5-\mathrm{HT}_{2 \mathrm{~A}}$ receptor activation largely results in the recruitment of anti-inflammatory and immunoregulatory pathways (Nau et al., 2013, 2015; Flanagan and Nichols, 2018; Flanagan et al., $2019 \mathrm{~b})$. Such selectivity of anti-inflammatory over proinflammatory effects likely stems from biased signaling cascades (discussed later in the review) mediated by psychedelic-specific conformational stabilization of the $5-\mathrm{HT}_{2 \mathrm{~A}}$ receptor. This leads to the recruitment of psychedelic-specific anti-inflammatory signal transducers, which involve the inhibition of TNF- $\alpha$ and NF$\mathrm{kB}$-propagated proinflammatory signaling (House et al., 1994; Nau et al., 2013; Flanagan et al., 2019b), outcomes which may prove useful for the treatment of autoimmune conditions (Thompson and Szabo, 2020). Ultimately, this effect elicits psychedelic-specific inflammatory gene expression fingerprints. Given that nearly all immune cells express 5 -HT receptors (such as macrophages, monocytes, eosinophils, dendritic cells, and natural killer cells) (Ahern, 2011; Baganz and Blakely, 2013), it is not surprising that psychedelics profoundly affect inflammation and immunity, and it is seems highly likely that these effects are involved in the anxiolytic and antidepressant effects elicited by psychedelics. Importantly, the anti-inflammatory and immunomodulatory effects of psychedelics suggest that they might be useful in "inflammaging" (conditions caused or exacerbated by age-induced chronic inflammation) (Franceschi et al., 2018; Aday et al., 2020; Family et al., 2020).

1. N,N-Dimethyltryptamine, 5-Methoxy-N,N-dimethyltryptamine, and Ayahuasca. Strong anti-inflammatory effects mediated by psychedelic-induced S1R activation have been described (Szabo et al., 2014, 2016). These effects closely resemble the anti-inflammatory action of some SSRIs, SNRIs, and tricyclic antidepressants, (Kenis and Maes, 2002; Köhler et al., 2014; Strawbridge et al., 2015) and the antidepressant outcome of some antiinflammatory therapies (Kappelmann et al., 2018). For example, DMT and its analog 5-MeO-DMT decrease IL1 $\beta$, IL6, IL8, and TNF- $\alpha$ while increasing IL10 in immune-challenged, human monocyte-derived dendritic cells (Szabo et al., 2014). Similarly, in hypoxic stress-challenged neuronal and microglial cultures, DMT administration enhances cell survival and hypoxic stress-related neuroprotective signaling (Szabo et al., 2016). Accordingly, a single inhalation of 5-MeO-DMT decreases the levels of circulating IL6 (Uthaug et al., 2020). Such fast onset of anti-inflammatory effects is of particular interest and could be harnessed in psychiatry and other areas of emergency medicine-for example, in the treatment of systemic inflammatory response syndrome, also called the "cytokine storm" (Mastronardi et al., 2007; Tisoncik et al., 2012; Szabo et al., 2014, 2016; Mastronardi et al., 2015; Inserra et al., 2019; Mehta et al., 2020; Uthaug et al., 2020). Given that in psychiatric disorders there is an imbalance in immune system equilibria, which are shifted toward proinflammatory responses, therapeutic approaches with the ability to shift this balance in favor of an enhancement of immunomodulatory and anti-inflammatory responses have long been sought in psychiatry (Pulendran, 2004; Myint et al., 2005; Gola et al., 2013; Alcocer-Gómez et al., 2014). Whether psychedelics produce a decrease of proinflammatory cytokines in microglial cells similar to SSRIs and SNRIs, which decrease TNF- $\alpha$ and nitric oxide production and microglial activation, is an intriguing possibility that has been partially demonstrated and that warrants further investigation (Tynan et al., 2012; Szabo et al., 2016).

A study investigating proteomics changes in brain organoids in response to 5-MeO-DMT reported a downregulation of NF-kB and nuclear factor of activated $\mathrm{T}$ cells via toll-like signaling and Gq-coupled receptors (Dakic et al., 2017). Importantly, those molecules represent critical checkpoints of immune homeostasis interfacing inflammatory responses, mood, and psychiatric disorders (Keller et al., 2017; Zorn et al., 2017). Similarly, ayahuasca and DMT modulate cortisol release (Strassman and Qualls, 1994; Dos Santos et al., 2012; Galvao et al., 2018), and ayahuasca normalizes the blunted awakening cortisol responses observed in patients with treatment-resistant depression (Galvao et al., 2018) while decreasing the circulating levels of Creactive protein $(\mathrm{CRP})$ in these patients (Galvão-Coelho et al., 2020). Such an HPA axis modulation is quite remarkable given the well characterized dysregulation in psychiatric disorders and the long-sought possibility of modulating the HPA axis to ameliorate psychiatric symptoms (Carvalho and Pariante, 2008; Pariante and Lightman, 2008; Heim et al., 2008; Holsen et al., 2013; Keller et al., 2017).

2. Psilocybin. Psilocybin was shown to acutely increase the circulating levels of the stress-responsive adrenocorticotropic hormone (ACTH) and cortisol, as well as prolactin and thyroid-stimulating hormone (Hasler et al., 2004). The increase in the levels of these hormones was not correlated with stress-induced symptoms, such as anxiety, leading the authors to speculate that the observed temporary increase was likely due to a transient 5- $\mathrm{HT}_{2 \mathrm{~A}}$ receptor-mediated activation of the

released are reported. ADRA, alpha adrenergic receptor; ADRB, beta adrenergic receptor; ALPHA2, alpha 2 adrenergic receptor; CCL5, C-C motif chemokine 5; CORT, cortisol; CX3CL1, fractalkine; CXCL10, C-X-C motif chemokine 10; GLU, glutamate; HRH, histamine receptor; I1, imidazoline receptor 1; IKBA, NF-kappa-B inhibitor alpha; ICAM1, intercellular adhesion molecule 1; M3, muscarinic receptor subtype 3; M5, muscarinic receptor subtype 5; SIGMAR, sigma receptor; TAAR, trace amine-associated receptor; TLR, toll-like receptor; TSH, thyroid stimulating hormone; VCAM1, vascular cell adhesion protein 1 . 
HPA axis, resulting in an increase in the release of ACTH and circulating cortisol (Van de Kar et al., 2001; Hasler et al., 2004).

3. Lysergic Acid Diethylamide. LSD possesses antiinflammatory activity in vitro. Specifically, LSD downregulates IL2, IL4, and IL6 and upregulates mitogenactivated protein kinase phosphatase-1 (House et al., 1994). Moreover, LSD administration increases circulating cortisol, cortisol, cortisone, corticosterone, prolactin, oxytocin, and epinephrine in humans (Schmid et al., 2015a; Strajhar et al., 2016). No reports are available so far on potential long-term immunomodulatory outcomes of LSD. A recent study investigated the effects of LSD on retinal cells and suggested that LSD might be toxic to retinal cells via releasing proinflammatory cytokines (Hu et al., 2018). However, retinal cells do not adequately model central and peripheral neural networks, and whether the concentrations of LSD employed in this study are reached in retinal cells in vivo remains to be determined. Therefore, these results should be interpreted with caution. Nevertheless, more studies are required to investigate whether LSD administration might be damaging to retinal cells in vivo.

4. 2,5-Dimethoxy-4-iodoamphetamine. DOI has been widely studied for its selectivity as a $5-\mathrm{HT}_{2 \mathrm{~A} / 2 \mathrm{C}}$ receptor agonist and for its powerful anti-inflammatory effects, which can be elicited with very low doses of this compound. DOI binding to the $5-\mathrm{HT}_{2 \mathrm{~A}}$ receptor triggers anti-inflammatory signaling, which is different from 5-HT binding to the same receptor, which elicits proinflammatory signaling (Flanagan and Nichols, 2018). DOI powerfully downregulates inflammation in vitro and in vivo via decreasing TNF- $\alpha, \operatorname{IL} 1 \beta$ and IL6, intracellular adhesion molecule-1, vascular cell adhesion molecule-1, monocyte chemoattractant protein 1 (MCP1), and fractalkine, ameliorating parameters of immune-induced gut pathology (Yu et al., 2008; Nau et al., 2013; Flanagan et al., 2019). Importantly, DOI blocks NF-кB activity and nuclear translocation while decreasing iNOS activity (Miller and Gonzalez, 1998; Yu et al., 2008; Nau et al., 2013). DOI was also shown to be protective in a mouse model of asthma via inhibiting IL3, IL5, IL6, IL13, MCP1, and granulocytemacrophage colony-stimulating factor (Nau et al., 2015). A recent study suggests that the anti-inflammatory effects of DOI could also be harnessed for the treatment of systemic inflammatory conditions, such as high-fat diet-induced cardiovascular dysfunction (Flanagan et al., 2019b). In this study, the authors observed that DOI treatment during diet-induced cardiovascular inflammation in apolipoprotein-E knockout mice lowers total and low-density lipoprotein cholesterol. Moreover, this treatment decreases IL6, TNF- $\alpha$, and vascular cell adhesion molecule-1 in the aortic tissue. Furthermore, DOI decreases the circulating levels of the C-X-C motif chemokine 10 (Flanagan et al., 2019b). Together, these findings suggest that DOI and other serotonergic psychedelics might prove valuable for systemic inflammatory diseases, which are often comorbid with psychiatric symptoms.

5. 3,4-Methylenedioxymethamphetamine. MDMA also possesses immunomodulatory properties that influence both the innate and adaptive arms of the immune system (Boyle and Connor, 2010; Mithoefer et al., 2018). Acute administration in humans increases cortisol and prolactin levels (Gouzoulis-Mayfrank et al., 1999) as well as the inflammatory mediators hydroxyeicosatetraenoic acid, dihydroxyeicosatetraenoic acid, and octadecadienoic acid (Boxler et al., 2018) while decreasing circulating $\mathrm{CD}^{+}$helper T cells and increasing natural killer cells, potentially as a result of increased HPA axis activation (Pacifici et al., 1999, 2000, 2007; Young, 2004). Increases in the production of immunosuppressive Th2 cytokines, such as IL4 and IL10, and a decrease of Th1 cytokines, such as IL2 and IFN- $\gamma$, were also reported (Pacifici et al., 2001). Interestingly, repeated exposure to MDMA decreases the number of PV+ GABA neurons in the hippocampal dentate gyrus, and this effect is attenuated by pretreatment with the anti-inflammatory ketoprofen, suggesting that it might be mediated by cyclooxygenasemediated pathways (Anneken et al., 2013).

Given that PTSD is underlined by a low-grade chronic inflammatory state and a shift of cytokine production from Th2 to Th1 coupled to chronic lymphocyte activation, the immunomodulatory and anti-inflammatory effects of MDMA might relate to PTSD symptom reduction after MDMA-assisted psychotherapy (Wilson et al., 1999; Boyle and Connor, 2010; Yuan et al., 2016; Mithoefer et al., 2018). MDMA also decreases the production of the proinflammatory cytokines IL $1 \beta$, IL6, TNF- $\alpha$, and IFN- $\gamma$ while stimulating the production of the anti-inflammatory IL10 and oxytocin (OT) (Boyle and Connor, 2010; Yuan et al., 2016). Similarly, MDMA inhibits lipopolysaccharide-induced TNF- $\alpha$ secretion (Connor et al., 2000). These lines of evidence suggest that MDMA might decrease the immune reactivity, and therefore the damaging potential, of immune cells in patients with PTSD (which are hyperreactive in this disorder), and such an effect might be involved in reduction of clinical symptoms (Wilson et al., 1999; Mithoefer et al., 2018; Bahji et al., 2020).

6. Ketamine. Ketamine displays a biphasic action on immune responses. Acutely, it upregulates IL1 $\beta$, IL6, and TNF- $\alpha$. Chronically, it decreases TNF- $\alpha$ (BlandinoRosano et al., 2017). Mice exposed to highly immunogenic stressors, such as the bacterial toxin lipopolysaccharide, were shown to have higher survival rates when administered ketamine and decreased levels of the proinflammatory TNF- $\alpha$ and IFN- $\gamma$, which might be responsible for their improved survival rates (Takahashi et al., 2010). Given the direct role of inflammation in psychiatric symptoms, the anti-inflammatory action of ketamine could relate to its beneficial and fast-onset 
antidepressant properties (Dantzer et al., 2008; Hotamisligil, 2017). In fact, TNF- $\alpha$ is depressogenic, and TNF- $\alpha$ antagonism is antidepressant (Raison et al., 2013). The short-term proinflammatory effects elicited by ketamine might activate necroptotic pathways, which are required for brain regeneration, a process that may (hypothetically) mediate fear extinction and memory reconsolidation (Feduccia and Mithoefer, 2018; Lloyd et al., 2019).

Together, this body of evidence suggests that the antiinflammatory and immunomodulatory action of psychedelics might be involved in the antidepressant and anxiolytic effects observed in psychiatric populations via pushing the system toward a homeostatic state and, overall, ameliorating psychiatric symptoms. Future studies should explore whether these immunologic outcomes observed in vitro and in preclinical models translate to human physiology. Safety concerns arising from immunosuppression, even though partial, need to be addressed in immunocompetent and immunodeficient models (Boyle and Connor, 2010).

\section{E. $\sigma 1$ Receptor}

Aside from their well known interaction with the 5HT system, most of the compounds discussed here, such as DMT, 5-MeO-DMT, DOI, ketamine, and MDMA, all bind S1R, a still-mysterious receptor whose origin remains puzzling (Fontanilla et al., 2009; Frecska et al., 2013; Szabo et al., 2014; Kourrich, 2017; Kim and Pasternak, 2018). This interaction contrasts the 5$\mathrm{HT}_{2 \mathrm{~A}}$ receptor-centric approach to psychedelic activity and suggests that the action of these compounds at S1R might be more relevant than previously thought (Brammer et al., 2006; Fontanilla et al., 2009; Ray, 2010; Nguyen et al., 2014; Szabo et al., 2014). S1R is highly expressed in limbic areas of the human brain and in several central and peripheral immunocompetent cells, such as monocyte-derived dendritic cells and microglia, which are involved in innate and adaptive immune responses (Ishikawa et al., 2007; Fujimoto et al., 2012; Frecska et al., 2013; Szabo et al., 2014, 2016). Interestingly, ibogaine is the only psychedelic to have its highest affinity at this receptor (Ray, 2010). Recently, a study found for the first time evidence of endogenously produced DMT in the frontal cortex of rat in normal conditions to similar extents to other monoamines, which might help explain the presence of a DMT receptor in the human brain (Dean et al., 2019). Consistent with the putative role of $\mathrm{S} 1 \mathrm{R}$ as a prosurvival and neuroprotective receptor, and with the putative role of DMT as a stress-related neurotransmitter, the levels of endogenous DMT were reported to increase after cardiac arrest in some cortical areas (Szabo and Frecska, 2016; Szabo et al., 2016; Dean et al., 2019).

$\mathrm{S} 1 \mathrm{R}$ is a protein that can be 1) membrane-bound at the cell membrane, where it interacts with several G protein-coupled receptors (GPCRs) and voltage-gated ion channels, thereby modulating signal transduction and neurotransmission; 2) bound to the mitochondriaassociated endoplasmic reticulum membrane, where it acts as a molecular chaperone and modulates $\mathrm{Ca}^{2+}$ signaling; or 3) bound to chromatin remodeling complexes at the nuclear envelope, where it modulates gene expression (Hayashi and Su, 2007; Kourrich et al., 2013; Balasuriya et al., 2014; Tsai et al., 2015; Beggiato et al., 2017; Feltmann et al., 2018; Ortíz-Rentería et al., 2018). Activation of S1R elicits antiamnesic effects in animal models (Maurice et al., 1998) and enhances synaptic stability and plasticity in vitro (Ryskamp et al., 2019). A modulation of junctional cholinergic and glutamatergic neurotransmission via heteroreceptor-mediated biased signaling in brain areas involved in fear extinction and memory reconsolidation might be involved in mediating these effects (Earley et al., 1991; Mash and Zabetian, 1992; Maurice et al., 1998; Antonini et al., 2009; Woodcock et al., 2015; Beggiato et al., 2017; Feltmann et al., 2018; Inserra, 2018). The other main interesting feature of S1R is its modulatory action over chromatin remodeling complexes, which lead to a rearrangement of gene expression (Demmerle et al., 2012; Tsai et al., 2015). Therefore, the positive outcomes on traumarelated fear-memory extinction and memory reconsolidation elicited by compounds such as ayahuasca and MDMA might result from the activation of this receptor (Feduccia and Mithoefer, 2018; Inserra, 2018; Hake et al., 2019).

$\mathrm{S} 1 \mathrm{R}$ dysregulation is implicated in neurodegenerative and psychiatric disorders, as well as in systemic illnesses [reviewed in Tsai et al. (2009); Kourrich et al. (2012); Szabo and Frecska (2016)], suggesting that therapeutic S1R stimulation could hold promise in the treatment of those disorders (Maurice and $\mathrm{Su}, 2009$; $\mathrm{Su}$ et al., 2016). Indeed, S1R modulation elicits prohomeostatic, neuroprotective, and immunomodulatory outcomes, prompting that this might be the case (Nguyen et al., 2015). For example, 5-MeO-DMT and DMT increase neurogenesis in vitro (Morales García et al., 2017) and in vivo (Morales-Garcia et al., 2020), and ayahuasca acutely increase circulating BDNF levels in humans (de Almeida et al., 2019). Future studies should assess the utility of these compounds in preclinical models of neuroinflammatory and neurodegenerative disorders.

\section{Psychedelic Compounds as Modulators of Neurotransmitter Systems}

\section{A. Neurotransmitter Dysfunctions in Psychiatric Disorders}

Psychiatric disorders present serotonergic, norepinephrinergic, dopaminergic, glutamatergic, and GABAergic dysregulation, which is rescued, at least partially, by psychiatric drugs (Grace, 2016; Murrough et al., 2017; Savitz, 2020). Psychedelic compounds have profound 

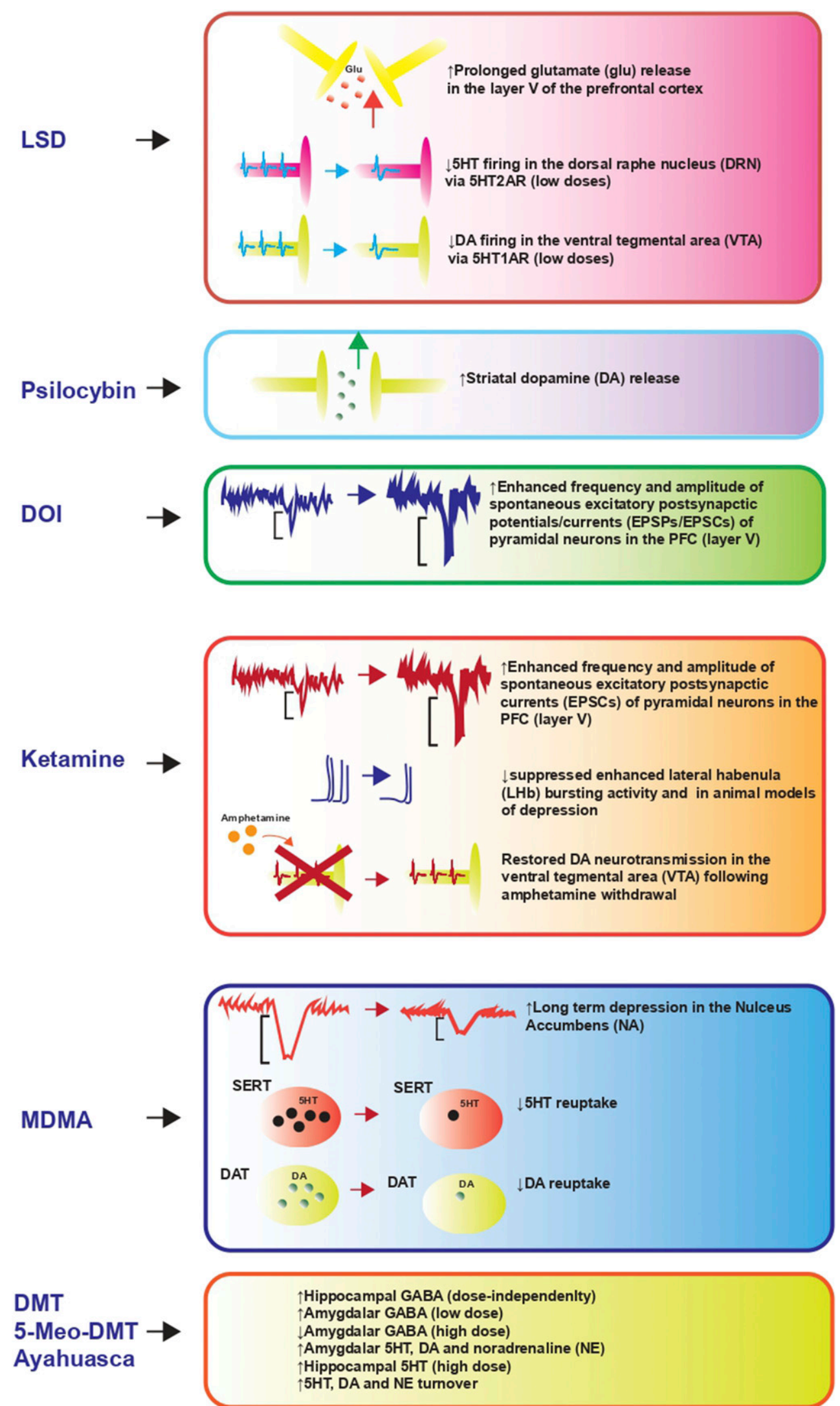

Fig. 3. Effects of psychedelic compounds on the serotonergic, dopaminergic, glutamatergic, and GABAergic neurotransmitter systems relevant to psychiatric disorders and comorbidities. For each compound, or group of compounds with similar pharmacology, the main known modulatory effects over the serotonergic, dopaminergic, glutamatergic, and GABAergic neurotransmitter systems in different areas of the brain are reported. 
modulatory outcomes on these neurotransmitter systems, which are of great relevance to psychiatry both in terms of understanding psychiatric pathophysiology and in terms of drug discovery and development opportunities (see Fig. 3).

\section{B. Serotonin}

Serotonin modulates an ample spectrum of physiological and pathological behaviors (Lesch et al., 1996; Domínguez-López et al., 2012). A common feature of psychiatric disorders is a chronic 5-HT depletion state, mediated at least partially by upregulated indoleamine 2,3-dioxygenase (IDO), the rate-limiting enzyme of tryptophan catabolism (Messaoud et al., 2019; Savitz, 2020). IDO represents a key node in the neuroimmune crosstalk, which decreases 5 -HT bioavailability and increases the toxic metabolic compound kynurenine, among others, exacerbating psychiatric symptoms (Messaoud et al., 2019; Savitz, 2020). Compounds that increase synaptic 5-HT availability have represented the core of psychiatric pharmacotherapy so far, and psychedelic compounds elicit profound effects over the serotonergic system, which could translate to long-term increased synaptic 5-HT availability.

1. Lysergic Acid Diethylamide. LSD is a $5-\mathrm{HT}_{2 \mathrm{~A}}$ receptor partial agonist (Marek and Aghajanian, 1996; Rickli et al., 2015; De Gregorio et al., 2016a). Similarly, LSD is an agonist at the $5-\mathrm{HT}_{1 \mathrm{~A}}$ receptor (Norman et al., 1985; Reissig et al., 2005; Rickli et al., 2016). Chronic administration of the widely used antidepressant and anxiolytic SSRI medications desensitizes the postsynaptic $5-\mathrm{HT}_{1 \mathrm{~A}}$ receptor, increasing serotonergic firing and ultimately eliciting antidepressant effects (Blier et al., 1993; Artigas et al., 1996; Haddjeri et al., 1998). The effects of LSD are strikingly similar to the mechanism of action of SSRIs in terms of 5- $\mathrm{HT}_{1 \mathrm{~A}}$ receptor dynamics. In fact, acutely, LSD decreases the firing and burst activity of $5-\mathrm{HT} / \mathrm{DRN}$ neurons via $5-\mathrm{HT}_{1 \mathrm{~A}}$ receptor activation, and this effect can be blocked by $5-\mathrm{HT}_{1 \mathrm{~A}}$ and $5-\mathrm{HT}_{2 \mathrm{~A}}$ receptor antagonism (De Gregorio et al., 2016b). On the contrary, repeated LSD administration increases firing and burst activity in the DRN via a mechanism mediated by $5-\mathrm{HT}_{1 \mathrm{~A}}, \mathrm{D} 2$, and TAAR receptors, resembling classic antidepressants (De Gregorio et al., 2016b). $\mathrm{LSD}$ is also an agonist at the $5-\mathrm{HT}_{2 \mathrm{~B}}$ receptor and a partial agonist at the $5-\mathrm{HT}_{2 \mathrm{C}}$ receptor (Fiorella et al., 1995; Egan et al., 1998; Rickli et al., 2016; Wacker et al., 2017). Interactions at the $5-\mathrm{HT}_{2 \mathrm{C}}$ receptor are thought to be responsible for the lack of addictive properties of psychedelic compounds (Canal and Murnane, 2017) and to partially mediate their anxiolytic and antidepressant effects (Millan, 2005; Nichols and Nichols, 2008). Agonist action has been described at the $5-\mathrm{HT}_{1 \mathrm{~B}}, 5-\mathrm{HT}_{1 \mathrm{D}}, 5-\mathrm{HT}_{1 \mathrm{E}}$, $5-\mathrm{HT}_{6}$, and 5- $\mathrm{HT}_{7}$ receptors (Hoyer, 1988; Erlander et al., 1993; Lovenberg et al., 1993; Blaho et al., 1997; Passie et al., 2008; Wacker et al., 2017). Evidence for LSD interaction at SERT remains controversial. In fact, although an in vitro study reported no interaction with SERT (Rickli et al., 2015), in vivo investigations reported decreased LSD effects in Sert null mice (Krall et al., 2008; Kyzar et al., 2016). Given that 1) suicidal patients with depression and PTSD have increased incidence of the low-expressing SERT polymorphisms (Austin et al., 2002; Oquendo et al., 2007), 2) long-term psychedelic users have increased SERT binding (Callaway et al., 1994), 3) intact SERT signaling is necessary for fear extinction (Young et al., 2017), and 4) there is lower incidence of psychological distress and suicidality among psychedelics users (Hendricks et al., 2015; Argento et al., 2017), it cannot be excluded that SERT modulation is a common pharmacological denominator mediating the therapeutic effects of psychedelics in psychiatric disorders. Aside from serotonergic interaction, the pharmacological and behavioral effects of LSD, and likely of other psychedelics, require activation of the $5-\mathrm{HT}_{2 \mathrm{~A}}-\mathrm{mGluR2}$ complex in glutamatergic pyramidal neurons (discussed below in the Biased Signaling, Biased Phosphoproteomics, and Psychedelic Compounds section) (Moreno et al., 2011). Recently, LSD was reported to increase the social adaptation to opinions similar to one's own through stimulation of $5-\mathrm{HT}_{2 \mathrm{~A}}$ receptors, which increase neuronal activity in the $\mathrm{mPFC}$ in response to social feedback processing (Duerler et al., 2020).

2. 3,4-Methylenedioxymethamphetamine. Upon ingestion, MDMA is rapidly absorbed in the intestinal tract (Kalant, 2001; Green et al., 2003). Peak plasmatic concentrations are reached after about 2 hours (Kalant, 2001). MDMA is metabolized by several pathways, such as $\mathrm{N}$-demethylation, $O$-dealkylation, conjugation, and $O$-sulfation, into 14 metabolites [reviewed in Green et al. (2003))]. Initially, $N$-demethylation gives rise to MDA, demethylenation gives rise to 3,4-dihydroxymethamphetamine and $N$-methyl- $\alpha$-methyldopamine, and ring hydroxylation gives rise to 6-HO-MDMA (Green et al., 2003). Given that both MDMA and its main metabolite, MDA, elicit psychoactive effects, and given that both interact (although to different extents) with the 5-HT system, it is difficult to separate the effects of one from the other. Therefore, the behavioral and neurobiological effects observed should be considered in light of this duality (Green et al., 2003).

MDMA is a $5-\mathrm{HT}_{2 \mathrm{~A}}$ receptor agonist, and this effect is thought to be responsible for the MDMA-induced mesolimbic DA release (Teitler et al., 1990; Orejarena et al., 2011). Acute MDMA administration induces a transient, MDA-mediated, dose-related increase in extracellular 5-HT in the mPFC, striatum, NAc, and hippocampus (Gough et al., 1991; Gudelsky and Nash, 1996; Kankaanpää et al., 1998; O'Loinsigh et al., 2001; Mechan et al., 2002), and repeated MDMA decreases 5HT concentration in the striatum (Nash and Yamamoto, 1992). MDMA is a weak $5-\mathrm{HT}_{1 \mathrm{~A}}$ receptor agonist, and MDMA administration results in a postsynaptic upregulation of this receptor in the cortex and hypothalamus 
that is noticeable 1 week after acute administration (Aguirre et al., 1998; Battaglia et al., 1988). Indirect 5$\mathrm{HT}_{1 \mathrm{~B}}$ receptor activation is involved in MDMA-induced hyperlocomotion when the substance is administered at low doses, whereas $5-\mathrm{HT}_{2 \mathrm{~A}}$ receptor activation is involved at high doses (Rempel et al., 1993; McCreary et al., 1999; Vollenweider et al., 2002). The acute MDMA-elicited 5-HT increase is disrupted by the administration of a 5-HT reuptake inhibitor, suggesting an inhibitory effect of MDMA on SERT (Gudelsky and Nash, 1996; Liechti et al., 2000; Mechan et al., 2002). Indeed, MDMA increases the available concentration of 5-HT via inhibiting SERT, reversing the direction of the membrane transporter and ultimately resulting in the accumulation of 5-HT in the synaptic cleft (Battaglia et al., 1988; Teitler et al., 1990; Koch and Galloway, 1997; Cole and Sumnall, 2003; Green et al., 2003; Verrico et al., 2007). MDMA use has been associated with decreased SERT density, and abstinence time and SERT density correlate positively, suggesting that these alterations are reversible to extents to be determined (Müller et al., 2019). The increase in available 5-HT after MDMA administration is only partially responsible for the enhanced release of DA given that the SSRI fluoxetine does not completely block the increase in 5-HT (Koch and Galloway, 1997). Preclinical studies corroborate the crucial involvement of SERT in the effects of MDMA given that MDMA has no psychostimulant effects in SERT ${ }^{-/-}$mice (Bengel et al., 1998). Remarkably, the effects of MDMA on SERT within the NAc are sufficient to elicit the prosocial effects of MDMA (Heifets et al., 2019).

The modulation of SERT by MDMA has implications in psychiatry given that SERT abundance has a dramatic impact on synaptic 5-HT and behavior. For example, genetic SERT variants and environmental exposure eliciting epigenetic modifications (Caspi et al., 2003) downregulate SERT expression, leading to increased psychiatric susceptibility (Karg et al., 2011; Lee et al., 2005). Accordingly, patients with MDD and BD, as well as suicide completers, have decreased SERT expression (Austin et al., 2002; Oquendo et al., 2007). On the other side of the spectrum, high-expressing SERT polymorphisms are associated with obsessive-compulsive disorder (Hu et al., 2006). Therefore, MDMA-induced SERT modulation could be exploited therapeuticallyfor example, in the treatment of ASD (Danforth et al., 2016, 2018). Future studies should assess acute and long-term MDMA-induced SERT transcriptional dynamics to clarify the effects of trauma, MDMA, and psychotherapy on SERT modulation.

MDMA has an inhibitory effect on the production of 5HT via decreasing tryptophan hydroxylase (the ratelimiting enzyme of 5-HT biosynthesis) activity within 15 minutes from administration (Johnson et al., 1992). This effect might be mediated by the peripheral generation of metabolites given that it is not appreciable in vitro (Schmidt et al., 1987; Che et al., 1995; Colado et al., 1999). The decrease in tryptophan hydroxylase has been observed for up to 2 weeks after a single acute administration of MDMA (Schmidt and Taylor, 1987). A further effect on the 5-HT system is exerted via the inhibitory effect of MDMA on monoamine oxidase A and monoamine oxidase $\mathrm{B}$, which are also involved in the extracellular monoaminergic increase after MDMA administration (Leonardi and Azmitia, 1994). Repeated MDA administration was shown to cause long-lasting depletion of cortical, hippocampal, and striatal 5-HT (Miller et al., 1997).

3. N,N-Dimethyltryptamine, 5-Methoxy-N,N-dimethyltryptamine, and Ayahuasca. The psychedelic tryptamine DMT, contained in the shrub Psychotria viridis (among other plants), is a $5-\mathrm{HT}_{2 \mathrm{~A} / 2 \mathrm{C}}$ receptor agonist, although its action at $5-\mathrm{HT}_{2 \mathrm{C}}$ receptors appears less relevant than its effects at $5-\mathrm{HT}_{2 \mathrm{~A}}$ receptors (Smith et al., 1998; Carbonaro et al., 2015). DMT stimulates 5HT release and inhibits its reuptake via interacting with SERT, in which it shows a high binding-to-uptake ratio, meaning that DMT interacts with different substrate and inhibitor SERT sites and that it might be actively taken up and stored by cells (Cozzi et al., 2009; Blough et al., 2014; Rickli et al., 2016). DMT is also both an inhibitor and substrate of the vesicular monoamine transporter 2 (VMAT2), which is involved in the reuptake of monoamines. Much like the effects at SERT, DMT also shows a high binding-to-uptake ratio, suggesting that VMAT2 might be another carrier involved in the uptake of DMT by cells (Cozzi et al., 2009). DMT also interacts with the $5-\mathrm{HT}_{1 \mathrm{~A}}, 5-\mathrm{HT}_{1 \mathrm{D}}, 5-\mathrm{HT}_{1 \mathrm{E}}, 5-\mathrm{HT}_{2 \mathrm{~B}}, 5-$ $\mathrm{HT}_{5 \mathrm{~A}}, 5-\mathrm{HT}_{6}$, and 5- $\mathrm{HT}_{7}$ receptors (Deliganis et al., 1991; Smith et al., 1998; Bunzow et al., 2001; Ray, 2010). The 5$\mathrm{HT}_{1 \mathrm{~A}}$ receptor antagonism potentiates the hallucinogenic effects of DMT, suggesting that $5-\mathrm{HT}_{1 \mathrm{~A}}$ receptor blockade can enhance $5-\mathrm{HT}_{2 \mathrm{~A}}$ receptor-mediated hallucinogenic effects (Strassman, 1996). Like other serotonergic psychedelics, DMT inhibits 5-HT firing in the DRN (Aghajanian et al., 1970).

The $\beta$-carbolines harmol, harmine, harmaline, and tetrahydroharmine are obtained from B. caapi (ayahuasca, or "vine of the souls") and function as MAOIs to block the metabolism of DMT, rendering it orally active (Pähkla et al., 2000; Yritia et al., 2002; Gambelunghe et al., 2008; Carbonaro et al., 2015). These compounds are tricyclic indole alkaloids that resemble tryptamines (Hamill et al., 2019). The presence of an endogenous counterpart in the pineal gland and retina, 6-methoxytetrahydro- $\beta$-carbolin (pinoline), has been reported (Langer et al., 1984; Leino, 1984). Harmine and harmaline act as reversible monoamine oxidase A inhibitors, whereas tetrahydroharmine binds $5-\mathrm{HT}_{2 \mathrm{~A}}$ and $5-\mathrm{HT}_{2 \mathrm{C}}$ receptors but not the presynaptic $5-\mathrm{HT}_{1 \mathrm{~A}}$ receptor as previously hypothesized (Udenfriend et al., 1958; Callaway et al., 1999). This effect results in an increase in available extracellular 5-HT and might be involved in 
the fast-onset antidepressant effects of ayahuasca in treatment-resistant MDD (Palhano-Fontes et al., 2019; Jiménez-Garrido et al., 2020) and potentially in the long-term neuromorphologic changes observed in longterm ayahuasca users (Bouso et al., 2015).

Although data on the acute monoaminergic effects of DMT and ayahuasca in humans are scarce, preclinical studies suggest potent and region-specific modulation of neurotransmitter release. For example, a study quantifying monoaminergic changes in the rat brain found that ayahuasca increases 5 -HT in the hippocampus at high doses and in the amygdala at all doses tested. Hippocampal 5-HT turnover was significantly decreased only at high doses. Amygdalar 5-HT turnover was decreased at all doses tested (de Castro-Neto et al., 2013). Similarly, a study investigating the effects of repeated ayahuasca administration on whole-brain neurotransmitter levels found an increase in wholebrain 5-HT levels in females receiving repeated ayahuasca at the highest does tested without significant changes in 5-HT turnover (Colaço et al., 2020). A study investigating potential anticancer activity of tryptamine and DMT found that these compounds inhibit IDO in vitro, potentiating antitumor activity (Tourino et al., 2013). Although this study was not psychiatryoriented, the fact that compounds that modulate the kynurenine pathway to decrease IDO activity are useful in psychiatry to decrease IDO emphasizes the relevance of this finding for psychiatry (Savitz, 2020). Consistent with the presence of naturally occurring MAOIs in ayahuasca, neurotransmitter turnover has been reported to be decreased upon ayahuasca administration in rodents (de Castro-Neto et al., 2013). Clinical imaging and biochemical studies should aim at closing the research gap on the monoaminergic effects of DMT and ayahuasca in humans to better elucidate its mechanism of action and maximize its therapeutic applications.

DMT is a selective SERT releaser (Blough et al., 2014; Rickli et al., 2016), whereas 5-MeO-DMT inhibits synaptosomal 5-HT reuptake (Berge et al., 1983). A study observed platelet SERT upregulation in humans after repeated ayahuasca ingestion, potentially as a result of repeated MAOI ingestion and/or repeated exposure to high levels of DMT, which affect SERT pharmacodynamics (Callaway et al., 1994; Blough et al., 2014). Tentatively, SERT polymorphisms could be investigated as predictors of positive/negative outcomes and experience intensity given that low- and high-expressing SERT genotypes have different psychopharmacological profiles (Pezawas et al., 2005; Möller et al., 2019). A close relative of DMT, 5-MeO-DMT, is found in several plants (such as A. peregrine) and in certain species of toads (such as $I$. alvarius, which also produces bufotenine) (Glennon and Rosecrans, 1982; Shen et al., 2010). 5-MeO-DMT acts as a $5-\mathrm{HT}_{2 \mathrm{~A}}, 5-\mathrm{HT}_{2 \mathrm{C}}, 5-\mathrm{HT}_{1 \mathrm{~A}}, \mathrm{~S} 1 \mathrm{R}$, and TAAR1 receptor agonist (Krebs-Thomson et al., 2006; Ray, 2010; Szabo et al., 2014). 5-MeO-DMT is a weak 5-HT uptake inhibitor (Blough et al., 2014). The affinity of 5-MeODMT for $5-\mathrm{HT}_{1 \mathrm{~A}}$ receptor is lower compared with that of DMT (McKenna et al., 1990).

4. Ketamine. Acute ketamine does not affect DRN/5HT firing (El Iskandrani et al., 2015) and the release of 5 -HT in the DRN (López-Gil et al., 2019), but it does enhance the stimulated release of 5-HT in the DRN (Tso et al., 2004). Moreover, ketamine enhances the release of 5-HT in the mPFC (López-Gil et al., 2019), strongly activating the 5-HT/PFC system through an AMPA receptor-independent mechanism (Ago et al., 2019). Supporting an enhancement of 5-HT release, 5-hydroxy-indoleacetic acid levels are increased after both acute and repeated ketamine administration (Lindefors et al., 1997). The antidepressant effects of ketamine appear to be elicited via a 5-HT-dependent mechanism given that 5-HT depletion in mice blocks its antidepressant effects (Gigliucci et al., 2013). Similarly to SSRIs, ketamine reduces 5-HT reuptake, although the exact mechanism underlying this effect have not been fully elucidated yet (Martin et al., 1982; Nishimura et al., 1998; Can et al., 2016; Yamamoto et al., 2013). Another known serotonergic interaction occurs at $5-\mathrm{HT}_{1 \mathrm{~B}}$ receptors (an action mediated by AMPA receptors) (Yamanaka et al., 2014), and this effect has been suggested to be involved in ketamine's antidepressant effects (du Jardin et al., 2018). Antagonist action has been reported at 5$\mathrm{HT}_{3}$ receptors at higher than clinically relevant concentrations (Appadu and Lambert, 1996). The analgesic action of ketamine is thought to be mediated by $5-\mathrm{HT}_{2 \mathrm{~B}}$ and $5-\mathrm{HT}_{2 \mathrm{C}}$ receptor activation, among others (Crisp et al., 1991). Increased 5-HT and NE efflux to the mPFC seems to be the essential step to produce ketamine's antidepressant effects, and such a process is thought to be mediated by ketamine-mediated glutamate release in the mPFC, which in turn excites DRN neurons both directly, via AMPA receptors on serotonergic terminals, and indirectly, via DRN efferents (Amargós-Bosch et al., 2006; Pham et al., 2017; López-Gil et al., 2019).

5. Psilocybin. Similarly to LSD, psilocybin decreases DRN/5-HT firing (Aghajanian and Hailgler, 1975). Serotonergic and dopaminergic activity are mutually regulated. Indeed, the selective lesion of DA neurons decreases the spontaneous firing activity of DRN/5-HT neurons by $60 \%$, whereas the selective lesion of $5-\mathrm{HT}$ neurons enhances the firing activity of VTA/DA neurons by $36 \%$, indicating an inhibitory effect of the $5-\mathrm{HT}$ input on DA neurons and an excitatory effect of DA on 5-HT neurons (Guiard et al., 2008; Dremencov et al., 2009). The 5-HT-mediated regulation of striatal DA neurotransmission via serotonergic afferents is involved in modulating reward, and serotonergic antidepressants improve mood, cognition, and hedonic behavior partially via 5-HT/DA interactions (Shuto et al., 2020). Employing radioligand binding assays, psilocybin has been demonstrated to display a high affinity at human serotonin receptors 5$\mathrm{HT}_{1 \mathrm{~A}}, 5-\mathrm{HT}_{2 \mathrm{~A}}$, and 5- $\mathrm{HT}_{2 \mathrm{~B}}$ (Chadeayne et al., 2020). 
$5-\mathrm{HT}_{2 \mathrm{~A}}$ receptors located on DA neurons within the striatum and NAc are thought to be involved in the modulation of psychedelic-induced, 5-HT-mediated dopaminergic effects (Vollenweider et al., 1999b). Psilocybin is thought to exert its psychedelic effects mostly through $5-\mathrm{HT}_{2 \mathrm{~A}}$ receptor agonism, occupying up to $72 \%$ of all human brain $5-\mathrm{HT}_{2 \mathrm{~A}}$ receptors at clinically relevant doses (Vollenweider et al., 1998; Winter et al., 2007; Quednow et al., 2012; Kometer et al., 2013; Madsen et al., 2019). Psilocybin also interacts with the $5-\mathrm{HT}_{1 \mathrm{~A}}$ receptor in vivo without producing behavioral effects and 5-HT $1 \mathrm{D}, 5-\mathrm{HT}_{1 \mathrm{E}}, 5-\mathrm{HT}_{2 \mathrm{~B}}, 5-\mathrm{HT}_{2 \mathrm{C}}, 5-\mathrm{HT}_{5}, 5-$ $\mathrm{HT}_{6}$, and $5-\mathrm{HT}_{7}$ receptors in vitro, although it is not known whether these interactions produce a clinically relevant effect (McKenna et al., 1990; Passie et al., 2002; Winter et al., 2007). Some authors have suggested that the $5-\mathrm{HT}_{1 \mathrm{~A}}$ receptor mediates the deficits in attentional performance induced by psilocybin (Carter et al., 2005). Although the modulatory action of psilocybin on mood states and emotional face recognition are mediated by the $5-\mathrm{HT}_{2 \mathrm{~A}}$ receptor, the psilocybin-induced bias toward the processing of positive emotions is mediated by other receptors, possibly the $5-\mathrm{HT}_{2 \mathrm{~B}}, 5-\mathrm{HT}_{2 \mathrm{C}}$, or $5-\mathrm{HT}_{1 \mathrm{~A}}$ receptor (Kometer et al., 2012). Also, psilocybin decreases 5-HT reuptake by inhibiting SERT (Rickli et al., 2016).

\section{Dopamine}

Although psychedelics are mainly associated with their action over the serotonergic system, important interactions (direct and/or indirect) exist with the dopaminergic system that are thought to mediate the psychotic-like effects of psychedelics and that might mediate at least some of the therapeutic (and also the side effects) of psychedelic compounds. Our laboratory has shown, for example, that although at low doses, LSD acts mostly on 5-HT firing activity, at doses higher than $30 \mu \mathrm{g} / \mathrm{kg}$, it also decrease the DA firing activity, effects that could explain why LSD can produce psychotic symptoms at higher doses (De Gregorio et al., 2016a). Several psychiatric symptoms are associated with DA effects. For example, anhedonia, one of the main dimensions of depression, associates with dysfunction of the DA system (Belujon and Grace, 2017). Similarly, in PTSD, genetic variability of DA receptors and stress-induced perturbations of DAT play a role in the development and severity of symptoms (Dragan and Oniszczenko, 2009; Drury et al., 2009). DAT dynamics are also altered in attention deficit hyperactivity disorder (Rowe et al., 1998).

1. Ketamine. The direct involvement of DA receptors in the pharmacology of ketamine is contradictory. In fact, although earlier studies reported high affinity and partial agonistic action at D2 receptors (Breier et al., 1998; Vollenweider et al., 2000; Kapur and Seeman, 2002; Seeman and Kapur, 2003) and ketamine-induced blockade of striatal DAT (Tsukada et al., 2001), later reports failed to replicate these findings, potentially because of the high doses necessary to initiate DA interactions (Aalto et al., 2002; Kegeles et al., 2002; Can et al., 2016). Nevertheless, ketamine is thought to restore DA activity and synaptic plasticity, at least partially, via D1 activation in the NAc (Belujon and Grace, 2014). Given that patients with MDD present NAc dysfunction with impaired dopamine-mediated reward circuits, a protective effect of ketamine on the DA system might relate to its therapeutic effects in patients with treatment-resistant depression (Feder et al., 2014; Kim et al., 2019; Krystal et al., 2019; Pizzagalli et al., 2009; Phillips et al., 2019). Acute ketamine induces a 5fold increase in DA release in the $\mathrm{mPFC}$ and enhances striatal DA release, whereas repeated (7 days) ketamine increases basal dopamine to almost double that of preketamine values and attenuates ketamine-induced DA release in the mPFC (Lindefors et al., 1997; Breier et al., 1998; El Iskandrani et al., 2015; Can et al., 2016). Moreover, ketamine antagonizes DA reuptake in the striatum (Tso et al., 2004). Although ketamine does not affect firing activity in the VTA (El Iskandrani et al., 2015), it restores the stress-induced dopaminergic dysfunction in the VTA (Rincón-Cortés and Grace, 2017). Nevertheless, ketamine restores the amphetamineinduced decrease of VTA activity (Belujon et al., 2016).

2. Lysergic Acid Diethylamide. LSD is a DA D1 (Schindler et al., 2012), D2 (Seeman et al., 2005), and D4 (Marona-Lewicka et al., 2009) receptor agonist. LSD modulates DA neurotransmission in a biphasic, dose-dependent fashion. Although at low doses no effects are observed in VTA/DA activity, the latter is decreased at higher doses via a multireceptorial mechanism involving $\mathrm{D} 2,5-\mathrm{HT}_{1 \mathrm{~A}}$, and TAAR1 receptors (Marona-Lewicka et al., 2005; De Gregorio et al., 2016b). Confirming a dopaminergic involvement in LSD pharmacology, in vitro studies confirmed that LSD binds the human and murine D1 and D2 receptors (Pieri et al., 1974; Watts et al., 1995; Marona-Lewicka et al., 2009; Rickli et al., 2015). Moreover, the D4 receptor is involved in the discriminative stimulus effects in rats of LSD (MaronaLewicka et al., 2009).

3. 3,4-Methylenedioxymethamphetamine. MDMA stimulates dopaminergic neurotransmission via 1) reversing the direction of the DA transporter; 2) a $5-\mathrm{HT}_{2 \mathrm{~A}}$ receptor-induced DA release, which results in an overall increase in synaptic DA availability; and 3) an inhibition of DA nigrostriatal and mesolimbocortical reuptake in a time-, dose-, and region-dependent manner (Yamamoto and Spanos, 1988; Gudelsky et al., 1994; Bankson and Cunningham, 2001; Mayerhofer et al., 2001; Verrico et al., 2007). Acute MDMA induces DA release, and repeated administration decreases DA concentration in the striatum (Nash and Yamamoto, 1992). At high doses (i.e., $10 \mathrm{mg} / \mathrm{kg}$ ), the effects of MDMA on DA release are more marked in the caudate nucleus $(\sim 400 \%)$ compared with the NAc $(\sim 200 \%)$, whereas at lower doses (i.e., 2.5 and $5 \mathrm{mg} / \mathrm{kg}$ ), this 
increase is almost equivalent in the two regions and is not so remarkable $(\sim 20 \%)$ (Yamamoto and Spanos, 1988). Interaction of MDMA with the DA transporter is relatively low, and it is thought to be partially responsible for the increase in extracellular DA after MDMA administration (Hagino et al., 2011). Decreased DAT binding in the striatum has been reported in rodents 1 week after acute MDMA (Biezonski et al., 2013). In humans, a decrease in striatal DAT was observed only in recreational MDMA users who also used amphetamines (Reneman et al., 2002a). Lastly, MDMA possesses weak D1 and D2 receptor affinity (Battaglia et al., 1988), and these effects are involved in MDMA-induced hyperlocomotion (Risbrough et al., 2006), while D2 receptors are involved in the dopaminergic toxicity induced by high doses of MDMA (Granado et al., 2011).

The MDMA-induced increase in available 5-HT is partially responsible for the increased release of DA given that fluoxetine (SSRI) only partially blocks the MDMA-induced increase in extracellular DA (Koch and Galloway, 1997). These dopaminergic effects elicited by MDMA are the basis for concern around potential abuse liability and addiction. However, at clinically relevant doses (i.e., $1.5 \mathrm{mg} / \mathrm{kg}$ ), the increase in DA release is minimal, suggesting that abuse concerns are not so relevant at clinical doses (Yamamoto and Spanos, 1988; Pantoni and Anagnostaras, 2019). As discussed earlier, the hepatic metabolism of MDMA to MDA and other catechols with substantial DA activity is likely involved in the potential neurotoxic effects of MDMA (Green et al., 2003).

4. $N, N$-Dimethyltryptamine, 5-Methoxy- $N, N$-dimethyltryptamine, and Ayahuasca. Acute Ayahuasca administration increases amygdalar DA levels and decreases its turnover (de Castro-Neto et al., 2013). A recent study reported that repeated ayahuasca administration increases the concentration of the DA metabolites 3,4-dihydroxyphenylacetic acid in whole-brain homogenates in rodents (Colaço et al., 2020). Earlier studies reported that DMT increases striatal (Smith, 1977) and whole-brain DA synthesis (Waldmeier and Maitre, 1977), although it lacks direct dopaminergic activity (Hungen et al., 1975). DMT was also reported to increase the levels of the extraneuronal DA metabolite 3-methoxytyramine (Waldmeier et al., 1976) acutely and after 1 month of treatment. This suggests that repeated DMT might accelerate DA turnover, potentially as a mechanism to compensate the increased DA synthesis (Waldmeier et al., 1976; Smith, 1977; Colaço et al., 2020). One study reported decreased levels of forebrain DA in response to DMT (Haubrich and Wang, 1977). Given that S1R agonists modulate DA neurotransmission, important effects of ayahuasca, DMT, and 5-MeO-DMT over the dopaminergic system are plausible, which might be involved in the amelioration of suicidality and addiction behaviors by modulating reward-related circuitries. For example, S1R ligands can interact with the DA transporter, preventing DA efflux and nullifying drug-induced reward (Sambo et al., 2017, 2018). Therefore, future studies should investigate whether these compounds could address reward circuitry dysfunction in addiction (Sambo et al., 2017).

Together, a dose-dependent involvement of the DA system is recognized in the action of psychedelics and entactogens. Given the role of the DA system in mediating reward responses and developing addiction, future studies are required to clarify whether 1) acute and repeated psychedelic administration leads to changes in homeostasis of reward-related pathways 2) and, if so, at which doses; 3) whether this represents a concrete danger for abuse; and 4) whether adjunctive therapies can pharmacologically counteract these effects without blocking the desirable effects.

\section{Glutamate}

Glutamate is involved in excitatory neurotransmission, synaptogenesis, synaptic plasticity, memory, mood, and cognition (Murrough et al., 2017). Stress responses are partly glutamate-dependent (Nasca et al., 2015; Moghaddam and Javitt, 2011), and enhanced glutamatergic neurotransmission during stress is beneficial for returning to homeostasis. Glutamate can act via NMDA receptors, AMPA receptors, or metabotropic receptors. Aside from its physiological role in stress responses (Sanacora et al., 2008), dysfunction of the glutamatergic ionotropic receptor NMDA can be triggered by repeated stress and is appreciable in populations with depression (Marsden, 2011). NMDA receptor modulation is part of both the mechanism of action and outcome of antidepressant therapies (Trullas and Skolnick, 1990; Layer et al., 1995; Baumann, 1996; Sanacora et al., 2008; Bennett, 2010; Autry et al., 2011).

Glutamate release plays a key role in mediating the acute effects of psychedelics, and it is likely involved in the creation of a long-lasting state of synaptic and neural plasticity in corticolimbic circuits, which might represent the substrate for clinical improvement. The glutamatergic system drives the frontal hypermetabolic state induced by psychedelics, which also correlates to therapeutic efficacy and enhanced neurotrophic activity (Aghajanian and Marek, 1999; Scruggs et al., 2003; Muschamp et al., 2004; Lambe and Aghajanian, 2006; Carhart-Harris et al., 2016c).

NMDA receptor is involved in neuroplasticity, and corticolimbic NMDA receptor hypofunctioning is thought to be involved in the pathogenesis of SCZ (Jentsch and Roth, 1999; Olney et al., 1999). AMPA receptors are also involved with stress, depression, antidepressant treatment, and neurogenesis (Alt et al., 2005, 2006a,b; Chourbaji et al., 2008; Kallarackal et al., 2013).

1. Lysergic Acid Diethylamide. LSD elevates prefrontal-limbic glutamate via stimulating postsynaptic $5-\mathrm{HT}_{2 \mathrm{~A}}$ receptors on pyramidal cells in deep cortical layers (Aghajanian and Marek, 1997, 1999; Scruggs et al., 2003; Muschamp et al., 2004), and this effect is 
reversed by $5-\mathrm{HT}_{2 \mathrm{~A}}$ receptor antagonists (Vollenweider et al., 1998), AMPA receptor antagonists (Benneyworth et al., 2007), selective antagonists of the NR2B subunit of NMDA receptors (Zhang and Marek, 2008), and positive mGluR2 allosteric modulators (Lambe and Aghajanian, 2006). Electrophysiological studies show that antagonism of the NMDA receptor subunit NR2B suppresses the glutamate release induced by LSD, suggesting an essential role for glutamate in the downstream effects of LSD (Lambe and Aghajanian, 2006). Accordingly, neurodegeneration, aging, and inflammation associate with layer 5 synaptic and dendritic dysfunction (de Brabander et al., 1998; Fogarty et al., 2015). LSD also needs a functional mGluR2 or mGluR3 given that mGluR2/3 antagonists block LSDinduced gene transcriptional changes (Moreno et al., 2013). Therefore, it seems plausible that psychedelics might have protective effects on the neurodegenerative aspect of MDD and other psychiatric disorders via their glutamatergic-enhancing effects in corticolimbic areas.

2. Psilocybin. Multimodal neuroimaging studies after psilocybin administration reported a regionspecific hypermetabolic state, especially in the frontolateral and frontomedial cortices. Given that these areas signal primarily via glutamatergic projections and are rich in 5- $\mathrm{HT}_{2 \mathrm{~A}}$-mGluR2 heteroreceptors, it is possible that glutamate might mediate these effects (González-Maeso et al., 2008; Carhart-Harris et al., 2016c). Interestingly, in a preclinical study, although administration of psilocybin or ketamine alone did not ameliorate depressive-like behavior, the concomitant administration of these compounds elicited antidepressantlike effects comparable to those of fluoxetine (MartinRuiz et al., 2001). This finding suggests that subthreshold doses of multiple psychedelic compounds might have synergistic antidepressant effects, potentially via activating pathways that converge on the modulation of corticolimbic serotonergic and glutamatergic systems (Martin-Ruiz et al., 2001).

3. Ketamine. The main effects of ketamine and its metabolite norketamine were first attributed to NMDA receptor antagonism (Lodge et al., 1982; Anis et al., 1983; MacDonald et al., 1987; Franks and Lieb, 1994; Ebert et al., 1997). Subsequent work found that mTOR activation is a step required for ketamine to elicit antidepressant effects and to enhance synaptogenesis in the PFC ( $\mathrm{Li}$ et al., 2010). It seems likely that these effects are not mutually exclusive and that the enhancement of AMPA receptors over NMDA receptor throughput in cortical circuits, leading to increased plasticity and neurogenesis, might be responsible for the onset of antidepressant effects (Maeng et al., 2008). The antidepressant effects of ketamine are elicited via NMDA receptor antagonism, which results in an increase of AMPA receptor activation compared with NMDA receptor activation (Maeng et al., 2008; Sanacora et al., 2008; Zarate and Manji, 2008; Autry et al., 2011).
Ketamine rapidly increases extracellular cortical and striatal glutamate (Stone et al., 2012; López-Gil et al., 2019), enhancing cortical excitability (Cornwell et al., 2012), and this action correlates with improvement of psychiatric symptoms (Stone et al., 2012; Abdallah et al., 2015; Lisek et al., 2017). Moreover, ketamine increases glutamate release in the DRN (López-Gil et al., 2019). The metabolism of ketamine to hydroxynorketamine may be another key necessary step to elicit antidepressant effects, which are mediated by early and sustained activation of AMPA receptor, independently from NMDA receptor (Maeng et al., 2008). The therapeutic effects of ketamine may also be driven, at least partially, by a downregulation of excitatory amino acid transporter (EAAT) 2, which delays glutamate reuptake (Lisek et al., 2017). Ketamine administration 1) potentiates firing but decreases burst activity in glutamatergic neurons in the PFC (Jackson et al., 2004), 2) decreases firing activity in PFC GABAergic interneurons (ultimately diminishing the inhibitory influence on the cortex) (Homayoun and Moghaddam, 2007), 3) enhances striatal dopamine release (Breier et al., 1998), and 4) modulates reticular thalamus (RT) activity (Troyano-Rodriguez et al., 2014). These effects resemble the schizophrenic brain and can be partly restored by antipsychotic treatment (Kargieman et al., 2007). AMPA receptor blockade prevents the behavioral effects of ketamine and the increase in firing and bursting in the locus coeruleus and VTA activity elicited by ketamine (Moghaddam et al., 1997; El Iskandrani et al., 2015). Moreover, AMPA receptor potentiation of CA3 pyramidal neurons was also observed after ketamine (El Iskandrani et al., 2015). These findings suggest that an enhancement of catecholaminergic neurotransmission via AMPA receptor-mediated neurotransmission is required for ketamine to elicit antidepressant effects (Maeng et al., 2008; Sanacora et al., 2008; El Iskandrani et al., 2015). Importantly, ketamine blocks NMDA receptor-mediated burst firing in the lateral habenula, the "antireward" center, disinhibiting monoaminergic reward centers and rapidly relieving depression (Yang et al., 2018). A novel mechanism has recently been suggested that might at least partially explain the psychotomimetic and/or the antidepressant effects of ketamine: although physiologically $\alpha_{2}$-adrenoceptors and $\mathrm{GABA}_{\mathrm{B}}$ receptors selectively inhibit AMPA and NMDA receptors, respectively, ketamine reduces the levels and activity of the neuromodulatory regulator of $\mathrm{G}$ protein signaling 4, leading to selectivity loss and broad glutamatergic inhibition (Lur et al., 2019).

4. 3,4-Methylenedioxymethamphetamine. Acute MDMA increases glutamate release in the anteromedial striatum and dorsal hippocampus (Nash and Yamamoto, 1992; Anneken et al., 2013), whereas repeated MDMA does not affect glutamate efflux in the striatum (Nash and Yamamoto, 1992). This effect might be mediated by the stimulation of $5-\mathrm{HT}_{2 \mathrm{~A} / 2 \mathrm{C}}$ receptors on non-neuronal 
cells given that pretreatment with the $5-\mathrm{HT}_{2 \mathrm{~A} / 2 \mathrm{C}}$ receptor antagonist ketanserin prevents the increase in hippocampal glutamate release (Anneken and Gudelsky, 2012). Microiontophoretic investigations found that MDMA inhibits neuronal firing in the NAc similarly to 5-HT and DA, and this effect might be due to the MDMAinduced increase in 5-HT and DA release (White et al., 1994, 1995).

Repeated administration of MDMA during adolescence leads to important neuroadaptive changes in glutamatergic-related gene expression in corticolimbic structures (Kindlundh-Hogberg et al., 2008). For example, GluR2, mGluR1, mGluR5, NR1, NR2A, NR2B, EAAT1, and EAAT2 were increased in the adult cortex, whereas GluR3, NR2A, and NR2B receptor subunits were increased in the caudate putamen after repeated adolescent MDMA (Kindlundh-Hogberg et al., 2008). Moreover, transcription levels of GluR1 were reduced in the hippocampus, whereas GluR1, GluR3, mGluR1, and mGluR3 were increased in the hypothalamus (KindlundhHogberg et al., 2008). These effects might be involved in the neurotoxic effects of MDMA binge use and repeated use in individuals of young age and potentially in the MDMAinduced, 5-HT-mediated loss of parvalbumin interneurons in the hippocampal dentate gyrus (Collins et al., 2015). NMDA receptors take part in the acquisition of conditioned rewarding effects of MDMA (García-Pardo et al., 2015), whereas NMDA and AMPA receptor antagonists block these, as well as striatal DA efflux and GABAergic toxicity in the hippocampus, suggesting potential in the treatment of MDMA and methamphetamine abuse (Finnegan and Taraska, 1996; Johnson and Kotermanski, 2006; Huff et al., 2016; García-Pardo et al., 2018, 2019).

5. N,N-Dimethyltryptamine, Ayahuasca, 5-Methoxy$N, N$-dimethyltryptamine. Acute ayahuasca administration (up to $800 \mathrm{mg} / \mathrm{kg}$ ) did not affect glutamate levels in the hippocampus or amygdala in rats (de Castro-Neto et al., 2013). mGluR5 is downregulated by $5-\mathrm{MeO}-\mathrm{DMT}$ in brain organoids, and this receptor is involved in addiction; therefore, the lower incidence of alcohol and substance use disorder among people who ingest psychedelics multiple times during their lifetime could be mediated by the influence of this receptor (Bird et al., 2008; Stoker et al., 2012; Dakic et al., 2017). Future studies should investigate the glutamatergic effects elicited by repeated administration of ayahuasca, DMT, and 5-MeO-DMT, which might mediate the activation of antidepressant and antiaddiction corticolimbic circuits (Bouso et al., 2013; Riga et al., 2014; Osório et al., 2015; Sanches et al., 2016; Morales-Garcia et al., 2017; Inserra, 2018; da Silva et al., 2019; de Almeida et al., 2019; Palhano-Fontes et al., 2019; Zeifman et al., 2019; Murphy-Beiner and Soar, 2020).

\section{E. $G A B A$}

GABA is the main inhibitory neurotransmitter in the adult brain, and GABA-orchestrated circuits play key roles in physiological processes such as pain modulation (Francois et al., 2017) and in the serotonergic modulation of cortical networks (Cathala et al., 2019). GABAergic dysfunction is appreciable across psychiatric disorders (Petty, 1995; Vaiva et al., 2006). For example, NMDA receptor hypofunction in corticolimbic GABAergic interneurons, leading to corticolimbic pyramidal neuron disinhibition, is a putative pathologic mechanism of schizophrenia (Olney et al., 1999; Belforte et al., 2010; Coyle, 2012). In response to stress exposure, a downregulation of $\mathrm{GABA}_{\mathrm{B}}$ receptors has been described, which is reversed in antidepressant responders (Lloyd et al., 1985; Martin et al., 1989). In depression, altered cortical GABAergic neurotransmission disrupts canonical prefrontal information processing (Choudary et al., 2005). Accordingly, patients with depression have decreased levels of cerebrospinal GABA (Kasa et al., 1982), and antidepressants re-establish cortical GABA equilibria (Sanacora et al., 2002). Although most studies investigating the neurotransmitter effects of psychedelics focused largely on the serotonergic, glutamatergic, and dopaminergic systems, the GABAergic system is also involved in the acute and potentially the long-term effects of psychedelic compounds.

1. Ketamine. The main body of work investigating the effects of psychedelics on the GABAergic system probably comes from studying the effects of ketamine on GABAergic corticolimbic interneurons (Behrens et al., 2007; Quirk et al., 2009; Belforte et al., 2010). The effects of ketamine in modulating GABAergic populations within the CSTC circuit have been described (Behrens et al., 2007; Höflich et al., 2015; Rivolta et al., 2015; Jeevakumar and Kroener, 2016), including a disruption of RT activity (Liu et al., 2016; Mahdavi et al., 2020). The antidepressant effects of ketamine require the modulation of $\mathrm{GABA}_{\mathrm{A}}$ and $\mathrm{GABA}_{\mathrm{B}}$ receptors in discrete networks of cortical PV+ GABAergic interneurons (Quirk et al., 2009; Rosa et al., 2016; Yang et al., 2016; Wang et al., 2017a; Gerhard et al., 2020) and a downregulation of GABA reuptake (Mantz et al., 1995; Flood and Krasowski, 2000). Further strengthening the case for a modulatory effect of ketamine on the GABAergic system, ketamine administration in young animals leads to alterations in adult GABA circuits (Jeevakumar and Kroener, 2016). Both corticothalamic (Dawson et al., 2013; Anderson et al., 2017) and thalamocortical (Höflich et al., 2015) neurotransmission is modulated by ketamine via NMDA receptor blockade on GABAergic neurons in the RT, which leads to a disinhibition of DA neurons and increased DA release (Liu et al., 2016). Such effects on the RT are of particular interest given that NMDA receptor antagonism in the RT disrupts T-type calcium channel-mediated bursting in GABAergic neurons, leading to decreases in burst activity (Zhang et al., 2009) that are reminiscent of SCZ (Ferrarelli and Tononi, 2011; Hunt et al., 2017). Given that the RT (Min, 2010) and, more broadly, the CSTC circuit 
(Herrera et al., 2016) are 1) implicated in the generation of consciousness (Ward, 2011), 2) dysfunctional in psychiatric conditions (Swerdlow and Koob, 1987; Brown et al., 2017a; Posner et al., 2017), and 3) targeted by psychedelics (Scruggs et al., 2000; Preller et al., 2019), it is plausible that a modulatory action of this circuit is at the therapeutic core of psychedelics in psychiatric disorders (discussed in the Going Beyond Receptors: Neuronal Circuits Activated by Psychedelic Drugs section).

2. Lysergic Acid Diethylamide. LSD and DOI are partial agonists at $5-\mathrm{HT}_{2 \mathrm{~A}}$ receptors in cortical interneurons, and this effect is involved in the inhibition of specific cortical pyramidal networks (Marek and Aghajanian, 1996). DOI was shown to activate cortical GABAergic interneurons and to dose-dependently increase cortical GABA levels (Abi-Saab et al., 1999; Wischhof and Koch, 2012). DOI directly depolarizes interneurons (Marek and Aghajanian, 1994), and this effect might be mediated by direct presynaptic 5-HTR binding by DOI or by psychedelic-induced, 5 -HT-mediated presynaptic stimulation (Willins et al., 1997; Jakab and Goldman-Rakic, 1998). Moreover, DOI upregulates PV expression in cFOS+ GABAergic interneurons in the $\mathrm{mPFC}$ and somatosensory cortex (Martin and Nichols, 2016).

3. 3,4-Methylenedioxymethamphetamine. MDMA-induced DA release is mediated at least partially by GABAergic interactions. For example, MDMA enhances GABA efflux in the VTA, which dampens the MDMA-mediated DA release in the NAc shell (Bankson and Yamamoto, 2004) and decreases GABA efflux in the substantia nigra (Yamamoto et al., 1995). MDMA also modulates GABArelated gene expression in a specific spatiotemporal fashion. For example, MDMA increased GABA transporter (GAT) 1 and GAT4, but not GAT2 transcription, in the PFC and midbrain (Peng and Simantov, 2003). These results suggest a pivotal effect of MDMA on GABAergic gene expression (Simantov and Peng, 2004). Accordingly, a binge-like MDMA administration decreased PV+ GABAergic interneurons in the dorsal hippocampus (Anneken et al., 2013). Interestingly, repeated exposure to MDMA decreases the number of PV+ GABA neurons in the dentate gyrus, and this effect is attenuated by pretreatment with the anti-inflammatory ketoprofen, suggesting that the cyclooxygenase-mediated pathway might be involved (Anneken et al., 2013).

4. Psilocybin. 5- $\mathrm{HT}_{2 \mathrm{~A}}$ receptors located on GABAergic interneurons within the striatum and NAc might be involved in the modulation of psychedelic-induced, 5HT-mediated dopaminergic effects (Vollenweider et al., 1999b). Given that psilocybin, and psychedelics in general, decrease neuronal activity in discrete brain areas, such as the default mode network (DMN) (Carhart-Harris et al., 2012, 2016c; Palhano-Fontes et al., 2015; Tagliazucchi et al., 2016), these effects could be mediated by the serotonergic action of psilocybin or psilocybin-induced 5-HT release on GABAergic interneurons in these areas, enhancing local inhibition, as is the case for DOI (Cumming-Hood et al., 1993; Marek and Aghajanian, 1996) and LSD (Marek and Aghajanian, 1996).

5. N,N-Dimethyltryptamine, 5-Methoxy-N,N-dimethyltryptamine, Ayahuasca. Ayahuasca increases GABA levels in the hippocampus and in the amygdala only at high doses in preclinical models (de Castro-Neto et al., 2013). This mechanism could be involved in the retrieval of repressed traumatic memories experienced by some individuals who ingest ayahuasca given that the GABAergic network synchronization in the hippocampus can create the prerequisites for the synaptic changes (Paulsen and Moser, 1998; Inserra, 2018) required for memory reconsolidation (Tronson and Taylor, 2007). Such effects could be also exploited for the treatment of substance abuse (Liester and Prickett, 2012; Thomas et al., 2013) given the high comorbidity rates with PTSD (Brown and Wolfe, 1994).

\section{F. Norepinephrine}

Although not often discussed in relation to psychedelics, dissociative anesthetics, and entheogens, the neuromodulatory NE system might play an important role in both the acute effects of these compounds and in the longterm therapeutic improvements observed (Aston-Jones and Cohen, 2005). NE is the main neurotransmitter of the autonomic nervous system, which regulates crucial functions such as cognition, emotions, stress responses (including fight or flight), learning, memory, behavioral flexibility, sleep/wake cycle, heart rate, digestion, respiration, and sexual arousal (Aston-Jones and Bloom, 1981; Aston-Jones et al., 1999; Berridge and Waterhouse, 2003; Sara, 2009; Kreibig, 2010; Howells et al., 2012). NE plays a fundamental role in the stress response. In situations of stress, CRF is secreted by the paraventricular nucleus of the hypothalamus, and it activates NE neurons in the locus coeruleus (LC), which in turn stimulate further CRF production, leading to HPA axis activation and, potentially, HPA dysfunction (Valentino et al., 1983; Dunn and Swiergiel, 2008). Interestingly, the female LC/NE system appears to be more sensitive to CRF overexpression (and therefore more susceptible to stress) because of the estrogen-enhanced $\mathrm{NE}$ release in target brain areas (Bangasser et al., 2013, 2016).

The LC is the brain area that contains the majority of NE neurons and plays an important role in determining stimuli salience and optimizing performance via modulating the activity of frontal areas such as the orbitofrontal cortex and ACC (Aston-Jones and Bloom, 1981; Aston-Jones and Cohen, 2005; Sara, 2009). NE binds adrenergic receptors, which are both autoreceptors and heteroreceptors: $\alpha$ and $\beta$, both of which have at least two subtypes (De Blasi, 1990; Fraser and Venter, 1990; Strosberg, 1993, 1995). The $\alpha 2$ receptors are modulated by chronic, unescapable stress (Taneja et al., 2011), and drugs that modulate noradrenergic receptors are used 
for the treatment of PTSD (Southwick et al., 1999). Dysfunction of the NE system is appreciable in stressinduced psychiatric disorders (Klimek et al., 1997; Kosten et al., 1987). Some studies found that CSF NE levels are increased in patients with depression (Wong et al., 2000), whereas others suggested that NE turnover might be increased in depression given that patients with depression have increased levels of the NE metabolite 3-methoxy,4-hydroxyphenylethylene glycol (Koslow et al., 1983). The blockage of the reuptake of the NE by the tricyclics or the serotoninnorepinephrine reuptake inhibitors has antidepressant effects (Gobbi and Blier, 2005).

1. Ketamine. The dissociative anesthetics ketamine and PCP have been shown to increase NE release and to inhibit its reuptake. Ketamine increases NE efflux in the mPFC in vivo (Kubota et al., 1999; López-Gil et al., 2019 and NE efflux in the ventral bed nucleus of the stria terminalis in vitro (Tso et al., 2004). No changes in the DRN efflux of NE have been reported (López-Gil et al., 2019). Early studies suggested that ketamine potentiates the contraction-inducing effects of $\mathrm{NE}$ on vascular adrenergic neurons (Nedergaard, 1973). $\alpha$-Adrenergic, but not $\beta$-adrenergic, blockade attenuated most of the cardiorespiratory responses to ketamine, suggesting an involvement of $\alpha$-adrenergic neurotransmission in the effects of ketamine (Traber et al., 1970, 1971). More recent studies reported that ketamine interacts both with peripheral $\alpha_{1^{-}}$and $\beta_{2^{-}}$ adrenoceptor binding sites, eliciting vasoconstriction and vasodilation, respectively (Bevan et al., 1997). Supporting an involvement of $\alpha_{2}$-adrenoceptors in the mechanism of action of ketamine, $\alpha_{2}$-adrenoceptor antagonism attenuates the cardiostimulatory and psychotomimetic effects of ketamine anesthesia (Doak and Duke, 1993; Tanaka and Nishikawa, 1994; Levanen et al., 1995). Further, ketamine attenuates the cardiodepressant effects of the $\alpha_{2}$-adrenoceptor agonist dexmedetomidine (Char et al., 2013).

Ketamine and PCP were shown to inhibit NE reuptake in NE neurons of the rat cortex (Taube et al., 1975; Mandela and Ordway, 2006). However, although the inhibitory activity of PCP was high (to extents similar to those of cocaine), the inhibitory activity of ketamine was much lower (Taube et al., 1975). Similarly, in the bed nucleus of the stria terminalis, ketamine decreased the rate of NE reuptake (Tso et al., 2004). Other studies reported that ketamine inhibits $\mathrm{NE}$ reuptake in the heart tissue (Miletich et al., 1973; Aronson and Hanno, 1978; Salt et al., 1979) and that the antagonistic effects of ketamine at NET are responsible for the arrhythmias generated by the drug at high doses (Koehntop et al., 1977). However, different timing of ketamine exposure appears to have different effects over NE reuptake. For example, acute (30 minutes) exposure of bovine adrenal medullary cells to ketamine decreases NE uptake (Hara et al., 1998), whereas exposure for longer than 3 hours increases it (Hara et al., 2000). Importantly, 24-hour incubation with ketamine increases NET mRNA (Hara et al., 2000, 2002). The exact mechanisms through which ketamine affects NET remain unknown. It has been hypothesized that the inhibitory effects of ketamine on NE reuptake might be mediated by a direct action at the desipramine binding site of NET (Hara et al., 1998) or by an indirect effect of NMDA receptors over NET (Mandela and Ordway, 2006).

\section{2. \\ 3,4-Methylenedioxymethamphetamine.} MDMA is an $\alpha 2$-adrenergic receptor agonist (Battaglia et al., 1988; Bexis and Docherty, 2005, 2009; Hysek et al., 2012a, 2013). Lower affinity has been described for $\beta$-adrenoceptors (Battaglia et al., 1988). Agonism at $\beta 3$-adrenergic receptor, together with agonism at $\alpha 1$ adrenergic receptor, is thought to be involved in hyperthermia-induced rhabdomyolysis given that concomitant antagonism at $\beta 3$ - and $\alpha 1$-adrenergic receptor attenuates hyperthermia-induced rhabdomyolysis (Sprague et al., 2004). The empathogen MDMA increases NE release (Rothman et al., 2001; Verrico et al., 2007; Mithoefer et al., 2018). Moreover, MDMA interacts with NET, inhibiting NE reuptake (Rothman et al., 2001; Verrico et al., 2007). Accordingly, the NET inhibitors reboxetine and desipramine, respectively, decrease the biological (such as plasmatic NE release and cardiostimulant action) and subjective (such as subjective drug high, psychostimulation, and emotional excitation) effects of MDMA in humans (Hysek et al., 2011) and the acute MDMA-induced goal-oriented task impairments observed in other primates (Verrico et al., 2008). Further suggesting an adrenergic involvement in the biological and cardiostimulatory effects of MDMA, the $\beta$-adrenergic antagonist propranolol, but not the $\alpha$-adrenergic antagonist prazosin, suppresses the MDMA-induced glycogenolysis and consequent glucose release in the striatum (Pachmerhiwala et al., 2010), whereas the nonselective $\beta$-blocker pindolol and the $\alpha 1$ - and $\beta$-adrenergic antagonist carvedilol attenuate the cardiostimulatory effects of MDMA (Hysek et al., 2010, 2012a,b).

A study investigating a potential causal role of genetic variants at the NET gene, which could explain biological and subjective variability in the effects of MDMA, found a weak correlation between NET polymorphisms and the cardiostimulatory effects of MDMA (Vizeli et al., 2018). Specifically, 1) individuals carrying the GG genotype of the NET polymorphism rs1861647 presented greater cardiostimulant effects compared with subjects with one or no $G$ alleles, 2) subjects carrying a C allele of the NET polymorphism rs2242446 presented greater MDMA-induced heart rate elevations compared with the TT genotype, and 3) G allele carriers of the polymorphism rs36029 displayed decreased elevations of arterial pressure compared with the AA genotype (Vizeli et al., 2018). Lastly, NE is involved in the MDMA-induced prolongation of the latency and 
light-induced miosis (pupil constriction) (Hysek and Liechti, 2012).

3. Lysergic Acid Diethylamide. LSD is an $\alpha 2$-adrenoceptor agonist (Marona-Lewicka and Nichols, 1995). Microiontophoretically applied LSD was reported to slightly increase the firing rate of some, but not all, LC/NE neurons, whereas intravenous LSD induces a delayed partial suppression of some others (Svensson et al., 1975; Rogawski and Aghajanian, 1979). Interestingly, aside from its direct effects on firing rate, LSD increases the reactivity of LC/NE neurons to stimuli that normally do not activate these neurons during anesthesia, such as fur stroking or air puffs, and it potentiates the response to stimuli that normally excite LC/NE neurons, such as skin pinching (Aghajanian, 1980). Further, LSD was reported to decrease the NE-induced cAMP increase in vivo in the rat hypothalamus and brainstem and in vitro in the hippocampus and to attenuate the vascular and cardiac responses mediated by $\alpha$ - and $\beta$-adrenoceptor activation, suggesting that LSD possesses adrenergic activity via $\alpha$ - and $\beta$-adrenoceptor interactions (Palmer and Burks, 1971; Hungen et al., 1975; Tang and Seeman, 1980). Several earlier preclinical studies reported no effects of LSD administration over brain NE levels or turnover (Katz and Kopin, 1969; Leonard and Tonge, 1969; Peters, 1974; Stolk et al., 1974; McCall and Aghajanian, 1980), whereas others reported a slight $(\sim 20 \%)$ decrease in nonstressed and stressed animals (Barchas and Freedman, 1963; Diaz et al., 1968; McGrath and Olverman, 1978). In humans, LSD was reported not to alter the urinary excretion of NE (Hollister and Moore, 1967). Acutely, LSD was shown to facilitate neuronal excitation in the rat facial motor nucleus, potentiating the response to microiontophoretically applied $\mathrm{NE}$ (McCall and Aghajanian, 1980). Moreover, NE-depleted rats were reported to have an attenuated behavioral phenotype in response to LSD, suggesting that NE projections from the LC may indeed be relevant for the behavioral effects of LSD (Geyer et al., 1985). One study investigating the effects of repeated LSD administration (14 days) reported a decrease of brainstem NE levels of $20 \%$ compared with controls (Peters, 1974). LSD was reported not to inhibit NE reuptake (Dengler et al., 1961).

4. Psilocybin. Psilocybin is an $\alpha 2$-adrenoceptor agonist (Marona-Lewicka and Nichols, 1995). Psilocybin decreases available NE levels by up to $25 \%$ in the rat brain in the 4 hours after administration (Stolk et al., 1974). Interestingly, $\mathrm{NE}$ synthesis in the brain was transiently increased after psilocybin administration, whereas normetanephrine, a product of NE turnover, was increased by over 2-fold 1 hour after psilocybin administration, suggesting that aside from affecting the levels of extrasynaptic NE, psilocybin might affect NE turnover (Stolk et al., 1974). Earlier in vitro studies reported that psilocybin increases $\mathrm{NE}$ reuptake (Herblin and O'Brien, 1968), whereas recent studies suggest that psilocybin binds NET, likely inhibiting NE reuptake (Rickli et al., 2016).

5. N,N-Dimethyltryptamine, 5-Methoxy-N,N-dimethyltryptamine, Ayahuasca. Similar to LSD, mescaline, and psilocin, DMT was reported to potentiate the effects of 5-HT and NE in the facial nucleus in rats (McCall and Aghajanian, 1980). One early report found that DMT slightly increases $\mathrm{NE}$ synthesis in the rat forebrain (Waldmeier and Maitre, 1977), whereas another found no effect (Smith, 1977). Neither report found evidence of effects on NE turnover (Smith, 1977; Waldmeier and Maitre, 1977). Ayahuasca administration increases NE levels in the amygdala but not the hippocampus of rats (de Castro-Neto et al., 2013). Moreover, the level of 4hydroxy-3-methoxy mandelic acid, a product of $\mathrm{NE}$ metabolism, was decreased in the amygdala at all doses tested, and in the hippocampus, it was decreased only at the highest doses tested (de Castro-Neto et al., 2013). On the contrary, another study found no changes in the levels of the NE metabolite 3-methoxy-4-hydrohyphenylglycol after repeated ayahuasca administration (Colaço et al., 2020). In humans, ayahuasca administration was reported to increase the urinary excretion of normetanephrine (Riba et al., 2003). Given that harmaline binds adrenoceptors, it is possible that the effects observed in response to ayahuasca administration over NEergic neurotransmission might arise from such interaction (Miralles et al., 2005). Depletion of NE was shown to block and reverse the analgesic effects of 5MeO-DMT, suggesting that 5-MeO-DMT might elicit analgesic effects via modulating the NEergic system (Archer et al., 1985; Alhaider et al., 1993). Lastly, 5MeO-DMT has been suggested to suppress NE reuptake and to increase its turnover (Fuxe et al., 1972; Nagai et al., 2007).

\section{G. Oxytocin}

Although beyond the scope of this work, OT is a neurotransmitter hormone involved in sociability and anxiety that is dysregulated across psychiatric disorders (Panksepp, 1992; Costa et al., 2009; Cochran et al., 2013). Oxytocin is thought to mediate at least partially the empathogenic, antidepressant, and anxiolytic effects elicited by psychedelic compounds and SSRIs (Uvnäs-Moberg et al., 1999; Schindler et al., 2018) via modulating corticolimbic circuits (Lahoud and Maroun, 2013; Sobota et al., 2015; Triana-Del Rio et al., 2019). Transient OT increase and prosocial effects were reported in response to LSD (Schmid et al., 2015a; Duerler et al., 2020) and MDMA (Dumont et al., 2009; Kirkpatrick et al., 2014a,b) in healthy subjects through $5-\mathrm{HT}_{1 \mathrm{~A} / 2 \mathrm{~A}}$ receptor stimulation (Thompson et al., 2007). Such enhancing effects over the oxytocinergic system also observed with MDMA and DOI in preclinical models (Van de Kar et al., 2001; Thompson et al., 2007) suggest that these compounds might be useful for the treatment of ASD (Dolder et al., 2016; Parker et al., 2017). In ASD, lower baseline OT levels 
predict greater sociability improvements after OT administration (Parker et al., 2017), and OT receptor gene variants predict the subjective effects of MDMA (Bershad et al., 2016). Therefore, given that MDMA, LSD, and DOI all increase OT levels, patients with ASD (especially those with low baseline OT levels), might benefit from the OT-enhancing and empathogenic outcomes of these compounds (Van de Kar et al., 2001; Schmid et al., 2015a). Indeed, clinical studies are investigating the utility of MDMA, ketamine, and LSD on ASD (see Supplemental Table 1; Tables 1-4). Moreover, given that OT augmentation in psychotherapeutic settings is beneficial for depression (MacDonald et al., 2013) and PTSD (Koch et al., 2014), future studies should investigate the utility of psychedelic-augmented psychotherapy for these conditions, as it is already the case of MDMA for treatment-resistant PTSD (Mithoefer et al., 2018). Interestingly, it was reported that MDMA leads to an OT-dependent reopening of long-term depression in the NAc, and this represents a critical mechanism for social reward learning (Nardou et al., 2019). It is thus possible that the creation of a temporary neuroplastic window in which trauma can be reprogrammed, and neural circuits reorganized accordingly, underlies the robust improvements in PTSD symptoms observed after MDMA-augmented psychotherapy (Mithoefer et al., 2018, 2019). As hypothesized for other psychedelics, this might be mediated by chromatin rearrangements, leading to changes in gene expression and ultimately behavior (Nardou et al., 2019; Inserra, 2018). Lastly, given that OT is useful in the treatment of migraine headaches (Phillips et al., 2006), the oxytocinergic properties of psychedelic compounds could be at the base of preliminary evidence suggesting that psychedelics might be useful for headache migraines and cluster headaches (Andersson et al., 2017).

\section{Biased Signaling, Biased Phosphoproteomics, and Psychedelic Compounds}

\section{A. Role of Biased Signaling in Pharmacology and Psychedelic-Induced Biased Signaling}

Several lines of evidence suggest that ligands can stabilize different receptor active states, leading to interaction with different cellular signaling proteins to form a range of different complexes, thus leading to the selective activation of some signaling pathways over others. This phenomenon is called "biased signaling" or "functional selectivity" (Luttrell et al., 1999; Urban et al., 2007; Reiter et al., 2012; Kenakin, 2019). GPCRs (such as serotonergic and glutamatergic metabotropic receptors, which are closely involved in the action of psychedelic compounds) can signal through different intracellular cascades and interact with other GPCRs to form homo- and heteroreceptor complexes. This leads to spatial changes in extracellular and intracellular receptor conformations, which magnify the versatility and specificity of GPCRs via 1) altering the repertoire of potential ligands and their affinity; 2) altering the recruitment of intracellular effector proteins, or $\beta$-arrestins; and ultimately 3 ) modifying the downstream transcriptional effects of ligand interaction (Luttrell et al., 1999; Angers et al., 2002; Lopez-Gimenez et al., 2007; Wootten et al., 2018; Dunn et al., 2019).

From a therapeutic point of view, it is important to identify molecules able to selectively activate a specific therapeutic pathway, avoiding side effects (Kenakin and Christopoulos, 2013; Kenakin, 2015). For example, opioids like morphine are commonly used to treat pain; however, prolonged treatment with these compounds leads to the development of tolerance and related opioid use disorder (Gomes et al., 2020), including respiratory depressant effects. Opioid ligands such as morphine produce their analgesic and respiratory depressant effects through activation of the $\mu$-opioid receptor (MOR), a GPCR receptor that signals through activation of Gi/Go proteins and via arrestin signaling (Williams et al., 2013). In particular, several early studies showed that the opioid respiratory depression was mediated by the $\beta$-arrestin 2 signal (Violin et al., 2014 ), thus promoting the development of MOR ligands that do not recruit $\beta$-arrestin 2 to the receptor, with the assumption that this would avoid side effects that are $\beta$-arrestin-dependent. Recent in vitro studies evaluated novel and promising MOR-biased ligands and assessed their pharmacological profile versus existing opioid compounds. It was found that these molecules (including PZM21 and SR-17018) display improved safety profiles that were not due to a reduction in $\beta$-arrestin-mediated signaling but instead to low intrinsic efficacy (Hill et al., 2018; Gillis et al., 2020). However, despite these encouraging results, the potential clinical use of these compounds remains uncertain. Indeed, in vitro research showed that oliceridine, a promising MOR-biased agonist, elicits robust G protein signaling with potency and efficacy similar to that of morphine but with less $\beta$-arrestin 2 recruitment and receptor internalization (DeWire et al., 2013). Initially, the FDA declined the approval of oliceridine in 2018 because of concerns that the analgesic benefits of the drug did not exceed its risks (including respiratory depression) (Azzam et al., 2019). However, the FDA eventually approved the compound in August 2020, claiming that the safety profile of oliceridine is similar to that of other opioids (U.S. Food and Drug Administration, 2020). On the other hand, TRV027, a biased ligand of the angiotensin II type 1 receptor able to prevent the negative effects of typical angiotensin II blockers (including vasoconstriction and reduced renal perfusion), was tested in patients with acute heart failure in a randomized, double-blind, placebo-controlled doseranging trial. Unfortunately, the TRV027 was revealed not to improve the clinical status at 30-day follow-up compared with placebo, confirming the notion that the 
potential clinical application of biased ligands remains unclear (Pang et al., 2017; Sugihara and Burnett, 2017).

Although some evidence is available for psychedelicspecific receptor conformational changes, receptor internalization bias, heteroreceptor-mediated signal transduction, biased gene expression fingerprints, and psychedelic-specific phosphoproteomics changes, the full spectrum of psychedelic-induced signal transduction and signal plasticity remains to be characterized. A recent crystallography study investigated the effects of LSD binding to $5-\mathrm{HT}_{2 \mathrm{~A}}$ and $5-\mathrm{HT}_{2 \mathrm{~B}}$ receptors (Wacker et al., 2017). The authors described conformational receptor changes upon LSD binding (different from those induced by the nonhallucinogenic compound ergoline), leading to the "trapping" of LSD by $5-\mathrm{HT}_{2 \mathrm{~A}}$ and 5$\mathrm{HT}_{2 \mathrm{~B}}$ receptors in their binding pocket, thus contributing to the slow dissociation rate, long half-life, and likely the long-lasting psychoactive effects of this compound (Wacker et al., 2017). The resulting conformational changes of the $5-\mathrm{HT}_{2 \mathrm{~A}}$ receptor lead to strong functional selectivity for $\beta$-arrestin signaling over $\mathrm{Gq}$ signaling (Wacker et al., 2013, 2017). An in vitro study conducted in mouse embryonic fibroblast derived from wild-type and $\beta$-arrestin knockout mice demonstrated that serotonin produces $5-\mathrm{HT}_{2 \mathrm{~A}}$ receptor internalization via $\beta$-arrestin coupled to protein kinases 1 and $2(\operatorname{Erk} 1 / 2)$ phosphorylation activation. On the contrary, when the selective $5-\mathrm{HT}_{2 \mathrm{~A}}$ receptor agonist DOI was tested, the authors found that $\beta$-arrestin is not essential for DOIinduced $5-\mathrm{HT}_{2 \mathrm{~A}}$ receptor desensitization and Erk1/2 activation (Schmid et al., 2008; Perez-Aguilar et al., 2014). Similarly, LSD was shown to have signaling bias at $5-\mathrm{HT}_{2 \mathrm{C}}$ receptors in vitro compared with canonical 5HT signaling (Backstrom et al., 1999). In this study, the authors reported that although 5-HT, LSD, and DOI all promote phosphoinositide hydrolysis and protein kinase $\mathrm{C}$ membrane translocation upon binding, LSD does not promote a detectable calcium release (Backstrom et al., 1999). Moreover, LSD binding to the alternative (fully) edited $5-\mathrm{HT}_{2 \mathrm{C}}$ receptor isoform results in a decreased ability to activate phosphoinositide hydrolysis signaling compared with 5-HT (Backstrom et al., 1999). Intriguingly, a recent article reported the development of a stable HEK 293 T-cell-based bioassay to monitor $\beta$-arrestin 2 recruitment to the $5-\mathrm{HT}_{2 \mathrm{~A}}$ receptor (Pottie et al., 2020). This in vitro bioassay system was used for the functional characterization of 30 phenylalkylamine psychedelics, including 12 phenethylamines (2C-X), 7 phenylisopropylamines (psychedelic amphetamines), and $11 \mathrm{~N}$-benzyl derivatives, thus allowing the comprehensive assessment of the structure-activity relationship within and between the different subclasses. For details, see Pottie et al. (2020).

\section{B. Psychedelics and Homo- and Heteroreceptor Complexes}

Aside from biased signal specificity at individual receptors, signal specificity is also enhanced by the existence of homo- and heteroreceptor complexes that are formed when multiple GPCRs are in close proximity on the plasma membrane and physically interact through their transmembrane domains (Angers et al., 2002; González-Maeso et al., 2008). Similar to what happens with biased signaling, conformational changes of the individual GPCRs that form the complex alter the repertoire of potential ligands, as well as the affinity for intracellular signal transduction proteins, ultimately eliciting specific patterns of gene expression (BorrotoEscuela et al., 2017a).

A crucial receptor-receptor interaction has been described between $5-\mathrm{HT}_{2 \mathrm{~A}}$ and mGluR2 receptors that 1) converges signals from glutamatergic and serotonergic neurotransmission, 2) is increased in schizophrenia and decreased by antipsychotics, and 3) is necessary to induce psychedelic effects in preclinical models (Moreno et al., 2011; Gewirtz and Marek, 2000; Moreno et al., 2016). Indeed, chronic treatment with mGlu2/3 inhibitors decreases the $5-\mathrm{HT}_{2 \mathrm{~A}}$ receptor-dependent hallucinogenic effects of LSD (Moreno et al., 2013). The mechanism for the functional interaction between $5-\mathrm{HT}_{2 \mathrm{~A}}$ and mGluR2 receptor signaling is not completely understood. Despite the presence in the literature of important evidence demonstrating the heterodimerization between these two receptors (González-Maeso et al., 2008; Moreno et al., 2011), this conclusion remains controversial (Delille et al., 2013). Indeed, Delille et al. reviewed the evidence for a heterodimer $5 \mathrm{HT}_{2 \mathrm{~A}}-\mathrm{mGluR2}$ complex showed by González-Maeso et al. (2008). Delille et al. (2013) note that the formation of a heterodimer complex in a recombinant system is not necessarily an evidence and justification for its occurrence in vivo. Moreover, the authors pointed out that several lines of evidence suggest that mGlu2 receptors are expressed at a presynaptic level, whereas the $5-\mathrm{HT}_{2 \mathrm{~A}}$ receptor is mostly expressed postsynaptically. Furthermore, an allosteric interaction of $5-\mathrm{HT}_{2 \mathrm{~A}}$ and mGlu2 receptors has not been independently replicated in any other laboratory. They conclude that until ultrastructural studies can be finalized, the physiological importance of a $5-\mathrm{HT}_{2 \mathrm{~A}}-\mathrm{mGlu} 2$ heterodimer remains questionable and controversial (Nichols, 2016; Murat et al., 2019).

The interaction of psychedelic compounds with these heteroreceptor complexes regulates cortical sensory gating and triggers psychedelic-specific patterns of gene expression, which differ from those activated by nonpsychedelic $5-\mathrm{HT}_{2 \mathrm{~A}}$ receptor agonists (González-Maeso et al., 2003). Gene expression changes triggered by to the nonhallucinogenic $5-\mathrm{HT}_{2 \mathrm{~A}}$ receptor agonist lisuride differ profoundly from those elicited by the hallucinogenic LSD and DOI. For instance, LSD and DOI, but not lisuride, upregulate the expression of Egr-1 and -2 in the somatosensory cortex (Davis et al., 1991; GonzálezMaeso et al., 2003, 2007). A deeper layer of psychedelicinduced signal specificity arises from the fact that 
different psychedelic compounds induce compoundunique transcriptional responses. For example, LSD and psilocybin, but not DOI, upregulate solute carrier family 2, facilitated glucose transporter member 4 (Glut4) and GPCR26 (González-Maeso et al., 2003, 2007) [for an overview of these results, see Supplemental Table 3 in González-Maeso et al. (2007)]. Another heteroreceptor complex of interest, identified in the dorsal and ventral striatum and involved in psychedelicinduced signal transduction, is the $5-\mathrm{HT}_{2 \mathrm{~A}}-\mathrm{D} 2 \mathrm{~L}$ heteroreceptor complex, in which $\mathrm{D} 2 \mathrm{~L}$ indicates the long D2 receptor splice variant (Albizu et al., 2011; BorrotoEscuela et al., 2014).

This complex is of particular interest given that psychedelics mimic some of the positive symptoms of schizophrenia and antipsychotic drugs modulate D2 receptor function (Davis et al., 1991; Carlsson and Carlsson, 2008; Grace, 2016; Jauhar et al., 2019). When psychedelics bind the $5-\mathrm{HT}_{2 \mathrm{~A}}$ receptor of a $5-\mathrm{HT}_{2 \mathrm{~A}}-\mathrm{D} 2 \mathrm{~L}$ complex, an allosteric facilitatory interaction takes place between $5-\mathrm{HT}_{2 \mathrm{~A}}$ and $\mathrm{D} 2 \mathrm{~L}$ receptors that favors D2 receptor signaling to canonical Gi/o-mediated 5$\mathrm{HT}_{2 \mathrm{~A}}$ receptor signaling (Borroto-Escuela et al., 2014). This effect is triggered by the $5-\mathrm{HT}_{2 \mathrm{~A}}$ receptor binding of some psychedelic (LSD and DOI) compounds but not by others (such as 4-Bromo-3,6-dimethoxybenzocyclobuten1-yl)methylamine hydrobromide, also called TCB-2) (Borroto-Escuela et al., 2014). Although the existence of a $5-\mathrm{HT}_{2 \mathrm{~A}}-\mathrm{D} 3$ heteroreceptor complex has not been reported, it is plausible that these two receptors interact given that a D3 receptor antagonist is sufficient to block the stereotypical psychedelic-induced head-twitch response in mice (Perreault et al., 2014). Another possibility is that a $5-\mathrm{HT}_{2 \mathrm{~A}}-\mathrm{D} 2-\mathrm{D} 3$ heteroreceptor complex exists given that both $5-\mathrm{HT}_{2 \mathrm{~A}}-\mathrm{D} 2$ and $\mathrm{D} 2-\mathrm{D} 3$ complexes have been described (Lukasiewicz et al., 2010; Perreault et al., 2014). Dopamine receptors also form other dopamine-dopamine receptor complexes, such as D1D2 and D2-D5, and heterodimers with other receptors, such as D2-NMDA, D2-TAAR1, and D2-cannabinoid 1 receptor [reviewed in Guevara-Lora et al. (2016)]. Importantly, 5- $\mathrm{HT}_{1 \mathrm{~A}}-\mathrm{D} 2$ heteroreceptor complexes have also been reported, and antipsychotics were shown to favor this signaling pathway (Łukasiewicz et al., 2016). Further studies should investigate whether psychedelics interact with, and signal through, these or other homo- and heteroreceptor complexes.

The existence of $5-\mathrm{HT}_{1 \mathrm{~A}}-5-\mathrm{HT}_{2 \mathrm{~A}}$ isoreceptor complexes has been described in the pyramidal cell layer of the dorsal hippocampus and in the ACC (BorrotoEscuela et al., 2017b). The spatial arrangement of these complexes is stress-responsive given that stress decreases their colocalization, potentially via enhancing internalization (Borroto-Escuela et al., 2017b). In these complexes, the binding of specific $5-\mathrm{HT}_{1 \mathrm{~A}}$ receptor agonists was decreased by the steric hindrance of agonist-bound $5-\mathrm{HT}_{2 \mathrm{~A}}$ receptor, whereas this effect was nullified by the $5-\mathrm{HT}_{2 \mathrm{~A}}$ receptor antagonist ketanserin (Borroto-Escuela et al., 2017b). Interestingly, the $5-\mathrm{HT}_{1 \mathrm{~A}}$ receptor is mainly inhibitory and signals through $\mathrm{Gi} / \mathrm{o}$, whereas the $5-\mathrm{HT}_{2 \mathrm{~A}}$ receptor is excitatory and signals through $\mathrm{Gq} / 11$; the intracellular consequences of ligand binding to $5-\mathrm{HT}_{1 \mathrm{~A}}-5-\mathrm{HT}_{2 \mathrm{~A}}$ isoreceptor complexes remain to be fully characterized (Hamon and Blier, 2013). Given that $5-\mathrm{HT}_{1 \mathrm{~A}}$ and $5-\mathrm{HT}_{2 \mathrm{~A}}$ receptor unbalances increase the likelihood of developing psychiatric disorders and given that antidepressant responses rely strongly on the molecular dynamic interactions of these receptors, the $5-\mathrm{HT}_{1 \mathrm{~A}}-5-\mathrm{HT}_{2 \mathrm{~A}}$ isoreceptor complex is of enormous interest in psychiatry. This complex represents a potential driving mechanism in psychiatry disorders and a candidate therapeutic target for drug discovery (Bonson et al., 1996; Celada et al., 2004; Aghajanian, 2009; Yohn et al., 2017).

Other 5-HT isoreceptor complexes have been characterized, such as the heterodimers $5-\mathrm{HT}_{2 \mathrm{~A}}-5-\mathrm{HT}_{2 \mathrm{C}}$ and 5$\mathrm{HT}_{1 \mathrm{~A}}-5-\mathrm{HT}_{7}$ and the homodimers $5-\mathrm{HT}_{1 \mathrm{~A}}-5-\mathrm{HT}_{1 \mathrm{~A}}$ and $5-$ $\mathrm{HT}_{7}-5-\mathrm{HT}_{7}$, although their involvement in psychedelic signaling remains to be elucidated (Renner et al., 2012; Felsing et al., 2018). Given that LSD displays affinity for both $5-\mathrm{HT}_{1 \mathrm{~A}}$ and $5-\mathrm{HT}_{7}$ receptors, it is possible that LSD might have affinity for the $5-\mathrm{HT}_{1 \mathrm{~A}}-5-\mathrm{HT}_{7}$ complex (Crider et al., 2003; Renner et al., 2012). Lastly, glutamate heteroreceptor complexes mGluR1-mGluR5 and mGluR2-mGluR4 exist in glutamatergic synapses (Pandya et al., 2016). Whether psychedelics bind to these complexes remains to be determined. Together, a deeper understanding of psychedelic-induced signal specificity, ligand sites that determine affinity, dissociation rates, and functional potency could be exploited to enhance the therapeutic efficacy (and potentially decrease psychoactivity) of psychedelic compounds in psychiatry, similar to the efforts in identifying more efficacious antipsychotic derivatives (Shah et al., 2019).

\section{Psychedelic-Induced Biased Phosphoproteomics}

Phosphoproteomic refers to the post-translational phosphorylation of proteins, a process that affects their function and is orchestrated by kinases and phosphatases, which modulate a wide range of cellular processes (Riley and Coon, 2016). Aside from psychedelic-biased effects on signal transduction and gene expression, psychedelic-specific phosphoproteomic changes have been reported. For example, DOI modulates the phosphoproteome in a specific fashion compared with the nonhallucinogenic $5-\mathrm{HT}_{2 \mathrm{~A}}$ receptor agonist lisuride (Karaki et al., 2014). The authors of this study found that 16 sites were differentially phosphorylated by DOI, including a hyperphosphorylated intracellular residue relevant for $5-\mathrm{HT}_{2 \mathrm{~A}}$ receptor desensitization and internalization (Ser ${ }^{280}$ ) (Karaki et al., 2014). Such biased phosphorylation dynamics may underlie the decreased $5-\mathrm{HT}_{2 \mathrm{~A}}$ receptor desensitization and internalization elicited by psychedelic (as opposed to nonpsychedelic) 
$5-\mathrm{HT}_{2 \mathrm{~A}}$ receptor agonists (Raote et al., 2013; Karaki et al., 2014). Although in that study it was not possible to unequivocally determine the molecular events leading to the specific phosphoproteomics changes observed, one potential explanation could be that the psychedelicstabilized $5-\mathrm{HT}_{2 \mathrm{~A}}$ receptor conformation might modulate protein kinase $\mathrm{C}$ access, leading to a psychedelicspecific phosphorylation fingerprint (Karaki et al., 2014). Interestingly, it was also reported that mGluR2 undergoes specific ligand-induced phosphorylation at $\mathrm{Ser}^{843}$ only in the presence of $5-\mathrm{HT}_{2 \mathrm{~A}}$ receptor, which could represent a key molecular interaction mediating the psychedelic crosstalk between these two receptors and might be involved in psychedelic-initiated therapeutic improvements (Murat et al., 2019). Whether psychedelics affect $5-\mathrm{HT}_{2 \mathrm{~A}}$ receptor-mediated mGluR2 phosphorylation remains to be ascertained. Further in vitro and in vivo studies should assess psychedelicinduced phosphoproteomics changes in a compound-, regimen-, and brain region-specific fashion while assessing whether these changes can be detected peripherally as peripheral biomarkers of psychedelicinduced brain phosphoproteomics changes.

Although our understanding of psychedelic-specific intracellular cascades is increasing, it seems plausible that the full pharmacological profile of psychedelic compounds is far more complex than previously thought, requiring the activation of 1) multiple homoand heteroreceptors complexes, 2) biased signaling cascades, and 3) biased post-translational protein modifications leading to 4) specific gene transcription programs, ultimately eliciting beneficial effects on mood and behavior (Celada et al., 2004; Reissig et al., 2005; Savitz et al., 2009; Wesnes et al., 2016). Independent from whether psychedelics will be approved as medicines, the knowledge generated is creating a unique opportunity for the development of novel, more efficacious designer drugs with structural analogy to psychedelic compounds (McLean et al., 2006; Zanos et al., 2017; Kenakin, 2019). Such a paradigm is already being explored for the design of a ketamine- and opioid-like analgesic with reduced side effects and could be employed in designing antidepressant and anxiolytic molecules with enhanced efficacy and reduced side effects (Manglik et al., 2016; Conibear and Kelly, 2019; Harvey et al., 2019). If skillfully harnessed, the knowledge generated could drastically improve our pharmacotherapeutic toolbox in psychiatry.

\section{Going Beyond Receptors: Neuronal Circuits Activated by Psychedelic Drugs}

Until now, research on the effects of psychedelics has mostly focused on the single effects of a compound on specific neurotransmitters and neuroplastic or neuroinflammatory mediators. However, the complex psychological effects triggered by psychedelics, such as increased empathy, overcoming of trauma in PTSD, and increased capacity of insight in psychotherapy, suggest that psychedelics can activate specific neuronal circuits in the brain. Recently, more emphasis has been placed on the importance of assessing the effects of psychedelics within a more comprehensive framework. This can be achieved, for example, by employing multiple imaging techniques to assess neural activity and directed and undirected functional connectivity while also appreciating the effects on neurotransmitter concentration levels (Barnett et al., 2020; Kringelbach et al., 2020). This type of approach could help illustrate a range of fast-onset psychological effects of psychedelics that are likely not due to long-term neuroplasticity or epigenetics.

Several neural circuits have been suggested that might drive the therapeutic improvements elicited by psychedelics. Although some evidence is available, more research is needed to understand how each circuit is connected to specific effects evoked by psychedelics. Rather than mutually exclusive, it is likely that a combination of the circuits so far proposed coupled with purely immunomodulatory and neurotrophic actions and subjective meaningful experiences concur to elicit the observed clinical improvements. The activation of 5$\mathrm{HT}_{2 \mathrm{~A}}$ receptors and related GPCRs appear to play a major role in the behavioral effects of serotonergic hallucinogens. The effects of $5-\mathrm{HT}_{2 \mathrm{~A}}$ receptor activation on layer 5 and 6 pyramidal neurons and the resulting electrical and chemical excitatory output may be responsible for mediating a range of therapeutic improvements linked to limbic circuitry, which involves connectivity between the PFC, striatum, thalamus, claustrum, amygdala, and hippocampus (Willins et al., 1997; Riba et al., 2006; Béïque et al., 2007; Anand et al., 2009; Surget et al., 2009; Carhart-Harris et al., 2016c; Peters et al., 2016; Lewis et al., 2017; Marek, 2018; Posner et al., 2014).

\section{A. Effects on the Default Mode Network Hub Functioning, and the "Reset" Model}

The DMN refers to a circuit involving several brain regions, such as the ventromedial and dorsal $\mathrm{PFC}$, the PCC, and discrete regions of the hippocampal formation (Buckner et al., 2008). The DMN is mostly active in resting state and when attention is not directed toward an external stimulus. DMN activity has been associated with introspection, autobiographical memory retrieval, internal mentation, the separation of one's self from others and the surrounding world, and "time travel" (such as remembering the past and envisioning the future) (Andrews-Hanna, 2012; Buckner et al., 2008; Østby et al., 2012; Li et al., 2014). The DMN is also thought to be involved in the construction of "ego," or "self" (Lebedev et al., 2015). The DMN is overactive in several psychiatric disorders, including anxiety (Coutinho et al., 2016), depression (Hamilton et al., 
2011), and obsessive-compulsive disorder (Beucke et al., 2014). Such overactivity is understood to shape feelings of rumination, worthlessness, and negative selfjudgement (Buckner et al., 2008; Sheline et al., 2009).

Psychedelics such as psilocybin (Carhart-Harris et al., 2017), ayahuasca (Palhano-Fontes et al., 2015), and LSD (Muthukumaraswamy et al., 2013; CarhartHarris et al., 2016c; Tagliazucchi et al., 2016; Müller et al., 2018) decrease DMN activity. For example, LSD and psilocybin decrease connectivity between the PCC and the parahippocampus, and this correlates with selfreported scores of "ego dissolution" and visual imagery (Lebedev et al., 2015; Carhart-Harris et al., 2016c; Kaelen et al., 2016). Similarly, disrupting PCC connectivity via means of electrical stimulation in men induces dream-like hallucinations and detachment from the surrounding world, effects which resemble the psychedelic state (Herbet et al., 2014). It has been suggested that a "reset" mechanism might take place in which the acute disintegration of canonical information processing within DMN substrates allows for a subsequent resumption and reintegration of normal brain function (Carhart-Harris et al., 2017). One study has investigated the long-lasting effects of psilocybin-assisted mindfulness training, observing that higher scores of self-reported "oceanic boundlessness" during psilocybin acute effects were positively correlated with postretreat decoupling of functional connectivity between the mPFC and ACC during meditation. This finding stresses the role of synaptic plasticity as a potential mechanism of long-term psychological improvements after psychedelic therapy (Smigielski et al., 2019).

\section{B. The Cortico-Striato-Thalamo-Cortical Model}

The activation of presynaptic $5-\mathrm{HT}_{2 \mathrm{~A}}$ receptors in deep-layer cortical neurons is thought to mediate the psychedelic effects of LSD and other serotonergic psychedelics (Titeler et al., 1988; Vandermaelen and Aghajanian, 1983; Jakab and Goldman-Rakic, 1998; Peyron et al., 1998; Vollenweider and Kometer, 2010; Duerler et al., 2020; Puig et al., 2003). It has been suggested that this interaction might be the driver of the cortico-striato-thalamo-cortical (CSTC) feedback loop modulation observed in imaging studies (Höflich et al., 2015; Müller et al., 2017; Preller et al., 2018, 2019). Cortical layer 6 contains corticothalamic glutamatergic neurons, which excite thalamocortical relay neurons while disynaptically inhibiting them via exciting GABAergic neurons in the RT (Jones, 2001; Zhang and Jones, 2004; Riga et al., 2018). GABAergic neurons in the $\mathrm{RT}$ represent a main inhibitory input to the thalamus and, by means of depolarizing $\mathrm{GABA}_{\mathrm{A}}$ receptor potential-induced bursts, are involved in the generation of brain electrical oscillations and sleep spindles (Bazhenov et al., 1999; Steriade, 2003). RT dysfunction is involved in several psychiatric disorders, including SCZ, ASD, and BD (Wells et al., 2016;
Ferrarelli and Tononi, 2017; Steullet et al., 2018). Neurons in the RT provide finely tuned spatiotemporal control of thalamocortical relay cells, thereby gating thalamocortical information flow (Jones, 2001; Wang et al., 2010). This pathway, which has been hypothesized to generate consciousness (Alkire et al., 2008; Min, 2010; Ward, 2011; Herrera et al., 2016), might represent one of the main neurobiological substrates generating the wide range of consciousness-altering effects of psychedelic compounds.

In other words, psychedelic compounds might "open the gate" of consciousness (Scruggs et al., 2000; Marek et al., 2001; Geyer and Vollenweider, 2008; Müller et al., 2017; Preller et al., 2019) via allowing the thalamocortical transfer of information that might otherwise be blocked by circuits of selective attention, including the RT (McAlonan et al., 2000, 2006). A potential mechanism that might mediate such effects is the presence of serotonergic projections from the DRN (Rodriguez et al., 2011) and norepinephrinergic projections from the locus coeruleus (Asanuma, 1992), which by releasing monoamines, keep RT neurons in a depolarized state, facilitating the generation of T-type calcium channelmediated bursting (Bosch-Bouju et al., 2013). Given that LSD decreases serotonergic firing in the DRN (Aghajanian and Vandermaelen, 1982; De Gregorio et al., 2016b), it is possible that the LSD-induced decrease of serotonergic input from the DRN leads to a hyperpolarization of RT neurons that express 5-HTRs (Goitia et al., 2016), decreasing bursting activity and ultimately decreasing the inhibitory influence of the RT on thalamocortical relay cells and thereby "opening the gate." The resulting increased thalamocortical connectivity $\left(5-\mathrm{HT}_{2 \mathrm{~A}}\right.$ receptor-independent) is associated with decreased cortico-striato-thalamo connectivity $\left(5-\mathrm{HT}_{2 \mathrm{~A}}\right.$ receptor-dependent) (Geyer and Vollenweider, 2008; Müller et al., 2017; Preller et al., 2019). The latter mechanism might be responsible for the decreased global thalamic activity after the administration of psychedelics (Carhart-Harris et al., 2012; Lewis et al., 2017; Preller et al., 2019), whereas the former could be responsible for the increased thalamic functional connectivity observed in human imaging studies (Tagliazucchi et al., 2016; Müller et al., 2017; Vlisides et al., 2017; Preller et al., 2019; Barnett et al., 2020).

SSRIs similarly increase thalamic functional connectivity, pointing out another common mechanism in the action of SSRIs and psychedelics (Schaefer et al., 2014). Given that psychedelics enhance thalamocortical functional connectivity, and given that thalamocortical activation is required for memory retrieval (Staudigl et al., 2012; Müller et al., 2017; Preller et al., 2018, 2019) and the formation of new memories (Sweeney-Reed et al., 2014), it cannot be excluded that modulation of this circuit by psychedelics might be involved in the therapeutic improvements observed in populations with PTSD who report an enhancement of fear 
extinction and memory reconsolidation after the administration of psychedelics in clinical settings.

\section{The Claustrum}

Other areas that deserve more systematic investigations include the claustrum (Smythies et al., 2012) and the thalamoclaustral (Vertes et al., 2006; Vertes and Hoover, 2008) and cortico-claustro-cortical circuits (Olson and Graybiel, 1980). The claustrum is an area rich in $5-\mathrm{HT}_{2 \mathrm{~A}}$ receptors (Watts et al., 1994), which are internalized upon psychedelic binding (Martin and Nichols, 2016). The claustrum has extensive inhibitory functional connectivity with cortical areas related to the top-down control of cognitive function and emotions, such as the prelimbic prefrontal cortex and the anterior cingulate cortex (Jackson et al., 2018; White et al., 2018; Krimmel et al., 2019). Indeed, DOI administration induces cFOS expression in a subpopulation of claustral neurons that express $5-\mathrm{HT}_{2 \mathrm{~A}}$ receptors (Martin and Nichols, 2016). Network theoretical analyses revealed that the claustrum is a primary contributor to global brain network architecture and that significant connectivity exists between the claustrum, the frontal lobe, and cingulate regions (Torgerson et al., 2015; Wang et al., 2017b; Chia et al., 2020). Therefore, the claustrum could be one of the areas where psychedelics activate neural information for altered states of consciousness and awareness, possibly via integrating and rendering coherent multiple frequencies of neural oscillations (Crick and Koch, 2005; Goll et al., 2015).

Indeed, a recent study reported that acute psilocybin significantly decreases claustral activity and connectivity with brain areas involved in emotions, memory, and attention (Barrett et al., 2020b). Specifically, psilocybin decreased the amplitude of low-frequency fluctuations and the variance of blood oxygenation level-dependent signal in the left and right claustrum. Moreover, acute psilocybin administration decreased functional connectivity of the right claustrum with the DMN, increased right-claustrum connectivity with the frontoparietal task control network (FPTC), and decreased leftclaustrum connectivity with the FPTC (Barrett et al., 2020b). Interestingly, DMN integrity was associated with right-claustrum connectivity with the DMN, whereas FPTC integrity and modularity were associated with right-claustrum and left-claustrum connectivity with the FPTC (Barrett et al., 2020b).

The organization of the claustrum resembles that of the thalamus (Olson and Graybiel, 1980), suggesting that it might serve similar functions in terms of information processing and gating. Similarly to the thalamus, which is able to suppress distractors to attend to salient stimuli, the claustrum was shown to become activated in situations of cognitive conflict, which require changes in cognitive strategy, and may therefore be a brain area involved in cognitive plasticity and adaptability (Krimmel et al., 2019). Moreover, similarly to the thalamocortical circuit, which can inhibit the cortex, creating a state of unconsciousness, the claustrum might be involved in sleep via feedforward inhibition of the cortex (Jackson et al., 2018; Norimoto et al., 2020). Given that the final output between excitation and inhibition of the cortex is involved in a range of behaviors, such as reward, anxiety, and sociability (Jackson et al., 2018), and given that psychedelics affect claustral activity and connectivity (Barrett et al., 2020b), the modulation of claustral activity and connectivity might represent a valuable novel therapeutic strategy for the treatment of addiction, psychiatric disorders, and disorders characterized by sociability impairments, such as ASD.

Together, the modulatory effects of psychedelics on the DMN, CSTC, and claustral circuits seem to be closely related to experiencing 1) "mystical-type experiences" (Griffiths et al., 2011, 2018; Barrett and Griffiths, 2018), often considered among the 10 most meaningful experiences of one's life (Schmid and Liechti, 2018); 2) "emotional breakthrough" episodes (Roseman et al., 2019), which have been suggested to act as a "reset" switch; 3) psychedelic-induced enhancement of psychological flexibility (Davis et al., 2020); 4) creative thinking (Barrett et al., 2020b; Girn et al., 2020); and 5) increased acceptance (Girn et al., 2020). These states might mediate the anxiolytic and antidepressant effects of psychedelic compounds (Carhart-Harris et al., 2017; Murphy-Beiner and Soar, 2020).

\section{Effects of Psychedelics on Sleep}

Only a few studies investigated the effects of psychedelics on sleep. For example, daytime ayahuasca administration to healthy volunteers decreased the percentage and duration of rapid eye movement (REM) sleep the night of administration while decreasing the number of periods and increasing the duration of nonrapid eye movement sleep and the number of sleep cycles (Barbanoj et al., 2008). Moreover, individuals receiving ayahuasca showed a trend toward increased REM sleep onset latency and a trend toward increased total sleep time, especially in stage 2 (Barbanoj et al., 2008). Nevertheless, spectral analysis revealed that ayahuasca increased power in the high frequency range (limited to the $15-20 \mathrm{~Hz}$ band) mainly in stage 2 while enhancing slow-wave sleep (Barbanoj et al., 2008). These effects were not accompanied by changes in subjective measures of sleep quality (Barbanoj et al., 2008).

Similarly, LSD administered just before bedtime was shown to increase the duration of either the first or second REM periods (results consistent for each patient on different nights) up to $245 \%$ compared with controls in some patients, with a rebound effect in the rest of the REM periods of the night, which were fewer and briefer (Green, 1965; Muzio et al., 1966; Torda, 1968). Much like patients receiving ayahuasca, REM sleep onset was 
delayed by LSD administration (Green, 1965). In another subpopulation of patients, LSD induced arousal episodes that significantly disrupted sleep architecture and sleep quality (Muzio et al., 1966). Interestingly, the eye movements during REM sleep were slower and fewer after LSD (Muzio et al., 1966). In this study, sleep architecture was restored the following night (Muzio et al., 1966). The authors of this study reported the existence of a subjective LSD dose range within which a high probability of prolonging REM sleep exists; however, exceeding this dose results in an arousal-like effect (Muzio et al., 1966). Preclinical studies similarly observed an increase in wakefulness, a dose-dependent increase in REM sleep onset latency, a large decrease in REM sleep, and a smaller decrease in non-rapid eye movement sleep (Depoortere and Loew, 1971; Stern et al., 1972; Kay and Martin, 1978). LSD administration during sleep was reported to increase hippocampal theta activity during REM sleep in response to LSD administration during sleep (Bílková et al., 1971a,b). Aside from acute administration studies, few studies have investigated the long-term effects of psychedelics on sleep. Cats that received LSD once displayed an increase in low-voltage-frequency sleep in the days after LSD administration, suggesting that similar subacute effects might take place in humans, which might be correlated with therapeutic improvement (Hobson, 1964).

Indirect evidence that dissociative anesthetics might affect sleep is available from studies investigating the effects of ketamine and PCP on the RT. Both ketamine and PCP were shown to decrease the activity of RT GABAergic neurons, shifting the firing mode from burst to tonic, thus eliciting an arousal-like effect (TroyanoRodriguez et al., 2014; Mahdavi et al., 2020). Future studies should similarly investigate the effects of serotonergic psychedelics over the RT. Together, given that REM sleep is involved with associative thinking, creativity, memory consolidation, fear extinction, and neuroplasticity (Landmann et al., 2015; Ravassard et al., 2015), and given that psychedelics affect sleep, it cannot be excluded that at least some of the therapeutic benefits of psychedelics might be mediated by their effects over sleep (Froese et al., 2018).

\section{Long-Term Neurobiological and Psychological Effects of Psychedelic Compounds}

Although there is a paucity of studies investigating the long-term outcomes of psychedelic use in the general population, the available evidence suggests no increase in psychopathology and a decreased incidence of psychiatric conditions in subjects that have ingested psychedelics multiple times during their lifetime (Halpern et al., 2005; Bouso et al., 2015; Hendricks et al., 2015; Johansen and Krebs, 2015; Argento et al., 2017; Ly et al., 2018). Potentially, the most remarkable effect of repeated lifetime exposure to psychedelic compounds is a decrease in suicidal ideation and suicidal behavior (Hendricks et al., 2015; Argento et al., 2017; Zeifman et al., 2019, 2020). Aside from the improvement in depression, anxiety, and compulsion domains discussed so far, the enhancement of several behavioral, social, and cognitive domains have been reported after psychedelic exposure. These include reduced psychological distress (Hendricks et al., 2015), decreased opioid dependence (Pisano et al., 2017; Argento et al., 2019; Garcia-Romeu et al., 2020), decreased criminal behavior (Hendricks et al., 2018), enhanced creative divergent thinking and problem-solving skills (Kuypers et al., 2016; Sweat et al., 2016), proenvironmental behavior via increased nature relatedness (Forstmann and Sagioglou, 2017), and a decreased incidence of extreme political views (Nour et al., 2017; Lyons and Carhart-Harris, 2018).

\section{A. N,N-Dimethyltryptamine, 5-Methoxy-N,N- dimethyltryptamine, and Ayahuasca}

The most studied psychedelic compound in terms of long-term effects arising from the repeated ingestion of a serotonergic psychedelic is probably ayahuasca. This stems from the fact that many people in the Amazon Basin, and more recently worldwide, use ayahuasca in religious ceremonies (Labate and Feeney, 2012). This phenomenon allows for the study of a population of individuals who ingest a psychedelic hundreds if not thousands of times in their lifetime and the investigation of long-term neurobiological changes that might arise from chronic use. In terms of acute psychological changes arising from acute ayahuasca administration, rapid improvements in depression scores have been reported in treatment-resistant depression (PalhanoFontes et al., 2019) and recurrent depression (Osório et al., 2015; Sanches et al., 2016). Another study reported a statistical trend toward decreased suicidality in patients from these trials (Zeifman et al., 2019). Four weekly sessions of ayahuasca have been described to increase "acceptance capacities" scores in the nonjudging subscale of the Five Facet Mindfulness Questionnaire (Soler et al., 2018). Recently, it was reported that a 12-day ayahuasca retreat in traditional settings significantly reduces neuroticism scores in an inversely proportional fashion to the intensity and quality of the mystical experiences associated, and these changes are still appreciable at 6-month follow-up (Netzband et al., 2020). Agreeableness was also increased in this cohort, corroborating recent research on the acute prosocial effects elicited by LSD in humans (Duerler et al., 2020; Netzband et al., 2020). Interestingly, adolescents who regularly ingest ayahuasca scored lower for psychiatric symptoms such as anxiety, body dysmorphism, and attentional problems compared with controls, suggesting no psychiatric sequelae arising from regular consumption of ayahuasca during adolescence (Da Silveira et al., 2005). However, how acute or repeated ayahuasca 
intake during childhood and/or adolescence might affect individuals in adulthood-positively or negativelyremains to be determined. Future longitudinal or retrospective studies should aim to answer this research question.

Long-term neurobiological changes such as increased ACC and decreased PCC thickness have been observed in ayahuasca users (Bouso et al., 2015). PCC thickness was positively correlated with greater consumption (Bouso et al., 2015). These changes are consistent with the acute effects of ayahuasca on the DMN (PalhanoFontes et al., 2015), which might induce plasticity in these brain regions. Individuals who regularly consume ayahuasca score lower in psychometric tests for depression and confusion and higher for personality trait agreeableness, openness, and life quality, and these changes are accompanied by recent and lifetime decreased alcohol use and abuse (Barbosa et al., 2016; Garcia-Romeu et al., 2019). No neuropsychological impairment or psychopathology was associated with these changes. Interestingly, lower drug abuse rates and increased spirituality were observed in ayahuasca users (Bouso et al., 2015). Another study found that long-term psychedelic users performed better in neuropsychological tasks, possibly suggesting a state of cognitive enhancement (Bouso et al., 2012). A preclinical study found that ayahuasca consumption during pregnancy and lactation results in decreased anxiety and decreased the interest for social interactions in the offspring, suggesting potential neuromodulatory effects in the fetus (de Oliveira et al., 2011). Inhalation of 5-MeODMT has been reported to occasion mystical experiences associated with almost immediate improvements in depression and anxiety scores with enduring positive effects (Davis et al., 2019) and decreased drug use (Garcia-Romeu et al., 2020; Uthaug et al., 2020). Lastly, a study investigating platelet SERT found an upregulation in long-term ayahuasca drinkers, suggesting that long-term psychedelic use might induce plasticity mechanisms in the serotonergic system, potentially as a result of the long-term potentiation effect of ayahuasca over serotonergic neurotransmission, 5-HT levels, and SERT (Callaway et al., 1994).

\section{B. Lysergic Acid Diethylamide}

A study investigating the long-term effects (1 and 12 months) of a single LSD administration reported improvements in mood, life attitude, and well-being/life satisfaction and no changes in negative attitude, antisocial effects, or negative behaviors resulting from LSD (Schmid and Liechti, 2018). In preclinical studies, a single high dose of LSD does not affect cortical 5$\mathrm{HT}_{1 \mathrm{~A}}$ and 5- $\mathrm{HT}_{2 \mathrm{~A}}$ receptors, whereas repeated administration selectively downregulates the cortical $5-\mathrm{HT}_{2 \mathrm{~A}}$ receptor but not the receptors $5-\mathrm{HT}_{1 \mathrm{~A}}, 5-\mathrm{HT}_{1 \mathrm{~B}}$, $\beta$-adrenergic receptor, $\alpha$-adrenergic receptor 1 and 2, D2 receptor, and SERT (Buckholtz et al., 1990; Gresch et al., 2005). These changes were reversible 4 days after the last administration (Buckholtz et al., 1990). Interestingly, patients with depression have increased 5$\mathrm{HT}_{2 \mathrm{~A}}$ receptor expression in the $\mathrm{mPFC}$ (Meyer et al., 2003; Shelton et al., 2009), and antidepressant compounds desensitize the cortical $5-\mathrm{HT}_{2 \mathrm{~A}}$ receptor (Gómez-Gil et al., 2004; Yamauchi et al., 2006) and decrease its membrane localization (Meyer et al., 2003). Such influences of LSD on 5- $\mathrm{HT}_{2 \mathrm{~A}}$ receptor dynamics, which might be shared by other psychedelic compounds, are likely involved in the anxiolytic and antidepressant mechanism of action of LSD (Gasser et al., 2014, 2015). In preclinical studies, one dose of LSD elicited antidepressantlike effects that were noticeable after 5 weeks in a rodent model of depression (Hibicke et al., 2020). Repeated LSD administration elicited antidepressant-like effects in a preclinical model of antidepressant response without affecting controls, suggesting that although the improvements elicited by LSD might be noticeable in disease states, they might not be so remarkable in homeostasis (Buchborn et al., 2014). Evidence of LSD-induced deleterious neurobiological changes is available from preclinical models of SCZ in which chronic administration of high doses of LSD induces changes in gene expression, such as increased D2 receptor and nuclear orphan receptor and decreased $5-\mathrm{HT}_{2 \mathrm{C}}$ receptor, resembling schizophrenic brains (Marona-Lewicka et al., 2011). No evidence of neuronal damage arising from the administration of clinically relevant doses of LSD is available.

\section{3,4-Methylenedioxymethamphetamine}

MDMA potentially has a higher risk of abuse and long-term adverse neurobiological outcomes when used chronically. Binge abuse of this substance (such as the repeated self-administration of increasing doses due to acute tolerance) can lead to hyperthermia, cardiac arrhythmias, seizures, intracranial hemorrhage, hepatotoxicity, renal failure, and rhabdomyolysis (Hegadoren et al., 1999; Green et al., 2003; Hall and Henry, 2006). Long-term MDMA abuse can have neurotoxic effects on 5-HT and DA neurons and can therefore increase the likelihood of developing psychiatric disorders while decreasing the efficacy of classic psychiatric medications (Montoya et al., 2002; Thomasius et al., 2005; Durkin et al., 2008). However, at clinical doses, the toxicity arising from MDMA administration seems low (Pantoni and Anagnostaras, 2019) (also see the MDMA section of Psychedelic Compounds as Neuroplastic Agents in this review for more details). Importantly, repeated administration of MDMA during adolescence leads to extensive neuroadaptive changes in glutamatergic-related gene expression in corticolimbic structures (KindlundhHogberg et al., 2008). For example, GluR2, mGluR1, mGluR5, NR1, NR2A, NR2B, EAAT1, and EAAT2 were increased in the cortex, whereas GluR3, NR2A, and NR2B receptor subunits were increased in the caudate putamen 
(Kindlundh-Hogberg et al., 2008). GluR1 transcripts levels were reduced in the hippocampus, whereas GluR1, GluR3, mGluR1, and mGluR3 were increased in the hypothalamus (Kindlundh-Hogberg et al., 2008). These findings suggest important neurobiological adaptation within the glutamatergic system, which might mediate the deleterious effects of MDMA when abused at a young age in recreational settings (Kindlundh-Hogberg et al., 2008). Future studies should elucidate whether a similar remodulation of gene expression takes place at clinically relevant doses (Pantoni and Anagnostaras, 2019) and, if so, whether it is possible to detect these changes peripherally in clinical trials participants as an index of potential neurotoxic effects. As suggested above, the possibility of administering a neuroprotective drug concomitantly to MDMA in clinical settings to alleviate potential neurotoxic effects should be investigated (Tourino et al., 2010).

\section{Psilocybin}

Psilocybin has been widely studied in terms of mystical and peak experiences, which seem to mediate and predict at least partially clinical improvements after administration (Griffiths et al., 2008). No main negative psychopathological outcomes seem to arise after the administration of psilocybin in clinical settings (Studerus et al., 2011; Davis et al., 2020). Similarly to LSD, psilocybin elicits antidepressant and statedependent anxiolytic effects in preclinical models, possibly because of the serotonergic and noradrenergic effects, which are detectable 5 weeks after administration (Mahmoudi et al., 2018; Hibicke et al., 2020). Clinical studies corroborate preclinical findings. In fact, psilocybin induces sustained antidepressant and anxiolytic effects, which are detectable up to 6 months after administration (Grob et al., 2011; Carhart-Harris et al., 2016a, 2018a; Griffiths et al., 2016; Ross et al., 2016). Interestingly, 1) the quality of the subjective experience during dosing sessions, 2) increased functional connectivity between the ventromedial prefrontal cortex and bilateral inferior lateral parietal cortex, and 3) decreased functional connectivity between the parahippocampal formation and the PFC predict clinical improvement (Carhart-Harris et al., 2017). Patients with treatmentresistant MDD displayed increased brain-measured emotional responses after psilocybin, suggesting that neuroadaptive processes take place in response to psilocybin treatment, which might help patients reconnect with their emotions (Roseman et al., 2018). Indeed, psilocybin induces brain network and emotional plasticity which are noticeable one week after a single administration but that tend to rebound to baseline 1 month after treatment, while the global increase in functional connectivity is still appreciable after 1 month (Barrett et al., 2020a). Interestingly, psilocybin administration was also correlated with decreased authoritarian views and increased relatedness to nature (Lyons and CarhartHarris, 2018). Concerning alcohol and substance abuse, long-term improvements have been observed after psilocybin, which are indicative of potential usefulness of this approach for the abuse of alcohol, tobacco, and other substances (Garcia-Romeu et al., 2014; Johnson et al., 2014, 2017; Bogenschutz et al., 2015; Nielson et al., 2018). Studies are underway that aim to assess whether psilocybin treatment is feasible, tolerable, and efficacious for the treatment of eating disorders (ClinicalTrials.gov identifier: NCT04052568).

\section{E. Ketamine}

As discussed throughout this review, ketamine elicits rapid, sustained, and long-lasting antidepressant effects in adolescents and adults diagnosed with treatment-refractory MDD (Zarate et al., 2006; Price et al., 2009; aan het Rot et al., 2010; Mathew et al., 2010; Murrough et al., 2013b, 2015; Ionescu et al., 2014; Lapidus et al., 2014; Wan et al., 2015; Cusin et al., 2017; Kiraly et al., 2017; Cullen et al., 2018; Evans et al., 2018a,b; Vidal et al., 2018; Nugent et al., 2019). Importantly, rapid and sustained improvements in suicidality have also been reported (Grunebaum et al., 2018; Sinyor et al., 2018; Domany et al., 2020), suggesting that ketamine might be useful in patients with suicidal ideation and behavior. The antidepressant effects of ketamine are comparable to those of electroconvulsive therapy but have faster onset (Basso et al., 2020). Importantly, greater adverse events can be encountered when ketamine is used instead of other anesthetics for electroconvulsive therapy (Yen et al., 2015; Carspecken et al., 2018). Ketamine also elicits rapid and sustained improvements in PTSD symptoms (Feder et al., 2014; Albott et al., 2018). Given that ketamine may be used in depressed woman who undergo cesarean section to decrease the likelihood of postnatal depression, potential deleterious effects of ketamine on the newborn are being assessed (ClinicalTrials.gov identifier: NCT04285684, NCT03927378, NCT03336541). Ketamine also seems promising for the treatment of alcohol and substance abuse. Decreased cue-induced craving and risk of relapse have been observed after ketamine administration (Dakwar et al., 2014, 2017, 2019, 2020). Suggesting long-term deleterious effects of ketamine abuse on sociability, decreased levels of circulating oxytocin have been described in ketamine-dependent individuals in the early phases of abstinence that were still present 2 weeks after (Huang et al., 2018). Future studies should assess whether this is true when employing clinically relevant regimens. Lastly, given that the antidepressant effects of ketamine require opioid system activation, further studies should investigate potential deleterious effects on the opioidergic system (Williams et al., 2018).

\section{Side Effects of Psychedelic Compounds}

Overall, psychedelics administered in controlled clinical settings appear nonaddictive and present mild, 
transient side effects. There are no reports of deaths after the ingestion of medical-grade psychedelic compounds in controlled settings (Nichols, 2016; Nichols and Grob, 2018). However, there are several concerns that need to be considered in the drug development of psychedelics. First, it seems that psychedelic drugs are strongly influenced by the context and environment (setting), and negative acute or long-term effects such as dysphoric or maladaptive/dysfunctional responses can be experienced by individuals who ingest psychedelics, which might warrant the intervention of a trained therapist (Strassman, 1984; Krebs and Johansen, 2013; Nichols, 2016). The following section aims to discuss potential mild to moderate physical and psychological side effects that can be encountered after the administration of psychedelic compounds in controlled clinical settings and mild, severe, and potentially lifethreatening side effects that can be encountered after the ingestion of illicit psychedelic compounds in uncontrolled settings.

\section{A. Acute Side Effects Encountered in Clinical Trials}

1. Acute Physical Side Effects Encountered in Clinical Trials. Mild to moderate acute physical side effects have been reported by individuals receiving humangrade psychedelic compounds in controlled clinical settings. The most common include 1) headaches; 2) nausea and vomiting (especially when ayahuasca or psilocybin is administered); and increased 5) blood pressure, 6) heart rate, and 7) body temperature (Vollenweider et al., 1999b; Carhart-Harris et al., 2012; Johnson et al., 2012; Murrough et al., 2013b; Feder et al., 2014; Palhano-Fontes et al., 2015, 2019; Schmid et al., 2015a; Griffiths et al., 2016; Nichols, 2016; Mithoefer et al., 2018). The cardiovascular effects elicited by psychedelic compounds are likely mediated by their serotonergic action and should not be overlooked, especially in patients with pre-existing cardiac conditions or a family history of cardiovascular disease.

2. Acute Psychological Side Effects Encountered in Clinical Trials. So far, psychedelics have been considered physiologically and psychologically safe when administered acutely in controlled clinical settings. In healthy patients, the administration of medical-grade psychedelics in controlled clinical settings induces a transient psychosis-like state, which can be accompanied by hallucinations, sense of unity and transcendence, mystical experiences, feelings of bliss and boundlessness, dissociation, derealization, revelations, and the re-experiencing of traumatic memories, which wear off as the compound is metabolized (Nichols, 2016; De Gregorio et al., 2018). Such acute psychological effects might be mediated by the activation of the serotonergic system and HPA axis, effects shared by almost all psychedelics (Vollenweider et al., 1999b; Carhart-Harris et al., 2012; Johnson et al., 2012; Murrough et al., 2013b; Feder et al., 2014; Palhano-
Fontes et al., 2015, 2019; Schmid et al., 2015a; Griffiths et al., 2016; Nichols, 2016; Mithoefer et al., 2018).

Given that the safety concerns largely revolve around the psychological rather than physiological effects of psychedelics, interpersonal support should be available during clinical administration to address psychological distress that might arise. Crucially, since psychedelics can facilitate trauma access, psychological guidance to facilitate integration should be available before, during, and after administration (Nutt and Carhart-Harris, 2020). This could maximize the positive outcomes while minimizing the negative outcomes. For example, integration support might create the prerequisites for fear extinction and memory reconsolidation, thus erasing the fear memory (Feduccia and Mithoefer, 2018; Inserra, 2018; Nutt and Carhart-Harris, 2020). However, absence of guidance after trauma access may also lead to the reinstauration of trauma and significant distress, highlighting the importance of adequate follow-up.

\section{B. Acute Side Effects Encountered in Uncontrolled Settings}

Psychedelic compounds are considered psychologically and physically safe overall when administered in controlled clinical settings. However, the use of these compounds in uncontrolled clinical settings is potentially less safe because of the absence of trained practitioners and the lack of emergency psychiatric and physical treatments readily available should the need arise. Although no fatalities have been reported after the administration of medical-grade psychedelic compounds in controlled clinical settings, fatalities have been reported after the ingestion of multiple psychoactive substances, including psychedelics, or the ingestion of synthetic phenethylamines such as 4Iodo-2, 5-dimethoxy-N-(2-methoxybenzyl) phenethylamine (25i-NBOMe) (Nichols, 2016; Nichols and Grob, 2018).

Serious psychological side effects that can be encountered in uncontrolled settings after the ingestion of LSD, ayahuasca, DMT, and psilocybin include 1) the experiencing of fear to extents that can be temporarily debilitating and can lead to suicidal or homicidal thought, ideation, or behavior; 2) dangerous and disordered behavior triggered by panic; 3 ) the manifestation or exacerbation of latent psychiatric conditions such as schizophrenia, bipolar disorders, and anxiety; and 4) the instauration of long-lasting perceptual disturbances (Nichols, 2016; Dos Santos et al., 2017; dos Santos et al., 2017). For example, patients with generalized anxiety disorder might undergo particularly challenging and potentially frightening experiences during the administration of the psychedelic brew ayahuasca, which might require pharmacological psychiatric treatment (dos Santos et al., 2017).

Patients diagnosed with acute LSD- and PCPinduced psychoses have been successfully treated with 
the antipsychotic haloperidol (D2 receptor antagonist, 5 $\mathrm{mg}$, i.m.), and chlorpromazine (D1/2/3/5 and $5-\mathrm{HT}_{2 / 6 / 7}$ receptor antagonist, $50 \mathrm{mg}$, i.m.). Haloperidol appears more efficacious in reducing anxiety, conceptual disorganization, and visual hallucinations, whereas chlorpromazine appears more efficacious for somatic and psychological tension (Giannini et al., 1984-1985). However, hallucinogen-induced psychosis is also treated with 5-HT antagonist antipsychotics like olanzapine in emergency settings (Dr. Gobbi, personal communication), even if official guidelines and controlled studies for the use of D2 receptor antagonist and/or 5-HT2 receptor antagonist to treat hallucinogen-induced psychosis are not yet available.

1. Risk of Switch to Mania in Patients with Bipolar Disorder. Another potential side effect that could be encountered after the administration of psychedelic compounds is the risk of switch to mania in patients with BD (Szmulewicz et al., 2015). At least two such case reports are available in the literature. One is of a patient with $\mathrm{BD}$ with a current depressive episode who switched to a manic episode after a 4-day ayahuasca ritual (Szmulewicz et al., 2015). The patient had previously experienced hypomanic episodes, with the most recent occurring 10 days prior to ritual. The man was admitted to hospital and treated with the antipsychotic risperidone and the benzodiazepine clonazepam for 1 month, after which he became asymptomatic and was discharged (Szmulewicz et al., 2015). Another case report is of a male BD type 1 treatment-refractory psychiatrist who had attempted to self-medicate with smoked DMT (up to $1 \mathrm{~g}$ daily), phenelzine (a MAOI inhibitor), and clonazepam and who was admitted to hospital after developing a hypomanic psychotic episode with highly disturbed and agitated behavior 2 to 3 days after interrupting his self-medication schedule (Brown et al., 2017c). These reports suggest the need to assess the likelihood that patients with BD might switch to mania if receiving psychedelic therapy and to identify the most suited antimanic pharmacological approaches to treat such patients should the switch take place.

2. Risk of Psychosis. One of the most significant potential psychological side effects after the ingestion of psychedelic compounds in uncontrolled settings is the onset of a psychotic episode, which can last for several hours or days after the drug has worn off. The most vulnerable patients to the onset of long-lasting psychoses appear to be those who have previously received a diagnosis of SCZ, BD, or personality disorder or patients more inclined to mistrustfulness, fearfulness, and susceptibility on projection as defense (Anastasopoulos and Photiades, 1962; De Gregorio et al., 2016a, 2018; Dos Santos et al., 2017). Other risk factors include mixing other psychoactive substances, such as cannabis. For example, it was reported that an individual with a history of cannabis consumption and hypomania started experiencing psychotic symptoms associated to his hypomanic condition after the inhalation of a cannabis/DMT mix (Umut et al., 2011). Psychotic episodes have also been reported in individuals affiliated with religious groups who ingest ayahuasca for ritualistic and religious purposes, although the incidence appears to overlap that of the general population (dos Santos et al., 2017).

3. Acute Severe Physical Side Effects Encountered in Uncontrolled Settings. From a physiological point of view, psychedelic compounds appear to have a considerable margin of safety. For example, even after the accidental ingestion of very high doses (up to 550 doses) of LSD, no major adverse physiological side effects have been reported in a recent case report of three individuals (Haden and Woods, 2020). On the contrary, positive mental health changes were reported in two out of three patients, such as a 20-year-long reduction of mania with psychotic features in one subject and a reduction in physical pain and morphine addiction in another (Haden and Woods, 2020).

Although rare, conspicuous physiological adverse reactions such as vasoconstriction, coronary artery spasms, and rhabdomyolysis have been reported after the consumption of high doses of psychedelic compounds in unsupervised settings. This may be attributable to the serotonergic action of psychedelics, which affects cardiovascular function and can lead to serotonin syndrome and cardiovascular complications (Berrens et al., 2010; Cogen et al., 1978; Nichols, 2016).

4. Serotonin Syndrome. Another concern surrounding the use of psychedelic compounds in psychiatry is the onset of serotonin syndrome, a potentially lifethreatening condition due to excessive stress on central and peripheral postsynaptic serotonin receptors that results in autonomic and neuromuscular aberrations that can prove fatal (Martin, 1996; Parrott, 2002; Boyer and Shannon, 2005; Scotton et al., 2019). Serotonin syndrome is characterized by changes in mental status including mild agitation; hypervigilance; delirium; autonomic hyperactivity including tachycardia, hyperthermia, hypertension, shivering, and hyperactive bowels; and neuromuscular abnormalities such as hyperreflexia and muscular rigidity (Martin, 1996; Boyer and Shannon, 2005; Scotton et al., 2019). If hyperthermia is not treated, it can lead to more severe side effects, such as rhabdomyolysis, metabolic acidosis, seizures, renal failure, intravascular coagulopathy, and death (Martin, 1996; Boyer and Shannon, 2005; Scotton et al., 2019).

Several approved serotonergic psychiatric drugs, including SSRIs, have been associated with serotonin syndrome, both at clinical doses and after accidental or voluntary overdose (Graudins et al., 1998; Kinzie and Meltzer-Brody, 2005; Paruchuri et al., 2006). A major concern revolves around the ingestion of serotonergic psychedelics by patients who medicate with SSRIs, a combination which can result in serotonin syndrome 
due to synergistic effects of SSRIs and serotonergic psychedelics or other compounds with MAOI activity such as Peganum harmala (Syrian rue) (Bakim et al., 2012). Concerning psychedelics, dissociative anesthetics, and empathogens, serotonin syndrome has been reported after the ingestion of LSD, MDMA, 5methoxydiisopropyltryptamine ("foxy methoxy"), dextromethorphan, and P. harmala and by the combined use of pharmaceutical amphetamines and MDMA, mostly in patients abusing one or more of these substances, and in patients receiving MAOI or SSRI/ SNRI therapies (Silbergeld and Hruska, 1979; Parrott, 2002; Ener et al., 2003; Boyer and Shannon, 2005; Arora and Kannikeswaran, 2010; Bakim et al., 2012; Davies et al., 2014; Tao et al., 2017; Singh et al., 2019).

Although no cases have been reported of serotonin syndrome after the administration of medical-grade psychedelic compounds in clinical settings, this side effect should be taken into consideration. Importantly, the use of SSRIs and SNRIs needs to be carefully taken into consideration, and if required, it should be suspended under medical supervision prior to psychedelic therapy (Bakim et al., 2012). Pharmacogenomic variability might also increase the likelihood of serotonin syndrome, as it was shown for carriers of polymorphisms at the $\mathrm{T} 102 \mathrm{C}$ site of the $5-\mathrm{HT}_{2 \mathrm{~A}}$ receptor (Cooper et al., 2014). This raises the concept that pharmacogenomic screening tests could be implemented for the identification of patients who are suitable to receive psychedelic therapy and those more likely to undergo severe side effects.

5. 3,4-Methylenedioxymethamphetamine Neurotoxicity. Several studies have investigated whether MDMA is neurotoxic on neurotransmitter systems (Schmidt, 1987; Gudelsky et al., 1994; Armstrong and Noguchi, 2004; Granado et al., 2011; Shokry et al., 2019) given that this compound is also used to induce dopaminergic toxicity in mouse models (Blesa and Przedborski, 2014). Catechols derived from MDMA metabolism appear to be responsible for the neurotoxic effects of MDMA given that direct intracerebroventricular administration of MDMA does not elicit 5-HT neurotoxicity (Green et al., 2003). For example, intrastriatal administration of 2,4,5-trihydroxymethamphetamine significantly depletes both 5-HT and DA, intracortical administration decreases 5-HT, and intracerebroventricular administration moderately depletes striatal DA without affecting 5-HT levels (Johnson et al., 1992; Zhao et al., 1992). Other metabolites, such as 6-HO-MDMA, appear to be nontoxic given that intrastriatal and intracerebroventricular administration does not affect 5-HT or DA levels (Zhao et al., 1992). Further metabolites that might contribute to the neurotoxic properties of MDMA are quinone-thioethers, orto-quinones, and the glutathione conjugates 5-GSyl- $\alpha$-MeDA and 2,5-bis-(glutathion-S-yl)- $\alpha$-methyldopamine (2,5-bis-(glutathione-Syl)- $\alpha$-MeDA) (Monks et al., 2001; Green et al., 2003).
Acute intracerebroventricular injections of 5-GSyl$\alpha$-MeDA were reported to acutely increase DA and 5HT levels and to increase long-term DA (but not 5-HT) turnover (Miller et al., 1996). Multiple intrastriatal and intracortical administrations of this metabolite significantly deplete striatal and cortical (but not hippocampal) 5-HT (Bai et al., 1999). 2,5-Bis-(glutathion-S-yl)- $\alpha$-MeDA administration was shown to reduce cortical 5-HT and hippocampal 5-HT and 5hydroxy-indoleacetic acid, to modestly reduce striatal 5-HT concentrations, and to produce 5-HT neurotoxicity, which was limited to the terminal areas, without affecting DA concentrations or those of its metabolites (Miller et al., 1997). Similarly, intrastriatal and intracortical administration of 2,5-bis-(glutathion-S-yl)$\alpha$-MeDA significantly depletes striatal, cortical, and hippocampal 5-HT (Bai et al., 1999). However, in many toxicity studies, the administration is often repeated, and higher doses are employed (i.e., $5-20 \mathrm{mg} / \mathrm{kg}$ ) compared with clinical trials (Pantoni and Anagnostaras, 2019). However, when taking into consideration clinical or recreational doses (i.e., $1-2 \mathrm{mg} / \mathrm{kg}$ ), no cognitive impairments or long-term neurotoxicity is observable (Pantoni and Anagnostaras, 2019).

\section{Abuse Potential and Potential Long-Term Side Effects of Psychedelic Compounds}

1. Codependence and Psychosis. Several psychoses induced by psychedelics are linked to the codependence with other substances, especially cannabis and alcohol. Even if psychosis induced by multiple substances is a frequent occurrence in the psychiatric emergency departments, there are only a few studies and case reports available (Warren et al., 2013; Paterson et al., 2015; Dos Santos et al., 2017).

2. Abuse Potential. The abuse potential profile of psychedelic compounds is weak, and so is the recurrence of significant psychiatric sequelae from the administration of psychedelic compounds in controlled clinical settings (Nichols, 2016). Studies in animals require more deep investigations (see below). A rare case of physical dependence from, and tolerance to, LSD has recently been reported in the literature (Modak et al., 2019). One recent study investigated the abuse liability of low- and high-dose LSD, psilocybin, and mescaline and repeated LSD administration via the intracranial self-stimulation paradigm in rats, which is widely adopted to determine the abuse potential of a putative drug (Sakloth et al., 2019). Interestingly, the acute administration of LSD, psilocybin, and mescaline fails to produce reliable evidence of abuse (Sakloth et al., 2019). Moreover, the authors found that repeated LSD (at the highest doses tested, but not at low doses) tends to attenuate the rewarding effects of the psychostimulant methamphetamine and the depressant $\kappa$-opioid receptor agonist U69,593, although only findings for the latter were statistically significant (Sakloth et al., 2019). 
In another study, no preference for the self-administration of DMT or psilocybin was observed in comparison with saline (aside from one subject showing transient higher rates of DMT and psilocybin self-administration), and none self-administered DOI (Fantegrossi et al., 2004). On the other hand, in nonhuman primates, minimal rates of LSD self-injection were observed after a daily access procedure, but a more substantial self-administration was observed after an intermittent access procedure (Goodwin, 2016). Therefore, although it seems that these compounds present low abuse liability, more adequately powered studies are required to confirm these findings, especially those using different doses and different regimens of psychedelics.

3. Hallucinogen-Persisting Perception Disorder. Hallucinogen-persisting perception disorder is the reoccurrence of drug-like effects long after the substance has worn out (Orsolini et al., 2017; Halpern et al., 2018; Knuijver et al., 2018; Skryabin et al., 2018). Although the incidence of this condition is low and seldom impairs life quality significantly, it has been reported in patients with alcoholism (Batzer et al., 1999), military personnel (Stanton and Bardoni, 1972), and individuals with a longterm history of psychedelic use/abuse proportionally to the number of times the substance was ingested but sometimes after one administration (Batzer et al., 1999; Orsolini et al., 2017; Anderson et al., 2018; Halpern et al., 2018; Knuijver et al., 2018; Martinotti et al., 2018; Kurtom et al., 2019; Skryabin et al., 2018; Goldman et al., 2007). Successful treatment of hallucinogen-persisting perception disorder has been reported with high-potency serotonergic benzodiazepines such as clonazepam (Lerner et al., 2003).

\section{Potential Long-Term Physical Side Effects in Uncontrolled Settings}

1. Cardiovascular Side Effects. Other potential severe side effects that might arise after microdosing concern the direct repeated stimulation of cardiac 5$\mathrm{HT}_{2 \mathrm{~B}}$ receptors by psychedelics, as well as the indirect $5-\mathrm{HT}_{2 \mathrm{~B}}$ receptors stimulation due to increased circulating 5-HT levels (Connolly et al., 1997; Fishman, 1999). Cardiac $5-\mathrm{HT}_{2 \mathrm{~B}}$ receptor overstimulation can lead to cardiovascular dysfunction after significant histologic and functional changes to the heart valves (Gustafsson et al., 2005; Elangbam et al., 2008; Hutcheson et al., 2011). For example, valvular fibrosis leading to valvular thickening and valvular regurgitation were observed in patients receiving anorectic (appetite-depressing) drugs with $5-\mathrm{HT}_{2 \mathrm{~A} / 2 \mathrm{~B}}$ receptor action, such as the fenfluraminephentermine combination (Connolly et al., 1997; Curfman, 1997; Hutcheson et al., 2011). Animal models of repeated 5-HT administration confirmed those findings and added that these effects are accompanied by increased cardiac $5-\mathrm{HT}_{2 \mathrm{~B}}$ receptor and decreased SERT expression (Gustafsson et al., 2005; Elangbam et al., 2008; Hutcheson et al., 2011). Similarly, the ergot alkaloids ergotamine and methysergide, which are used for the treatment of migraine and cluster headaches, were found to damage valvular function to extents that may require valve replacement due to pathologic fibrosis and thickening (Graham et al., 1966; Graham, 1967; Mason et al., 1977; Redfield et al., 1992). Pulmonary hypertension is another risk that should be assessed in response to the repeated administration of serotonergic psychedelics. This severe side effect was observed after the repeated administration of fenfluramine and dexfenfluramine, a postsynaptic 5-HT releaser and presynaptic SERT inhibitor, respectively, and aminorex, which is an NE/catecholaminergic releaser (Gurtner, 1985; Fishman, 1999; Gaine et al., 2000).

Severe cardiovascular side effects (cardiopulmonary arrest) were reported in one individual after frequent ingestion of Psilocybe semilanceata mushrooms for 1 month (Borowiak et al., 1998). Although this represents an extremely rare case report, future efforts should be directed toward identifying potential cardiac effects that might result from the repeated administration of psychedelic compounds, even in microdoses. Further efforts should be devoted to identifying biological biomarkers and psychological and neuroimaging predictors of psychedelic-induced subjective side effects. Further efforts should be devoted to identifying biological biomarkers and psychological predictors of psychedelicinduced subjective effects (such as rostral ACC thickness), as well as dissociation, negative experiences, and psychoses. This could help identify patients more likely to encounter challenging experiences (such as those with high neuroticism, emotional instability, and family history of psychosis, bipolar disorders, and childhood trauma) and implement adequate strategies to maximize therapeutic improvement while minimizing potential biological and psychological short- and longterm side effects (Barrett et al., 2017; Thal et al., 2019; Lewis et al., 2020).

\section{Recommendations for Future Research}

Clinically administered psychedelics elicit rapid, robust, and sustained antidepressant and anxiolytic effects with no major apparent negative outcomes. Psychedelics modulate pathways involved in the improvement and remission of psychiatric disorders. Some pathways overlap those activated by currently available psychiatric drugs, whereas others are psychedelicspecific and result from biased signaling. From a psychiatric perspective, the most relevant transcriptional programs induced span neuroplasticity, neuroimmunomodulation, and serotonergic, glutamatergic, dopaminergic, and GABAergic systems modulation. Future studies should dissect transcriptional outcomes in a compound- and dosage-specific fashion. Acute and long-term positive and negative effects on neurotransmission should be investigated. Long-term neurobiological 
side effects need to be systematically assessed after acute and repeated administration of psychedelics. Although the profound effects of psychedelics on immune processes appear to be mostly modulatory and protective, host immunocompetency needs to be thoroughly assessed, especially should repeated administration be needed to achieve remission.

Given that microdosing is gaining momentum as a potential alternative therapeutic strategy for medical, psychiatric, and creativity-enhancing purposes, larger RCTs could investigate whether microdosing can elicit clinical improvement and/or remission of psychiatric symptoms. Whether long-term microdosing regimens would be necessary to reach remission, studies should investigate the sequelae, if any, of this posology. Future studies should aim at identifying psychological and biological predictors of positive (and negative) outcomes to screen patients who are more likely to encounter challenging experiences, such as those of young age, of high emotional excitability, and who do not feel ready (Studerus et al., 2012; Haijen et al., 2018). Pharmacogenomics (Evans and McLeod, 2003; Whirl-Carrillo et al., 2012) and pharmacoepigenomics (Peedicayil, 2019) could be applied in this endeavor to address biological variability underlying different responses to psychedelics, as previously done for other psychiatric compounds (Yamanouchi et al., 2003; Abdolmaleky et al., 2008). For example, a recent study found that carriers of the D1 receptor polymorphism rs11133767 (two $\mathrm{T}$ alleles) had disproportionally engaged in many more illicit drug use episodes compared with the carriers of the C allele (Vizeli and Liechti, 2019). Similar studies could identify polymorphisms that increase the likelihood of encountering challenging experiences or of abusing psychedelics.

Whether certain individuals are identified who have an increased likelihood of undergoing challenging experiences, greater psychophysical preparation should be required, as well as enhanced psychological support before, during, and after the treatment. If psychedelics will be approved as therapeutics, whether certain individuals should be excluded from accessing this type of treatment will be determined in phase III clinical trials. So far, in clinical studies, this has been the case given that schizophrenic and/or bipolar individuals may have an increased likelihood of drug-induced psychosis or drug-induced manic or depressive episodes. Another main challenge of research is to design a novel selective $5-\mathrm{HT}_{2 \mathrm{~A}}$ receptor agonist with enhanced therapeutic potential and with low affinity for D2 and TAAR1 receptors that could be responsible for the propsychotic and drug-rewarding side effects.

Psychedelics might also prove to be useful for other neuroinflammatory and systemic conditions that may or may not be comorbid with psychiatric disorders. For example, preclinical efficacy has been shown in inflammatory conditions such as high-fat diet-induced obesity and diabetes (Flanagan et al., 2019b), gut inflammatory challenges (Nau et al., 2013, 2015), and asthma (Flanagan et al., 2019a). Possible utility in other psychiatric conditions, such as eating disorders, has been hypothesized and should be further explored (Foldi et al., 2020).

Ultimately, the knowledge generated by ongoing clinical studies may boost the acceptance of this approach by legislative, funding, and academic bodies, leading to the implementation of the necessary policy shifts to allow this therapy to reach the bedside. Lastly, compounds with structural analogy might represent an alternative to natural and semisynthetic psychedelics in an attempt to decouple the therapeutic from psychedelic effects of these compounds (McLean et al., 2006; Zanos et al., 2017; Harvey et al., 2019; Kenakin, 2019). For example, 2-([2-(4-cyano-2,5-dimethoxyphenyl)ethylamino]methyl)phenol, a novel and synthesized derivate of $N$-benzyl-substituted phenethylamine hallucinogens, displays strong binding affinity and selectivity for the $5 \mathrm{HT}_{2 \mathrm{~A}}$ receptor (Hansen et al., 2014; Jensen et al., 2020). In vivo studies revealed that this compound was able to induce behavioral effects (e.g., inducing headtwitch response) with less extent compared with the $5-\mathrm{HT}_{2 \mathrm{~A} / 2 \mathrm{c}}$ receptor agonist DOI (Fantegrossi et al., 2015), thus suggesting a different behavioral profile of this compound despite the high selectivity for the $5 \mathrm{HT}_{2 \mathrm{~A}}$ receptor. Moreover, 1-acetyl-LSD (ALD-52), a derivative of LSD, has been demonstrated to produce psychedelic effects in humans, as suggested by older studies (Rothlin, 1957; Malitz et al., 1960). Recently, several other 1acyl-substituted LSD derivatives, including 1-propanoyl-LSD (1P-LSD) and 1-butanoyl-LSD (1B-LSD), have appeared as recreational drugs (Brandt et al., 2017). A recent study investigated the effects of these two compounds in mice, revealing that, although LSD acts as an agonist at $5-\mathrm{HT}_{2 \mathrm{~A}}$ receptors, $\mathrm{ALD}-52,1 \mathrm{P}-\mathrm{LSD}$, and $1 \mathrm{~B}-$ LSD display weak affinity even though they induce head twitches (a preclinical index of psychedelic effect) in mice with relatively high potency (Halberstadt et al., 2020). In addition, LSD was detected in the plasma of rats after administration of ALD-52 and 1P-LSD, thus suggesting that these compounds are deacylated in vivo. Therefore, ALD-52, 1P-LSD, and 1B-LSD can be considered as prodrugs for LSD. For details, please see Halberstadt et al. (2020). Future studies should investigate the translational potential of these analogs and their effects in humans.

\section{Conclusion}

Together, given the available evidence to date pointing toward safety, tolerability, and efficacy of psychedelic compounds as potential novel therapeutics in psychiatry, we conclude that psychedelics are challenging the current paradigm in psychiatry. If 1) larger RCTs will corroborate these preliminary findings and 2) the acute and long-term side effect profiles will be 
similar, or better, than those of existing psychiatric drugs, several psychedelic compounds may be soon developed in clinical trials and eventually be approved by pharmaceutical regulatory governmental authorities.

\section{Acknowledgments}

We thank Dr. Franco Recchia and Photo Grafic (www.photo-grafic. it) for help with the preparation of the figures.

\section{Authorship Contributions}

Participated in research design: Inserra, De Gregorio, Gobbi.

Wrote or contributed to the writing of the manuscript: Inserra, De Gregorio, Gobbi.

\section{References}

Aalto S, Hirvonen J, Kajander J, Scheinin H, Någren K, Vilkman H, Gustafsson L, Syvälahti E, and Hietala J (2002) Ketamine does not decrease striatal dopamine D2 receptor binding in man. Psychopharmacology (Berl) 164:401-406.

aan het Rot M, Collins KA, Murrough JW, Perez AM, Reich DL, Charney DS, and Mathew SJ (2010) Safety and efficacy of repeated-dose intravenous ketamine for treatment-resistant depression. Biol Psychiatry 67:139-145.

Abdallah CG, Sanacora G, Duman RS, and Krystal JH (2015) Ketamine and rapidacting antidepressants: a window into a new neurobiology for mood disorder therapeutics. Annu Rev Med 66:509-523.

Abdolmaleky HM, Zhou JR, Thiagalingam S, and Smith CL (2008) Epigenetic and pharmacoepigenomic studies of major psychoses and potentials for therapeutics. Pharmacogenomics 9:1809-1823.

Abel T, Havekes R, Saletin JM, and Walker MP (2013) Sleep, plasticity and memory from molecules to whole-brain networks. Curr Biol 23:R774-R788.

Abi-Saab WM, Bubser M, Roth RH, and Deutch AY (1999) 5-HT2 receptor regulation of extracellular GABA levels in the prefrontal cortex. Neuropsychopharmacology 20:92-96.

Abramson HA (1955) Lysergic acid diethylamide (LSD-25): III. As an adjunct to psychotherapy with elimination of fear of homosexuality. J Psychol 39:127-155.

Abramson HA and Jarvik ME (1955) Lysergic acid diethylamide (LSD-25): IX. Effect on snails. J Psychol 40:337-340.

Adams JD Jr, Baillie TA, Trevor AJ, and Castagnoli N Jr (1981) Studies on the biotransformation of ketamine. 1-Identification of metabolites produced in vitro from rat liver microsomal preparations. Biomed Mass Spectrom 8:527-538.

Adams JD, Castagnoli N, and Trevor AJ (1978) Quantitative analysis of ketamine enantiomers. Proc West Pharmacol Soc 21:471-472.

Aday JS, Bloesch EK, and Davoli CC (2020) Can psychedelic drugs attenuate agerelated changes in cognition and affect?. J Cogn Enhanc 4:219-227.

Adell A and Artigas F (1991) Differential effects of clomipramine given locally or systemically on extracellular 5-hydroxytryptamine in raphe nuclei and frontal cortex. An in vivo brain microdialysis study. Naunyn Schmiedebergs Arch Pharmacol 343:237-244.

Aghajanian GK (1980) Mescaline and LSD facilitate the activation of locus coeruleus neurons by peripheral stimuli. Brain Res 186:492-498.

Aghajanian GK (2009) Modeling "psychosis" in vitro by inducing disordered neuronal network activity in cortical brain slices. Psychopharmacology (Berl) 206: $575-585$.

Aghajanian GK, Foote WE, and Sheard MH (1968) Lysergic acid diethylamide: sensitive neuronal units in the midbrain raphe. Science 161:706-708.

Aghajanian GK, Foote WE, and Sheard MH (1970) Action of psychotogenic drugs on single midbrain raphe neurons. J Pharmacol Exp Ther 171:178-187.

Aghajanian GK and Hailgler HJ (1975) Hallucinogenic indoleamines: preferential action upon presynaptic serotonin receptors. Psychopharmacol Commun 1: 619-629.

Aghajanian GK and Marek GJ (1997) Serotonin induces excitatory postsynaptic potentials in apical dendrites of neocortical pyramidal cells. Neuropharmacology 36:589-599.

Aghajanian GK and Marek GJ (1999) Serotonin, via 5-HT2A receptors, increases EPSCs in layer V pyramidal cells of prefrontal cortex by an asynchronous mode of glutamate release. Brain Res 825:161-171.

Aghajanian GK and Vandermaelen CP (1982) Intracellular recordings from serotonergic dorsal raphe neurons: pacemaker potentials and the effect of LSD. Brain Res 238:463-469.

Ago Y, Tanabe W, Higuchi M, Tsukada S, Tanaka T, Yamaguchi T, Igarashi H, Yokoyama R, Seiriki K, Kasai A, et al. (2019) (R)-Ketamine induces a greater increase in prefrontal 5-HT release than (S)-Ketamine and ketamine metabolites via an AMPA receptor-independent mechanism. Int $J$ Neuropsychopharmacol 22: $665-674$.

Aguirre N, Ballaz S, Lasheras B, and Del Río J (1998) MDMA ('Ecstasy') enhances 5HT1A receptor density and 8-OH-DPAT-induced hypothermia: blockade by drugs preventing 5-hydroxytryptamine depletion. Eur J Pharmacol 346:181-188.

Ahern GP (2011) 5-HT and the immune system. Curr Opin Pharmacol 11:29-33.

Albizu L, Holloway T, González-Maeso J, and Sealfon SC (2011) Functional crosstalk and heteromerization of serotonin 5-HT2A and dopamine D2 receptors. Neuropharmacology 61:770-777.

Alboni S, Poggini S, Garofalo S, Milior G, El Hajj H, Lecours C, Girard I, Gagnon S, Boisjoly-Villeneuve S, Brunello N, et al. (2016) Fluoxetine treatment affects the inflammatory response and microglial function according to the quality of the living environment. Brain Behav Immun 58:261-271.

Albott CS, Lim KO, Forbes MK, Erbes C, Tye SJ, Grabowski JG, Thuras P, Batres-YCarr TM, Wels J, and Shiroma PR (2018) Efficacy, safety, and durability of repeated ketamine infusions for comorbid posttraumatic stress disorder and treatment-resistant depression. J Clin Psychiatry 79:17m11634.

Alcocer-Gómez E, de Miguel M, Casas-Barquero N, Núñez-Vasco J, Sánchez-Alcazar JA, Fernández-Rodríguez A, and Cordero MD (2014) NLRP3 inflammasome is activated in mononuclear blood cells from patients with major depressive disorder. Brain Behav Immun 36:111-117.

Aleksandrova LR, Phillips AG, and Wang YT (2017) Antidepressant effects of ketamine and the roles of AMPA glutamate receptors and other mechanisms beyond NMDA receptor antagonism. J Psychiatry Neurosci 42:222-229.

Alhaider AA, Hamon M, and Wilcox GL (1993) Intrathecal 5-methoxy-N,N-dimethyltryptamine in mice modulates 5 -HT1 and 5-HT3 receptors. Eur $J$ Pharmacol 249:151-160.

Alkire MT, Hudetz AG, and Tononi G (2008) Consciousness and anesthesia. Science 322: $876-880$.

Alt A, Nisenbaum ES, Bleakman D, and Witkin JM (2006a) A role for AMPA receptors in mood disorders. Biochem Pharmacol 71:1273-1288.

Alt A, Weiss B, Ogden AM, Li X, Gleason SD, Calligaro DO, Bleakman D, and Witkin JM (2006b) In vitro and in vivo studies in rats with LY293558 suggest AMPA/ kainate receptor blockade as a novel potential mechanism for the therapeutic treatment of anxiety disorders. Psychopharmacology (Berl) 185:240-247.

Alt A, Witkin JM, and Bleakman D (2005) AMPA receptor potentiators as novel antidepressants. Curr Pharm Des 11:1511-1527.

Amargós-Bosch M, López-Gil X, Artigas F, and Adell A (2006) Clozapine and olanzapine, but not haloperidol, suppress serotonin efflux in the medial prefrontal cortex elicited by phencyclidine and ketamine. Int $J$ Neuropsychopharmacol 9:565-573.

Amoroso T (2019) The spurious relationship between ecstasy use and neurocognitive deficits: a Bradford Hill review. Int J Drug Policy 64:47-53.

Ampuero E, Rubio FJ, Falcon R, Sandoval M, Diaz-Veliz G, Gonzalez RE, Earle N, Dagnino-Subiabre A, Aboitiz F, Orrego F, et al. (2010) Chronic fluoxetine treatment induces structural plasticity and selective changes in glutamate receptor subunits in the rat cerebral cortex. Neuroscience 169:98-108.

Anacker C, Zunszain PA, Cattaneo A, Carvalho LA, Garabedian MJ, Thuret S, Price J, and Pariante CM (2011) Antidepressants increase human hippocampal neurogenesis by activating the glucocorticoid receptor. Mol Psychiatry 16:738-750.

Anand A, Li Y, Wang Y, Lowe MJ, and Dzemidzic M (2009) Resting state corticolimbic connectivity abnormalities in unmedicated bipolar disorder and unipolar depression. Psychiatry Res 171:189-198.

Anastasopoulos G and Photiades H (1962) Effects of LSD-25 on relatives of schizophrenic patients. J Mental Science 108:95-98.

Andén NE, Corrodi H, Fuxe K, and Hökfelt T (1968) Evidence for a central 5hydroxytryptamine receptor stimulation by lysergic acid diethylamide. $\mathrm{Br}$ J Pharmacol 34:1-7.

Anderson L, Lake H, and Walterfang M (2018) The trip of a lifetime: hallucinogen persisting perceptual disorder. Australas Psychiatry 26:11-12.

Anderson PM, Jones NC, O'Brien TJ, and Pinault D (2017) The N-methyl d-aspartate glutamate receptor antagonist ketamine disrupts the functional state of the corticothalamic pathway. Cereb Cortex 27:3172-3185.

Anderson T, Petranker R, Christopher A, Rosenbaum D, Weissman C, Dinh-Williams LA, Hui K, and Hapke E (2019) Psychedelic microdosing benefits and challenges: an empirical codebook. Harm Reduct $J$ 16:43.

Andersson M, Persson M, and Kjellgren A (2017) Psychoactive substances as a last resort-a qualitative study of self-treatment of migraine and cluster headaches. Harm Reduct $J$ 14:60.

Andrews-Hanna JR (2012) The brain's default network and its adaptive role in internal mentation. Neuroscientist 18:251-270.

Angelucci F, Ricci V, Gelfo F, Martinotti G, Brunetti M, Sepede G, Signorelli M, Aguglia E, Pettorruso M, Vellante F, et al. (2014) BDNF serum levels in subjects developing or not post-traumatic stress disorder after trauma exposure. Brain Cogn 84:118-122.

Angers S, Salahpour A, and Bouvier M (2002) Dimerization: an emerging concept for $\mathrm{G}$ protein-coupled receptor ontogeny and function. Annu Rev Pharmacol Toxicol 42: 409-435.

Anis NA, Berry SC, Burton NR, and Lodge D (1983) The dissociative anaesthetics, ketamine and phencyclidine, selectively reduce excitation of central mammalian neurones by N-methyl-aspartate. Br J Pharmacol 79:565-575.

Anneken JH, Cunningham JI, Collins SA, Yamamoto BK, and Gudelsky GA (2013) MDMA increases glutamate release and reduces parvalbumin-positive GABAergic cells in the dorsal hippocampus of the rat: role of cyclooxygenase. J Neuroimmune Pharmacol 8:58-65.

Anneken JH and Gudelsky GA (2012) MDMA produces a delayed and sustained increase in the extracellular concentration of glutamate in the rat hippocampus. Neuropharmacology 63:1022-1027.

Antonini V, Prezzavento O, Coradazzi M, Marrazzo A, Ronsisvalle S, Arena E, and Leanza G (2009) Anti-amnesic properties of (+/-)-PPCC, a novel sigma receptor ligand, on cognitive dysfunction induced by selective cholinergic lesion in rats. $J$ Neurochem 109:744-754.

Appadu BL and Lambert DG (1996) Interaction of i.v. anaesthetic agents with 5-HT3 receptors. Br J Anaesth 76:271-273.

Archer T, Minor BG, and Post C (1985) Blockade and reversal of 5-methoxy-N,Ndimethyltryptamine-induced analgesia following noradrenaline depletion. Brain Res 333:55-61.

Argento E, Strathdee SA, Tupper K, Braschel M, Wood E, and Shannon K (2017) Does psychedelic drug use reduce risk of suicidality? Evidence from a longitudinal community-based cohort of marginalised women in a Canadian setting. BMJ Open 7:e016025.

Argento E, Tupper KW, and Socias ME (2019) The tripping point: the potential role of psychedelic-assisted therapy in the response to the opioid crisis. Int J Drug Policy 66:80-81. 
Armstrong BD and Noguchi KK (2004) The neurotoxic effects of 3,4-methylenedioxymethamphetamine (MDMA) and methamphetamine on serotonin, dopamine, and GABA-ergic terminals: an in-vitro autoradiographic study in rats. Neurotoxicology 25:905-914.

Aronson CE and Hanno ER (1978) Effects of ketamine on the isolated perfused rat heart. Gen Pharmacol 9:249-255.

Arora B and Kannikeswaran N (2010) The serotonin syndrome-the need for physician's awareness. Int $J$ Emerg Med 3:373-377.

Artigas F, Romero L, de Montigny C, and Blier P (1996) Acceleration of the effect of selected antidepressant drugs in major depression by 5 -HT1A antagonists. Trends Neurosci 19:378-383.

Asanuma C (1992) Noradrenergic innervation of the thalamic reticular nucleus: a light and electron microscopic immunohistochemical study in rats. J Comp Neurol 319:299-311.

Aston-Jones G and Bloom FE (1981) Activity of norepinephrine-containing locus coeruleus neurons in behaving rats anticipates fluctuations in the sleep-waking cycle. J Neurosci 1:876-886.

Aston-Jones G and Cohen JD (2005) An integrative theory of locus coeruleusnorepinephrine function: adaptive gain and optimal performance. Annu Rev Neurosci 28:403-450.

Aston-Jones G, Rajkowski J, and Cohen J (1999) Role of locus coeruleus in attention and behavioral flexibility. Biol Psychiatry 46:1309-1320.

Austin MC, Whitehead RE, Edgar CL, Janosky JE, and Lewis DA (2002) Localized decrease in serotonin transporter-immunoreactive axons in the prefrontal cortex of depressed subjects committing suicide. Neuroscience 114:807-815.

Autry AE, Adachi M, Nosyreva E, Na ES, Los MF, Cheng PF, Kavalali ET, and Monteggia LM (2011) NMDA receptor blockade at rest triggers rapid behavioural antidepressant responses. Nature 475:91-95.

Aydemir O, Deveci A, and Taneli F (2005) The effect of chronic antidepressant treatment on serum brain-derived neurotrophic factor levels in depressed patients: a preliminary study. Prog Neuropsychopharmacol Biol Psychiatry 29 $261-265$

Azzam AAH, McDonald J, and Lambert DG (2019) Hot topics in opioid pharmacology: mixed and biased opioids. Br J Anaesth 122:e136-e145.

Backstrom JR, Chang MS, Chu H, Niswender CM, and Sanders-Bush E (1999) Agonist-directed signaling of serotonin 5-HT2C receptors: differences between serotonin and lysergic acid diethylamide (LSD). Neuropsychopharmacology 21 (2 Suppl):77S-81S.

Baganz NL and Blakely RD (2013) A dialogue between the immune system and brain, spoken in the language of serotonin. ACS Chem Neurosci 4:48-63.

Bahji A, Forsyth A, Groll D, and Hawken ER (2020) Efficacy of 3,4-methylenedioxymethamphetamine (MDMA)-assisted psychotherapy for posttraumatic stress disorder: a systematic review and meta-analysis. Prog Neuropsychopharmacol Biol Psychiatry 96:109735.

Bai F, Lau SS, and Monks TJ (1999) Glutathione and N-acetylcysteine conjugates of $\alpha$-methyldopamine produce serotonergic neurotoxicity: possible role in methylenedioxyamphetamine-mediated neurotoxicity. Chem Res Toxicol 12: 1150-1157.

Bakim B, Sertcelik S, and Tankaya O (2012) A case of serotonin syndrome with antidepressant treatment and concomitant use of the herbal remedy (peganum harmala). Klinik Psikofarmakol BBülteni 22:359-361.

Balasuriya D, D'Sa L, Talker R, Dupuis E, Maurin F, Martin P, Borgese F, Soriani O, and Edwardson JM (2014) A direct interaction between the sigma-1 receptor and the hERG voltage-gated K+ channel revealed by atomic force microscopy and homogeneous time-resolved fluorescence (HTRF®). J Biol Chem 289:32353-32363.

Ball JR and Armstrong JJ (1961) The use of L.S.D. 25 (D-lysergic acid diethylamide) in the treatment of the sexual perversions. Can Psychiatr Assoc J 6:231-235.

Bandelow B, Zohar J, Hollander E, Kasper S, Möller H-J, Zohar J, Hollander E, Kasper S, Möller HJ, Bandelow B, et al.; WFSBP Task Force on Treatment Guidelines for Anxiety, Obsessive-Compulsive and Post-Traumatic Stress Disoders (2008) World Federation of Societies of Biological Psychiatry (WFSBP) guidelines for the pharmacological treatment of anxiety, obsessive-compulsive and posttraumatic stress disorders - first revision. World $J$ Biol Psychiatry 9:248-312.

Bangasser DA, Reyes BA, Piel D, Garachh V, Zhang XY, Plona ZM, Van Bockstaele EJ, Beck SG, and Valentino RJ (2013) Increased vulnerability of the brain norepinephrine system of females to corticotropin-releasing factor overexpression. $\mathrm{Mol}$ Psychiatry 18:166-173.

Bangasser DA, Wiersielis KR, and Khantsis S (2016) Sex differences in the locus coeruleus-norepinephrine system and its regulation by stress. Brain Res 1641 177-188.

Banks WA (2005) Blood-brain barrier transport of cytokines: a mechanism for neuropathology. Curr Pharm Des 11:973-984.

Bankson MG and Cunningham KA (2001) 3,4-Methylenedioxymethamphetamine (MDMA) as a unique model of serotonin receptor function and serotonin-dopamine interactions. $J$ Pharmacol Exp Ther 297:846-852.

Bankson MG and Yamamoto BK (2004) Serotonin-GABA interactions modulate MDMA-induced mesolimbic dopamine release. J Neurochem 91:852-859.

Barbanoj MJ, Riba J, Clos S, Giménez S, Grasa E, and Romero S (2008) Daytime Ayahuasca administration modulates REM and slow-wave sleep in healthy volunteers. Psychopharmacology (Berl) 196:315-326.

Barbosa PC, Strassman RJ, da Silveira DX, Areco K, Hoy R, Pommy J, Thoma R, and Bogenschutz M (2016) Psychological and neuropsychological assessment of regular hoasca users. Compr Psychiatry 71:95-105.

Barchas JD and Freedman DX (1963) Brain amines: response to physiological stress. Biochem Pharmacol 12:1232-1235.

Barnett L, Muthukumaraswamy SD, Carhart-Harris RL, and Seth AK (2020) Decreased directed functional connectivity in the psychedelic state. Neuroimage $\mathbf{2 0 9}$ 116462.

Barrett FS, Carbonaro TM, Hurwitz E, Johnson MW, and Griffiths RR (2018) Doubleblind comparison of the two hallucinogens psilocybin and dextromethorphan: effects on cognition. Psychopharmacology (Berl) 235:2915-2927.
Barrett FS, Doss MK, Sepeda ND, Pekar JJ, and Griffiths RR (2020a) Emotions and brain function are altered up to one month after a single high dose of psilocybin. $S c i$ Rep 10:2214.

Barrett FS and Griffiths RR (2018) Classic hallucinogens and mystical experiences: phenomenology and neural correlates. Curr Top Behav Neurosci 36:393-430.

Barrett FS, Johnson MW, and Griffiths RR (2017) Neuroticism is associated with challenging experiences with psilocybin mushrooms. Pers Individ Dif 117:155-160.

Barrett FS, Krimmel SR, Griffiths RR, Seminowicz DA, and Mathur BN (2020b) Psilocybin acutely alters the functional connectivity of the claustrum with brain networks that support perception, memory, and attention. Neuroimage 218:116980.

Barrot M (2012) Tests and models of nociception and pain in rodents. Neuroscience 211:39-50.

Basso L, Bönke L, Aust S, Gärtner M, Heuser-Collier I, Otte C, Wingenfeld K, Bajbouj $\mathrm{M}$, and Grimm S (2020) Antidepressant and neurocognitive effects of serial ketamine administration versus ECT in depressed patients. J Psychiatr Res 123:1-8.

Battaglia G, Brooks BP, Kulsakdinun C, and De Souza EB (1988) Pharmacologic profile of MDMA (3,4-methylenedioxymethamphetamine) at various brain recognition sites. Eur J Pharmacol 149:159-163.

Batzer W, Ditzler T, and Brown C (1999) LSD use and flashbacks in alcoholic patients. J Addict Dis 18:57-63.

Baudry A, Pietri M, Launay J-M, Kellermann O, and Schneider B (2019) Multifaceted regulations of the serotonin transporter: impact on antidepressant response. Front Neurosci 13:91.

Bauer M, Whybrow PC, Angst J, Versiani M, and Möller H-J; World Federation of Societies Biological Psychiatry Task Force on Treatment Guidelines for Unipolar Depressive Disorders (2002) World Federation of Societies of Biological Psychiatry (WFSBP) Guidelines for Biological Treatment of Unipolar Depressive Disorders, Part 1: acute and continuation treatment of major depressive disorder. World $J$ Biol Psychiatry 3:5-43.

Baumann MH, Wang X, and Rothman RB (2007) 3,4-Methylenedioxymethamphetamine (MDMA) neurotoxicity in rats: a reappraisal of past and present findings. Psychopharmacology (Berl) 189:407-424.

Baumann P (1996) Pharmacology and pharmacokinetics of citalopram and other SSRIs. Int Clin Psychopharmacol 11 (Suppl 1):5-11.

Baumeister D, Akhtar R, Ciufolini S, Pariante CM, and Mondelli V (2016) Childhood trauma and adulthood inflammation: a meta-analysis of peripheral C-reactive protein, interleukin-6 and tumour necrosis factor- $\alpha$. Mol Psychiatry 21:642-649.

Bazhenov M, Timofeev I, Steriade M, and Sejnowski TJ (1999) Self-sustained rhythmic activity in the thalamic reticular nucleus mediated by depolarizing GABAA receptor potentials. Nat Neurosci 2:168-174.

Beggiato S, Borelli AC, Borroto-Escuela D, Corbucci I, Tomasini MC, Marti M, Antonelli T, Tanganelli S, Fuxe K, and Ferraro L (2017) Cocaine modulates allosteric $\mathrm{D}_{2}-\sigma_{1}$ receptor-receptor interactions on dopamine and glutamate nerve terminals from rat striatum. Cell Signal 40:116-124.

Behrens MM, Ali SS, Dao DN, Lucero J, Shekhtman G, Quick KL, and Dugan LL (2007) Ketamine-induced loss of phenotype of fast-spiking interneurons is mediated by NADPH-oxidase. Science 318:1645-1647.

Béique JC, Imad M, Mladenovic L, Gingrich JA, and Andrade R (2007) Mechanism of the 5-hydroxytryptamine $2 \mathrm{~A}$ receptor-mediated facilitation of synaptic activity in prefrontal cortex. Proc Natl Acad Sci USA 104:9870-9875.

Belforte JE, Zsiros V, Sklar ER, Jiang Z, Yu G, Li Y, Quinlan EM, and Nakazawa K (2010) Postnatal NMDA receptor ablation in corticolimbic interneurons confers schizophrenia-like phenotypes. Nat Neurosci 13:76-83.

Belsky J, Jonassaint C, Pluess M, Stanton M, Brummett B, and Williams R (2009) Vulnerability genes or plasticity genes?. Mol Psychiatry 14:746-754

Belujon P and Grace AA (2014) Restoring mood balance in depression: ketamine reverses deficit in dopamine-dependent synaptic plasticity. Biol Psychiatry 76:927-936.

Belujon P and Grace AA (2017) Dopamine system dysregulation in major depressive disorders. Int $J$ Neuropsychopharmacol 20:1036-1046.

Belujon P, Jakobowski NL, Dollish HK, and Grace AA (2016) Withdrawal from acute amphetamine induces an amygdala-driven attenuation of dopamine neuron activity: reversal by ketamine. Neuropsychopharmacology 41:619-627.

Bengel D, Murphy DL, Andrews AM, Wichems CH, Feltner D, Heils A, Mössner R, Westphal H, and Lesch KP (1998) Altered brain serotonin homeostasis and locomotor insensitivity to 3, 4-methylenedioxymethamphetamine ("Ecstasy") in serotonin transporter-deficient mice. Mol Pharmacol 53:649-655.

Bennett MR (2010) Synapse regression in depression: the role of 5-HT receptors in modulating NMDA receptor function and synaptic plasticity. Aust $N Z$ J Psychiatry 44:301-308.

Benneyworth MA, Xiang Z, Smith RL, Garcia EE, Conn PJ, and Sanders-Bush E (2007) A selective positive allosteric modulator of metabotropic glutamate receptor subtype 2 blocks a hallucinogenic drug model of psychosis. Mol Pharmacol 72: $477-484$

Berge OG, Chacho D, and Hole K (1983) Inhibitory effect of 5-methoxy-N,N-dimethyltryptamine on the synaptosomal uptake of 5-hydroxytryptamine. Eur $J$ Pharmacol 90:293-296.

Berman RM, Cappiello A, Anand A, Oren DA, Heninger GR, Charney DS, and Krystal $\mathrm{JH}$ (2000) Antidepressant effects of ketamine in depressed patients. Biol Psychiatry 47:351-354.

Berrens Z, Lammers J, and White C (2010) Rhabdomyolysis after LSD ingestion. Psychosomatics 51:356-356.e3.

Berridge CW and Waterhouse BD (2003) The locus coeruleus-noradrenergic system modulation of behavioral state and state-dependent cognitive processes. Brain Res Brain Res Rev 42:33-84.

Bershad AK, Preller KH, Lee R, Keedy S, Wren-Jarvis J, Bremmer MP, and de Wit H (2020) Preliminary report on the effects of a low dose of LSD on resting-state amygdala functional connectivity. Biol Psychiatry Cogn Neurosci Neuroimaging 5 461-467.

Bershad AK, Schepers ST, Bremmer MP, Lee R, and de Wit H (2019) Acute subjective and behavioral effects of Microdoses of lysergic acid diethylamide in healthy human volunteers. Biol Psychiatry 86:792-800. 
Bershad AK, Weafer JJ, Kirkpatrick MG, Wardle MC, Miller MA, and de Wit H (2016) Oxytocin receptor gene variation predicts subjective responses to MDMA. Soc Neurosci 11:592-599.

Bertron JL, Seto M, and Lindsley CW (2018) DARK classics in chemical neuroscience: phencyclidine (PCP). ACS Chem Neurosci 9:2459-2474.

Beucke JC, Sepulcre J, Eldaief MC, Sebold M, Kathmann N, and Kaufmann C (2014) Default mode network subsystem alterations in obsessive-compulsive disorder. $B r$ J Psychiatry 205:376-382.

Bevan RK, Rose MA, and Duggan KA (1997) Evidence for direct interaction of ketamine with alpha 1- and beta 2-adrenoceptors. Clin Exp Pharmacol Physiol 24: 923-926.

Bexis S and Docherty JR (2005) Role of alpha2A-adrenoceptors in the effects of MDMA on body temperature in the mouse. Br J Pharmacol 146:1-6.

Bexis S and Docherty JR (2009) Role of alpha 1- and beta 3-adrenoceptors in the modulation by SR59230A of the effects of MDMA on body temperature in the mouse. Br J Pharmacol 158.259-266.

Biezonski DK, Piper BJ, Shinday NM, Kim PJ, Ali SF, and Meyer JS (2013) Effects of a short-course MDMA binge on dopamine transporter binding and on levels of dopamine and its metabolites in adult male rats. Eur J Pharmacol 701:176-180.

Bílková J, Radil-Weiss T, and Bohdanecký Z (1971a) The influence of low LSD dose administration during sleep in rats. Psychopharmacology (Berl) 20:395-399.

Bílková J, Radil-Weiss T, and Bohdanecky Z (1971b) The influence of LSD on sleep cycles in rats. Act Nerv Super (Praha) 13:100-101.

Bird MK, Kirchhoff J, Djouma E, and Lawrence AJ (2008) Metabotropic glutamate 5 receptors regulate sensitivity to ethanol in mice. Int $J$ Neuropsychopharmacol 11: $765-774$

Blaho K, Merigian K, Winbery S, Geraci SA, and Smartt C (1997) Clinical pharmacology of lysergic acid diethylamide: case reports and review of the treatment of intoxication. Am $J$ Ther 4:211-221.

Blandino-Rosano M, Barbaresso R, Jimenez-Palomares M, Bozadjieva N, Werneckde-Castro JP, Hatanaka M, Mirmira RG, Sonenberg N, Liu M, Rüegg MA, et al (2017) Loss of mTORC1 signalling impairs $\beta$-cell homeostasis and insulin processing. Nat Commun 8:16014.

Blesa J and Przedborski S (2014) Parkinson's disease: animal models and dopaminergic cell vulnerability. Front Neuroanat 8:155.

Blier P and de Montigny C (1994) Current advances and trends in the treatment of depression. Trends Pharmacol Sci 15:220-226.

Blier P, Lista A, and De Montigny C (1993) Differential properties of pre- and postsynaptic 5-hydroxytryptamine1A receptors in the dorsal raphe and hippocampus: I. Effect of spiperone. J Pharmacol Exp Ther 265:7-15.

Blough BE, Landavazo A, Decker AM, Partilla JS, Baumann MH, and Rothman RB (2014) Interaction of psychoactive tryptamines with biogenic amine transporter and serotonin receptor subtypes. Psychopharmacology (Berl) 231:4135-4144.

Bogenschutz MP, Forcehimes AA, Pommy JA, Wilcox CE, Barbosa PC, and Strassman RJ (2015) Psilocybin-assisted treatment for alcohol dependence: a proof-of-concept study. J Psychopharmacol 29:289-299.

Bolton WB (1962) 'Schizophrenia' produced by LSD-25. Nurs Times 58:22-24.

Bonson KR, Buckholtz JW, and Murphy DL (1996) Chronic administration of serotonergic antidepressants attenuates the subjective effects of LSD in humans. Neuropsychopharmacology 14:425-436.

Borowiak KS, Ciechanowski K, and Waloszczyk P (1998) Psilocybin mushroom (Psilocybe semilanceata) intoxication with myocardial infarction. $J$ Toxicol Clin Toxicol 36:47-49.

Borowski TB and Kokkinidis L (1998) The effects of cocaine, amphetamine, and the dopamine D1 receptor agonist SKF 38393 on fear extinction as measured with potentiated startle: implications for psychomotor stimulant psychosis. Behav Neurosci 112:952-965.

Borroto-Escuela DO, Carlsson J, Ambrogini P, Narváez M, Wydra K, Tarakanov AO, Li X, Millón C, Ferraro L, Cuppini R, et al. (2017a) Understanding the role of GPCR heteroreceptor complexes in modulating the brain networks in health and disease. Front Cell Neurosci 11:37.

Borroto-Escuela DO, Li X, Tarakanov AO, Savelli D, Narváez M, Shumilov K, Andrade-Talavera Y, Jimenez-Beristain A, Pomierny B, Díaz-Cabiale Z, et al. (2017b) Existence of brain 5-HT1A-5-HT2A isoreceptor complexes with antagonistic allosteric receptor-receptor interactions regulating 5-HT1A receptor recognition. ACS Omega 2:4779-4789.

Borroto-Escuela DO, Romero-Fernandez W, Narvaez M, Oflijan J, Agnati LF, and Fuxe K (2014) Hallucinogenic 5-HT2AR agonists LSD and DOI enhance dopamine D2R protomer recognition and signaling of D2-5-HT2A heteroreceptor complexes. Biochem Biophys Res Commun 443:278-284.

Bosch-Bouju C, Hyland BI, and Parr-Brownlie LC (2013) Motor thalamus integration of cortical, cerebellar and basal ganglia information: implications for normal and parkinsonian conditions. Front Comput Neurosci 7:163

Bouso JC, Dos Santos RG, Alcázar-Córcoles MA, and Hallak JEC (2018) Serotonergic psychedelics and personality: a systematic review of contemporary research. Neurosci Biobehav Rev 87:118-132

Bouso JC, Fábregas JM, Antonijoan RM, Rodríguez-Fornells A, and Riba J (2013) Acute effects of ayahuasca on neuropsychological performance: differences in executive function between experienced and occasional users. Psychopharmacology (Berl) 230:415-424.

Bouso JC, González D, Fondevila S, Cutchet M, Fernández X, Ribeiro Barbosa PC, Alcázar-Córcoles MA, Araújo WS, Barbanoj MJ, Fábregas JM, et al. (2012) Personality, psychopathology, life attitudes and neuropsychological performance among ritual users of Ayahuasca: a longitudinal study. PLoS One 7:e42421.

Bouso JC, Palhano-Fontes F, Rodríguez-Fornells A, Ribeiro S, Sanches R, Crippa JA, Hallak JE, de Araujo DB, and Riba J (2015) Long-term use of psychedelic drugs is associated with differences in brain structure and personality in humans. Eur Neuropsychopharmacol 25:483-492.

Boxler MI, Streun GL, Liechti ME, Schmid Y, Kraemer T, and Steuer AE (2018) Human metabolome changes after a single dose of 3,4-Methylenedioxymethamphetamine
(MDMA) with special focus on steroid metabolism and inflammation processes. $J$ Proteome Res 17:2900-2907.

Boyer EW and Shannon M (2005) The serotonin syndrome. N Engl J Med 352 1112-1120.

Boyle NT and Connor TJ (2010) Methylenedioxymethamphetamine ('Ecstasy')-induced immunosuppression: a cause for concern?. Br J Pharmacol 161:17-32.

Brammer MK, Gilmore DL, and Matsumoto RR (2006) Interactions between 3,4methylenedioxymethamphetamine and sigma1 receptors. Eur $J$ Pharmacol 553: 141-145.

Brandt SD, Kavanagh PV, Westphal F, Elliott SP, Wallach J, Stratford A, Nichols DE, and Halberstadt AL (2017) Return of the lysergamides. Part III: analytical characterization of $\mathrm{N}^{6}$-ethyl-6-norlysergic acid diethylamide (ETH-LAD) and 1 propionyl ETH-LAD (1P-ETH-LAD). Drug Test Anal 9:1641-1649.

Breier A, Adler CM, Weisenfeld N, Su TP, Elman I, Picken L, Malhotra AK, and Pickar D (1998) Effects of NMDA antagonism on striatal dopamine release in healthy subjects: application of a novel PET approach. Synapse 29:142-147.

Bremner JD, Narayan M, Anderson ER, Staib LH, Miller HL, and Charney DS (2000) Hippocampal volume reduction in major depression. Am J Psychiatry 157:115-118. Brown EC, Clark DL, Hassel S, MacQueen G, and Ramasubbu R (2017a) Thalamocortical connectivity in major depressive disorder. J Affect Disord 217:125-131.

Brown PJ and Wolfe J (1994) Substance abuse and post-traumatic stress disorder comorbidity. Drug Alcohol Depend 35:51-59.

Brown RT, Nicholas CR, Cozzi NV, Gassman MC, Cooper KM, Muller D, Thomas CD, Hetzel SJ, Henriquez KM, Ribaudo AS, et al. (2017b) Pharmacokinetics of escalating doses of oral psilocybin in healthy adults. Clin Pharmacokinet 56: 1543-1554

Brown T, Shao W, Ayub S, Chong D, and Cornelius C (2017c) A physician's attempt to self-medicate bipolar depression with N,N-dimethyltryptamine (DMT). J Psychoactive Drugs 49:294-296.

Buchborn T, Schröder H, Höllt V, and Grecksch G (2014) Repeated lysergic acid diethylamide in an animal model of depression: normalisation of learning behaviour and hippocampal serotonin 5-HT2 signalling. J Psychopharmacol 28:545-552

Buckholtz NS, Zhou DF, Freedman DX, and Potter WZ (1990) Lysergic acid diethylamide (LSD) administration selectively downregulates serotonin2 receptors in rat brain. Neuropsychopharmacology 3:137-148.

Buckner RL, Andrews-Hanna JR, and Schacter DL (2008) The brain's default network: anatomy, function, and relevance to disease. Ann N Y Acad Sci 1124:1-38.

Budd RD and Lindstrom DM (1982) Characteristics of victims of PCP-related death in Los Angeles County. J Toxicol Clin Toxicol 19:997-1004.

Bunzow JR, Sonders MS, Arttamangkul S, Harrison LM, Zhang G, Quigley DI, Darland T, Suchland KL, Pasumamula S, Kennedy JL, et al. (2001) Amphetamine, 3,4-methylenedioxymethamphetamine, lysergic acid diethylamide, and metabolites of the catecholamine neurotransmitters are agonists of a rat trace amine receptor. Mol Pharmacol 60:1181-1188.

Caldeira MV, Melo CV, Pereira DB, Carvalho R, Correia SS, Backos DS, Carvalho AL, Esteban JA, and Duarte CB (2007) Brain-derived neurotrophic factor regulate the expression and synaptic delivery of alpha-amino-3-hydroxy-5-methyl-4-isoxazole propionic acid receptor subunits in hippocampal neurons. J Biol Chem $\mathbf{2 8 2}$ 12619-12628.

Callaway JC, Airaksinen MM, McKenna DJ, Brito GS, and Grob CS (1994) Platelet serotonin uptake sites increased in drinkers of ayahuasca. Psychopharmacology (Berl) 116:385-387.

Callaway JC, McKenna DJ, Grob CS, Brito GS, Raymon LP, Poland RE, Andrade EN, Andrade EO, and Mash DC (1999) Pharmacokinetics of Hoasca alkaloids in healthy humans. J Ethnopharmacol 65:243-256.

Cameron LP, Benson CJ, DeFelice BC, Fiehn O, and Olson DE (2019) Chronic, intermittent microdoses of the psychedelic $N, N$-dimethyltryptamine (DMT) produce positive effects on mood and anxiety in rodents. ACS Chem Neurosci 10:3261-3270. Can A, Zanos P, Moaddel R, Kang HJ, Dossou KS, Wainer IW, Cheer JF, Frost DO, Huang XP, and Gould TD (2016) Effects of ketamine and ketamine metabolites on evoked striatal dopamine release, dopamine receptors, and monoamine transporters. J Pharmacol Exp Ther 359:159-170.

Canal CE and Murnane KS (2017) The serotonin $5-\mathrm{HT}_{2 \mathrm{C}}$ receptor and the non addictive nature of classic hallucinogens. J Psychopharmacol 31:127-143.

Carayon P, Bouaboula M, Loubet JF, Bourrie B, Petitpretre G, Le Fur G, and Casellas P (1995) The sigma ligand SR 31747 prevents the development of acute graft-versus-host disease in mice by blocking IFN-gamma and GM-CSF mRNA expression. Int $J$ Immunopharmacol 17:753-761.

Carbonaro TM, Eshleman AJ, Forster MJ, Cheng K, Rice KC, and Gatch MB (2015) The role of 5-HT2A, 5-HT 2C and mGlu2 receptors in the behavioral effects of tryptamine hallucinogens $\mathrm{N}, \mathrm{N}$-dimethyltryptamine and N,N-diisopropyltryptamine in rats and mice. Psychopharmacology (Berl) 232:275-284.

Cardenas VA, Samuelson K, Lenoci M, Studholme C, Neylan TC, Marmar CR, Schuff $\mathrm{N}$, and Weiner MW (2011) Changes in brain anatomy during the course of posttraumatic stress disorder. Psychiatry Res 193:93-100.

Carhart-Harris RL, Bolstridge M, Day CMJ, Rucker J, Watts R, Erritzoe DE, Kaelen M, Giribaldi B, Bloomfield M, Pilling S, et al. (2018a) Psilocybin with psychological support for treatment-resistant depression: six-month follow-up. Psychopharmacology (Berl) 235:399-408.

Carhart-Harris RL, Bolstridge M, Rucker J, Day CM, Erritzoe D, Kaelen M, Bloomfield M, Rickard JA, Forbes B, Feilding A, et al. (2016a) Psilocybin with psychological support for treatment-resistant depression: an open-label feasibility study. Lancet Psychiatry 3:619-627.

Carhart-Harris RL, Erritzoe D, Williams T, Stone JM, Reed LJ, Colasanti A, Tyacke RJ, Leech R, Malizia AL, Murphy K, et al. (2012) Neural correlates of the psychedelic state as determined by fMRI studies with psilocybin. Proc Natl Acad Sci USA 109:2138-2143.

Carhart-Harris RL, Kaelen M, Bolstridge M, Williams TM, Williams LT, Underwood R, Feilding A, and Nutt DJ (2016b) The paradoxical psychological effects of lysergic acid diethylamide (LSD). Psychol Med 46:1379-1390. 
Carhart-Harris RL, Kaelen M, Whalley MG, Bolstridge M, Feilding A, and Nutt DJ (2015) LSD enhances suggestibility in healthy volunteers. Psychopharmacology (Berl) 232:785-794.

Carhart-Harris RL, Muthukumaraswamy S, Roseman L, Kaelen M, Droog W, Murphy K, Tagliazucchi E, Schenberg EE, Nest T, Orban C, et al. (2016c) Neural correlates of the LSD experience revealed by multimodal neuroimaging. Proc Natl Acad Sci USA 113:4853-4858.

Carhart-Harris RL, Leech R, Erritzoe D, Williams TM, Stone JM, Evans J, Sharp DJ, Feilding A, Wise RG, and Nutt DJ (2013) Functional connectivity measures after psilocybin inform a novel hypothesis of early psychosis. Schizophr Bull 39: 1343-1351.

Carhart-Harris RL, Roseman L, Bolstridge M, Demetriou L, Pannekoek JN, Wall MB, Tanner M, Kaelen M, McGonigle J, Murphy K, et al. (2017) Psilocybin for treatment-resistant depression: fMRI-measured brain mechanisms. Sci Rep 7 13187.

Carhart-Harris RL, Roseman L, Haijen E, Erritzoe D, Watts R, Branchi I, and Kaelen M (2018b) Psychedelics and the essential importance of context. J Psychopharmacol 32:725-731.

Carlson EB, Waelde LC, Palmieri PA, Macia KS, Smith SR, and McDade-Montez E (2018) Development and validation of the dissociative symptoms scale. Assessment 25:84-98.

Carlsson A and Carlsson ML (2008) Adaptive properties and heterogeneity of dopamine $\mathrm{D}(2)$ receptors - pharmacological implications. Brain Res Brain Res Rev $\mathbf{5 8}$ 374-378.

Carspecken CW, Borisovskaya A, Lan ST, Heller K, Buchholz J, Ruskin D, and Rozet I (2018) Ketamine anesthesia does not improve depression scores in electroconvulsive therapy: a randomized clinical trial. J Neurosurg Anesthesiol 30:305-313.

Carter OL, Burr DC, Pettigrew JD, Wallis GM, Hasler F, and Vollenweider FX (2005) Using psilocybin to investigate the relationship between attention, working memory, and the serotonin $1 \mathrm{~A}$ and 2A receptors. J Cogn Neurosci 17:1497-1508.

Carvalho LA and Pariante CM (2008) In vitro modulation of the glucocorticoid receptor by antidepressants. Stress 11:411-424

Caspi A, Hariri AR, Holmes A, Uher R, and Moffitt TE (2010) Genetic sensitivity to the environment: the case of the serotonin transporter gene and its implications for studying complex diseases and traits. Am J Psychiatry 167:509-527.

Caspi A, Sugden K, Moffitt TE, Taylor A, Craig IW, Harrington H, McClay J, Mill J, Martin J, Braithwaite A, et al. (2003) Influence of life stress on depression: moderation by a polymorphism in the 5-HTT gene. Science 301:386-389.

Castagnoli N Jr and Mantle PG (1966) Occurrence of D-lysergic acid and 6-methylergol-8-ene-8-carboxylic acid in cultures of Claviceps purpurea. Nature 211: $859-860$

Cathala A, Devroye C, Drutel G, Revest JM, Artigas F, and Spampinato U (2019)

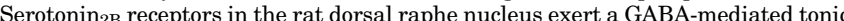
inhibitory control on serotonin neurons. Exp Neurol 311:57-66.

Catlow BJ, Song S, Paredes DA, Kirstein CL, and Sanchez-Ramos J (2013) Effects of psilocybin on hippocampal neurogenesis and extinction of trace fear conditioning. Exp Brain Res 228:481-491.

Celada P, Puig M, Amargós-Bosch M, Adell A, and Artigas F (2004) The therapeutic role of 5-HT1A and 5-HT2A receptors in depression. $J$ Psychiatry Neurosci 29: $252-265$.

Chadeayne AR, Pham DN, Reid BG, Golen JA, and Manke DR (2020) Active metabolite of aeruginascin (4-hydroxy- N, N, N-trimethyltryptamine): synthesis, structure, and serotonergic binding affinity. ACS Omega 5:16940-16943.

Char D, Drover DR, Motonaga KS, Gupta S, Miyake CY, Dubin AM, and Hammer GB (2013) The effects of ketamine on dexmedetomidine-induced electrophysiologic changes in children. Paediatr Anaesth 23:898-905.

Chaudhry IB, Hallak J, Husain N, Minhas F, Stirling J, Richardson P, Dursun S, Dunn G, and Deakin B (2012) Minocycline benefits negative symptoms in early schizophrenia: a randomised double-blind placebo-controlled clinical trial in patients on standard treatment. J Psychopharmacol 26:1185-1193.

Che S, Johnson M, Hanson GR, and Gibb JW (1995) Body temperature effect on methylenedioxymethamphetamine-induced acute decrease in tryptophan hydroxylase activity. Eur J Pharmacol 293:447-453.

Cheek FE and Holstein CM (1971) Lysergic acid diethylamide tartrate (LSD-25) dosage levels, group differences and social interaction. J Nerv Ment Dis 153: 133-147.

Cheung SG, Goldenthal AR, Uhlemann AC, Mann JJ, Miller JM, and Sublette ME (2019) Systematic review of gut microbiota and major depression. Front Psychiatry 10:34.

Chia Z, Augustine GJ, and Silberberg G (2020) Synaptic connectivity between the cortex and claustrum is organized into functional modules. Curr Biol 30: 2777-2790.e4.

Choi M, Lee SH, Park MH, Kim YS, and Son H (2017) Ketamine induces brainderived neurotrophic factor expression via phosphorylation of histone deacetylase 5 in rats. Biochem Biophys Res Commun 489:420-425.

Cholden LS, Kurland A, and Savage C (1955) Clinical reactions and tolerance to LSD in chronic schizophrenia. J Nerv Ment Dis 122:211-221.

Choudary PV, Molnar M, Evans SJ, Tomita H, Li JZ, Vawter MP, Myers RM, Bunney WE Jr, Akil H, Watson SJ, et al. (2005) Altered cortical glutamatergic and GABAergic signal transmission with glial involvement in depression. Proc Natl Acad Sci USA 102:15653-15658.

Chourbaji S, Vogt MA, Fumagalli F, Sohr R, Frasca A, Brandwein C, Hörtnagl H, Riva MA, Sprengel R, and Gass P (2008) AMPA receptor subunit 1 (GluR-A) knockout mice model the glutamate hypothesis of depression. FASEB $J \mathbf{2 2}$ : 3129-3134.

Clark PJ, Kohman RA, Miller DS, Bhattacharya TK, Haferkamp EH, and Rhodes JS (2010) Adult hippocampal neurogenesis and c-Fos induction during escalation of voluntary wheel running in C57BL/6J mice. Behav Brain Res 213:246-252.

Cochran DM, Fallon D, Hill M, and Frazier JA (2013) The role of oxytocin in psychiatric disorders: a review of biological and therapeutic research findings. Harv Rev Psychiatry 21:219-247.
Cogen FC, Rigg G, Simmons JL, and Domino EF (1978) Phencyclidine-associated acute rhabdomyolysis. Ann Intern Med 88:210-212.

Cohen S and Eisner BG (1959) Use of lysergic acid diethylamide in a psychotherapeutic setting. AMA Arch Neurol Psychiatry 81:615-619.

Colaço CS, Alves SS, Nolli LM, Pinheiro WO, de Oliveira DGR, Santos BWL, PicTaylor A, Mortari MR, and Caldas ED (2020) Toxicity of ayahuasca after 28 days daily exposure and effects on monoamines and brain-derived neurotrophic factor (BDNF) in brain of Wistar rats. Metab Brain Dis 35:739-751.

Colado MI, O'Shea E, Esteban B, Granados R, and Green AR (1999) In vivo evidence against clomethiazole being neuroprotective against MDMA ('ecstasy')-induced degeneration of rat brain 5-HT nerve terminals by a free radical scavenging mechanism. Neuropharmacology 38:307-314

Cole JC and Sumnall HR (2003) Altered states: the clinical effects of Ecstasy. Pharmacol Ther 98:35-58.

Collins SA, Gudelsky GA, and Yamamoto BK (2015) MDMA-induced loss of parvalbumin interneurons within the dentate gyrus is mediated by 5HT2A and NMDA receptors. Eur J Pharmacol 761:95-100.

Comai S, Tau M, Pavlovic Z, and Gobbi G (2012) The psychopharmacology of aggressive behavior: a translational approach: part 2: clinical studies using atypical antipsychotics, anticonvulsants, and lithium. J Clin Psychopharmacol 32:237-260.

Conibear AE and Kelly E (2019) A biased view of $\mu$-opioid receptors?. Mol Pharmacol 96:542-549.

Connolly HM, Crary JL, McGoon MD, Hensrud DD, Edwards BS, Edwards WD, and Schaff HV (1997) Valvular heart disease associated with fenfluraminephentermine. $N$ Engl J Med 337:581-588.

Connor TJ, Kelly JP, and Leonard BE (2000) An assessment of the acute effects of the serotonin releasers methylenedioxymethamphetamine, methylenedioxyamphetamine and fenfluramine on immunity in rats. Immunopharmacology 46:223-235.

Cooper JM, Newby DA, Whyte IM, Carter G, Jones AL, and Isbister GK (2014) Serotonin toxicity from antidepressant overdose and its association with the T102C polymorphism of the 5-HT2A receptor. Pharmacogenomics $J$ 14:390-394.

Cornwell BR, Salvadore G, Furey M, Marquardt CA, Brutsche NE, Grillon C, and Zarate CA Jr (2012) Synaptic potentiation is critical for rapid antidepressant response to ketamine in treatment-resistant major depression. Biol Psychiatry $\mathbf{7 2}$ $555-561$.

Costa B, Pini S, Gabelloni P, Abelli M, Lari L, Cardini A, Muti M, Gesi C, Landi S, Galderisi S, et al. (2009) Oxytocin receptor polymorphisms and adult attachment style in patients with depression. Psychoneuroendocrinology 34:1506-1514.

Costa-Mattioli M and Monteggia LM (2013) mTOR complexes in neurodevelopmental and neuropsychiatric disorders. Nat Neurosci 16:1537-1543.

Coutinho JF, Fernandesl SV, Soares JM, Maia L, Gonçalves OF, and Sampaio A (2016) Default mode network dissociation in depressive and anxiety states. Brain Imaging Behav 10:147-157.

Coyle JT (2012) NMDA receptor and schizophrenia: a brief history. Schizophr Bull 38:920-926.

Cozzi NV, Gopalakrishnan A, Anderson LL, Feih JT, Shulgin AT, Daley PF, and Ruoho AE (2009) Dimethyltryptamine and other hallucinogenic tryptamines exhibit substrate behavior at the serotonin uptake transporter and the vesicle monoamine transporter. J Neural Transm (Vienna) 116:1591-1599.

Cramer SC, Sur M, Dobkin BH, O’Brien C, Sanger TD, Trojanowski JQ, Rumsey JM Hicks R, Cameron J, Chen D, et al. (2011) Harnessing neuroplasticity for clinical applications. Brain 134:1591-1609.

Crick FC and Koch C (2005) What is the function of the claustrum?. Philos Trans $R$ Soc Lond B Biol Sci 360:1271-1279.

Crider JY, Williams GW, Drace CD, Katoli P, Senchyna M, and Sharif NA (2003) Pharmacological characterization of a serotonin receptor (5-HT7) stimulating cAMP production in human corneal epithelial cells. Invest Ophthalmol Vis Sci $\mathbf{4 4}$ 4837-4844.

Crisp T, Perrotti JM, Smith DL, Stafinsky JL, and Smith DJ (1991) The local monoaminergic dependency of spinal ketamine. Eur J Pharmacol 194:167-172.

Cristea IA and Naudet F (2019) US Food and Drug Administration approval of esketamine and brexanolone. Lancet Psychiatry 6:975-977.

Cross-Disorder Group of the Psychiatric Genomics Consortium (2013) Identification of risk loci with shared effects on five major psychiatric disorders: a genome-wide analysis. Lancet 381:1371-1379.

Cullen KR, Amatya P, Roback MG, Albott CS, Westlund Schreiner M, Ren Y, Eberly LE, Carstedt P, Samikoglu A, Gunlicks-Stoessel M, et al. (2018) Intravenous ketamine for adolescents with treatment-resistant depression: an open-label study. J Child Adolesc Psychopharmacol 28:437-444.

Cumming-Hood PA, Strahlendorf HK, and Strahlendorf JC (1993) Effects of serotonin and the 5 -HT2/1C receptor agonist DOI on neurons of the cerebellar dentate/ interpositus nuclei: possible involvement of a GABAergic interneuron. Eur $J$ Pharmacol 236:457-465

Curfman GD (1997) Diet pills redux. N Engl J Med 337:629-630.

Cusin C, Ionescu DF, Pavone KJ, Akeju O, Cassano P, Taylor N, Eikermann M Durham K, Swee MB, Chang T, et al. (2017) Ketamine augmentation for outpatients with treatment-resistant depression: preliminary evidence for two-step intravenous dose escalation. Aust $N Z$ J Psychiatry 51:55-64

Dakic V, Minardi Nascimento J, Costa Sartore R, Maciel RM, de Araujo DB, Ribeiro S, Martins-de-Souza D, and Rehen SK (2017) Short term changes in the proteome of human cerebral organoids induced by 5-MeO-DMT. Sci Rep 7:12863.

Dakwar E, Hart CL, Levin FR, Nunes EV, and Foltin RW (2017) Cocaine selfadministration disrupted by the N-methyl-D-aspartate receptor antagonist ketamine: a randomized, crossover trial. Mol Psychiatry 22:76-81.

Dakwar E, Levin F, Foltin RW, Nunes EV, and Hart CL (2014) The effects of subanesthetic ketamine infusions on motivation to quit and cue-induced craving in cocaine-dependent research volunteers. Biol Psychiatry 76:40-46.

Dakwar E, Levin F, Hart CL, Basaraba C, Choi J, Pavlicova M, and Nunes EV (2020) A single ketamine infusion combined with motivational enhancement therapy for alcohol use disorder: a randomized midazolam-controlled pilot trial. Am J Psychiatry 177:125-133. 
Dakwar E, Nunes EV, Hart CL, Foltin RW, Mathew SJ, Carpenter KM, Choi CJJ, Basaraba CN, Pavlicova M, and Levin FR (2019) A single ketamine infusion combined with mindfulness-based behavioral modification to treat cocaine dependence: a randomized clinical trial. Am J Psychiatry 176:923-930.

Danforth AL, Grob CS, Struble C, Feduccia AA, Walker N, Jerome L, Yazar-Klosinsk B, and Emerson A (2018) Reduction in social anxiety after MDMA-assisted psychotherapy with autistic adults: a randomized, double-blind, placebo-controlled pilot study. Psychopharmacology (Berl) 235:3137-3148.

Danforth AL, Struble CM, Yazar-Klosinski B, and Grob CS (2016) MDMA-assisted therapy: a new treatment model for social anxiety in autistic adults. Prog Neuropsychopharmacol Biol Psychiatry 64:237-249.

Dantzer R, O'Connor JC, Freund GG, Johnson RW, and Kelley KW (2008) From inflammation to sickness and depression: when the immune system subjugates the brain. Nat Rev Neurosci 9:46-56.

da Silva FS, Silva EAS, Sousa GM Jr, Maia-de-Oliveira JP, Soares-Rachetti VP, de Araujo DB, Sousa MBC, Lobão-Soares B, Hallak J, and Galvão-Coelho NL (2019) Acute effects of ayahuasca in a juvenile non-human primate model of depression. Br J Psychiatry 41:280-288.

Da Silveira DX, Grob CS, de Rios MD, Lopez E, Alonso LK, Tacla C, and Doering Silveira E (2005) Ayahuasca in adolescence: a preliminary psychiatric assessment. J Psychoactive Drugs 37:129-133.

Davies O, Batajoo-Shrestha B, Sosa-Popoteur J, and Olibrice M (2014) Full recovery after severe serotonin syndrome, severe rhabdomyolysis, multi-organ failure and disseminated intravascular coagulopathy from MDMA. Heart Lung 43:117-119.

Davis AK, Barrett FS, and Griffiths RR (2020) Psychological flexibility mediates the relations between acute psychedelic effects and subjective decreases in depression and anxiety. J Contextual Behav Sci 15:39-45.

Davis AK, Barrett FS, May DG, Cosimano MP, Sepeda ND, Johnson MW, Finan PH, and Griffiths RR (2020) Effects of psilocybin-sssisted therapy on major depressive disorder. AMA Psychiatry, doi: 10.1001/jamapsychiatry.2020.3285.

Davis AK, So S, Lancelotta R, Barsuglia JP, and Griffiths RR (2019) 5-methoxy-N,Ndimethyltryptamine (5-MeO-DMT) used in a naturalistic group setting is associated with unintended improvements in depression and anxiety. Am J Drug Alcohol Abuse 45:161-169.

Davis KL, Kahn RS, Ko G, and Davidson M (1991) Dopamine in schizophrenia: a review and reconceptualization. Am J Psychiatry 148:1474-1486.

Dawson N, Morris BJ, and Pratt JA (2013) Subanaesthetic ketamine treatment alters prefrontal cortex connectivity with thalamus and ascending subcortical systems. Schizophr Bull 39:366-377.

de Almeida RN, Galvão ACM, da Silva FS, Silva EADS, Palhano-Fontes F, Maia-deOliveira JP, de Araújo LB, Lobão-Soares B, and Galvão-Coelho NL (2019) Modulation of serum brain-derived neurotrophic factor by a single dose of ayahuasca: observation from a randomized controlled trial. Front Psychol 10:1234.

Dean BV, Stellpflug SJ, Burnett AM, and Engebretsen KM (2013) 2C or not 2C: phenethylamine designer drug review. J Med Toxicol 9:172-178.

Dean JG, Liu T, Huff S, Sheler B, Barker SA, Strassman RJ, Wang MM, and Borjigin $\mathrm{J}$ (2019) Biosynthesis and extracellular concentrations of N,N-dimethyltryptamine (DMT) in mammalian brain. Sci Rep 9:9333.

Dean OM, Kanchanatawan B, Ashton M, Mohebbi M, Ng CH, Maes M, Berk L, Sughondhabirom A, Tangwongchai S, Singh AB, et al. (2017) Adjunctive minocycline treatment for major depressive disorder: a proof of concept trial. Aust $N Z$ J Psychiatry 51:829-840.

De Blasi A (1990) Beta-adrenergic receptors: structure, function and regulation. Drugs Exp Clin Res 16:107-112.

de Brabander JM, Kramers RJ, and Uylings HB (1998) Layer-specific dendritic regression of pyramidal cells with ageing in the human prefrontal cortex. Eur J Neurosci 10:1261-1269.

de Castro-Neto EF, da Cunha RH, da Silveira DX, Yonamine M, Gouveia TL, Cavalheiro EA, Amado D, and Naffah-Mazzacoratti MdaG (2013) Changes in aminoacidergic and monoaminergic neurotransmission in the hippocampus and amygdala of rats after ayahuasca ingestion. World $J$ Biol Chem 4:141-147.

De Gregorio D, Comai S, Posa L, and Gobbi G (2016a) d-Lysergic Acid Diethylamide (LSD) as a model of psychosis: mechanism of action and pharmacology. Int J Mol Sci 17:1953.

De Gregorio D, Enns JP, Nuñez NA, Posa L, and Gobbi G (2018) d-Lysergic acid diethylamide, psilocybin, and other classic hallucinogens: mechanism of action and potential therapeutic applications in mood disorders. Prog Brain Res 242:69-96.

De Gregorio D, Posa L, Ochoa-Sanchez R, McLaughlin R, Maione S, Comai S, and Gobbi G (2016b) The hallucinogen d-lysergic diethylamide (LSD) decreases dopamine firing activity through $5-\mathrm{HT}_{1 \mathrm{~A}}, \mathrm{D}_{2}$ and $\mathrm{TAAR}_{1}$ receptors. Pharmacol Res 113:81-91.

Deliganis AV, Pierce PA, and Peroutka SJ (1991) Differential interactions of dimethyltryptamine (DMT) with 5-HT1A and 5-HT2 receptors. Biochem Pharmacol 41:1739-1744.

Delille HK, Mezler M, and Marek GJ (2013) The two faces of the pharmacological interaction of mGlu2 and $5-\mathrm{HT}_{2} \mathrm{~A}$ - relevance of receptor heterocomplexes and interaction through functional brain pathways. Neuropharmacology 70:296-305.

Dell'Osso L, Carmassi C, Del Debbio A, Catena Dell'Osso M, Bianchi C, da Pozzo E, Origlia N, Domenici L, Massimetti G, Marazziti D, et al. (2009) Brain-derived neurotrophic factor plasma levels in patients suffering from post-traumatic stress disorder. Prog Neuropsychopharmacol Biol Psychiatry 33:899-902.

Demmerle J, Koch AJ, and Holaska JM (2012) The nuclear envelope protein emerin binds directly to histone deacetylase 3 (HDAC3) and activates HDAC3 activity. $J$ Biol Chem 287:22080-22088.

Dengler HJ, Spiegel HE, and Titus EO (1961) Effects of drugs on uptake of isotopic norepinephrine by cat tissues. Nature 191:816-817.

Denson R and Sydiaha D (1970) A controlled study of LSD treatment in alcoholism and neurosis. $B r J$ Psychiatry 116:443-445

de Oliveira CDR, Moreira CQ, Spinosa HdS, and Yonamine M (2011) Neurobehavioral, reflexological and physical development of Wistar rat offspring exposed to ayahuasca during pregnancy and lactation. Rev Bras Farmacogn 21:1065-1076.
Depoortere H and Loew DM (1971) Alterations in sleep-wakefulness cycle in rats following treatment with (+)-lysergic acid diethylamide (LSD-25). Br J Pharmacol 41:402P-403P

DeWire SM, Yamashita DS, Rominger DH, Liu G, Cowan CL, Graczyk TM, Chen X-T, Pitis PM, Gotchev D, Yuan C, et al. (2013) A G protein-biased ligand at the $\mu$-opioid receptor is potently analgesic with reduced gastrointestinal and respiratory dysfunction compared with morphine. J Pharmacol Exp Ther 344: 708-717.

de Win MM, Reneman L, Reitsma JB, den Heeten GJ, Booij J, and van den Brink W (2004) Mood disorders and serotonin transporter density in ecstasy users--the influence of long-term abstention, dose, and gender. Psychopharmacology (Berl) 173: 376-382.

Dhir A and Kulkarni SK (2007) Involvement of sigma-1 receptor modulation in the antidepressant action of venlafaxine. Neurosci Lett 420:204-208.

Diaz PM, Ngai SH, and Costa E (1968) Factors modulating brain serotonin turnover, in Advances in Pharmacology (Garattini S and Shore PA eds) pp 75-92, Academic Press, New York.

Díaz-Mataix L, Scorza MC, Bortolozzi A, Toth M, Celada P, and Artigas F (2005) Involvement of 5-HT1A receptors in prefrontal cortex in the modulation of dopaminergic activity: role in atypical antipsychotic action. J Neurosci 25:10831-10843.

Dinis-Oliveira RJ, Pereira CL, and da Silva DD (2019) Pharmacokinetic and pharmacodynamic aspects of peyote and mescaline: clinical and forensic repercussions. Curr Mol Pharmacol 12:184-194.

Diniz BS, Teixeira AL, Talib LL, Mendonca VA, Gattaz WF, and Forlenza OV (2010) Serum brain-derived neurotrophic factor level is reduced in antidepressant-free patients with late-life depression. World J Biol Psychiatry 11:550-555.

Doak GJ and Duke PC (1993) Oral clonidine premedication attenuates the haemodynamic effects associated with ketamine anaesthetic induction in humans. Can $J$ Anaesth 40:612-618.

Doblin R, Greer G, Holland J, Jerome L, Mithoefer MC, and Sessa B (2014) A reconsideration and response to Parrott AC (2013) "Human psychobiology of MDMA or 'Ecstasy': an overview of 25 years of empirical research". Hum Psychopharmacol 29:105-108.

Dolder PC, Grünblatt E, Müller F, Borgwardt SJ, and Liechti ME (2017a) A single dose of LSD does not alter gene expression of the serotonin $2 \mathrm{~A}$ receptor gene (HTR2A) or early growth response genes (EGR1-3) in healthy subjects. Front Pharmacol 8:423.

Dolder PC, Müller F, Schmid Y, Borgwardt SJ, and Liechti ME (2018) Direct comparison of the acute subjective, emotional, autonomic, and endocrine effects of MDMA, methylphenidate, and modafinil in healthy subjects. Psychopharmacology (Berl) 235:467-479.

Dolder PC, Schmid Y, Haschke M, Rentsch KM, and Liechti ME (2015) Pharmacokinetics and concentration-effect relationship of oral LSD in humans. Int $J$ Neuropsychopharmacol 19:pyv072.

Dolder PC, Schmid Y, Müller F, Borgwardt S, and Liechti ME (2016) LSD acutely impairs fear recognition and enhances emotional empathy and sociality. Neuropsychopharmacology 41:2638-2646.

Dolder PC, Schmid Y, Steuer AE, Kraemer T, Rentsch KM, Hammann F, and Liecht ME (2017b) Pharmacokinetics and pharmacodynamics of lysergic acid diethylamide in healthy subjects. Clin Pharmacokinet 56:1219-1230.

Domany Y, Shelton RC, and McCullumsmith CB (2020) Ketamine for acute suicidal ideation. An emergency department intervention: a randomized, double-blind, placebo-controlled, proof-of-concept trial. Depress Anxiety 37:224-233.

Domínguez-López S, Howell R, and Gobbi G (2012) Characterization of serotonin neurotransmission in knockout mice: implications for major depression. Rev Neurosci 23:429-443.

Dong C, Zhang JC, Yao W, Ren Q, Ma M, Yang C, Chaki S, and Hashimoto K (2017) Rapid and sustained antidepressant action of the mglu2/3 receptor antagonist mgs0039 in the social defeat stress model: comparison with ketamine. Int $J$ Neuropsychopharmacol 20:228-236.

dos Santos RG, Osorio FL, Crippa JAS, and Hallak JEC (2017) Anxiety, panic, and hopelessness during and after ritual ayahuasca intake in a woman with generalized anxiety disorder: a case report. J Psychedelic Stud 1:35-39.

Dos Santos RG, Bouso JC, and Hallak JEC (2017) Ayahuasca, dimethyltryptamine, and psychosis: a systematic review of human studies. Ther Adv Psychopharmacol 7:141-157.

Dos Santos RG, Grasa E, Valle M, Ballester MR, Bouso JC, Nomdedéu JF, Homs R, Barbanoj MJ, and Riba J (2012) Pharmacology of ayahuasca administered in two repeated doses. Psychopharmacology (Berl) 219:1039-1053.

Dowlati Y, Herrmann N, Swardfager W, Liu H, Sham L, Reim EK, and Lanctôt KI (2010) A meta-analysis of cytokines in major depression. Biol Psychiatry 67:446-457.

Dragan WL and Oniszczenko W (2009) The association between dopamine D4 receptor exon III polymorphism and intensity of PTSD symptoms among flood survivors. Anxiety Stress Coping 22:483-495.

Dremencov E, El Mansari M, and Blier P (2009) Effects of sustained serotonin reuptake inhibition on the firing of dopamine neurons in the rat ventral tegmental area. J Psychiatry Neurosci 34:223-229.

Drury SS, Theall KP, Keats BJ, and Scheeringa M (2009) The role of the dopamine transporter (DAT) in the development of PTSD in preschool children. J Trauma Stress 22:534-539.

D'Sa C and Duman RS (2002) Antidepressants and neuroplasticity. Bipolar Disord 4: 183-194.

Duclot F and Kabbaj M (2017) The role of early growth response 1 (EGR1) in brain plasticity and neuropsychiatric disorders. Front Behav Neurosci 11:35.

Duerler P, Schilbach L, Stämpfli P, Vollenweider FX, and Preller KH (2020) LSDinduced increases in social adaptation to opinions similar to one's own are associated with stimulation of serotonin receptors. Sci Rep 10:12181.

du Jardin KG, Liebenberg N, Cajina M, Müller HK, Elfving B, Sanchez C, and Wegener G (2018) $S$-ketamine mediates its acute and sustained antidepressant-like activity through a $5-\mathrm{HT}_{1 \mathrm{~B}}$ receptor dependent mechanism in a genetic rat model of depression. Front Pharmacol 8:978. 
Duman RS (2002) Pathophysiology of depression: the concept of synaptic plasticity. Eur Psychiatry 17 (Suppl 3):306-310.

Duman RS, Aghajanian GK, Sanacora G, and Krystal JH (2016) Synaptic plasticity and depression: new insights from stress and rapid-acting antidepressants. Nat Med 22:238-249.

Duman RS, Heninger GR, and Nestler EJ (1994) Molecular psychiatry. Adaptations of receptor-coupled signal transduction pathways underlying stress- and druginduced neural plasticity. J Nerv Ment Dis 182:692-700.

Dumont GJ, Sweep FC, van der Steen R, Hermsen R, Donders AR, Touw DJ, van Gerven JM, Buitelaar JK, and Verkes RJ (2009) Increased oxytocin concentrations and prosocial feelings in humans after ecstasy (3,4-methylenedioxymethamphetamine) administration. Soc Neurosci 4:359-366.

Dunlop BW and Wong A (2019) The hypothalamic-pituitary-adrenal axis in PTSD: pathophysiology and treatment interventions. Prog Neuropsychopharmacol Biol Psychiatry 89:361-379.

Dunn AJ and Swiergiel AH (2008) The role of corticotropin-releasing factor and noradrenaline in stress-related responses, and the inter-relationships between the two systems. Eur J Pharmacol 583:186-193.

Dunn HA, Orlandi C, and Martemyanov KA (2019) Beyond the ligand: extracellular and transcellular $\mathrm{g}$ protein-coupled receptor complexes in physiology and pharmacology. Pharmacol Rev 71:503-519.

Durkin S, Prendergast A, and Harkin A (2008) Reduced efficacy of fluoxetine following MDMA ("Ecstasy")-induced serotonin loss in rats. Prog Neuropsychopharmacol Biol Psychiatry 32:1894-1901.

Earley B, Burke M, Leonard BE, Gouret CJ, and Junien JL (1991) Evidence for an anti-amnesic effect of JO 1784 in the rat: a potent and selective ligand for the sigma receptor. Brain Res 546:282-286.

Ebert B, Mikkelsen S, Thorkildsen C, and Borgbjerg FM (1997) Norketamine, the main metabolite of ketamine, is a non-competitive NMDA receptor antagonist in the rat cortex and spinal cord. Eur $J$ Pharmacol 333:99-104.

Egan CT, Herrick-Davis K, Miller K, Glennon RA, and Teitler M (1998) Agonist activity of LSD and lisuride at cloned 5HT2A and 5HT2C receptors. Psychopharmacology (Berl) 136:409-414.

Elangbam CS, Job LE, Zadrozny LM, Barton JC, Yoon LW, Gates LD, and Slocum N (2008) 5-hydroxytryptamine (5HT)-induced valvulopathy: compositional valvular alterations are associated with $5 \mathrm{HT} 2 \mathrm{~B}$ receptor and $5 \mathrm{HT}$ transporter transcript changes in Sprague-Dawley rats. Exp Toxicol Pathol 60:253-262.

El Iskandrani KS, Oosterhof CA, El Mansari M, and Blier P (2015) Impact of subanesthetic doses of ketamine on AMPA-mediated responses in rats: an in vivo electrophysiological study on monoaminergic and glutamatergic neurons. J Psychopharmacol 29:792-801.

Ener RA, Meglathery SB, Van Decker WA, and Gallagher RM (2003) Serotonin syndrome and other serotonergic disorders. Pain Med 4:63-74.

Erlander MG, Lovenberg TW, Baron BM, de Lecea L, Danielson PE, Racke M, Slone AL, Siegel BW, Foye PE, Cannon K, et al. (1993) Two members of a distinct subfamily of 5-hydroxytryptamine receptors differentially expressed in rat brain. Proc Natl Acad Sci USA 90:3452-3456.

Evans JW, Lally N, An L, Li N, Nugent AC, Banerjee D, Snider SL, Shen J, Roiser JP, and Zarate CA Jr (2018a) $7 \mathrm{~T}^{1} \mathrm{H}-\mathrm{MRS}$ in major depressive disorder: a Ketamine Treatment Study. Neuropsychopharmacology 43:1908-1914.

Evans JW, Szczepanik J, Brutsché N, Park LT, Nugent AC, and Zarate CA Jr (2018b) Default mode connectivity in major depressive disorder measured up to 10 Days after ketamine administration. Biol Psychiatry 84:582-590.

Evans WE and McLeod HL (2003) Pharmacogenomics--drug disposition, drug targets, and side effects. $N$ Engl J Med 348:538-549.

Evoniuk GE, Hertzman RP, and Skolnick P (1991) A rapid method for evaluating the behavioral effects of phencyclidine-like dissociative anesthetics in mice. Psychopharmacology (Berl) 105:125-128.

Fadiman J and Korb S (2019) Might microdosing psychedelics be safe and beneficial? An initial exploration. J Psychoactive Drugs 51:118-122.

Family N, Maillet EL, Williams LTJ, Krediet E, Carhart-Harris RL, Williams TM, Nichols CD, Goble DJ, and Raz S (2020) Safety, tolerability, pharmacokinetics, and pharmacodynamics of low dose lysergic acid diethylamide (LSD) in healthy older volunteers. Psychopharmacology (Berl) 237:841-853.

Fantegrossi WE, Gray BW, Bailey JM, Smith DA, Hansen M, and Kristensen JL (2015) Hallucinogen-like effects of 2-([2-(4-cyano-2,5-dimethoxyphenyl) ethylamino]methyl)phenol (25CN-NBOH), a novel N-benzylphenethylamine with 100 fold selectivity for 5- $\mathrm{HT}_{2} \mathrm{~A}$ receptors, in mice. Psychopharmacology (Berl) 232 1039-1047.

Fantegrossi WE, Woods JH, and Winger G (2004) Transient reinforcing effects of phenylisopropylamine and indolealkylamine hallucinogens in rhesus monkeys. Behav Pharmacol 15:149-157.

Fava M, Freeman MP, Flynn M, Judge H, Hoeppner BB, Cusin C, Ionescu DF, Mathew SJ, Chang LC, Iosifescu DV, et al. (2020) Double-blind, placebo-controlled, dose-ranging trial of intravenous ketamine as adjunctive therapy in treatmentresistant depression (TRD) [published correction appears in Mol Psychiatry (2020) 25:1604]. Mol Psychiatry 25:1592-1603.

Feder A, Parides MK, Murrough JW, Perez AM, Morgan JE, Saxena S, Kirkwood K, Aan Het Rot M, Lapidus KA, Wan LB, et al. (2014) Efficacy of intravenous ketamine for treatment of chronic posttraumatic stress disorder: a randomized clinical trial. JAMA Psychiatry 71:681-688.

Feduccia AA, Jerome L, Yazar-Klosinski B, Emerson A, Mithoefer MC, and Doblin R (2019) Breakthrough for trauma treatment: safety and efficacy of mdma-assisted psychotherapy compared to paroxetine and sertraline. Front Psychiatry 10:650.

Feduccia AA and Mithoefer MC (2018) MDMA-assisted psychotherapy for PTSD: are memory reconsolidation and fear extinction underlying mechanisms?. Prog Neuropsychopharmacol Biol Psychiatry 84:221-228.

Felsing DE, Anastasio NC, Miszkiel JM, Gilbertson SR, Allen JA, and Cunningham KA (2018) Biophysical validation of serotonin 5-HT2A and 5-HT2C receptor interaction. PLoS One 13:e0203137.
Feltmann K, Borroto-Escuela DO, Rüegg J, Pinton L, de Oliveira Sergio T, Narváez M, Jimenez-Beristain A, Ekström T.J, Fuxe K, and Steensland P (2018) Effects of long-term alcohol drinking on the dopamine $\mathrm{d} 2$ receptor: gene expression and heteroreceptor complexes in the striatum in rats. Alcohol Clin Exp Res 42:338-351.

Ferrarelli F and Tononi G (2011) The thalamic reticular nucleus and schizophrenia. Schizophr Bull 37:306-315.

Ferrarelli F and Tononi G (2017) Reduced sleep spindle activity point to a TRN-MD thalamus-PFC circuit dysfunction in schizophrenia. Schizophr Res 180:36-43.

Ferreira RCM, Castor MGM, Piscitelli F, Di Marzo V, Duarte IDG, and Romero TRL (2018) The involvement of the endocannabinoid system in the peripheral antinociceptive action of ketamine. J Pain 19:487-495.

Finnegan KT and Taraska T (1996) Effects of glutamate antagonists on methamphetamine and 3,4-methylenedioxymethamphetamine-induced striatal dopamine release in vivo. J Neurochem 66:1949-1958.

Fiorella D, Helsley S, Lorrain DS, Rabin RA, and Winter JC (1995) The role of the 5HT2A and 5-HT2C receptors in the stimulus effects of hallucinogenic drugs. III: the mechanistic basis for supersensitivity to the LSD stimulus following serotonin depletion. Psychopharmacology (Berl) 121:364-372.

Fishback JA, Robson MJ, Xu YT, and Matsumoto RR (2010) Sigma receptors: potential targets for a new class of antidepressant drug. Pharmacol Ther 127: 271-282.

Fishman AP (1999) Aminorex to fen/phen: an epidemic foretold. Circulation 99: 156-161.

Flanagan TW and Nichols CD (2018) Psychedelics as anti-inflammatory agents. Int Rev Psychiatry 30:363-375.

Flanagan TW, Sebastian MN, Battaglia DM, Foster TP, Cormier SA, and Nichols CD (2019a) $5-\mathrm{HT}_{2}$ receptor activation alleviates airway inflammation and structura remodeling in a chronic mouse asthma model. Life Sci 236:116790.

Flanagan TW, Sebastian MN, Battaglia DM, Foster TP, Maillet EL, and Nichols CD (2019b) Activation of $5-\mathrm{HT}_{2}$ receptors reduces inflammation in vascular tissue and cholesterol levels in high-fat diet-fed apolipoprotein E knockout mice. Sci Rep $\mathbf{9}$ : 13444

Flint J and Kendler KS (2014) The genetics of major depression. Neuron 81:1214.

Flood P and Krasowski MD (2000) Intravenous anesthetics differentially modulate ligand-gated ion channels. Anesthesiology 92:1418-1425.

Fogarty MJ, Noakes PG, and Bellingham MC (2015) Motor cortex layer V pyramidal neurons exhibit dendritic regression, spine loss, and increased synaptic excitation in the presymptomatic hSOD1(G93A) mouse model of amyotrophic lateral sclerosis. $J$ Neurosci 35:643-647.

Foldi CJ, Liknaitzky P, Williams M, and Oldfield BJ (2020) Rethinking therapeutic strategies for anorexia nervosa: insights from psychedelic medicine and animal models. Front Neurosci 14:43.

Fontanilla D, Johannessen M, Hajipour AR, Cozzi NV, Jackson MB, and Ruoho AE (2009) The hallucinogen N,N-dimethyltryptamine (DMT) is an endogenous sigma-1 receptor regulator. Science 323:934-937.

Forstmann M and Sagioglou C (2017) Lifetime experience with (classic) psychedelics predicts pro-environmental behavior through an increase in nature relatedness. J Psychopharmacol 31:975-988.

Franceschi C, Garagnani P, Parini P, Giuliani C, and Santoro A (2018) Inflammaging: a new immune-metabolic viewpoint for age-related diseases. Nat Rev Endocrinol 14:576-590.

Francois A, Low SA, Sypek EI, Christensen AJ, Sotoudeh C, Beier KT, Ramakrishnan C, Ritola KD, Sharif-Naeini R, Deisseroth K, et al. (2017) A brainstemspinal cord inhibitory circuit for mechanical pain modulation by GABA and enkephalins. Neuron 93:822-839.e6.

Franks NP and Lieb WR (1994) Molecular and cellular mechanisms of general anaesthesia. Nature 367:607-614.

Fraser CM and Venter JC (1990) Beta-adrenergic receptors. Relationship of primary structure, receptor function, and regulation. Am Rev Respir Dis 141:S22-S30.

Frecska E, Bokor P, and Winkelman M (2016) The therapeutic potentials of ayahuasca: possible effects against various diseases of civilization. Front Pharmacol $\mathbf{7}$ : 35 .

Frecska E, Szabo A, Winkelman MJ, Luna LE, and McKenna DJ (2013) A possibly sigma-1 receptor mediated role of dimethyltryptamine in tissue protection, regeneration, and immunity. $J$ Neural Transm (Vienna) 120:1295-1303.

Freedman DX (1961) Effects of LSD-25 on brain serotonin. J Pharmacol Exp Ther 134:160-166.

Froese T, Leenen I, and Palenicek T (2018) A role for enhanced functions of sleep in psychedelic therapy?. Adapt Behav 26:129-135.

Fujimoto M, Hayashi T, Urfer R, Mita S, and Su TP (2012) Sigma-1 receptor chaperones regulate the secretion of brain-derived neurotrophic factor. Synapse 66:630-639.

Fuxe K, Holmstedt B, and Jonsson G (1972) Effects of 5-methoxy-N,N-dimethyltryptamine on central monoamine neurons. Eur J Pharmacol 19:25-34

Gaine SP, Rubin LJ, Kmetzo JJ, Palevsky HI, and Traill TA (2000) Recreational use of aminorex and pulmonary hypertension. Chest 118:1496-1497.

Galvão ACM, de Almeida RN, Silva EADS, Freire FAM, Palhano-Fontes F, Onias H, Arcoverde E, Maia-de-Oliveira JP, de Araújo DB, Lobão-Soares B, et al. (2018) Cortisol modulation by ayahuasca in patients with treatment resistant depression and healthy controls. Front Psychiatry 9:185.

Galvão-Coelho NL, de Menezes Galvão AC, de Almeida RN, Palhano-Fontes $\mathrm{F}$, Campos Braga I, Lobão Soares B, Maia-de-Oliveira JP, Perkins D, Sarris J, and de Araujo DB (2020) Changes in inflammatory biomarkers are related to the antidepressant effects of Ayahuasca. J Psychopharmacol 34:1125-1133.

Gambelunghe C, Aroni K, Rossi R, Moretti L, and Bacci M (2008) Identification of N,N-dimethyltryptamine and beta-carbolines in psychotropic ayahuasca beverage. Biomed Chromatogr 22:1056-1059.

García-Pardo MP, Escobar-Valero C, Rodríguez-Arias M, Miñarro J, and Aguilar MA (2015) Involvement of NMDA glutamate receptors in the acquisition and reinstatement of the conditioned place preference induced by MDMA. Behav Pharmacol 26:411-417. 
García-Pardo MP, Miñarro J, and Aguilar MA (2018) Role of AMPA glutamate receptors in the conditioned rewarding effects of MDMA in mice. Behav Brain Res 347:57-60.

García-Pardo MP, Miñarro J, Llansola M, Felipo V, and Aguilar MA (2019) Role of NMDA and AMPA glutamatergic receptors in the effects of social defeat on the rewarding properties of MDMA in mice. Eur J Neurosci 50:2623-2634.

Garcia-Romeu A, Davis AK, Erowid E, Erowid F, Griffiths RR, and Johnson MW (2020) Persisting reductions in cannabis, opioid, and stimulant misuse after naturalistic psychedelic use: an online survey. Front Psychiatry 10:955

Garcia-Romeu A, Davis AK, Erowid F, Erowid E, Griffiths RR, and Johnson MW (2019) Cessation and reduction in alcohol consumption and misuse after psychedelic use. J Psychopharmacol 33:1088-1101.

Garcia-Romeu A, Griffiths RR, and Johnson MW (2014) Psilocybin-occasioned mystical experiences in the treatment of tobacco addiction. Curr Drug Abuse Rev 7 : $157-164$.

Gasser P, Holstein D, Michel Y, Doblin R, Yazar-Klosinski B, Passie T, and Brenneisen R (2014) Safety and efficacy of lysergic acid diethylamide-assisted psychotherapy for anxiety associated with life-threatening diseases. J Nerv Ment Dis 202:513-520.

Gasser P, Kirchner K, and Passie T (2015) LSD-assisted psychotherapy for anxiety associated with a life-threatening disease: a qualitative study of acute and sustained subjective effects. J Psychopharmacol 29:57-68.

Gerhard DM, Pothula S, Liu RJ, Wu M, Li XY, Girgenti MJ, Taylor SR, Duman CH, Delpire E, Picciotto M, et al. (2020) GABA interneurons are the cellular trigger for ketamine's rapid antidepressant actions. $J$ Clin Invest 130:1336-1349.

Gewirtz JC and Marek GJ (2000) Behavioral evidence for interactions between a hallucinogenic drug and group II metabotropic glutamate receptors. Neuropsychopharmacology 23:569-576.

Geyer MA and Ellenbroek B (2003) Animal behavior models of the mechanisms underlying antipsychotic atypicality. Prog Neuropsychopharmacol Biol Psychiatry 27:1071-1079.

Geyer MA, Gordon J, and Adams LM (1985) Behavioral effects of xylamine-induced depletions of brain norepinephrine: interaction with LSD. Pharmacol Biochem Behav 23:619-625.

Geyer MA and Vollenweider FX (2008) Serotonin research: contributions to understanding psychoses. Trends Pharmacol Sci 29:445-453.

Giannini AJ, Nageotte C, Loiselle RH, Malone DA, and Price WA (1984-1985) Comparison of chlorpromazine, haloperidol and pimozide in the treatment of phencyclidine psychosis: DA-2 receptor specificity. $J$ Toxicol Clin Toxicol 22 $573-579$.

Gigliucci V, O’Dowd G, Casey S, Egan D, Gibney S, and Harkin A (2013) Ketamine elicits sustained antidepressant-like activity via a serotonin-dependent mechanism. Psychopharmacology (Berl) 228:157-166.

Gillis A, Gondin AB, Kliewer A, Sanchez J, Lim HD, Alamein C, Manandhar P, Santiago M, Fritzwanker S, Schmiedel F, et al. (2020) Low intrinsic efficacy for G protein activation can explain the improved side effect profiles of new opioid agonists. Sci Signal 13:eaaz3140.

Girn M, Mills C, Roseman L, Carhart-Harris RL, and Christoff K (2020) Updating the dynamic framework of thought: creativity and psychedelics. Neuroimage 213: 116726.

Glennon RA and Rosecrans JA (1982) Indolealkylamine and phenalkylamine hallucinogens: a brief overview. Neurosci Biobehav Rev 6:489-497.

Glennon RA, Titeler M, and McKenney JD (1984) Evidence for 5-HT2 involvement in the mechanism of action of hallucinogenic agents. Life Sci 35:2505-2511.

Global Burden of Disease Study 2013 Collaborators (2015) Global, regional, and national incidence, prevalence, and years lived with disability for 301 acute and chronic diseases and injuries in 188 countries, 1990-2013: a systematic analysis for the Global Burden of Disease Study 2013. Lancet 386:743-800.

Gobbi G and Blier P (2005) Effect of neurokinin-1 receptor antagonists on serotoninergic, noradrenergic and hippocampal neurons: comparison with antidepressant drugs. Peptides 26:1383-1393.

Gobbi G, Ghabrash MF, Nuñez N, Tabaka J, Di Sante J, Saint-Laurent M, Vida S, Kolivakis T, Low N, Cervantes P, et al. (2018) Antidepressant combination versus antidepressants plus second-generation antipsychotic augmentation in treatmentresistant unipolar depression. Int Clin Psychopharmacol 33:34-43.

Goitia B, Rivero-Echeto MC, Weisstaub NV, Gingrich JA, Garcia-Rill E, Bisagno V, and Urbano FJ (2016) Modulation of GABA release from the thalamic reticular nucleus by cocaine and caffeine: role of serotonin receptors. J Neurochem 136 $526-535$.

Gola H, Engler H, Sommershof A, Adenauer H, Kolassa S, Schedlowski M, Groettrup M, Elbert T, and Kolassa IT (2013) Posttraumatic stress disorder is associated with an enhanced spontaneous production of pro-inflammatory cytokines by peripheral blood mononuclear cells. BMC Psychiatry 13:40

Goldberger L (1966) Cognitive test performance under LSD-25, placebo and isolation. $J$ Nerv Ment Dis 142:4-9.

Goldman S, Galarneau D, and Friedman R (2007) New onset LSD flashback syndrome triggered by the initiation of SSRIs. Ochsner $J$ 7:37-39.

Goldsmith DR, Rapaport MH, and Miller BJ (2016) A meta-analysis of blood cytokine network alterations in psychiatric patients: comparisons between schizophrenia, bipolar disorder and depression. Mol Psychiatry 21:1696-1709.

Goll Y, Atlan G, and Citri A (2015) Attention: the claustrum. Trends Neurosci 38: 486-495

Gomes I, Sierra S, Lueptow L, Gupta A, Gouty S, Margolis EB, Cox BM, and Devi LA (2020) Biased signaling by endogenous opioid peptides. Proc Natl Acad Sci USA 117:11820-11828.

Gómez-Gil E, Gastó C, Carretero M, Díaz-Ricart M, Salamero M, Navinés R, and Escolar G (2004) Decrease of the platelet 5-HT2A receptor function by long-term imipramine treatment in endogenous depression. Hum Psychopharmacol 19:251-258.

González-Maeso J, Ang RL, Yuen T, Chan P, Weisstaub NV, López-Giménez JF, Zhou M, Okawa Y, Callado LF, Milligan G, et al. (2008) Identification of a serotonin/glutamate receptor complex implicated in psychosis. Nature 452:93-97.
González-Maeso J, Weisstaub NV, Zhou M, Chan P, Ivic L, Ang R, Lira A, BradleyMoore M, Ge Y, Zhou Q, et al. (2007) Hallucinogens recruit specific cortical 5HT(2A) receptor-mediated signaling pathways to affect behavior. Neuron $\mathbf{5 3}$ 439-452.

González-Maeso J, Yuen T, Ebersole BJ, Wurmbach E, Lira A, Zhou M, Weisstaub N, Hen R, Gingrich JA, and Sealfon SC (2003) Transcriptome fingerprints distinguish hallucinogenic and nonhallucinogenic 5-hydroxytryptamine $2 \mathrm{~A}$ receptor agonist effects in mouse somatosensory cortex. J Neurosci 23:8836-8843.

Goodwin AK (2016) An intravenous self-administration procedure for assessing the reinforcing effects of hallucinogens in nonhuman primates. $J$ Pharmacol Toxicol Methods 82:31-36.

Gorman I, Belser AB, Jerome L, Hennigan C, Shechet B, Hamilton S, Yazar-Klosinski B, Emerson A, and Feduccia AA (2020) Posttraumatic growth after MDMAassisted psychotherapy for posttraumatic stress disorder. J Trauma Stress 33: 161-170.

Gough B, Ali SF, Slikker W Jr, and Holson RR (1991) Acute effects of 3,4-methylenedioxymethamphetamine (MDMA) on monoamines in rat caudate. Pharmacol Biochem Behav 39:619-623.

Gouzoulis-Mayfrank E, Thelen B, Habermeyer E, Kunert HJ, Kovar KA, Lindenblatt H, Hermle L, Spitzer M, and Sass H (1999) Psychopathological, neuroendocrine and autonomic effects of 3,4-methylenedioxyethylamphetamine (MDE), psilocybin and d-methamphetamine in healthy volunteers. Results of an experimental doubleblind placebo-controlled study. Psychopharmacology (Berl) 142:41-50.

Grace AA (2016) Dysregulation of the dopamine system in the pathophysiology of schizophrenia and depression. Nat Rev Neurosci 17:524-532.

Graham JR (1967) Cardiac and pulmonary fibrosis during methysergide therapy for headache. Trans Am Clin Climatol Assoc 78:79-92.

Graham JR, Suby HI, LeCompte PR, and Sadowsky NL (1966) Fibrotic disorders associated with methysergide therapy for headache. $N$ Engl J Med 274:359-368.

Granado N, Ares-Santos S, Oliva I, O'Shea E, Martin ED, Colado MI, and Moratalla R (2011) Dopamine D2-receptor knockout mice are protected against dopaminergic neurotoxicity induced by methamphetamine or MDMA. Neurobiol Dis 42:391-403.

Graudins A, Stearman A, and Chan B (1998) Treatment of the serotonin syndrome with cyproheptadine. J Emerg Med 16:615-619.

Gray JA and Roth BL (2001) Paradoxical trafficking and regulation of 5-HT(2A) receptors by agonists and antagonists. Brain Res Bull 56:441-451.

Green AR, Mechan AO, Elliott JM, O'Shea E, and Colado MI (2003) The pharmacology and clinical pharmacology of 3,4-methylenedioxymethamphetamine (MDMA, "ecstasy"). Pharmacol Rev 55:463-508.

Green WJ (1965) The effect of LSD on the sleep-dream cycle. An exploratory study. $J$ Nerv Ment Dis 140:417-426.

Gresch PJ, Smith RL, Barrett RJ, and Sanders-Bush E (2005) Behavioral tolerance to lysergic acid diethylamide is associated with reduced serotonin-2A receptor signaling in rat cortex. Neuropsychopharmacology 30:1693-1702.

Griffiths R, Richards W, Johnson M, McCann U, and Jesse R (2008) Mysticaltype experiences occasioned by psilocybin mediate the attribution of personal meaning and spiritual significance 14 months later. $J$ Psychopharmacol 22: 621-632.

Griffiths RR, Johnson MW, Carducci MA, Umbricht A, Richards WA, Richards BD Cosimano MP, and Klinedinst MA (2016) Psilocybin produces substantial and sustained decreases in depression and anxiety in patients with life-threatening cancer: a randomized double-blind trial. J Psychopharmacol 30:1181-1197.

Griffiths RR, Johnson MW, Richards WA, Richards BD, Jesse R, MacLean KA, Barrett FS, Cosimano MP, and Klinedinst MA (2018) Psilocybin-occasioned mystical-type experience in combination with meditation and other spiritual practices produces enduring positive changes in psychological functioning and in trait measures of prosocial attitudes and behaviors. J Psychopharmacol 32: $49-69$

Griffiths RR, Johnson MW, Richards WA, Richards BD, McCann U, and Jesse R (2011) Psilocybin occasioned mystical-type experiences: immediate and persisting dose-related effects. Psychopharmacology (Berl) 218:649-665.

Griffiths RR, Richards WA, McCann U, and Jesse R (2006) Psilocybin can occasion mystical-type experiences having substantial and sustained personal meaning and spiritual significance. Psychopharmacology (Berl) 187:268-283, discussion 284-292.

Grob CS, Danforth AL, Chopra GS, Hagerty M, McKay CR, Halberstadt AL, and Greer GR (2011) Pilot study of psilocybin treatment for anxiety in patients with advanced-stage cancer. Arch Gen Psychiatry 68:71-78.

Groh G and Lemieux M (1968) The effect of LSD-25 on spider web formation. Int $J$ Addict 3:41-53.

Grunebaum MF, Galfalvy HC, Choo TH, Keilp JG, Moitra VK, Parris MS, Marver JE Burke AK, Milak MS, Sublette ME, et al. (2018) Ketamine for rapid reduction of suicidal thoughts in major depression: a midazolam-controlled randomized clinical trial. Am J Psychiatry 175:327-335.

Gudelsky GA and Nash JF (1996) Carrier-mediated release of serotonin by 3,4 methylenedioxymethamphetamine: implications for serotonin-dopamine interactions. J Neurochem 66:243-249.

Gudelsky GA, Yamamoto BK, and Nash JF (1994) Potentiation of 3,4-methylenedioxymethamphetamine-induced dopamine release and serotonin neurotoxicity by 5-HT2 receptor agonists. Eur J Pharmacol 264:325-330.

Guevara-Lora I, Niewiarowska-Sendo A, Polit A, and Kozik A (2016) Hypothetical orchestrated cooperation between dopaminergic and kinin receptors for the regulation of common functions. Acta Biochim Pol 63:387-396.

Guiard BP, El Mansari M, Merali Z, and Blier P (2008) Functional interactions between dopamine, serotonin and norepinephrine neurons: an in-vivo electrophysiological study in rats with monoaminergic lesions. Int $J$ Neuropsychopharmacol 11:625-639.

Gurtner HP (1985) Aminorex and pulmonary hypertension. A review. Cor Vasa 27: $160-171$.

Gururajan A, Clarke G, Dinan TG, and Cryan JF (2016) Molecular biomarkers of depression. Neurosci Biobehav Rev 64:101-133. 
Gustafsson BI, Tømmerås K, Nordrum I, Loennechen JP, Brunsvik A, Solligård E, Fossmark R, Bakke I, Syversen U, and Waldum H (2005) Long-term serotonin administration induces heart valve disease in rats. Circulation 111:1517-1522.

Haddjeri N, Blier P, and de Montigny C (1998) Long-term antidepressant treatments result in a tonic activation of forebrain 5-HT1A receptors. $J$ Neurosci 18 $10150-10156$

Haden M and Woods B (2020) LSD overdoses: three case reports. J Stud Alcohol Drugs 81:115-118.

Hagino Y, Takamatsu Y, Yamamoto H, Iwamura T, Murphy DL, Uhl GR, Sora I, and Ikeda K (2011) Effects of MDMA on extracellular dopamine and serotonin levels in mice lacking dopamine and/or serotonin transporters. Curr Neuropharmacol 9:91-95.

Haijen ECHM, Kaelen M, Roseman L, Timmermann C, Kettner H, Russ S, Nutt D, Daws RE, Hampshire ADG, Lorenz R, et al. (2018) Predicting responses to psychedelics: a prospective study. Front Pharmacol 9:897.

Hake HS, Davis JKP, Wood RR, Tanner MK, Loetz EC, Sanchez A, Ostrovskyy M, Oleson EB, Grigsby J, Doblin R, et al. (2019) 3,4-methylenedioxymethamphetamine (MDMA) impairs the extinction and reconsolidation of fear memory in rats Physiol Behav 199:343-350.

Halberstadt AL, Chatha M, Klein AK, McCorvy JD, Meyer MR, Wagmann L, Stratford A, and Brandt SD (2020) Pharmacological and biotransformation studies of 1-acyl-substituted derivatives of d-lysergic acid diethylamide (LSD). Neuropharmacology 172:107856.

Hall AP and Henry JA (2006) Acute toxic effects of 'Ecstasy' (MDMA) and related compounds: overview of pathophysiology and clinical management. $\mathrm{Br} \mathrm{J}$ Anaesth 96:678-685.

Halpern JH, Lerner AG, and Passie T (2018) A review of hallucinogen persisting perception disorder (HPPD) and an exploratory study of subjects claiming symptoms of HPPD. Curr Top Behav Neurosci 36:333-360.

Halpern JH, Sherwood AR, Hudson JI, Yurgelun-Todd D, and Pope HG Jr (2005) Psychological and cognitive effects of long-term peyote use among Native Americans. Biol Psychiatry 58:624-631.

Hamill J, Hallak J, Dursun SM, and Baker G (2019) Ayahuasca: psychological and physiologic effects, pharmacology and potential uses in addiction and mental illness. Curr Neuropharmacol 17:108-128.

Hamilton JP, Furman DJ, Chang C, Thomason ME, Dennis E, and Gotlib IH (2011) Default-mode and task-positive network activity in major depressive disorder: implications for adaptive and maladaptive rumination. Biol Psychiatry $\mathbf{7 0}$ $327-333$.

Hamon M and Blier P (2013) Monoamine neurocircuitry in depression and strategies for new treatments. Prog Neuropsychopharmacol Biol Psychiatry 45:54-63.

Hanamsagar R, Hanke ML, and Kielian T (2012) Toll-like receptor (TLR) and inflammasome actions in the central nervous system. Trends Immunol 33:333-342.

Hansen M, Phonekeo K, Paine JS, Leth-Petersen S, Begtrup M, Bräuner-Osborne H, and Kristensen JL (2014) Synthesis and structure-activity relationships of N-benzyl phenethylamines as 5-HT2A/2C agonists. ACS Chem Neurosci 5: 243-249.

Hara K, Minami K, Ueno S, Toyohira Y, Tsutsui M, Shigematsu A, and Yanagihara N (2002) Up-regulation of noradrenaline transporter in response to prolonged exposure to ketamine. Naunyn Schmiedebergs Arch Pharmacol 365:406-412.

Hara K, Yanagihara N, Minami K, Hirano H, Sata T, Shigematsu A, and Izumi F (2000) Dual effects of intravenous anesthetics on the function of norepinephrine transporters. Anesthesiology 93:1329-1335.

Hara K, Yanagihara N, Minami K, Ueno S, Toyohira Y, Sata T, Kawamura M, Brüss M, Bönisch H, Shigematsu A, et al. (1998) Ketamine interacts with the noradrenaline transporter at a site partly overlapping the desipramine binding site. Naunyn Schmiedebergs Arch Pharmacol 358:328-333.

Harmer CJ, Duman RS, and Cowen PJ (2017) How do antidepressants work? New perspectives for refining future treatment approaches. Lancet Psychiatry 4 $409-418$.

Hartogsohn I (2018) The meaning-enhancing properties of psychedelics and their mediator role in psychedelic therapy, spirituality, and creativity. Front Neurosci 12:129

Harvey M, Sleigh J, Voss L, Bickerdike M, Dimitrov I, and Denny W (2019) KEA1010 , a ketamine ester analogue, retains analgesic and sedative potency but is devoid of Psychomimetic effects. BMC Pharmacol Toxicol 20:85.

Hasan A, Falkai P, Wobrock T, Lieberman J, Glenthoj B, Gattaz WF, Thibaut F, and Möller H-J; World Federation of Societies of Biological Psychiatry (WFSBP Task Force on Treatment Guidelines for Schizophrenia (2012) World Federation of Societies of Biological Psychiatry (WFSBP) guidelines for biological treatment of schizophrenia, part 1: update 2012 on the acute treatment of schizophrenia and the management of treatment resistance. World J Biol Psychiatry 13:318-378.

Hasan A, Falkai P, Wobrock T, Lieberman J, Glenthoj B, Gattaz WF, Thibaut F, and Möller H-J; WFSBP Task force on Treatment Guidelines for Schizophrenia (2013) World Federation of Societies of Biological Psychiatry (WFSBP) guidelines for biological treatment of schizophrenia, part 2: update 2012 on the long-term treatment of schizophrenia and management of antipsychotic-induced side effects World J Biol Psychiatry 14:2-44.

Hashimoto K (2014) The R-stereoisomer of ketamine as an alternative for ketamine for treatment-resistant major depression. Clin Psychopharmacol Neurosci 12 $72-73$

Hasler F, Grimberg U, Benz MA, Huber T, and Vollenweider FX (2004) Acute psychological and physiological effects of psilocybin in healthy humans: a double-blind, placebo-controlled dose-effect study. Psychopharmacology (Berl) 172:145-156.

Haubrich DR and Wang PFL (1977) N'N-dimethyltryptamine lowers rat brain acetylcholine and dopamine. Brain Res 131:158-161.

Hayashi T and Su TP (2007) Sigma-1 receptor chaperones at the ER-mitochondrion interface regulate $\mathrm{Ca}(2+)$ signaling and cell survival. Cell 131:596-610.

Hegadoren KM, Baker GB, and Bourin M (1999) 3,4-Methylenedioxy analogues of amphetamine: defining the risks to humans. Neurosci Biobehav Rev 23:539-553.
Heifets BD, Salgado JS, Taylor MD, Hoerbelt P, Cardozo Pinto DF, Steinberg EE Walsh JJ, Sze JY, and Malenka RC (2019) Distinct neural mechanisms for the prosocial and rewarding properties of MDMA. Sci Transl Med 11:eaaw6435.

Heim C, Newport DJ, Mletzko T, Miller AH, and Nemeroff CB (2008) The link between childhood trauma and depression: insights from HPA axis studies in humans. Psychoneuroendocrinology 33:693-710.

Hemmings SM, Martin LI, Klopper M, van der Merwe L, Aitken L, de Wit E, Black GF, Hoal EG, Walzl G, and Seedat S (2013) BDNF Val66Met and DRD2 Taq1A polymorphisms interact to influence PTSD symptom severity: a preliminary investigation in a South African population. Prog Neuropsychopharmacol Biol Psychiatry 40:273-280.

Hendricks PS, Crawford MS, Cropsey KL, Copes H, Sweat NW, Walsh Z, and Pavela $\mathrm{G}$ (2018) The relationships of classic psychedelic use with criminal behavior in the United States adult population. J Psychopharmacol 32:37-48.

Hendricks PS, Thorne CB, Clark CB, Coombs DW, and Johnson MW (2015) Classic psychedelic use is associated with reduced psychological distress and suicidality in the United States adult population. J Psychopharmacol 29:280-288.

Herbet G, Lafargue G, de Champfleur NM, Moritz-Gasser S, le Bars E, Bonnetblanc F, and Duffau H (2014) Disrupting posterior cingulate connectivity disconnects consciousness from the external environment. Neuropsychologia 56:239-244.

Herblin WF and O'Brien RD (1968) Interaction of norepinephrine with subcellular fractions of rat brain. I. Characteristics of norepinephrine uptake. Brain Res 8 : 298-309.

Herrera CG, Cadavieco MC, Jego S, Ponomarenko A, Korotkova T, and Adamantidis A (2016) Hypothalamic feedforward inhibition of thalamocortical network controls arousal and consciousness. Nat Neurosci 19:290-298.

Hibicke M, Landry AN, Kramer HM, Talman ZK, and Nichols CD (2020) Psychedelics, but not ketamine, produce persistent antidepressant-like effects in a rodent experimental system for the study of depression. ACS Chem Neurosci 11:864-871.

Hill R, Disney A, Conibear A, Sutcliffe K, Dewey W, Husbands S, Bailey C, Kelly E, and Henderson G (2018) The novel $\mu$-opioid receptor agonist PZM21 depresses respiration and induces tolerance to antinociception. $\mathrm{Br}$ J Pharmacol 175:2653-2661.

Hindmarch I and Hashimoto K (2010) Cognition and depression: the effects of fluvoxamine, a sigma-1 receptor agonist, reconsidered. Hum Psychopharmacol 25:193-200.

Hobson JA (1964) The effect of lsd on the sleep cycle of the cat. Electroencephalogr Clin Neurophysiol 17:52-56.

Höflich A, Hahn A, Küblböck M, Kranz GS, Vanicek T, Windischberger C, Saria A Kasper S, Winkler D, and Lanzenberger R (2015) Ketamine-induced modulation of the thalamo-cortical network in healthy volunteers as a model for schizophrenia Int $J$ Neuropsychopharmacol 18:pyv040.

Hofmann A, Heim R, Brack A, and Kobel H (1958) [Psilocybin, a psychotropic substance from the Mexican mushroom Psilicybe mexicana Heim]. Experientia 14: 107-109.

Hofmann A (1979) How LSD originated. J Psychedelic Drugs 11:53-60.

Hollands C, Tobin MK, Hsu M, Musaraca K, Yu TS, Mishra R, Kernie SG, and Lazarov O (2017) Depletion of adult neurogenesis exacerbates cognitive deficits in Alzheimer's disease by compromising hippocampal inhibition. Mol Neurodegener 12:64.

Hollister LE and Moore F (1967) Urinary catecholamine excretion following lysergic acid diethylamide in man. Psychopharmacology (Berl) 11:270-275.

Holsen LM, Lancaster K, Klibanski A, Whitfield-Gabrieli S, Cherkerzian S, Buka S, and Goldstein JM (2013) HPA-axis hormone modulation of stress response circuitry activity in women with remitted major depression. Neuroscience 250: $733-742$.

Holze F, Duthaler U, Vizeli P, Müller F, Borgwardt S, and Liechti ME (2019) Pharmacokinetics and subjective effects of a novel oral LSD formulation in healthy subjects. Br J Clin Pharmacol 85:1474-1483.

Holze F, Vizeli P, Müller F, Ley L, Duerig R, Varghese N, Eckert A, Borgwardt S, and Liechti ME (2020) Distinct acute effects of LSD, MDMA, and D-amphetamine in healthy subjects. Neuropsychopharmacology 45:462-471.

Homayoun H and Moghaddam B (2007) NMDA receptor hypofunction produces opposite effects on prefrontal cortex interneurons and pyramidal neurons. J Neurosc 27:11496-11500

Horsley RR, Páleníček T, Kolin J, and Valeš K (2018) Psilocin and ketamine microdosing: effects of subchronic intermittent microdoses in the elevated plus-maze in male Wistar rats. Behav Pharmacol 29:530-536.

Hotamisligil GS (2017) Inflammation, metaflammation and immunometabolic disorders. Nature 542:177-185.

House RV, Thomas PT, and Bhargava HN (1994) Immunological consequences of in vitro exposure to lysergic acid diethylamide (LSD). Immunopharmacol Immunotoxicol 16:23-40.

Howells FM, Stein DJ, and Russell VA (2012) Synergistic tonic and phasic activity of the locus coeruleus norepinephrine (LC-NE) arousal system is required for optimal attentional performance. Metab Brain Dis 27:267-274.

Hoyer D (1988) Functional correlates of serotonin 5-HT1 recognition sites. J Recept Res 8:59-81.

Hu QD, Xu LL, Gong Y, Wu GH, Wang YW, Wu SJ, Zhang Z, Mao W, Zhou YS, Li QB et al. (2018) Lysergic acid diethylamide causes photoreceptor cell damage through inducing inflammatory response and oxidative stress. Cutan Ocul Toxicol 37: 233-239.

Hu XZ, Lipsky RH, Zhu G, Akhtar LA, Taubman J, Greenberg BD, Xu K, Arnold PD, Richter MA, Kennedy JL, et al. (2006) Serotonin transporter promoter gain-of-function genotypes are linked to obsessive-compulsive disorder. Am J Hum Genet 78:815-826.

Huang MC, Chen LY, Chang HM, Liang XY, Chen CK, Cheng WJ, and Xu K (2018) Decreased blood levels of oxytocin in ketamine-dependent patients during early abstinence. Front Psychiatry 9:633.

Huff CL, Morano RL, Herman JP, Yamamoto BK, and Gudelsky GA (2016) MDMA decreases olutamic acid decarboxylase (GAD) 67-immunoreactive neurons in the hippocampus and increases seizure susceptibility: role for glutamate. Neurotoxicology 57:282-290. 
Hungen KV, Roberts S, and Hill DF (1975) Interactions between lysergic acid diethylamide and dopamine-sensitive adenylate cyclase systems in rat brain. Brain Res 94:57-66.

Hunt MJ, Kopell NJ, Traub RD, and Whittington MA (2017) Aberrant network activity in schizophrenia. Trends Neurosci 40:371-382.

Hutcheson JD, Setola V, Roth BL, and Merryman WD (2011) Serotonin receptors and heart valve disease--it was meant 2B. Pharmacol Ther 132:146-157.

Hutten NRPW, Mason NL, Dolder PC, Theunissen EL, Holze F, Liechti ME, Varghese N, Eckert A, Feilding A, Ramaekers JG, et al. (2020) Low doses of LSD acutely increase BDNF blood plasma levels in healthy volunteers. ACS Pharmacol Transl Sci, doi: 10.1021/acsptsci.0c00099.

Hutten NRPW, Mason NL, Dolder PC, Theunissen EL, Holze F, Liechti ME, Feilding A, Ramaekers JG, and Kuypers KPC (2020) Mood and cognition after administration of low LSD doses in healthy volunteers: A placebo controlled dose-effect finding study. European Neuropsychopharmacology, doi: 10.1016/j.euroneuro.2020.10.002.

Hutten NRPW, Mason NL, Dolder PC, and Kuypers KPC (2019) Motives and sideeffects of microdosing with psychedelics among users. Int $J$ Neuropsychopharmacol 22:426-434.

Hysek C, Schmid Y, Rickli A, Simmler LD, Donzelli M, Grouzmann E, and Liechti ME (2012a) Carvedilol inhibits the cardiostimulant and thermogenic effects of MDMA in humans. Br J Pharmacol 166:2277-2288.

Hysek CM, Brugger R, Simmler LD, Bruggisser M, Donzelli M, Grouzmann E, Hoener MC, and Liechti ME (2012b) Effects of the $\alpha_{2}$-adrenergic agonist clonidine on the pharmacodynamics and pharmacokinetics of 3,4-methylenedioxymethamphetamine in healthy volunteers. $J$ Pharmacol Exp Ther 340:286-294.

Hysek CM, Fink AE, Simmler LD, Donzelli M, Grouzmann E, and Liechti ME (2013) $\alpha_{1}$-Adrenergic receptors contribute to the acute effects of 3,4-methylenedioxymethamphetamine in humans. J Clin Psychopharmacol 33:658-666.

Hysek CM and Liechti ME (2012) Effects of MDMA alone and after pretreatment with reboxetine, duloxetine, clonidine, carvedilol, and doxazosin on pupillary light reflex. Psychopharmacology (Berl) 224:363-376.

Hysek CM, Simmler LD, Ineichen M, Grouzmann E, Hoener MC, Brenneisen R, Huwyler J, and Liechti ME (2011) The norepinephrine transporter inhibitor reboxetine reduces stimulant effects of MDMA ("ecstasy") in humans. Clin Phar macol Ther 90:246-255.

Hysek CM, Simmler LD, Schillinger N, Meyer N, Schmid Y, Donzelli M, Grouzmann E, and Liechti ME (2014) Pharmacokinetic and pharmacodynamic effects of methylphenidate and MDMA administered alone or in combination. Int $J$ Neuropsychopharmacol 17:371-381.

Hysek CM, Vollenweider FX, and Liechti ME (2010) Effects of a beta-blocker on the cardiovascular response to MDMA (Ecstasy). Emerg Med J 27:586-589.

Inserra A (2018) Hypothesis: the psychedelic ayahuasca heals traumatic memories via a sigma 1 receptor-mediated epigenetic-mnemonic process. Front Pharmacol $\mathbf{9}$ 330

Inserra A, Mastronardi CA, Rogers G, Licinio J, and Wong ML (2019) Neuroimmunomodulation in major depressive disorder: focus on caspase 1, inducible nitric oxide synthase, and interferon-gamma. Mol Neurobiol 56:4288-4305.

Inserra A, Rogers GB, Licinio J, and Wong ML (2018) The microbiota-inflammasome hypothesis of major depression. BioEssays 40:e1800027.

Ionescu DF, Luckenbaugh DA, Niciu MJ, Richards EM, Slonena EE, Vande Voort JL, Brutsche NE, and Zarate CA Jr (2014) Effect of baseline anxious depression on initial and sustained antidepressant response to ketamine. J Clin Psychiatry $\mathbf{7 5}$ e932-e938.

Ishikawa M, Ishiwata K, Ishii K, Kimura Y, Sakata M, Naganawa M, Oda K, Miyatake R, Fujisaki M, Shimizu E, et al. (2007) High occupancy of sigma-1 receptors in the human brain after single oral administration of fluvoxamine: a positron emission tomography study using [11C]SA4503. Biol Psychiatry 62 $878-883$

Ishikawa R, Fukushima H, Frankland PW, and Kida S (2016) Hippocampal neurogenesis enhancers promote forgetting of remote fear memory after hippocampal reactivation by retrieval. eLife $\mathbf{5}: \mathrm{e} 17464$

Ishikawa R, Uchida C, Kitaoka S, Furuyashiki T, and Kida S (2019) Improvement of PTSD-like behavior by the forgetting effect of hippocampal neurogenesis enhancer memantine in a social defeat stress paradigm. Mol Brain 12:68.

Jackson J, Karnani MM, Zemelman BV, Burdakov D, and Lee AK (2018) Inhibitory control of prefrontal cortex by the claustrum. Neuron 99:1029-1039.e4.

Jackson ME, Homayoun H, and Moghaddam B (2004) NMDA receptor hypofunction produces concomitant firing rate potentiation and burst activity reduction in the prefrontal cortex. Proc Natl Acad Sci USA 101:8467-8472.

Jakab RL and Goldman-Rakic PS (1998) 5-Hydroxytryptamine2A serotonin receptors in the primate cerebral cortex: possible site of action of hallucinogenic and antipsychotic drugs in pyramidal cell apical dendrites. Proc Natl Acad Sci USA 95:735-740.

Jarvik ME, Abramson HA, and Hirsch MW (1955) Lysergic acid diethylamide (LSD25): IV. Effect on attention and concentration. J Psychol 40:373.

Jauhar S, Veronese M, Nour MM, Rogdaki M, Hathway P, Natesan S, Turkheimer F, Stone J, Egerton A, McGuire P, et al. (2019) The effects of antipsychotic treatment on presynaptic dopamine synthesis capacity in first-episode psychosis: a positron emission tomography study. Biol Psychiatry 85:79-87.

Jeevakumar V and Kroener S (2016) Ketamine administration during the second postnatal week alters synaptic properties of fast-spiking interneurons in the medial prefrontal cortex of adult mice. Cereb Cortex 26:1117-1129.

Jefsen O, Elfving B, Wegener G, and Muller HK (2020) Transcriptional regulation in the rat prefrontal cortex and hippocampus after a single administration of psilocybin. J Psychopharmacol, doi: 10.1177/0269881120959614.

Jensen AA, Halberstadt AL, Märcher-Rørsted E, Odland AU, Chatha M, Speth N, Liebscher G, Hansen M, Bräuner-Osborne H, Palner M, et al. (2020) The selective $5-\mathrm{HT}_{2 \mathrm{~A}}$ receptor agonist $25 \mathrm{CN}-\mathrm{NBOH}$ : structure-activity relationship, in vivo pharmacology, and in vitro and ex vivo binding characteristics of $\left[{ }^{3} \mathrm{H}\right] 25 \mathrm{CN}-\mathrm{NBOH}$ Biochem Pharmacol 177:113979.
Jentsch JD and Roth RH (1999) The neuropsychopharmacology of phencyclidine: from NMDA receptor hypofunction to the dopamine hypothesis of schizophrenia. Neuropsychopharmacology 20:201-225.

Jernigan CS, Goswami DB, Austin MC, Iyo AH, Chandran A, Stockmeier CA, and Karolewicz B (2011) The mTOR signaling pathway in the prefrontal cortex is compromised in major depressive disorder. Prog Neuropsychopharmacol Biol Psychiatry 35:1774-1779.

Jiménez-Garrido DF, Gómez-Sousa M, Ona G, Dos Santos RG, Hallak JEC, AlcázarCórcoles MÁ, and Bouso JC (2020) Effects of ayahuasca on mental health and quality of life in naïve users: a longitudinal and cross-sectional study combination. Sci Rep 10:4075.

Johansen PØ and Krebs TS (2015) Psychedelics not linked to mental health problems or suicidal behavior: a population study. J Psychopharmacol 29:270-279.

Johnson JW and Kotermanski SE (2006) Mechanism of action of memantine. Curr Opin Pharmacol 6:61-67.

Johnson M, Mitros K, Stone DM, Zobrist R, Hanson GR, and Gibb JW (1992) Effect of flunarizine and nimodipine on the decrease in tryptophan hydroxylase activity induced by methamphetamine and 3,4-methylenedioxymethamphetamine. J Pharmacol Exp Ther 261:586-591.

Johnson MW, Garcia-Romeu A, Cosimano MP, and Griffiths RR (2014) Pilot study of the 5-HT2AR agonist psilocybin in the treatment of tobacco addiction. J Psychopharmacol 28:983-992.

Johnson MW, Garcia-Romeu A, and Griffiths RR (2017) Long-term follow-up of psilocybin-facilitated smoking cessation. Am J Drug Alcohol Abuse 43:55-60.

Johnson MW, Hendricks PS, Barrett FS, and Griffiths RR (2019) Classic psychedelics: an integrative review of epidemiology, therapeutics, mystical experience, and brain network function. Pharmacol Ther 197:83-102.

Johnson MW, Sewell RA, and Griffiths RR (2012) Psilocybin dose-dependently causes delayed, transient headaches in healthy volunteers. Drug Alcohol Depend 123: $132-140$

Johnstad PG (2018) Powerful substances in tiny amounts: an interview study of psychedelic microdosing. Nordisk Alkohol Nark 35:39-51.

Jones EG (2001) The thalamic matrix and thalamocortical synchrony. Trends Neurosci 24:595-601.

Jones KA, Srivastava DP, Allen JA, Strachan RT, Roth BL, and Penzes P (2009) Rapid modulation of spine morphology by the 5-HT2A serotonin receptor through kalirin-7 signaling. Proc Natl Acad Sci USA 106:19575-19580.

Kaelen M, Barrett FS, Roseman L, Lorenz R, Family N, Bolstridge M, Curran HV, Feilding A, Nutt DJ, and Carhart-Harris RL (2015) LSD enhances the emotional response to music. Psychopharmacology (Berl) 232:3607-3614

Kaelen M, Roseman L, Kahan J, Santos-Ribeiro A, Orban C, Lorenz R, Barrett FS, Bolstridge M, Williams T, Williams L, et al. (2016) LSD modulates music-induced imagery via changes in parahippocampal connectivity. Eur Neuropsychopharmacol 26:1099-1109.

Kalant H (2001) The pharmacology and toxicology of "ecstasy" (MDMA) and related drugs. CMAJ 165:917-928.

Kallarackal AJ, Kvarta MD, Cammarata E, Jaberi L, Cai X, Bailey AM, and Thompson SM (2013) Chronic stress induces a selective decrease in AMPA receptor-mediated synaptic excitation at hippocampal temporoammonic-CA1 synapses. J Neurosci 33:15669-15674.

Kankaanpää A, Meririnne E, Lillsunde P, and Seppälä T (1998) The acute effects of amphetamine derivatives on extracellular serotonin and dopamine levels in rat nucleus accumbens. Pharmacol Biochem Behav 59:1003-1009.

Kappelmann N, Lewis G, Dantzer R, Jones PB, and Khandaker GM (2018) Antidepressant activity of anti-cytokine treatment: a systematic review and metaanalysis of clinical trials of chronic inflammatory conditions. Mol Psychiatry 23: 335-343.

Kapur S and Remington G (1996) Serotonin-dopamine interaction and its relevance to schizophrenia. Am J Psychiatry 153:466-476.

Kapur S and Seeman P (2002) NMDA receptor antagonists ketamine and PCP have direct effects on the dopamine $\mathrm{D}(2)$ and serotonin 5-HT(2)receptors-implications for models of schizophrenia. Mol Psychiatry 7:837-844.

Karaki S, Becamel C, Murat S, Mannoury la Cour C, Millan MJ, Prézeau L, Bockaert J, Marin P, and Vandermoere F (2014) Quantitative phosphoproteomics unravels biased phosphorylation of serotonin 2A receptor at Ser280 by hallucinogenic versus nonhallucinogenic agonists. Mol Cell Proteomics 13:1273-1285.

Karg K, Burmeister M, Shedden K, and Sen S (2011) The serotonin transporter promoter variant (5-HTTLPR), stress, and depression meta-analysis revisited evidence of genetic moderation. Arch Gen Psychiatry 68:444-454.

Kargieman L, Santana N, Mengod G, Celada P, and Artigas F (2007) Antipsychotic drugs reverse the disruption in prefrontal cortex function produced by NMDA receptor blockade with phencyclidine. Proc Natl Acad Sci USA 104:14843-14848.

Kasa K, Otsuki S, Yamamoto M, Sato M, Kuroda H, and Ogawa N (1982) Cerebrospinal fluid gamma-aminobutyric acid and homovanillic acid in depressive disorders. Biol Psychiatry 17:877-883.

Katz RI and Kopin IJ (1969) Release of norepinephrine-3H and serotonin-3H evoked from brain slices by electrical-field stimulation-calcium dependency and the effects of lithium, ouabain and tetrodotoxin. Biochem Pharmacol 18:1835-1839.

Kauer-Sant'Anna M, Tramontina J, Andreazza AC, Cereser K, da Costa S, Santin A, Yatham LN, and Kapczinski F (2007) Traumatic life events in bipolar disorder: impact on BDNF levels and psychopathology. Bipolar Disord 9 (Suppl 1):128-135. Kaufman J (2018) Unraveling the genetics of major depression and stress-related psychiatric disorders: is it time for a paradigm shift?. Biol Psychiatry 84:82-84.

Kay DC and Martin WR (1978) LSD and tryptamine effects on sleep/wakefulness and electrocorticogram patterns in intact cats. Psychopharmacology (Berl) 58:223-228. Kegeles LS, Martinez D, Kochan LD, Hwang DR, Huang Y, Mawlawi O, Suckow RF, Van Heertum RL, and Laruelle M (2002) NMDA antagonist effects on striatal dopamine release: positron emission tomography studies in humans. Synapse $\mathbf{4 3}$ $19-29$. 
Kehne JH, Baron BM, Carr AA, Chaney SF, Elands J, Feldman DJ, Frank RA, van Giersbergen PL, McCloskey TC, Johnson MP, et al. (1996) Preclinical characterization of the potential of the putative atypical antipsychotic MDL 100,907 as a potent 5-HT2A antagonist with a favorable CNS safety profile. J Pharmacol Exp Ther 277:968-981.

Keiser MJ, Setola V, Irwin JJ, Laggner C, Abbas AI, Hufeisen SJ, Jensen NH, Kuijer MB, Matos RC, Tran TB, et al. (2009) Predicting new molecular targets for known drugs. Nature 462:175-181.

Keller J, Gomez R, Williams G, Lembke A, Lazzeroni L, Murphy GM Jr, and Schatzberg AF (2017) HPA axis in major depression: cortisol, clinical symptomatology and genetic variation predict cognition. Mol Psychiatry 22:527-536.

Kenakin T (2015) The effective application of biased signaling to new drug discovery. Mol Pharmacol 88:1055-1061.

Kenakin T (2019) Biased receptor signaling in drug discovery. Pharmacol Rev $\mathbf{7 1}$ $267-315$

Kenakin T and Christopoulos A (2013) Signalling bias in new drug discovery: detection, quantification and therapeutic impact. Nat Rev Drug Discov 12:205-216.

Kenis G and Maes M (2002) Effects of antidepressants on the production of cytokines. Int J Neuropsychopharmacol 5:401-412.

Kessler RC, Petukhova M, Sampson NA, Zaslavsky AM, and Wittchen HU (2012) Twelve-month and lifetime prevalence and lifetime morbid risk of anxiety and mood disorders in the United States. Int J Methods Psychiatr Res 21:169-184.

Kikuchi-Utsumi K and Nakaki T (2008) Chronic treatment with a selective ligand for the sigma-1 receptor chaperone, SA4503, up-regulates BDNF protein levels in the rat hippocampus. Neurosci Lett 440:19-22.

Kim FJ and Pasternak GW (2018) $\sigma_{1}$ Receptor ligand binding: an open-and-shut case. Nat Struct Mol Biol 25:992-993.

Kim J, Farchione T, Potter A, Chen Q, and Temple R (2019) Esketamine for treatment-resistant depression - first FDA-approved antidepressant in a new class. $N$ Engl J Med 381:1-4.

Kim YK, Lee HP, Won SD, Park EY, Lee HY, Lee BH, Lee SW, Yoon D, Han C, Kim DJ, et al. (2007) Low plasma BDNF is associated with suicidal behavior in major depression. Prog Neuropsychopharmacol Biol Psychiatry 31:78-85.

Kindlundh-Högberg AM, Blomqvist A, Malki R, and Schiöth HB (2008) Extensive neuroadaptive changes in cortical gene-transcript expressions of the glutamate system in response to repeated intermittent MDMA administration in adolescent rats. BMC Neurosci 9:39.

Kinzie E and Meltzer-Brody S (2005) Possible serotonin syndrome with citalopram following cross-titration of clozapine to ziprasidone. Gen Hosp Psychiatry 27: $223-224$.

Kiraly DD, Horn SR, Van Dam NT, Costi S, Schwartz J, Kim-Schulze S, Patel M, Hodes GE, Russo SJ, Merad M, et al. (2017) Altered peripheral immune profiles in treatment-resistant depression: response to ketamine and prediction of treatment outcome. Transl Psychiatry 7:e1065.

Kirkpatrick MG, Francis SM, Lee R, de Wit H, and Jacob S (2014a) Plasma oxytocin concentrations following MDMA or intranasal oxytocin in humans. Psychoneuroendocrinology 46:23-31.

Kirkpatrick MG, Lee R, Wardle MC, Jacob S, and de Wit H (2014b) Effects of MDMA and Intranasal oxytocin on social and emotional processing. Neuropsychopharmacology 39:1654-1663.

Klimek V, Stockmeier C, Overholser J, Meltzer HY, Kalka S, Dilley G, and Ordway GA (1997) Reduced levels of norepinephrine transporters in the locus coeruleus in major depression. J Neurosci 17:8451-8458.

Knuijver T, Belgers M, Markus W, Verkes RJ, van Oosteren T, and Schellekens A (2018) Hallucinogen persisting perception disorder after ibogaine treatment for opioid dependence. J Clin Psychopharmacol 38:646-648.

Koch S and Galloway MP (1997) MDMA induced dopamine release in vivo: role of endogenous serotonin. J Neural Transm (Vienna) 104:135-146.

Koch SB, van Zuiden M, Nawijn L, Frijling JL, Veltman DJ, and Olff M (2014) Intranasal oxytocin as strategy for medication-enhanced psychotherapy of PTSD: salience processing and fear inhibition processes. Psychoneuroendocrinology 40:242-256.

Koehntop DE, Liao JC, and Van Bergen FH (1977) Effects of pharmacologic alterations of adrenergic mechanisms by cocaine, tropolone, aminophylline, and ketamine on epinephrine-induced arrhythmias during halothane-nitrous oxide anesthesia. Anesthesiology 46:83-93.

Koenen KC, Amstadter AB, and Nugent NR (2009) Gene-environment interaction in posttraumatic stress disorder: an update. J Trauma Stress 22:416-426.

Köhler O, Benros ME, Nordentoft M, Farkouh ME, Iyengar RL, Mors O, and Krogh J (2014) Effect of anti-inflammatory treatment on depression, depressive symptoms, and adverse effects: a systematic review and meta-analysis of randomized clinical trials. JAMA Psychiatry 71:1381-1391.

Köhler-Forsberg O, N Lydholm C, Hjorthøj C, Nordentoft M, Mors O, and Benros ME (2019) Efficacy of anti-inflammatory treatment on major depressive disorder or depressive symptoms: meta-analysis of clinical trials. Acta Psychiatr Scand 139: 404-419.

Kometer M, Schmidt A, Bachmann R, Studerus E, Seifritz E, and Vollenweider FX (2012) Psilocybin biases facial recognition, goal-directed behavior, and mood state toward positive relative to negative emotions through different serotonergic subreceptors. Biol Psychiatry 72:898-906.

Kometer M, Schmidt A, Jäncke L, and Vollenweider FX (2013) Activation of serotonin $2 \mathrm{~A}$ receptors underlies the psilocybin-induced effects on $\alpha$ oscillations, N170 visualevoked potentials, and visual hallucinations. J Neurosci 33:10544-10551.

Koslow SH, Maas JW, Bowden CL, Davis JM, Hanin I, and Javaid J (1983) CSF and urinary biogenic amines and metabolites in depression and mania. A controlled, univariate analysis. Arch Gen Psychiatry 40:999-1010.

Kosten TR, Mason JW, Giller EL, Ostroff RB, and Harkness L (1987) Sustained urinary norepinephrine and epinephrine elevation in post-traumatic stress disorder. Psychoneuroendocrinology 12:13-20.

Kotas ME and Medzhitov R (2015) Homeostasis, inflammation, and disease susceptibility. Cell 160:816-827.
Kourrich S (2017) Sigma-1 receptor and neuronal excitability, in Sigma Proteins: Evolution of the Concept of Sigma Receptors (Kim FJ and Pasternak GW eds) pp 109-130, Springer International Publishing, Cham, Switzerland.

Kourrich S, Hayashi T, Chuang JY, Tsai SY, Su TP, and Bonci A (2013) Dynamic interaction between sigma-1 receptor and Kv1.2 shapes neuronal and behavioral responses to cocaine. Cell 152:236-247.

Kourrich S, Su T-P, Fujimoto M, and Bonci A (2012) The sigma-1 receptor: roles in neuronal plasticity and disease. Trends Neurosci 35:762-771.

Kozlovsky N, Matar MA, Kaplan Z, Kotler M, Zohar J, and Cohen H (2007) Long-term down-regulation of BDNF mRNA in rat hippocampal CA1 subregion correlates with PTSD-like behavioural stress response. Int J Neuropsychopharmacol 10:741-758.

Kraehenmann R (2017) Dreams and psychedelics: neurophenomenological comparison and therapeutic implications. Curr Neuropharmacol 15:1032-1042.

Kraehenmann R, Pokorny D, Vollenweider L, Preller KH, Pokorny T, Seifritz E, and Vollenweider FX (2017) Dreamlike effects of LSD on waking imagery in humans depend on serotonin $2 \mathrm{~A}$ receptor activation. Psychopharmacology (Berl) 234:2031-2046.

Krall CM, Richards JB, Rabin RA, and Winter JC (2008) Marked decrease of LSDinduced stimulus control in serotonin transporter knockout mice. Pharmacol Biochem Behav 88:349-357.

Kraus C, Castrén E, Kasper S, and Lanzenberger R (2017) Serotonin and neuroplasticity - links between molecular, functional and structural pathophysiology in depression. Neurosci Biobehav Rev 77:317-326.

Krebs TS and Johansen PO (2013) Psychedelics and mental health: a population study. PLoS One 8:1-9.

Krebs-Thomson K, Ruiz EM, Masten V, Buell M, and Geyer MA (2006) The roles of 5HT1A and 5-HT2 receptors in the effects of 5-MeO-DMT on locomotor activity and prepulse inhibition in rats. Psychopharmacology (Berl) 189:319-329.

Kreibig SD (2010) Autonomic nervous system activity in emotion: a review. Biol Psychol 84:394-421.

Krimmel SR, White MG, Panicker MH, Barrett FS, Mathur BN, and Seminowicz DA (2019) Resting state functional connectivity and cognitive task-related activation of the human claustrum. Neuroimage 196:59-67.

Kringelbach ML, Cruzat J, Cabral J, Knudsen GM, Carhart-Harris R, Whybrow PC Logothetis NK, and Deco G (2020) Dynamic coupling of whole-brain neuronal and neurotransmitter systems. Proc Natl Acad Sci USA 117:9566-9576.

Kroes MC, Rugg MD, Whalley MG, and Brewin CR (2011) Structural brain abnormalities common to posttraumatic stress disorder and depression. J Psychiatry Neurosci 36:256-265.

Kroeze WK and Roth BL (1998) The molecular biology of serotonin receptors: therapeutic implications for the interface of mood and psychosis. Biol Psychiatry 44: $1128-1142$.

Krsiak M, Borgesovã M, and Kadlecovã O (1971) LSD-accentuated individual type of social behavior in mice. Act Nerv Super (Praha) 13:211-212.

Krystal JH, Abdallah CG, Sanacora G, Charney DS, and Duman RS (2019) Ketamine: a paradigm shift for depression research and treatment. Neuron 101: $774-778$

Kubota T, Hirota K, Anzawa N, Yoshida H, Kushikata T, and Matsuki A (1999) Physostigmine antagonizes ketamine-induced noradrenaline release from the medial prefrontal cortex in rats. Brain Res 840:175-178.

Kuipers SD, Trentani A, Den Boer JA, and Ter Horst GJ (2003) Molecular correlates of impaired prefrontal plasticity in response to chronic stress. J Neurochem $\mathbf{8 5}$ $1312-1323$.

Kular L and Kular S (2018) Epigenetics applied to psychiatry: clinical opportunities and future challenges. Psychiatry Clin Neurosci 72:195-211.

Kurtom M, Henning A, and Espiridion ED (2019) Hallucinogen-persisting perception disorder in a 21-year-old man. Cureus 11:e4077.

Kuypers KP, Ng L, Erritzoe D, Knudsen GM, Nichols CD, Nichols DE, Pani L, Soula A, and Nutt D (2019) Microdosing psychedelics: more questions than answers? An overview and suggestions for future research. J Psychopharmacol 33:1039-1057.

Kuypers KP, Riba J, de la Fuente Revenga M, Barker S, Theunissen EL and Ramaekers JG (2016) Ayahuasca enhances creative divergent thinking while decreasing conventional convergent thinking. Psychopharmacology (Berl) 233: 3395-3403.

Kyzar EJ, Stewart AM, and Kalueff AV (2016) Effects of LSD on grooming behavior in serotonin transporter heterozygous ( $\mathrm{Sert}^{+} /^{-}$) mice. Behav Brain Res 296:47-52

Labate BC and Feeney K (2012) Ayahuasca and the process of regulation in Brazil and internationally: implications and challenges. Int J Drug Policy 23:154-161.

Lahoud N and Maroun M (2013) Oxytocinergic manipulations in corticolimbic circuit differentially affect fear acquisition and extinction. Psychoneuroendocrinology 38: $2184-2195$

Lambe EK and Aghajanian GK (2006) Hallucinogen-induced UP states in the brain slice of rat prefrontal cortex: role of glutamate spillover and NR2B-NMDA receptors. Neuropsychopharmacology 31:1682-1689.

Landmann N, Kuhn M, Maier JG, Spiegelhalder K, Baglioni C, Frase L, Riemann D, Sterr A, and Nissen C (2015) REM sleep and memory reorganization: potential relevance for psychiatry and psychotherapy. Neurobiol Learn Mem 122:28-40.

Langer SZ, Lee CR, Segonzac A, Tateishi T, Esnaud H, Schoemaker H, and Winblad B (1984) Possible endocrine role of the pineal gland for 6-methoxytetrahydro-betacarboline, a putative endogenous neuromodulator of the $[3 \mathrm{H}]$ imipramine recognition site. Eur J Pharmacol 102:379-380.

Lapidus KA, Levitch CF, Perez AM, Brallier JW, Parides MK, Soleimani L, Feder A, Iosifescu DV, Charney DS, and Murrough JW (2014) A randomized controlled trial of intranasal ketamine in major depressive disorder. Biol Psychiatry 76:970-976.

Layer RT, Popik P, Olds T, and Skolnick P (1995) Antidepressant-like actions of the polyamine site NMDA antagonist, eliprodil (SL-82.0715). Pharmacol Biochem Behav 52:621-627.

Lea T, Amada N, Jungaberle H, Schecke H, Scherbaum N, and Klein M (2020) Perceived outcomes of psychedelic microdosing as self-managed therapies for mental and substance use disorders. Psychopharmacology (Berl) 237:1521-1532. 
Lebedev AV, Kaelen M, Lövdén M, Nilsson J, Feilding A, Nutt DJ, and CarhartHarris RL (2016) LSD-induced entropic brain activity predicts subsequent personality change. Hum Brain Mapp 37:3203-3213.

Lebedev AV, Lövdén M, Rosenthal G, Feilding A, Nutt DJ, and Carhart-Harris RL (2015) Finding the self by losing the self: neural correlates of ego-dissolution under psilocybin. Hum Brain Mapp 36:3137-3153.

Lee BH, Kim H, Park SH, and Kim YK (2007) Decreased plasma BDNF level in depressive patients. J Affect Disord 101:239-244.

Lee HJ, Lee MS, Kang RH, Kim H, Kim SD, Kee BS, Kim YH, Kim YK, Kim JB, Yeon $\mathrm{BK}$, et al. (2005) Influence of the serotonin transporter promoter gene polymorphism on susceptibility to posttraumatic stress disorder. Depress Anxiety 21: $135-139$.

Leighton SP, Nerurkar L, Krishnadas R, Johnman C, Graham GJ, and Cavanagh J (2018) Chemokines in depression in health and in inflammatory illness: a systematic review and meta-analysis. Mol Psychiatry 23:48-58.

Leino M (1984) 6-Methoxy-tetrahydro-beta-carboline and melatonin in the human retina. Exp Eye Res 38:325-330.

Leonard BE and Tonge SR (1969) The effects of some hallucinogenic drugs upon the metabolism of noradrenaline. Life Sci 8:815-825.

Leonard HL and Rapoport JL (1987) Relief of obsessive-compulsive symptoms by LSD and psilocin. American J Psychiatry 144:1239-1240.

Leonardi ET and Azmitia EC (1994) MDMA (ecstasy) inhibition of MAO type A and type B: comparisons with fenfluramine and fluoxetine (Prozac). Neuropsychopharmacology 10:231-238.

Lerner AG, Gelkopf M, Skladman I, Rudinski D, Nachshon H, and Bleich A (2003) Clonazepam treatment of lysergic acid diethylamide-induced hallucinogen persisting perception disorder with anxiety features. Int Clin Psychopharmacol 18 $101-105$.

Lesch KP, Bengel D, Heils A, Sabol SZ, Greenberg BD, Petri S, Benjamin J, Müller CR, Hamer DH, and Murphy DL (1996) Association of anxiety-related traits with a polymorphism in the serotonin transporter gene regulatory region. Science $\mathbf{2 7 4}$ 1527-1531

Leung LY and Baillie TA (1989) Studies on the biotransformation of ketamine. IIQuantitative significance of the N-demethylation pathway in rats in vivo de termined by a novel stable isotope technique. Biomed Environ Mass Spectrom 18 401-404.

Levänen J, Mäkelä ML, and Scheinin H (1995) Dexmedetomidine premedication attenuates ketamine-induced cardiostimulatory effects and postanesthetic delirium. Anesthesiology 82:1117-1125.

Lewis CR, Preller KH, Braden BB, Riecken C, and Vollenweider FX (2020) Rostral anterior cingulate thickness predicts the emotional psilocybin experience. Biomedicines 8:34.

Lewis CR, Preller KH, Kraehenmann R, Michels L, Staempfli P, and Vollenweider FX (2017) Two dose investigation of the 5-HT-agonist psilocybin on relative and global cerebral blood flow. Neuroimage 159:70-78

Li L, Carter J, Gao X, Whitehead J, and Tourtellotte WG (2005) The neuroplasticityassociated arc gene is a direct transcriptional target of early growth response (Egr) transcription factors. Mol Cell Biol 25:10286-10300.

Li N, Lee B, Liu RJ, Banasr M, Dwyer JM, Iwata M, Li XY, Aghajanian G, and Duman RS (2010) mTOR-dependent synapse formation underlies the rapid antidepressant effects of NMDA antagonists. Science 329:959-964.

Li W, Mai X, and Liu C (2014) The default mode network and social understanding of others: what do brain connectivity studies tell us. Front Hum Neurosci 8:74.

Li Y, Shen R, Wen G, Ding R, Du A, Zhou J, Dong Z, Ren X, Yao H, Zhao R, et al. (2017) Effects of ketamine on levels of inflammatory cytokines IL-6, IL-1 $\beta$, and TNF- $\alpha$ in the hippocampus of mice following acute or chronic administration. Front Pharmacol 8:139.

Lieberman JA, Mailman RB, Duncan G, Sikich L, Chakos M, Nichols DE, and Kraus JE (1998) Serotonergic basis of antipsychotic drug effects in schizophrenia. Biol Psychiatry 44:1099-1117.

Liechti ME, Baumann C, Gamma A, and Vollenweider FX (2000) Acute psychological effects of 3,4-methylenedioxymethamphetamine (MDMA, "Ecstasy") are attenuated by the serotonin uptake inhibitor citalopram. Neuropsychopharmacology 22: $513-521$

Liechti ME, Dolder PC, and Schmid Y (2017) Alterations of consciousness and mystical-type experiences after acute LSD in humans. Psychopharmacology (Berl) 234:1499-1510

Liechti ME, Gamma A, and Vollenweider FX (2001) Gender differences in the subjective effects of MDMA. Psychopharmacology (Berl) 154:161-168.

Liester MB and Prickett JI (2012) Hypotheses regarding the mechanisms of ayahuasca in the treatment of addictions. J Psychoactive Drugs 44:200-208.

Lima da Cruz RV, Moulin TC, Petiz LL, and Leão RN (2018) A single dose of 5-MeODMT stimulates cell proliferation, neuronal survivability, morphological and functional changes in adult mice ventral dentate gyrus. Front Mol Neurosci 11:312

Lindefors N, Barati S, and O'Connor WT (1997) Differential effects of single and repeated ketamine administration on dopamine, serotonin and GABA transmission in rat medial prefrontal cortex. Brain Res 759:205-212.

Lindqvist D, Dhabhar FS, Mellon SH, Yehuda R, Grenon SM, Flory JD, Bierer LM, Abu-Amara D, Coy M, Makotkine I, et al. (2017) Increased pro-inflammatory milieu in combat related PTSD - a new cohort replication study. Brain Behav Immun 59:260-264.

Lisek M, Ferenc B, Studzian M, Pulaski L, Guo F, Zylinska L, and Boczek T (2017) Glutamate deregulation in ketamine-induced psychosis-A potential role of PSD95, NMDA receptor and PMCA interaction. Front Cell Neurosci 11:181.

Liu Y, Lin D, Wu B, and Zhou W (2016) Ketamine abuse potential and use disorder. Brain Res Bull 126:68-73.

Lloyd AF, Davies CL, Holloway RK, Labrak Y, Ireland G, Carradori D, Dillenburg A Borger E, Soong D, Richardson JC, et al. (2019) Central nervous system regeneration is driven by microglia necroptosis and repopulation. Nat Neurosci 22 : $1046-1052$
Lloyd KG, Thuret F, and Pilc A (1985) Upregulation of gamma-aminobutyric acid (GABA) B binding sites in rat frontal cortex: a common action of repeated administration of different classes of antidepressants and electroshock. $J$ Pharmacol Exp Ther 235:191-199.

Lodge D, Anis NA, and Burton NR (1982) Effects of optical isomers of ketamine on excitation of cat and rat spinal neurones by amino acids and acetylcholine. Neurosci Lett 29:281-286.

López-Gil X, Jiménez-Sánchez L, Campa L, Castro E, Frago C, and Adell A (2019) Role of serotonin and noradrenaline in the rapid antidepressant action of ketamine. ACS Chem Neurosci 10:3318-3326.

Lopez-Gimenez JF, Canals M, Pediani JD, and Milligan G (2007) The alpha1badrenoceptor exists as a higher-order oligomer: effective oligomerization is required for receptor maturation, surface delivery, and function. Mol Pharmacol 71: $1015-1029$.

Lord CC, Wyler SC, Wan R, Castorena CM, Ahmed N, Mathew D, Lee S, Liu C, and Elmquist JK (2017) The atypical antipsychotic olanzapine causes weight gain by targeting serotonin receptor 2C. J Clin Invest 127:3402-3406.

Lotrich FE (2009) Major depression during interferon-alpha treatment: vulnerability and prevention. Dialogues Clin Neurosci 11:417-425.

Louveau A, Smirnov I, Keyes TJ, Eccles JD, Rouhani S.J, Peske JD, Derecki NC, Castle D, Mandell JW, Lee KS, et al. (2015) Structural and functional features of central nervous system lymphatic vessels. Nature 523:337-341.

Lovenberg TW, Erlander MG, Baron BM, Racke M, Slone AL, Siegel BW, Craft CM, Burns JE, Danielson PE, and Sutcliffe JG (1993) Molecular cloning and functional expression of 5-HT1E-like rat and human 5-hydroxytryptamine receptor genes. Proc Natl Acad Sci USA 90:2184-2188

$\mathrm{Lu} \mathrm{J,} \mathrm{Xu} \mathrm{Y,} \mathrm{Hu} \mathrm{W,} \mathrm{Gao} \mathrm{Y,} \mathrm{Ni} \mathrm{X,} \mathrm{Sheng} \mathrm{H,} \mathrm{and} \mathrm{Liu} \mathrm{Y} \mathrm{(2014)} \mathrm{Exercise} \mathrm{ameliorates}$ depression-like behavior and increases hippocampal BDNF level in ovariectomized rats. Neurosci Lett 573:13-18.

Łukasiewicz S, Błasiak E, Szafran-Pilch K, and Dziedzicka-Wasylewska M (2016) Dopamine D2 and serotonin 5-HT1A receptor interaction in the context of the effects of antipsychotics - in vitro studies. J Neurochem 137:549-560.

Lukasiewicz S, Polit A, Kędracka-Krok S, Wędzony K, Maćkowiak M, and Dziedzicka-Wasylewska M (2010) Hetero-dimerization of serotonin 5-HT(2A) and dopamine $\mathrm{D}(2)$ receptors. Biochim Biophys Acta 1803:1347-1358.

Lur G, Fariborzi M, and Higley MJ (2019) Ketamine disrupts neuromodulatory control of glutamatergic synaptic transmission. PLoS One 14:e0213721.

Luttrell LM, Ferguson SS, Daaka Y, Miller WE, Maudsley S, Della Rocca GJ, Lin F, Kawakatsu H, Owada K, Luttrell DK, et al. (1999) Beta-arrestin-dependent formation of beta2 adrenergic receptor-Src protein kinase complexes. Science $\mathbf{2 8 3}$ : 655-661

Ly C, Greb AC, Cameron LP, Wong JM, Barragan EV, Wilson PC, Burbach KF, Soltanzadeh Zarandi S, Sood A, Paddy MR, et al (2018) Psychedelics promote structural and functional neural plasticity. Cell Rep 23:3170-3182.

Lyons T and Carhart-Harris RL (2018) Increased nature relatedness and decreased authoritarian political views after psilocybin for treatment-resistant depression. J Psychopharmacol 32:811-819.

MacDonald JF, Miljkovic Z, and Pennefather P (1987) Use-dependent block of excitatory amino acid currents in cultured neurons by ketamine. $J$ Neurophysiol $\mathbf{5 8}$ : 251-266

MacDonald K, MacDonald TM, Brüne M, Lamb K, Wilson MP, Golshan S, and Feifel D (2013) Oxytocin and psychotherapy: a pilot study of its physiological, behaviora and subjective effects in males with depression. Psychoneuroendocrinology 38: 2831-2843

Maciulaitis R, Kontrimaviciute V, Bressolle FM, and Briedis V (2008) Ibogaine, an anti-addictive drug: pharmacology and time to go further in development. A narrative review. Hum Exp Toxicol 27:181-194.

MacQueen GM, Campbell S, McEwen BS, Macdonald K, Amano S, Joffe RT, Nahmias C, and Young LT (2003) Course of illness, hippocampal function, and hippocampal volume in major depression. Proc Natl Acad Sci USA 100:1387-1392.

Madsen MK, Fisher PM, Burmester D, Dyssegaard A, Stenbæk DS, Kristiansen S, Johansen SS, Lehel S, Linnet K, Svarer C, et al. (2019) Psychedelic effects of psilocybin correlate with serotonin $2 \mathrm{~A}$ receptor occupancy and plasma psilocin levels. Neuropsychopharmacology 44:1328-1334.

Maeng S, Zarate CA Jr, Du J, Schloesser RJ, McCammon J, Chen G, and Manji HK (2008) Cellular mechanisms underlying the antidepressant effects of ketamine: role of alpha-amino-3-hydroxy-5-methylisoxazole-4-propionic acid receptors. Biol Psychiatry 63:349-352.

Mahdavi A, Qin Y, Aubry AS, Cornec D, Kulikova S, and Pinault D (2020) A single psychotomimetic dose of ketamine decreases thalamocortical spindles and delta oscillations in the sedated rat. Schizophr Res DOI: 10.1016/j.schres.2020.04.029 [published ahead of print].

Mahmoudi E, Faizi M, Hajiaghaee R, and Razmi A (2018) Alteration of depressivelike behaviors by Psilocybe cubensis alkaloid extract in mice: the role of glutamate pathway. Res J Pharmacogn 5:17-24.

Maier SF and Watkins LR (2003) Immune-to-central nervous system communication and its role in modulating pain and cognition: implications for cancer and cancer treatment. Brain Behav Immun 17 (Suppl 1):S125-S131.

Malberg JE, Eisch AJ, Nestler EJ, and Duman RS (2000) Chronic antidepressant treatment increases neurogenesis in adult rat hippocampus. $J$ Neurosci 20: 9104-9110

Maldonado-López R and Moser M (2001) Dendritic cell subsets and the regulation of Th1/Th2 responses. Semin Immunol 13:275-282.

Malitz S, Wilkens B, Roehrig WC, and Hoch PH (1960) A clinical comparison of three related hallucinogens. Psychiatr Q 34:333-345.

Mandela P and Ordway GA (2006) The norepinephrine transporter and its regulation. J Neurochem 97:310-333.

Manglik A, Lin H, Aryal DK, McCorvy JD, Dengler D, Corder G, Levit A, Kling RC, Bernat V, Hübner H, et al. (2016) Structure-based discovery of opioid analgesics with reduced side effects. Nature 537:185-190. 
Mantz J, Lecharny JB, Laudenbach V, Henzel D, Peytavin G, and Desmonts JM (1995) Anesthetics affect the uptake but not the depolarization-evoked release of GABA in rat striatal synaptosomes. Anesthesiology 82:502-511.

Marek GJ (2018) Interactions of hallucinogens with the glutamatergic system: permissive network effects mediated through cortical layer V pyramidal neurons. Curr Top Behav Neurosci 36:107-135.

Marek GJ and Aghajanian GK (1994) Excitation of interneurons in piriform cortex by 5-hydroxytryptamine: blockade by MDL 100,907, a highly selective 5-HT2A receptor antagonist. Eur J Pharmacol 259:137-141.

Marek GJ and Aghajanian GK (1996) LSD and the phenethylamine hallucinogen DOI are potent partial agonists at 5-HT2A receptors on interneurons in rat piriform cortex. J Pharmacol Exp Ther 278:1373-1382.

Marek GJ, Wright RA, Gewirtz JC, and Schoepp DD (2001) A major role for thalamocortical afferents in serotonergic hallucinogen receptor function in the rat neocortex. Neuroscience 105:379-392.

Marona-Lewicka D, Chemel BR, and Nichols DE (2009) Dopamine D4 receptor involvement in the discriminative stimulus effects in rats of LSD, but not the phenethylamine hallucinogen DOI. Psychopharmacology (Berl) 203:265-277.

Marona-Lewicka D, Nichols CD, and Nichols DE (2011) An animal model of schizophrenia based on chronic LSD administration: old idea, new results. Neuropharmacology 61:503-512.

Marona-Lewicka D and Nichols DE (1995) Complex stimulus properties of LSD: a drug discrimination study with alpha 2-adrenoceptor agonists and antagonists. Psychopharmacology (Berl) 120:384-391.

Marona-Lewicka D, Thisted RA, and Nichols DE (2005) Distinct temporal phases in the behavioral pharmacology of LSD: dopamine D2 receptor-mediated effects in the rat and implications for psychosis. Psychopharmacology (Berl) 180:427-435.

Marsden WN (2011) Stressor-induced NMDAR dysfunction as a unifying hypothesis for the aetiology, pathogenesis and comorbidity of clinical depression. Med Hypotheses 77:508-528.

Martín-Ruiz R, Puig MV, Celada P, Shapiro DA, Roth BL, Mengod G, and Artigas F (2001) Control of serotonergic function in medial prefrontal cortex by serotonin-2A receptors through a glutamate-dependent mechanism. J Neurosci 21:9856-9866.

Martin DA, Marona-Lewicka D, Nichols DE, and Nichols CD (2014) Chronic LSD alters gene expression profiles in the mPFC relevant to schizophrenia. Neuropharmacology 83:1-8.

Martin DA and Nichols CD (2016) Psychedelics recruit multiple cellular types and produce complex transcriptional responses within the brain. EBioMedicine 11: $262-277$

Martin LL, Bouchal RL, and Smith DJ (1982) Ketamine inhibits serotonin uptake in vivo. Neuropharmacology 21:113-118.

Martin P, Pichat P, Massol J, Soubrié P, Lloyd KG, and Puech AJ (1989) Decreased GABA B receptors in helpless rats: reversal by tricyclic antidepressants. Neuropsychobiology 22:220-224.

Martin TG (1996) Serotonin syndrome. Ann Emerg Med 28:520-526.

Martinotti G, Santacroce R, Pettorruso M, Montemitro C, Spano MC, Lorusso M, di Giannantonio M, and Lerner AG (2018) Hallucinogen persisting perception disorder: etiology, clinical features, and therapeutic perspectives. Brain Sci 8:47.

Martinowich K and Lu B (2008) Interaction between BDNF and serotonin: role in mood disorders. Neuropsychopharmacology 33:73-83.

Martinowich K, Manji H, and Lu B (2007) New insights into BDNF function in depression and anxiety. Nat Neurosci 10:1089-1093.

Mash DC and Zabetian CP (1992) Sigma receptors are associated with cortical limbic areas in the primate brain. Synapse 12:195-205.

Mason JW, Billingham ME, and Friedman JP (1977) Methysergide-induced heart disease: a case of multivalvular and myocardial fibrosis. Circulation 56:889-890.

Mastronardi C, Whelan F, Yildiz OA, Hannestad J, Elashoff D, McCann SM, Licinio J, and Wong ML (2007) Caspase 1 deficiency reduces inflammation-induced brain transcription. Proc Natl Acad Sci USA 104:7205-7210.

Mastronardi CA, Paz-Filho G, Zanoni M, Molano-González N, Arcos-Burgos M, Licinio J, and Wong ML (2015) Temporal gene expression in the hippocampus and peripheral organs to endotoxin-induced systemic inflammatory response in caspase-1-deficient mice. Neuroimmunomodulation 22:263-273.

Mathew SJ, Murrough JW, aan het Rot M, Collins KA, Reich DL, and Charney DS (2010) Riluzole for relapse prevention following intravenous ketamine in treatment-resistant depression: a pilot randomized, placebo-controlled continuation trial. Int $J$ Neuropsychopharmacol 13:71-82.

Maurice T and Su T-P (2009) The pharmacology of sigma-1 receptors. Pharmacol Ther 124:195-206.

Maurice T, Su TP, and Privat A (1998) Sigma1 (sigma 1) receptor agonists and neurosteroids attenuate B25-35-amyloid peptide-induced amnesia in mice through a common mechanism. Neuroscience 83:413-428.

Mayerhofer A, Kovar KA, and Schmidt WJ (2001) Changes in serotonin, dopamine and noradrenaline levels in striatum and nucleus accumbens after repeated administration of the abused drug MDMA in rats. Neurosci Lett 308:99-102

McAlonan K, Brown VJ, and Bowman EM (2000) Thalamic reticular nucleus activation reflects attentional gating during classical conditioning. $J$ Neurosci 20 8897-8901.

McAlonan K, Cavanaugh J, and Wurtz RH (2006) Attentional modulation of thalamic reticular neurons. J Neurosci 26:4444-4450.

McCall RB and Aghajanian GK (1980) Hallucinogens potentiate responses to serotonin and norepinephrine in the facial motor nucleus. Life Sci 26:1149-1156.

McCreary AC, Bankson MG, and Cunningham KA (1999) Pharmacological studies of the acute and chronic effects of (+)-3, 4-methylenedioxymethamphetamine on locomotor activity: role of 5-hydroxytryptamine(1A) and 5-hydroxytryptamine(1B/ 1D) receptors. J Pharmacol Exp Ther 290:965-973.

McGrath JC and Olverman HJ (1978) Release of [3H-noradrenaline from the motor adrenergic nerves of the anococcygeus muscle by lysergic acid diethylamide, tyramine or nerve stimulation. $\mathrm{Br} J$ Pharmacol 64:615-622.
McKenna DJ, Repke DB, Lo L, and Peroutka SJ (1990) Differential interactions of indolealkylamines with 5-hydroxytryptamine receptor subtypes. Neuropharmacology 29:193-198.

McKenna DJ and Towers GH (1985) On the comparative ethnopharmacology of malpighiaceous and myristicaceous hallucinogens. J Psychoactive Drugs 17:35-39.

McKenna DJ, Towers GH, and Abbott F (1984) Monoamine oxidase inhibitors in South American hallucinogenic plants: tryptamine and beta-carboline constituents of ayahuasca. J Ethnopharmacol 10:195-223.

McLean TH, Parrish JC, Braden MR, Marona-Lewicka D, Gallardo-Godoy A, and Nichols DE (2006) 1-Aminomethylbenzocycloalkanes: conformationally restricted hallucinogenic phenethylamine analogues as functionally selective 5HT2A receptor agonists. $J$ Med Chem 49:5794-5803.

Mechan AO, Esteban B, O'Shea E, Elliott JM, Colado MI, and Green AR (2002) The pharmacology of the acute hyperthermic response that follows administration of 3,4-methylenedioxymethamphetamine (MDMA, 'ecstasy') to rats. $\mathrm{Br} J$ Pharmacol 135:170-180.

Mehta P, McAuley DF, Brown M, Sanchez E, Tattersall RS, and Manson JJ; HLH Across Speciality Collaboration, UK (2020) COVID-19: consider cytokine storm syndromes and immunosuppression. Lancet 395:1033-1034.

Melhem NM, Keilp JG, Porta G, Oquendo MA, Burke A, Stanley B, Cooper TB, Mann JJ, and Brent DA (2016) Blunted HPA axis activity in suicide attempters compared to those at high risk for suicidal behavior. Neuropsychopharmacology $\mathbf{4 1}$ : $1447-1456$

Meltzer HY (1999) The role of serotonin in antipsychotic drug action. Neuropsychopharmacology 21(2 Suppl):106S-115S.

Meltzer HY and Massey BW (2011) The role of serotonin receptors in the action of atypical antipsychotic drugs. Curr Opin Pharmacol 11:59-67.

Messaoud A, Mensi R, Douki W, Neffati F, Najjar MF, Gobbi G, Valtorta F, Gaha L, and Comai S (2019) Reduced peripheral availability of tryptophan and increased activation of the kynurenine pathway and cortisol correlate with major depression and suicide. World J Biol Psychiatry 20:703-711.

Meunier J and Maurice T (2004) Beneficial effects of the sigma1 receptor agonists igmesine and dehydroepiandrosterone against learning impairments in rats prenatally exposed to cocaine. Neurotoxicol Teratol 26:783-797.

Meyer JH, McMain S, Kennedy SH, Korman L, Brown GM, DaSilva JN, Wilson AA Blak T, Eynan-Harvey R, Goulding VS, et al. (2003) Dysfunctional attitudes and 5HT2 receptors during depression and self-harm. Am J Psychiatry 160:90-99.

Miletich DJ, Ivankovic AD, Albrecht RF, Zahed B, and Ilahi AA (1973) The effect of ketamine on catecholamine metabolism in the isolated perfused rat heart. Anesthesiology 39:271-277.

Millan MJ (2005) Serotonin 5-HT2C receptors as a target for the treatment of depressive and anxious states: focus on novel therapeutic strategies. Therapie 60: 441-460.

Miller KW and Gonzalez HA (1998) Serotonin 5?HT2A receptor activation inhibits cytokine?stimulated inducible nitric oxide synthase in C6 glioma cells. Annals of the New York Academy of Sciences 861:169-173.

Miller RT, Lau SS, and Monks TJ (1996) Effects of intracerebroventricular administration of 5-(glutathion-S-yl)- $\alpha$-methyldopamine on brain dopamine, serotonin, and norepinephrine concentrations in male Sprague-Dawley rats. Chem Res Toxicol 9:457-465.

Miller RT, Lau SS, and Monks TJ (1997) 2,5-Bis-(glutathion-S-yl)-alpha-methyldopamine, a putative metabolite of (+/-)-3,4-methylenedioxyamphetamine, decreases brain serotonin concentrations. Eur J Pharmacol 323:173-180.

Min BK (2010) A thalamic reticular networking model of consciousness. Theor Biol Med Model 7:10

Miralles A, Esteban S, Sastre-Coll A, Moranta D, Asensio VJ, and García-Sevilla JA (2005) High-affinity binding of beta-carbolines to imidazoline I2B receptors and MAO-A in rat tissues: norharman blocks the effect of morphine withdrawal on DOPA/noradrenaline synthesis in the brain. Eur J Pharmacol 518:234-242.

Mithoefer M, Jerome L, and Doblin R (2003) MDMA ("ecstasy") and neurotoxicity. Science 300:1504-1505, author reply 1504-1505.

Mithoefer MC, Feduccia AA, Jerome L, Mithoefer A, Wagner M, Walsh Z, Hamilton S, Yazar-Klosinski B, Emerson A, and Doblin R (2019) MDMA-assisted psychotherapy for treatment of PTSD: study design and rationale for phase 3 trials based on pooled analysis of six phase 2 randomized controlled trials. Psychopharmacology (Berl) 236:2735-2745

Mithoefer MC, Grob CS, and Brewerton TD (2016) Novel psychopharmacological therapies for psychiatric disorders: psilocybin and MDMA. Lancet Psychiatry 3 481-488.

Mithoefer MC, Mithoefer AT, Feduccia AA, Jerome L, Wagner M, Wymer J, Holland J, Hamilton S, Yazar-Klosinski B, Emerson A, et al. (2018) 3,4-methylenedioxymethamphetamine (MDMA)-assisted psychotherapy for post-traumatic stress disorder in military veterans, firefighters, and police officers: a randomised, double-blind, dose-response, phase 2 clinical trial. Lancet Psychiatry 5:486-497.

Mithoefer MC, Wagner MT, Mithoefer AT, Jerome L, and Doblin R (2011) The safety and efficacy of +/-3,4-methylenedioxymethamphetamine-assisted psychotherapy in subjects with chronic, treatment-resistant posttraumatic stress disorder: the first randomized controlled pilot study. J Psychopharmacol 25:439-452.

Mithoefer MC, Wagner MT, Mithoefer AT, Jerome L, Martin SF, Yazar-Klosinski B, Michel Y, Brewerton TD, and Doblin R (2013) Durability of improvement in posttraumatic stress disorder symptoms and absence of harmful effects or drug dependency after 3,4-methylenedioxymethamphetamine-assisted psychotherapy: a prospective long-term follow-up study. J Psychopharmacol 27:28-39.

Miyaoka T, Wake R, Furuya M, Liaury K, Ieda M, Kawakami K, Tsuchie K, Taki M, Ishihara K, Araki T, et al. (2012) Minocycline as adjunctive therapy for patients with unipolar psychotic depression: an open-label study. Prog Neuropsychopharmacol Biol Psychiatry 37:222-226.

Modabbernia A, Taslimi S, Brietzke E, and Ashrafi M (2013) Cytokine alterations in bipolar disorder: a meta-analysis of 30 studies. Biol Psychiatry 74:15-25. 
Modak T, Bhad R, and Rao R (2019) A rare case of physical dependence with psychedelic LSD - a case report. J Subst Use 24:347-349.

Moghaddam B, Adams B, Verma A, and Daly D (1997) Activation of glutamatergic neurotransmission by ketamine: a novel step in the pathway from NMDA receptor blockade to dopaminergic and cognitive disruptions associated with the prefrontal cortex. J Neurosci 17:2921-2927.

Moghaddam B and Javitt D (2011) From revolution to evolution: the glutamate hypothesis of schizophrenia and its implication for treatment. Neuropsychopharm $\mathbf{3 7}$ : $4-15$.

Molendijk ML, Spinhoven P, Polak M, Bus BA, Penninx BW, and Elzinga BM (2014) Serum BDNF concentrations as peripheral manifestations of depression: evidence from a systematic review and meta-analyses on 179 associations $(\mathrm{N}=9484)$. Mol Psychiatry 19:791-800.

Möller IR, Slivacka M, Nielsen AK, Rasmussen SGF, Gether U, Loland CJ, and Rand KD (2019) Conformational dynamics of the human serotonin transporter during substrate and drug binding. Nat Commun 10:1687.

Monks TJ, Bai F, Miller RT, and Lau SS (2001) Serotonergic neurotoxicity of methylenedioxyamphetamine and methylenedioxymetamphetamine, in Biological Reactive Intermediates VI: Chemical and Biological Mechanisms in Susceptibility to and Prevention of Environmental Diseases (Dansette PM, Snyder R, Delaforge M, Gibson GG, Greim H, Jollow DJ, Monks TJ, and Sipes IG eds) pp 397-406, Springer US, Boston.

Montoya AG, Sorrentino R, Lukas SE, and Price BH (2002) Long-term neuropsychiatric consequences of "ecstasy" (MDMA): a review. Harv Rev Psychiatry 10: 212-220.

Morales-García JA, de la Fuente Revenga M, Alonso-Gil S, Rodríguez-Franco MI, Feilding A, Perez-Castillo A, and Riba J (2017) The alkaloids of Banisteriopsis caapi, the plant source of the Amazonian hallucinogen Ayahuasca, stimulate adult neurogenesis in vitro. Sci Rep 7:5309.

Morales-Garcia JA, Calleja-Conde J, Lopez-Moreno JA, Alonso-Gil S, Sanz-SanCristobal M, Riba J, and Perez-Castillo A (2020) N,N-dimethyltryptamine compound found in the hallucinogenic tea ayahuasca, regulates adult neurogenesis in vitro and in vivo. Transl Psychiatry 10 (1).

Moreno FA, Wiegand CB, Taitano EK, and Delgado PL (2006) Safety, tolerability, and efficacy of psilocybin in 9 patients with obsessive-compulsive disorder. J Clin Psychiatry 67:1735-1740.

Moreno JL, Holloway T, Albizu L, Sealfon SC, and González-Maeso J (2011) Metabotropic glutamate mGlu2 receptor is necessary for the pharmacological and behavioral effects induced by hallucinogenic 5-HT2A receptor agonists. Neurosci Lett 493:76-79.

Moreno JL, Miranda-Azpiazu P, García-Bea A, Younkin J, Cui M, Kozlenkov A, BenEzra A, Voloudakis G, Fakira AK, Baki L, et al. (2016) Allosteric signaling through an mGlu2 and 5-HT2A heteromeric receptor complex and its potential contribution to schizophrenia. Sci Signal 9:ra5.

Moreno JL, Holloway T, Rayannavar V, Sealfon SC, and González-Maeso J (2013) Chronic treatment with LY341495 decreases 5-HT(2A) receptor binding and hallucinogenic effects of LSD in mice. Neurosci Lett 536:69-73.

Moriguchi S, Shinoda Y, Yamamoto Y, Sasaki Y, Miyajima K, Tagashira H, and Fukunaga K (2013) Stimulation of the sigma-1 receptor by DHEA enhances synaptic efficacy and neurogenesis in the hippocampal dentate gyrus of olfactory bulbectomized mice. PLoS One 8:e60863.

Mueller F, Lenz C, Dolder PC, Harder S, Schmid Y, Lang UE, Liechti ME, and Borgwardt S (2017) Acute effects of LSD on amygdala activity during processing of fearful stimuli in healthy subjects. Transl Psychiatry 7:e1084

Müller F, Brändle R, Liechti ME, and Borgwardt S (2019) Neuroimaging of chronic MDMA ("ecstasy") effects: a meta-analysis. Neurosci Biobehav Rev 96:10-20.

Müller F, Dolder PC, Schmidt A, Liechti ME, and Borgwardt S (2018) Altered network hub connectivity after acute LSD administration. Neuroimage Clin 18: 694-701.

Müller F, Lenz C, Dolder P, Lang U, Schmidt A, Liechti M, and Borgwardt S (2017) Increased thalamic resting-state connectivity as a core driver of LSD-induced hallucinations. Acta Psychiatr Scand 136:648-657.

Murat S, Bigot M, Chapron J, König GM, Kostenis E, Battaglia G, Nicoletti F, Bourinet E, Bockaert J, Marin P, et al. (2019) $5-\mathrm{HT}_{2 \mathrm{~A}}$ receptor-dependent phosphorylation of $\mathrm{mGlu}_{2}$ receptor at Serine 843 promotes $\mathrm{mGlu}_{2}$ receptor-operated $\mathrm{G}_{\mathrm{i} / \mathrm{o}}$ signaling. Mol Psychiatry 24:1610-1626.

Murphy-Beiner A and Soar K (2020) Ayahuasca's 'afterglow': improved mindfulness and cognitive flexibility in ayahuasca drinkers. Psychopharmacology (Berl) 237: 1161-1169.

Murrough JW, Abdallah CG, and Mathew SJ (2017) Targeting glutamate signalling in depression: progress and prospects. Nat Rev Drug Discov 16:472-486.

Murrough JW, Iosifescu DV, Chang LC, Al Jurdi RK, Green CE, Perez AM, Iqbal S, Pillemer S, Foulkes A, Shah A, et al. (2013a) Antidepressant efficacy of ketamine in treatment-resistant major depression: a two-site randomized controlled trial. Am J Psychiatry 170:1134-1142.

Murrough JW, Perez AM, Pillemer S, Stern J, Parides MK, aan het Rot M, Collins KA, Mathew SJ, Charney DS, and Iosifescu DV (2013b) Rapid and longer-term antidepressant effects of repeated ketamine infusions in treatment-resistant major depression. Biol Psychiatry 74:250-256.

Murrough JW, Soleimani L, DeWilde KE, Collins KA, Lapidus KA, Iacoviello BM, Lener M, Kautz M, Kim J, Stern JB, et al. (2015) Ketamine for rapid reduction of suicidal ideation: a randomized controlled trial. Psychol Med 45:3571-3580.

Muschamp JW, Regina MJ, Hull EM, Winter JC, and Rabin RA (2004) Lysergic acid diethylamide and [-]-2,5-dimethoxy-4-methylamphetamine increase extracellular glutamate in rat prefrontal cortex. Brain Res 1023:134-140.

Muthukumaraswamy SD, Carhart-Harris RL, Moran RJ, Brookes MJ, Williams TM, Errtizoe D, Sessa B, Papadopoulos A, Bolstridge M, Singh KD, et al. (2013) Broadband cortical desynchronization underlies the human psychedelic state. J Neurosci 33:15171-15183.

Muzio JN, Roffwarg HP, and Kaufman E (1966) Alterations in the nocturnal sleep cycle resulting from LSD. Electroencephalogr Clin Neurophysiol 21:313-324.
Myint AM, Leonard BE, Steinbusch HW, and Kim YK (2005) Th1, Th2, and Th3 cytokine alterations in major depression. J Affect Disord 88:167-173.

Nagai F, Nonaka R, and Satoh Hisashi Kamimura K (2007) The effects of nonmedically used psychoactive drugs on monoamine neurotransmission in rat brain. Eur J Pharmacol 559:132-137.

Nardou R, Lewis EM, Rothhaas R, Xu R, Yang A, Boyden E, and Dölen G (2019) Oxytocin-dependent reopening of a social reward learning critical period with MDMA. Nature 569:116-120

Nasca C, Zelli D, Bigio B, Piccinin S, Scaccianoce S, Nistico' R, and McEwen BS (2015) Stress dynamically regulates behavior and glutamatergic gene expression in hippocampus by opening a window of epigenetic plasticity. PNAS 112: 14690-14695.

Nash JF and Yamamoto BK (1992) Methamphetamine neurotoxicity and striatal glutamate release: comparison to 3,4-methylenedioxymethamphetamine. Brain Res 581:237-243.

Nau F Jr, Miller J, Saravia J, Ahlert T, Yu B, Happel KI, Cormier SA, and Nichols $\mathrm{CD}$ (2015) Serotonin $5-\mathrm{HT}_{2}$ receptor activation prevents allergic asthma in a mouse model. Am J Physiol Lung Cell Mol Physiol 308:L191-L198.

Nau F Jr, Yu B, Martin D, and Nichols CD (2013) Serotonin 5-HT2A receptor activation blocks TNF- $\alpha$ mediated inflammation in vivo. PLoS One 8:e75426.

Nedergaard OA (1973) Cocaine-like effect of ketamine on vascular adrenergic neurones. Eur J Pharmacol 23:153-161.

Nestler EJ, Peña CJ, Kundakovic M, Mitchell A, and Akbarian S (2016) Epigenetic basis of mental illness. Neuroscientist 22:447-463.

Netzband N, Ruffell S, Linton S, Tsang WF, and Wolff T (2020) Modulatory effects of ayahuasca on personality structure in a traditional framework. Psychopharmacology (Berl) 237:3161-3171.

Nguyen L, Lucke-Wold BP, Mookerjee SA, Cavendish JZ, Robson MJ, Scandinaro AL and Matsumoto RR (2015) Role of sigma-1 receptors in neurodegenerative diseases. J Pharmacol Sci 127:17-29.

Nguyen L, Robson MJ, Healy JR, Scandinaro AL, and Matsumoto RR (2014) Involvement of sigma-1 receptors in the antidepressant-like effects of dextromethorphan. PLoS One 9:e89985.

Nicholas CR, Henriquez KM, Gassman MC, Cooper KM, Muller D, Hetzel S, Brown RT, Cozzi NV, Thomas C, and Hutson PR (2018) High dose psilocybin is associated with positive subjective effects in healthy volunteers. J Psychopharmacol 32:770-778. Nichols CD and Sanders-Bush E (2004) Molecular genetic responses to lysergic acid diethylamide include transcriptional activation of MAP kinase phosphatase-1, C/EBP-beta and ILAD-1, a novel gene with homology to arrestins. J Neurochem 90: $576-584$

Nichols DE (1986) Differences between the mechanism of action of MDMA, MBDB, and the classic hallucinogens. Identification of a new therapeutic class: entactogens. J Psychoactive Drugs 18:305-313.

Nichols DE (2016) Psychedelics. Pharmacol Rev 68:264-355.

Nichols DE and Grob CS (2018) Is LSD toxic?. Forensic Sci Int 284:141-145.

Nichols DE and Nichols CD (2008) Serotonin receptors. Chem Rev 108:1614-1641.

Nielson EM, May DG, Forcehimes AA, and Bogenschutz MP (2018) The psychedelic debriefing in alcohol dependence treatment: illustrating key change phenomena through qualitative content analysis of clinical sessions. Front Pharmacol 9:132.

Nishimura M, Sato K, Okada T, Yoshiya I, Schloss P, Shimada S, and Tohyama M (1998) Ketamine inhibits monoamine transporters expressed in human embryonic kidney 293 cells. Anesthesiology 88:768-774.

Norimoto H, Fenk LA, Li HH, Tosches MA, Gallego-Flores T, Hain D, Reiter S, Kobayashi R, Macias A, Arends A, et al. (2020) A claustrum in reptiles and its role in slow-wave sleep. Nature 578:413-418.

Norman AB, Battaglia G, and Creese I (1985) [3H]WB4101 labels the 5-HT1A serotonin receptor subtype in rat brain. Guanine nucleotide and divalent cation sensitivity. Mol Pharmacol 28:487-494.

Nour MM, Evans L, and Carhart-Harris RL (2017) Psychedelics, personality and political perspectives. J Psychoactive Drugs 49:182-191.

Nugent AC, Ballard ED, Gould TD, Park LT, Moaddel R, Brutsche NE, and Zarate CA Jr (2019) Ketamine has distinct electrophysiological and behavioral effects in depressed and healthy subjects. Mol Psychiatry 24:1040-1052.

Nuti A, Ceravolo R, Piccinni A, Dell'Agnello G, Bellini G, Gambaccini G, Rossi C, Logi C, Dell'Osso L, and Bonuccelli U (2004) Psychiatric comorbidity in a population of Parkinson's disease patients. Eur J Neurol 11:315-320.

Nutt D and Carhart-Harris R (2020) The current status of psychedelics in psychiatry. JAMA Psychiatry DOI: 10.1001/jamapsychiatry.2020.2171 [published ahead of print]. Nuyen J, Schellevis FG, Satariano WA, Spreeuwenberg PM, Birkner MD, van den Bos GA, and Groenewegen PP (2006) Comorbidity was associated with neurologic and psychiatric diseases: a general practice-based controlled study. J Clin Epidemiol 59:1274-1284.

O'Loinsigh ED, Boland G, Kelly JP, and O'Boyle KM (2001) Behavioural, hyperthermic and neurotoxic effects of 3,4-methylenedioxymethamphetamine analogues in the Wistar rat. Prog Neuropsychopharmacol Biol Psychiatry 25: $621-638$

O’Mathúna B, Farré M, Rostami-Hodjegan A, Yang J, Cuyàs E, Torrens M, Pardo R, Abanades S, Maluf S, Tucker GT, et al. (2008) The consequences of 3,4-methylenedioxymethamphetamine induced CYP2D6 inhibition in humans. J Clin Psychopharmacol 28:523-529.

O'Neill MJ, Bleakman D, Zimmerman DM, and Nisenbaum ES (2004) AMPA receptor potentiators for the treatment of CNS disorders. Curr Drug Targets CNS Neurol Disord 3:181-194.

O'Reilly PO and Funk A (1964) Lsd in chronic alcoholism. Can Psychiatr Assoc J 9: 258-261.

Olney JW, Newcomer JW, and Farber NB (1999) NMDA receptor hypofunction model of schizophrenia. J Psychiatr Res 33:523-533.

Olson CR and Graybiel AM (1980) Sensory maps in the claustrum of the cat. Nature 288:479-481.

Ona G, Kohek M, Massaguer T, Gomariz A, Jiménez DF, Dos Santos RG, Hallak JEC, Alcázar-Córcoles MA, and Bouso JC (2019) Ayahuasca and public health: 
health status, psychosocial well-being, lifestyle, and coping strategies in a large sample of ritual ayahuasca users. J Psychoactive Drugs 51:135-145.

Ono Y, Tanaka H, Takata M, Nagahara Y, Noda Y, Tsuruma K, Shimazawa M Hozumi I, and Hara H (2014) SA4503, a sigma-1 receptor agonist, suppresses motor neuron damage in in vitro and in vivo amyotrophic lateral sclerosis models. Neurosci Lett 559:174-178.

Oquendo MA, Hastings RS, Huang YY, Simpson N, Ogden RT, Hu XZ, Goldman D, Arango V, Van Heertum RL, Mann JJ, et al. (2007) Brain serotonin transporter binding in depressed patients with bipolar disorder using positron emission tomography. Arch Gen Psychiatry 64:201-208.

Orejarena MJ, Lanfumey L, Maldonado R, and Robledo P (2011) Involvement of 5HT2A receptors in MDMA reinforcement and cue-induced reinstatement of MDMA-seeking behaviour. Int J Neuropsychopharmacol 14:927-940.

Orsolini L, Papanti GD, De Berardis D, Guirguis A, Corkery JM, and Schifano F (2017) The "Endless Trip" among the NPS Users: psychopathology and psychopharmacology in the hallucinogen-persisting perception disorder. A systematic review. Front Psychiatry 8:240.

Ortíz-Rentería M, Juárez-Contreras R, González-Ramírez R, Islas LD, Sierra-Ramírez F, Llorente I, Simon SA, Hiriart M, Rosenbaum T, and Morales-Lázaro SL (2018) TRPV1 channels and the progesterone receptor Sig-1R interact to regulate pain. Proc Natl Acad Sci USA 115:E1657-E1666.

Osmond H and Smythies J (1952) Schizophrenia: a new approach. J Ment Sci 98 309-315.

Osório FdeL, Sanches RF, Macedo LR, Santos RG, Maia-de-Oliveira JP, Wichert-Ana L, Araujo DB, Riba J, Crippa JA, and Hallak JE (2015) Antidepressant effects of a single dose of ayahuasca in patients with recurrent depression: a preliminary report. Br J Psychiatry 37:13-20.

Østby Y, Walhovd KB, Tamnes CK, Grydeland H, Westlye LT, and Fjell AM (2012) Mental time travel and default-mode network functional connectivity in the developing brain. Proc Natl Acad Sci USA 109:16800-16804.

Oye I, Paulsen O, and Maurset A (1992) Effects of ketamine on sensory perception: evidence for a role of N-methyl-D-aspartate receptors. J Pharmacol Exp Ther 260 $1209-1213$

Pachmerhiwala R, Bhide N, Straiko M, and Gudelsky GA (2010) Role of serotonin and/or norepinephrine in the MDMA-induced increase in extracellular glucose and glycogenolysis in the rat brain. Eur J Pharmacol 644:67-72.

Pacifici R, Zuccaro P, Farré M, Pichini S, Di Carlo S, Roset PN, Lopez CH, Ortuño J, Segura J, Camí J, et al. (2000) Immunomodulating activity of MDMA. Ann NY Acad Sci 914:215-224.

Pacifici R, Zuccaro P, Farré M, Pichini S, Di Carlo S, Roset PN, Ortuño J, Segura J, and de la Torre R (1999) Immunomodulating properties of MDMA alone and in combination with alcohol: a pilot study. Life Sci 65:PL309-PL316.

Pacifici R, Zuccaro P, Farré M, Poudevida S, Abanades S, Pichini S, Langohr K, Segura J, and de la Torre R (2007) Combined immunomodulating properties of 3,4 methylenedioxymethamphetamine (MDMA) and cannabis in humans. Addiction 102:931-936.

Pacifici R, Zuccaro P, Hernandez López C, Pichini S, Di Carlo S, Farré M, Roset PN, Ortuño J, Segura J, and Torre RL (2001) Acute effects of 3,4-methylenedioxymethamphetamine alone and in combination with ethanol on the immune system in humans. J Pharmacol Exp Ther 296:207-215.

Pähkla R, Kask A, and Rägo L (2000) Differential effects of beta-carbolines and antidepressants on rat exploratory activity in the elevated zero-maze. Pharmacol Biochem Behav 65:737-742.

Pahnke WN, Kurland AA, Unger S, Savage C, and Grof S (1970) The experimental use of psychedelic (LSD) psychotherapy. JAMA 212:1856-1863.

Pal A, Fontanilla D, Gopalakrishnan A, Chae YK, Markley JL, and Ruoho AE (2012) The sigma-1 receptor protects against cellular oxidative stress and activates an tioxidant response elements. Eur J Pharmacol 682:12-20

Palhano-Fontes F, Andrade KC, Tofoli LF, Santos AC, Crippa JA, Hallak JE, Ribeiro S, and de Araujo DB (2015) The psychedelic state induced by ayahuasca modulates the activity and connectivity of the default mode network. PLoS One 10:e0118143.

Palhano-Fontes F, Barreto D, Onias H, Andrade KC, Novaes MM, Pessoa JA, MotaRolim SA, Osório FL, Sanches R, Dos Santos RG, et al. (2019) Rapid antidepressant effects of the psychedelic ayahuasca in treatment-resistant depression a randomized placebo-controlled trial. Psychol Med 49:655-663.

Palmer GC and Burks TF (1971) Central and peripheral adrenergic blocking actions of LSD and BOL. Eur $J$ Pharmacol 16:113-116.

Palomino A, Vallejo-Illarramendi A, González-Pinto A, Aldama A, González-Gómez C, Mosquera F, González-García G, and Matute C (2006) Decreased levels of plasma BDNF in first-episode schizophrenia and bipolar disorder patients. Schizophr Res 86:321-322.

Pandya NJ, Klaassen RV, van der Schors RC, Slotman JA, Houtsmuller A, Smit AB, and $\mathrm{Li} \mathrm{KW} \mathrm{(2016)} \mathrm{Group} 1$ metabotropic glutamate receptors 1 and 5 form a protein complex in mouse hippocampus and cortex. Proteomics 16:2698-2705.

Pang PS, Butler J, Collins SP, Cotter G, Davison BA, Ezekowitz JA, Filippatos G Levy PD, Metra M, Ponikowski P, et al. (2017) Biased ligand of the angiotensin II type 1 receptor in patients with acute heart failure: a randomized, double-blind, placebo-controlled, phase IIB, dose ranging trial (BLAST-AHF). Eur Heart $J$ 38: 2364-2373.

Panksepp J (1992) Oxytocin effects on emotional processes: separation distress, social bonding, and relationships to psychiatric disorders. Ann N Y Acad Sci 652 243-252.

Pantoni MM and Anagnostaras SG (2019) Cognitive effects of MDMA in laboratory animals: a systematic review focusing on dose. Pharmacol Rev 71:413-449.

Pardo-Lozano R, Farré M, Yubero-Lahoz S, O'Mathúna B, Torrens M, Mustata C, Pérez-Mañá C, Langohr K, Cuyàs E, Carbó Ml, et al. (2012) Clinical pharmacology of 3,4-methylenedioxymethamphetamine (MDMA, "ecstasy"): the influence of gender and genetics (CYP2D6, COMT, 5-HTT). PLoS One 7:e47599.

Pariante CM and Lightman SL (2008) The HPA axis in major depression: classical theories and new developments. Trends Neurosci 31:464-468.
Parker KJ, Oztan O, Libove RA, Sumiyoshi RD, Jackson LP, Karhson DS, Summers JE, Hinman KE, Motonaga KS, Phillips JM, et al. (2017) Intranasal oxytocin treatment for social deficits and biomarkers of response in children with autism. Proc Natl Acad Sci USA 114:8119-8124.

Parrott AC (2001) Human psychopharmacology of Ecstasy (MDMA): a review of 15 years of empirical research. Hum Psychopharmacol 16:557-577.

Parrott AC (2002) Recreational Ecstasy/MDMA, the serotonin syndrome, and serotonergic neurotoxicity. Pharmacol Biochem Behav 71:837-844.

Parrott AC (2013) Human psychobiology of MDMA or 'Ecstasy': an overview of 25 years of empirical research. Hum Psychopharmacol 28:289-307.

Parrott AC (2014) MDMA is certainly damaging after 25 years of empirical research: a reply and refutation of Doblin et al. (2014). Hum Psychopharmacol 29:109-119.

Paruchuri P, Godkar D, Anandacoomarswamy D, Sheth K, and Niranjan S (2006) Rare case of serotonin syndrome with therapeutic doses of paroxetine. Am J Ther 13:550-552.

Pasquini L, Palhano-Fontes F, and Araujo DB (2020) Subacute effects of the psychedelic ayahuasca on the salience and default mode networks. J Psychopharmacol 34:623-635.

Passie T, Halpern JH, Stichtenoth DO, Emrich HM, and Hintzen A (2008) The pharmacology of lysergic acid diethylamide: a review. CNS Neurosci Ther 14:295-314.

Passie T, Seifert J, Schneider U, and Emrich HM (2002) The pharmacology of psilocybin. Addict Biol 7:357-364.

Passos IC, Vasconcelos-Moreno MP, Costa LG, Kunz M, Brietzke E, Quevedo J, Salum G, Magalhães PV, Kapczinski F, and Kauer-Sant'Anna M (2015) Inflammatory markers in post-traumatic stress disorder: a systematic review, metaanalysis, and meta-regression. Lancet Psychiatry 2:1002-1012.

Paterson NE, Darby WC, and Sandhu PS (2015) N,N-Dimethyltryptamine-induced psychosis. Clin Neuropharmacol 38:141-143.

Paulsen O and Moser EI (1998) A model of hippocampal memory encoding and retrieval: GABAergic control of synaptic plasticity. Trends Neurosci 21:273-278.

Peedicayil J (2019) Pharmacoepigenetics and pharmacoepigenomics: an overview. Curr Drug Discov Technol 16:392-399.

Peng W and Simantov R (2003) Altered gene expression in frontal cortex and midbrain of 3,4-methylenedioxymethamphetamine (MDMA) treated mice: differential regulation of GABA transporter subtypes. J Neurosci Res 72:250-258.

Penn E and Tracy DK (2012) The drugs don't work? antidepressants and the current and future pharmacological management of depression. Ther Adv Psychopharmacol 2:179-188.

Perez-Aguilar JM, Shan J, LeVine MV, Khelashvili G, and Weinstein H (2014) A functional selectivity mechanism at the serotonin-2A GPCR involves liganddependent conformations of intracellular loop 2. J Am Chem Soc 136:16044-16054

Perreault ML, Hasbi A, O'Dowd BF, and George SR (2014) Heteromeric dopamine receptor signaling complexes: emerging neurobiology and disease relevance. $\mathrm{Neu}$ ropsychopharmacology 39:156-168.

Peters DA (1974) Comparison of the chronic and acute effects of D-lysergic acid diethylamide (LSD) treatment on rat brain serotonin and norepinephrine. Biochem Pharmacol 23:231-237.

Peters SK, Dunlop K, and Downar J (2016) Cortico-striatal-thalamic loop circuits of the salience network: a central pathway in psychiatric disease and treatment. Front Syst Neurosci 10:104.

Petty F (1995) GABA and mood disorders: a brief review and hypothesis. $J$ Affect Disord 34:275-281.

Peyron C, Petit JM, Rampon C, Jouvet M, and Luppi PH (1998) Forebrain afferents to the rat dorsal raphe nucleus demonstrated by retrograde and anterograde tracing methods. Neuroscience 82:443-468.

Peyrovian B, McIntyre RS, Phan L, Lui LMW, Gill H, Majeed A, Chen-Li D, Nasri F, and Rosenblat JD (2020) Registered clinical trials investigating ketamine for psychiatric disorders. J Psychiatr Res 127:1-12.

Pezawas L, Meyer-Lindenberg A, Drabant EM, Verchinski BA, Munoz KE, Kolachana BS, Egan MF, Mattay VS, Hariri AR, and Weinberger DR (2005) 5-HTTLPR polymorphism impacts human cingulate-amygdala interactions: a genetic susceptibility mechanism for depression. Nat Neurosci 8:828-834.

Pham TH, Mendez-David I, Defaix C, Guiard BP, Tritschler L, David DJ, and Gardier AM (2017) Ketamine treatment involves medial prefrontal cortex serotonin to induce a rapid antidepressant-like activity in BALB/cJ mice. Neuropharmacology 112:198-209.

Phelps J (2017) Developing guidelines and competencies for the training of psychedelic therapists. J Humanist Psychol 57:450-487.

Phillips JL, Norris S, Talbot J, Birmingham M, Hatchard T, Ortiz A, Owoeye O, Batten LA, and Blier P (2019) Single, repeated, and maintenance ketamine infusions for treatment-resistant depression: a randomized controlled trial. Am J Psychiatry 176:401-409.

Phillips WJ, Ostrovsky O, Galli RL, and Dickey S (2006) Relief of acute migraine headache with intravenous oxytocin: report of two cases. $J$ Pain Palliat Care Pharmacother 20:25-28.

Pieri L, Pieri M, and Haefely W (1974) LSD as an agonist of dopamine receptors in the striatum. Nature 252:586-588.

Pires AP, De Oliveira CD, Moura S, Dörr FA, Silva WA, and Yonamine M (2009) Gas chromatographic analysis of dimethyltryptamine and beta-carboline alkaloids in ayahuasca, an Amazonian psychoactive plant beverage. Phytochem Anal 20: 149-153.

Pisano VD, Putnam NP, Kramer HM, Franciotti KJ, Halpern JH, and Holden SC (2017) The association of psychedelic use and opioid use disorders among illicit users in the United States. $J$ Psychopharmacol 31:606-613.

Pittenger C and Duman RS (2008) Stress, depression, and neuroplasticity: a convergence of mechanisms. Neuropsychopharmacology 33:88-109.

Pizzagalli DA, Holmes AJ, Dillon DG, Goetz EL, Birk JL, Bogdan R, Dougherty DD, Iosifescu DV, Rauch SL, and Fava M (2009) Reduced caudate and nucleus accumbens response to rewards in unmedicated individuals with major depressive disorder. Am J Psychiatry 166:702-710. 
Pokorny T, Duerler P, Seifritz E, Vollenweider FX, and Preller KH (2019) LSD acutely impairs working memory, executive functions, and cognitive flexibility, but not risk-based decision-making. Psychol Med DOI: 10.1017/S0033291719002393 [published ahead of print].

Polito V and Stevenson RJ (2019) A systematic study of microdosing psychedelics. PLoS One 14:e211023.

Posner J, Marsh R, Maia TV, Peterson BS, Gruber A, and Simpson HB (2014) Reduced functional connectivity within the limbic cortico-striato-thalamo-cortical loop in unmedicated adults with obsessive-compulsive disorder. Hum Brain Mapp 35:2852-2860.

Posner J, Song I, Lee S, Rodriguez CI, Moore H, Marsh R, and Blair Simpson H (2017) Increased functional connectivity between the default mode and salience networks in unmedicated adults with obsessive-compulsive disorder. Hum Brain Mapp 38:678-687.

Pottie E, Cannaert A, and Stove CP (2020) In vitro structure-activity relationship determination of 30 psychedelic new psychoactive substances by means of $\beta$-arrestin 2 recruitment to the serotonin 2A receptor. Arch Toxicol 94:3449-3460.

Preller KH (2019) The effects of low doses of lysergic acid diethylamide in healthy humans: demystifying the microdosing of psychedelics. Biol Psychiatry 86: 736-737.

Preller KH, Burt JB, Ji JL, Schleifer CH, Adkinson BD, Stämpfli P, Seifritz E, Repovs G, Krystal JH, Murray JD, et al. (2018) Changes in global and thalamic brain connectivity in LSD-induced altered states of consciousness are attributable to the 5-HT2A receptor. eLife 7:e35082.

Preller KH, Duerler P, Burt JB, Ji JL, Adkinson B, Stämpfli P, Seifritz E, Repovš G, Krystal JH, Murray JD, et al. (2020) Psilocybin induces time-dependent changes in global functional connectivity. Biol Psychiatry 88:197-207.

Preller KH, Razi A, Zeidman P, Stämpfli P, Friston KJ, and Vollenweider FX (2019) Effective connectivity changes in LSD-induced altered states of consciousness in humans. Proc Natl Acad Sci USA 116:2743-2748.

Price RB, Nock MK, Charney DS, and Mathew SJ (2009) Effects of intravenous ketamine on explicit and implicit measures of suicidality in treatment-resistant depression. Biol Psychiatry 66:522-526.

Prochazkova L, Lippelt DP, Colzato LS, Kuchar M, Sjoerds Z, and Hommel B (2018) Exploring the effect of microdosing psychedelics on creativity in an open-label natural setting. Psychopharmacology (Berl) 235:3401-3413.

Puig MV, Celada P, Díaz-Mataix L, and Artigas F (2003) In vivo modulation of the activity of pyramidal neurons in the rat medial prefrontal cortex by 5-HT2A receptors: relationship to thalamocortical afferents. Cereb Cortex 13:870-882.

Pulendran B (2004) Modulating TH1/TH2 responses with microbes, dendritic cells, and pathogen recognition receptors. Immunol Res 29:187-196.

Quednow BB, Kometer M, Geyer MA, and Vollenweider FX (2012) Psilocybin-induced deficits in automatic and controlled inhibition are attenuated by ketanserin in healthy human volunteers. Neuropsychopharmacology 37:630-640.

Quirk MC, Sosulski DL, Feierstein CE, Uchida N, and Mainen ZF (2009) A defined network of fast-spiking interneurons in orbitofrontal cortex: responses to behavioral contingencies and ketamine administration. Front Syst Neurosci 3:13.

Raison CL, Capuron L, and Miller AH (2006) Cytokines sing the blues: inflammation and the pathogenesis of depression. Trends Immunol 27:24-31.

Raison CL and Miller AH (2013) Do cytokines really sing the blues?. Cerebrum 2013 10

Raison CL, Rutherford RE, Woolwine BJ, Shuo C, Schettler P, Drake DF, Haroon E, and Miller AH (2013) A randomized controlled trial of the tumor necrosis factor antagonist infliximab for treatment-resistant depression: the role of baseline inflammatory biomarkers. JAMA Psychiatry 70:31-41.

Rakofsky JJ, Ressler KJ, and Dunlop BW (2012) BDNF function as a potential mediator of bipolar disorder and post-traumatic stress disorder comorbidity. $\mathrm{Mol}$ Psychiatry 17:22-35.

Raote I, Bhattacharyya S, and Panicker MM (2013) Functional selectivity in serotonin receptor 2A (5-HT2A) endocytosis, recycling, and phosphorylation. Mol Pharmacol 83:42-50.

Ravassard P, Hamieh AM, Malleret G, and Salin P-A (2015) Paradoxical sleep: a vigilance state to gate long-term brain plasticity?. Neurobiol Learn Mem 122 $4-10$

Ray TS (2010) Psychedelics and the human receptorome. PLoS One 5:e9019.

Redfield MM, Nicholson WJ, Edwards WD, and Tajik AJ (1992) Valve disease associated with ergot alkaloid use: echocardiographic and pathologic correlations. Ann Intern Med 117:50-52.

Reinhart V, Bove SE, Volfson D, Lewis DA, Kleiman RJ, and Lanz TA (2015) Evaluation of TrkB and BDNF transcripts in prefrontal cortex, hippocampus, and striatum from subjects with schizophrenia, bipolar disorder, and major depressive disorder. Neurobiol Dis 77:220-227.

Reissig CJ, Eckler JR, Rabin RA, and Winter JC (2005) The 5-HT1A receptor and the stimulus effects of LSD in the rat. Psychopharmacology (Berl) 182:197-204.

Reiter E, Ahn S, Shukla AK, and Lefkowitz RJ (2012) Molecular mechanism of $\beta$-arrestin-biased agonism at seven-transmembrane receptors. Annu Rev Pharmacol Toxicol 52:179-197.

Rempel NL, Callaway CW, and Geyer MA (1993) Serotonin1B receptor activation mimics behavioral effects of presynaptic serotonin release. Neuropsychopharmacology 8:201-211.

Reneman L, Booij J, Lavalaye J, de Bruin K, Reitsma JB, Gunning B, den Heeten GJ, and van Den Brink W (2002a) Use of amphetamine by recreational users of ecstasy (MDMA) is associated with reduced striatal dopamine transporter densities: a [123I]beta-CIT SPECT study--preliminary report. Psychopharmacology (Berl) 159:335-340.

Reneman L, Booij J, Majoie CB, Van Den Brink W, and Den Heeten GJ (2001a) Investigating the potential neurotoxicity of Ecstasy (MDMA): an imaging approach. Hum Psychopharmacol 16:579-588.

Reneman L, Endert E, de Bruin K, Lavalaye J, Feenstra MG, de Wolff FA, and Booij $\mathrm{J}(2002 \mathrm{~b})$ The acute and chronic effects of MDMA ("ecstasy") on cortical 5-HT2A receptors in rat and human brain. Neuropsychopharmacology 26:387-396.
Reneman L, Majoie CB, Habraken JB, and den Heeten GJ (2001b) Effects of ecstasy (MDMA) on the brain in abstinent users: initial observations with diffusion and perfusion MR imaging. Radiology 220:611-617.

Renner U, Zeug A, Woehler A, Niebert M, Dityatev A, Dityateva G, Gorinski N, Guseva D, Abdel-Galil D, Fröhlich M, et al. (2012) Heterodimerization of serotonin receptors 5-HT1A and 5-HT7 differentially regulates receptor signalling and trafficking. $J$ Cell Sci 125:2486-2499.

Riba J, Romero S, Grasa E, Mena E, Carrió I, and Barbanoj MJ (2006) Increased frontal and paralimbic activation following ayahuasca, the pan-Amazonian inebriant. Psychopharmacology (Berl) 186:93-98.

Riba J, Valle M, Urbano G, Yritia M, Morte A, and Barbanoj MJ (2003) Human pharmacology of ayahuasca: subjective and cardiovascular effects, monoamine metabolite excretion, and pharmacokinetics. J Pharmacol Exp Ther 306:73-83.

Ricaurte GA, Yuan J, Hatzidimitriou G, Cord BJ, and McCann UD (2002) Severe dopaminergic neurotoxicity in primates after a common recreational dose regimen of MDMA ("ecstasy"). Science 297:2260-2263. [Retraction published in Science (2003) 301:1479.

Richards WA (2016) Psychedelic psychotherapy: insights from 25 years of research. J Humanist Psychol 57:323-337.

Richtand NM, Welge JA, Logue AD, Keck PE Jr, Strakowski SM, and McNamara RK (2007) Dopamine and serotonin receptor binding and antipsychotic efficacy. Neuropsychopharmacology 32:1715-1726.

Rickli A, Luethi D, Reinisch J, Buchy D, Hoener MC, and Liechti ME (2015) Receptor interaction profiles of novel N-2-methoxybenzyl (NBOMe) derivatives of 2,5dimethoxy-substituted phenethylamines (2C drugs). Neuropharmacology 99 546-553.

Rickli A, Moning OD, Hoener MC, and Liechti ME (2016) Receptor interaction profiles of novel psychoactive tryptamines compared with classic hallucinogens. Eur Neuropsychopharmacol 26:1327-1337.

Riedlinger TJ and Riedlinger JE (1994) Psychedelic and entactogenic drugs in the treatment of depression. J Psychoactive Drugs 26:41-55.

Rifkin BD, Maraver MJ, and Colzato LS (2020) Microdosing psychedelics as cognitive and emotional enhancers. Psychology of Consciousness: Theory, Research, and Practice 7:316-329.

Riga MS, Lladó-Pelfort L, Artigas F, and Celada P (2018) The serotonin hallucinogen 5$\mathrm{MeO}-\mathrm{DMT}$ alters cortico-thalamic activity in freely moving mice: regionally-selective involvement of 5- $\mathrm{HT}_{1 \mathrm{~A}}$ and 5- $\mathrm{HT}_{2 \mathrm{~A}}$ receptors. Neuropharmacology 142:219-230.

Riga MS, Soria G, Tudela R, Artigas F, and Celada P (2014) The natural hallucinogen 5-MeO-DMT, component of Ayahuasca, disrupts cortical function in rats: reversal by antipsychotic drugs. Int J Neuropsychopharmacol 17:1269-1282.

Riley NM and Coon JJ (2016) Phosphoproteomics in the age of rapid and deep proteome profiling. Anal Chem 88:74-94.

Rincón-Cortés M and Grace AA (2017) Sex-dependent effects of stress on immobility behavior and vta dopamine neuron activity: modulation by ketamine. Int $J$ Neuropsychopharmacol 20:823-832.

Risbrough VB, Masten VL, Caldwell S, Paulus MP, Low MJ, and Geyer MA (2006 Differential contributions of dopamine D1, D2, and D3 receptors to MDMA-induced effects on locomotor behavior patterns in mice. Neuropsychopharmacology $\mathbf{3 1}$ 2349-2358.

Rivolta D, Heidegger T, Scheller B, Sauer A, Schaum M, Birkner K, Singer W, Wibral M, and Uhlhaas PJ (2015) Ketamine dysregulates the amplitude and connectivity of high-frequency oscillations in cortical-subcortical networks in humans: evidence from resting-state magnetoencephalography-recordings. Schizophr Bull $\mathbf{4 1}$ 1105-1114.

Robson MJ, Elliott M, Seminerio MJ, and Matsumoto RR (2012) Evaluation of sigma $(\sigma)$ receptors in the antidepressant-like effects of ketamine in vitro and in vivo. Eur Neuropsychopharmacol 22:308-317.

Rodríguez JJ, Noristani HN, Hoover WB, Linley SB, and Vertes RP (2011) Serotonergic projections and serotonin receptor expression in the reticular nucleus of the thalamus in the rat. Synapse 65:919-928.

Rogawski MA and Aghajanian GK (1979) Response of central monoaminergic neurons to lisuride: comparison with LSD. Life Sci 24:1289-1297.

Rogers GB, Keating DJ, Young RL, Wong ML, Licinio J, and Wesselingh S (2016) From gut dysbiosis to altered brain function and mental illness: mechanisms and pathways. Mol Psychiatry 21:738-748.

Rohleder N, Joksimovic L, Wolf JM, and Kirschbaum C (2004) Hypocortisolism and increased glucocorticoid sensitivity of pro-Inflammatory cytokine production in Bosnian war refugees with posttraumatic stress disorder. Biol Psychiatry 55 745-751.

Rollema H, Lu Y, Schmidt AW, and Zorn SH (1997) Clozapine increases dopamine release in prefrontal cortex by 5 -HT1A receptor activation. Eur J Pharmacol 338 R3-R5.

Romagnani S (1997) The Th1/Th2 paradigm. Immunol Today 18:263-266.

Rosa PB, Neis VB, Ribeiro CM, Moretti M, and Rodrigues AL (2016) Antidepressantlike effects of ascorbic acid and ketamine involve modulation of GABAA and GABAB receptors. Pharmacol Rep 68:996-1001.

Roseman L, Demetriou L, Wall MB, Nutt DJ, and Carhart-Harris RL (2018) Increased amygdala responses to emotional faces after psilocybin for treatmentresistant depression. Neuropharmacology 142:263-269.

Roseman L, Haijen E, Idialu-Ikato K, Kaelen M, Watts R, and Carhart-Harris R (2019) Emotional breakthrough and psychedelics: validation of the emotional breakthrough inventory. J Psychopharmacol 33:1076-1087.

Roseman L, Leech R, Feilding A, Nutt DJ, and Carhart-Harris RL (2014) The effects of psilocybin and MDMA on between-network resting state functional connectivity in healthy volunteers. Front Hum Neurosci 8:204

Rosenbaum D, Weissman C, Anderson T, Petranker R, Dinh-Williams LA, Hui K and Hapke E (2020) Microdosing psychedelics: demographics, practices, and psychiatric comorbidities. J Psychopharmacol 34:612-622.

Rosenblat JD and McIntyre RS (2018) Efficacy and tolerability of minocycline for depression: a systematic review and meta-analysis of clinical trials. J Affect Disord 227:219-225. 
Ross S, Bossis A, Guss J, Agin-Liebes G, Malone T, Cohen B, Mennenga SE, Belser A, Kalliontzi K, Babb J, et al. (2016) Rapid and sustained symptom reduction following psilocybin treatment for anxiety and depression in patients with lifethreatening cancer: a randomized controlled trial. J Psychopharmacol 30: $1165-1180$

Roth TL, Zoladz PR, Sweatt JD, and Diamond DM (2011) Epigenetic modification of hippocampal Bdnf DNA in adult rats in an animal model of post-traumatic stress disorder. J Psychiatr Res 45:919-926.

Rothlin E (1957) Pharmacology of lysergic acid diethylamide and some of its related compounds. J Pharm Pharmacol 9:569-587.

Rothman RB, Baumann MH, Dersch CM, Romero DV, Rice KC, Carroll FI, and Partilla JS (2001) Amphetamine-type central nervous system stimulants release norepinephrine more potently than they release dopamine and serotonin. Synapse 39:32-41.

Rowe DC, Stever C, Giedinghagen LN, Gard JM, Cleveland HH, Terris ST, Mohr JH, Sherman S, Abramowitz A, and Waldman ID (1998) Dopamine DRD4 receptor polymorphism and attention deficit hyperactivity disorder. Mol Psychiatry 3: 419-426.

Rubin-Kahana RS, Hassan AN, and Le Foll B (2020) Posttraumatic stress disorder after a psychedelic experience, a case report. J Addiction Medicine, doi: 10.1097/ ADM.0000000000000734

Rucker JJH, Iliff J, and Nutt DJ (2018) Psychiatry \& the psychedelic drugs. Past, present \& future. Neuropharmacology 142:200-218.

Ryskamp DA, Zhemkov V, and Bezprozvanny I (2019) Mutational analysis of sigma-1 receptor's role in synaptic stability. Front Neurosci 13:1012.

Sakloth F, Leggett E, Moerke MJ, Townsend EA, Banks ML, and Negus SS (2019) Effects of acute and repeated treatment with serotonin 5-HT2A receptor agonist hallucinogens on intracranial self-stimulation in rats. Exp Clin Psychopharmacol 27:215-226.

Salt PJ, Barnes PK, and Beswick FJ (1979) Inhibition of neuronal and extraneuronal uptake of noradrenaline by ketamine in the isolated perfused rat heart. $\mathrm{Br}$ $J$ Anaesth 51:835-838.

Sambo DO, Lebowitz JJ, and Khoshbouei H (2018) The sigma-1 receptor as a regulator of dopamine neurotransmission: a potential therapeutic target for methamphetamine addiction. Pharmacol Ther 186:152-167.

Sambo DO, Lin M, Owens A, Lebowitz JJ, Richardson B, Jagnarine DA, Shetty M, Rodriquez M, Alonge T, Ali M, et al. (2017) The sigma-1 receptor modulates methamphetamine dysregulation of dopamine neurotransmission. Nat Commun $\mathbf{8}$ : 2228.

Sanacora G, Mason GF, Rothman DL, and Krystal JH (2002) Increased occipital cortex GABA concentrations in depressed patients after therapy with selective serotonin reuptake inhibitors. Am J Psychiatry 159:663-665.

Sanacora G, Zarate CA, Krystal JH, and Manji HK (2008) Targeting the glutamatergic system to develop novel, improved therapeutics for mood disorders. Nat Rev Drug Discov 7:426-437.

Sanches RF, de Lima Osório F, Dos Santos RG, Macedo LR, Maia-de-Oliveira JP, Wichert-Ana L, de Araujo DB, Riba J, Crippa JA, and Hallak JE (2016) Antidepressant effects of a single dose of ayahuasca in patients with recurrent depression: a SPECT Study. J Clin Psychopharmacol 36:77-81.

Sara SJ (2009) The locus coeruleus and noradrenergic modulation of cognition. Nat Rev Neurosci 10:211-223.

Sarkar A and Kabbaj M (2016) Sex differences in effects of ketamine on behavior, spine density, and synaptic proteins in socially isolated rats. Biol Psychiatry 80: $448-456$.

Savić Vujović K, Vučković S, Vasović D, Medić B, Stojanović R, Divac N, Srebro D, and Prostran M (2019) Involvement of serotonergic and opioidergic systems in the antinociceptive effect of ketamine-magnesium sulphate combination in formalin test in rats. Pharmacol Rep 71:1014-1019.

Savitz J (2020) The kynurenine pathway: a finger in every pie. Mol Psychiatry 25: 131-147.

Savitz J, Lucki I, and Drevets WC (2009) 5-HT(1A) receptor function in major depressive disorder. Prog Neurobiol 88:17-31.

Schaefer A, Burmann I, Regenthal R, Arélin K, Barth C, Pampel A, Villringer A, Margulies DS, and Sacher J (2014) Serotonergic modulation of intrinsic functiona connectivity. Curr Biol 24:2314-2318.

Schindler EA, Dave KD, Smolock EM, Aloyo VJ, and Harvey JA (2012) Serotonergic and dopaminergic distinctions in the behavioral pharmacology of ( \pm )-1-(2,5-dimethoxy-4-iodophenyl)-2-aminopropane (DOI) and lysergic acid diethylamide (LSD). Pharmacol Biochem Behav 101:69-76.

Schindler EAD, Wallace RM, Sloshower JA, and D'Souza DC (2018) Neuroendocrine associations underlying the persistent therapeutic effects of classic serotonergic psychedelics. Front Pharmacol 9:177.

Schmaal L, Hibar DP, Sämann PG, Hall GB, Baune BT, Jahanshad N, Cheung JW, van Erp TGM, Bos D, Ikram MA, et al. (2017) Cortical abnormalities in adults and adolescents with major depression based on brain scans from 20 cohorts worldwide in the ENIGMA Major Depressive Disorder Working Group. Mol Psychiatry 22:900-909.

Schmid CL, Raehal KM, and Bohn LM (2008) Agonist-directed signaling of the serotonin 2A receptor depends on beta-arrestin-2 interactions in vivo. Proc Natl Acad Sci USA 105:1079-1084.

Schmid Y, Enzler F, Gasser P, Grouzmann E, Preller KH, Vollenweider FX, Brenneisen R, Müller F, Borgwardt S, and Liechti ME (2015a) Acute effects of lysergic acid diethylamide in healthy subjects. Biol Psychiatry 78:544-553.

Schmid Y, Gasser P, Oehen P, and Liechti ME (2020) Acute subjective effects in LSDand MDMA-assisted psychotherapy. J Psychopharmacol, doi: doi.org/10.1177/ 0269881120959604.

Schmid Y and Liechti ME (2018) Long-lasting subjective effects of LSD in normal subjects. Psychopharmacology (Berl) 235:535-545.

Schmid Y, Rickli A, Schaffner A, Duthaler U, Grouzmann E, Hysek CM, and Liechti ME (2015b) Interactions between bupropion and 3,4-methylenedioxymethamphetamine in healthy subjects. J Pharmacol Exp Ther 353:102-111.
Schmidt CJ (1987) Neurotoxicity of the psychedelic amphetamine, methylenedioxymethamphetamine. $J$ Pharmacol Exp Ther 240:1-7.

Schmidt CJ, Levin JA, and Lovenberg W (1987) In vitro and in vivo neurochemical effects of methylenedioxymethamphetamine on striatal monoaminergic systems in the rat brain. Biochem Pharmacol 36:747-755.

Schmidt CJ, Sorensen SM, Kehne JH, Carr AA, and Palfreyman MG (1995) The role of 5-HT2A receptors in antipsychotic activity. Life Sci 56:2209-2222.

Schmidt CJ and Taylor VL (1987) Depression of rat brain tryptophan hydroxylase activity following the acute administration of methylenedioxymethamphetamine. Biochem Pharmacol 36:4095-4102.

Schmidt HR, Zheng S, Gurpinar E, Koehl A, Manglik A, and Kruse AC (2016) Crystal structure of the human $\sigma 1$ receptor. Nature 532:527-530.

Schnoebel R, Wolff M, Peters SC, Bräu ME, Scholz A, Hempelmann G, Olschewski H, and Olschewski A (2005) Ketamine impairs excitability in superficial dorsal horn neurones by blocking sodium and voltage-gated potassium currents. $\mathrm{Br}$ $J$ Pharmacol 146:826-833.

Schwienteck KL, Li G, Poe MM, Cook JM, Banks ML, and Stevens Negus S (2017) Abuse-related effects of subtype-selective $\mathrm{GABA}_{\mathrm{A}}$ receptor positive allosteric modulators in an assay of intracranial self-stimulation in rats. Psychopharmacology (Berl) 234:2091-2101.

Scotton WJ, Hill LJ, Williams AC, and Barnes NM (2019) Serotonin syndrome: pathophysiology, clinical features, management, and potential future directions. Int J Tryptophan Res 12:1178646919873925.

Scruggs JL, Patel S, Bubser M, and Deutch AY (2000) DOI-Induced activation of the cortex: dependence on 5-HT2A heteroceptors on thalamocortical glutamatergic neurons. $J$ Neurosci 20:8846-8852.

Scruggs JL, Schmidt D, and Deutch AY (2003) The hallucinogen 1-[2,5-dimethoxy-4iodophenyl]-2-aminopropane (DOI) increases cortical extracellular glutamate levels in rats. Neurosci Lett 346:137-140.

Seeman P and Kapur S (2003) Anesthetics inhibit high-affinity states of dopamine D2 and other G-linked receptors. Synapse 50:35-40.

Seeman P, Ko F, and Tallerico T (2005) Dopamine receptor contribution to the action of PCP, LSD and ketamine psychotomimetics. Mol Psychiatry 10:877-883.

Seo MK, Hien LT, Park MK, Choi AJ, Seog D-H, Kim S-H, Park SW, and Lee JG (2020) AMPA receptor-mTORC1 signaling activation is required for neuroplastic effects of LY341495 in rat hippocampal neurons. Sci Rep 10:993.

Sessa B, Higbed L, and Nutt D (2019) A review of 3,4-methylenedioxymethamphetamine (MDMA)-assisted psychotherapy. Front Psychiatry 10:138.

Setiawan E, Wilson AA, Mizrahi R, Rusjan PM, Miler L, Rajkowska G, Suridjan I, Kennedy JL, Rekkas PV, Houle S, et al. (2015) Role of translocator protein density, a marker of neuroinflammation, in the brain during major depressive episodes. JAMA Psychiatry 72:268-275.

Shah UH, Gaitonde SA, Moreno JL, Glennon RA, Dukat M, and González-Maeso J (2019) Revised pharmacophore model for $5-\mathrm{HT}_{2 \mathrm{~A}}$ receptor antagonists derived from the atypical antipsychotic agent risperidone. ACS Chem Neurosci 10: 2318-2331.

Shajib MS and Khan WI (2015) The role of serotonin and its receptors in activation of immune responses and inflammation. Acta Physiol (Oxf) 213:561-574.

Sheline YI, Barch DM, Price JL, Rundle MM, Vaishnavi SN, Snyder AZ, Mintun MA, Wang S, Coalson RS, and Raichle ME (2009) The default mode network and selfreferential processes in depression. Proc Natl Acad Sci USA 106:1942-1947.

Shelton RC, Sanders-Bush E, Manier DH, and Lewis DA (2009) Elevated 5-HT 2A receptors in postmortem prefrontal cortex in major depression is associated with reduced activity of protein kinase A. Neuroscience 158:1406-1415.

Shen HW, Jiang XL, Winter JC, and Yu AM (2010) Psychedelic 5-methoxy-N,Ndimethyltryptamine: metabolism, pharmacokinetics, drug interactions, and pharmacological actions. Curr Drug Metab 11:659-666.

Shimizu E, Hashimoto K, Okamura N, Koike K, Komatsu N, Kumakiri C, Nakazato M, Watanabe H, Shinoda N, Okada S, et al. (2003a) Alterations of serum levels of brain-derived neurotrophic factor (BDNF) in depressed patients with or without antidepressants. Biol Psychiatry 54:70-75.

Shimizu E, Hashimoto K, Watanabe H, Komatsu N, Okamura N, Koike K, Shinoda N, Nakazato M, Kumakiri C, Okada S, et al. (2003b) Serum brain-derived neurotrophic factor (BDNF) levels in schizophrenia are indistinguishable from controls. Neurosci Lett 351:111-114.

Shokry IM, Shields CJ, Callanan JJ, Ma Z, and Tao R (2019) Differential role of dose and environment in initiating and intensifying neurotoxicity caused by MDMA in rats. BMC Pharmacol Toxicol 20:47.

Shuto T, Kuroiwa M, Sotogaku N, Kawahara Y, Oh S, Jang JH, Shin CH, Ohnishi Y, Hanada Y, Miyakawa T, et al. (2020) Obligatory roles of dopamine D1 receptors in the dentate gyrus in antidepressant actions of a selective serotonin reuptake inhibitor, fluoxetine. Molecular Psychiatry 29:1229-1244.

Siegel RK (1984) LSD-induced effects in elephants: comparisons with musth behavior. Bull Psychon Soc 22:53-56.

Silbergeld EK and Hruska RE (1979) Lisuride and LSD: dopaminergic and serotonergic interactions in the "serotonin syndrome". Psychopharmacology (Berl) 65: 233-237.

Silberstein SD (2001) Shared mechanisms and comorbidities in neurologic and psychiatric disorders. Headache 41 (Suppl 1):S11-S17.

Singh A, Puskoor S, and Saunders R (2019) Prolonged lysergic acid diethylmide induced serotonin syndrome (Abstract). Chest 156(4 Suppl):A2132.

Simantov R and Peng W (2004) MDMA (Ecstasy) controls in concert a group of genes involved in GABA neurotransmission. FEBS Lett 563:3-6.

Simmler LD, Hysek CM, and Liechti ME (2011) Sex differences in the effects of MDMA (ecstasy) on plasma copeptin in healthy subjects. J Clin Endocrinol Metab 96:2844-2850.

Sinyor M, Williams M, Belo S, Orser B, Vincent M, Mah L, Zarate C Jr, Castel S, Levitt AJ, and Schaffer A (2018) Ketamine augmentation for major depressive disorder and suicidal ideation: preliminary experience in an inpatient psychiatry setting. J Affect Disord 241:103-109. 
Skryabin VY, Vinnikova M, Nenastieva A, and Alekseyuk V (2018) Hallucinogen persisting perception disorder: a literature review and three case reports. $J$ Addict Dis 37:268-278.

Sloshower J (2018) Integrating psychedelic medicines and psychiatry: theory and methods of a model clinic, in Plant Medicines, Healing and Psychedelic Science: Cultural Perspectives (Labate BC and Cavnar C eds) pp 113-132, Springer International Publishing, Cham, Switzerland.

Smart RG and Storm T (1964) The efficacy of Lsd in the treatment of alcoholism. $Q$ J Stud Alcohol 25:333-338.

Smigielski L, Scheidegger M, Kometer M, and Vollenweider FX (2019) Psilocybinassisted mindfulness training modulates self-consciousness and brain default mode network connectivity with lasting effects. Neuroimage 196:207-215.

Smith RL, Canton H, Barrett RJ, and Sanders-Bush E (1998) Agonist properties of N,N-dimethyltryptamine at serotonin 5-HT2A and 5-HT2C receptors. Pharmacol Biochem Behav 61:323-330.

Smith TL (1977) Increased synthesis of striatal dopamine by N,N-dimethyltryptamine. Life Sci 21:1597-1601.

Smoller JW (2016) The genetics of stress-related disorders: PTSD, depression, and anxiety disorders. Neuropsychopharmacology 41:297-319.

Smythies J, Edelstein L, and Ramachandran V (2012) Hypotheses relating to the function of the claustrum. Front Integr Nuerosci 6:53.

Sobota R, Mihara T, Forrest A, Featherstone RE, and Siegel SJ (2015) Oxytocin reduces amygdala activity, increases social interactions, and reduces anxiety-like behavior irrespective of NMDAR antagonism. Behav Neurosci 129:389-398.

Soler J, Elices M, Dominguez-Clavé E, Pascual JC, Feilding A, Navarro-Gil M, García-Campayo J, and Riba J (2018) Four weekly ayahuasca sessions lead to increases in "acceptance" capacities: a comparison study with a standard 8-week mindfulness training program. Front Pharmacol 9:224.

Southwick SM, Bremner JD, Rasmusson A, Morgan CA III, Arnsten A, and Charney DS (1999) Role of norepinephrine in the pathophysiology and treatment of posttraumatic stress disorder. Biol Psychiatry 46:1192-1204.

Spencer AM (1963) Permissive group therapy with lysergic acid diethylamide. $\mathrm{Br}$ J Psychiatry 109:37-45.

Sporinova B, Manns B, Tonelli M, Hemmelgarn B, MacMaster F, Mitchell N, Au F, Ma Z, Weaver R, and Quinn A (2019) Association of mental health disorders with health care utilization and costs among adults with chronic disease. JAMA Netw Open 2:e199910.

Sprague JE, Brutcher RE, Mills EM, Caden D, and Rusyniak DE (2004) Attenuation of 3,4-methylenedioxymethamphetamine (MDMA, Ecstasy)-induced rhabdomyolysis with alpha1- plus beta3-adrenoreceptor antagonists. $\mathrm{Br}$ J Pharmacol 142:667-670.

Stanton MD and Bardoni A (1972) Drug flashbacks: reported frequency in a military population. Am J Psychiatry 129:751-755.

Staudigl T, Zaehle T, Voges J, Hanslmayr S, Esslinger C, Hinrichs H, Schmitt FC, Heinze HJ, and Richardson-Klavehn A (2012) Memory signals from the thalamus: early thalamocortical phase synchronization entrains gamma oscillations during long-term memory retrieval. Neuropsychologia 50:3519-3527.

Steiner U and Leistner E (2018) Ergot alkaloids and their hallucinogenic potential in morning glories. Planta Med 84:751-758.

Steriade M (2003) The corticothalamic system in sleep. Front Biosci 8:d878-d899.

Stern WC, Morgane PJ, and Bronzine JD (1972) LSD: effects on sleep patterns and spiking activity in the lateral geniculate nucleus. Brain Res 41:199-204.

Steuer AE, Schmidhauser C, Schmid Y, Rickli A, Liechti ME, and Kraemer T (2015) Chiral plasma pharmacokinetics of 3,4-methylenedioxymethamphetamine and its phase I and II metabolites following controlled administration to humans. Drug Metab Dispos 43:1864-1871.

Steullet P, Cabungcal JH, Bukhari SA, Ardelt MI, Pantazopoulos H, Hamati F, Salt TE, Cuenod M, Do KQ, and Berretta S (2018) The thalamic reticular nucleus in schizophrenia and bipolar disorder: role of parvalbumin-expressing neuron networks and oxidative stress. Mol Psychiatry 23:2057-2065.

Stoker AK, Olivier B, and Markou A (2012) Involvement of metabotropic glutamate receptor 5 in brain reward deficits associated with cocaine and nicotine withdrawal and somatic signs of nicotine withdrawal. Psychopharmacology (Berl) 221:317-327.

Stolk JM, Barchas JD, Goldstein M, Boggan W, and Freedman DX (1974) A comparison of psychotomimetic drug effects on rat brain norepinephrine metabolism. $J$ Pharmacol Exp Ther 189:42-50.

Stone JM, Dietrich C, Edden R, Mehta MA, De Simoni S, Reed LJ, Krystal JH, Nutt D, and Barker GJ (2012) Ketamine effects on brain GABA and glutamate levels with 1H-MRS: relationship to ketamine-induced psychopathology. Mol Psychiatry 17:664-665.

Strajhar P, Schmid Y, Liakoni E, Dolder PC, Rentsch KM, Kratschmar DV, Odermatt A, and Liechti ME (2016) Acute effects of lysergic acid diethylamide on circulating steroid levels in healthy subjects. J Neuroendocrinol 28:12374.

Strassman RJ (1984) Adverse reactions to psychedelic drugs. A review of the literature. J Nerv Ment Dis 172:577-595.

Strassman RJ (1996) Human psychopharmacology of N,N-dimethyltryptamine. Behav Brain Res 73:121-124

Strassman RJ and Qualls CR (1994) Dose-response study of N,N-dimethyltryptamine in humans. I. Neuroendocrine, autonomic, and cardiovascular effects. Arch Gen Psychiatry 51:85-97.

Strawbridge R, Arnone D, Danese A, Papadopoulos A, Herane Vives A, and Cleare AJ (2015) Inflammation and clinical response to treatment in depression: a metaanalysis. Eur Neuropsychopharmacol 25:1532-1543.

Strosberg AD (1993) Structure, function, and regulation of adrenergic receptors. Protein Sci 2:1198-1209.

Strosberg AD (1995) Structure, function, and regulation of the three beta-adrenergic receptors. Obes Res 3 (Suppl 4):501S-505S.

Studerus E, Gamma A, Kometer M, and Vollenweider FX (2012) Prediction of psilocybin response in healthy volunteers. PLoS One 7:e30800.

Studerus E, Kometer M, Hasler F, and Vollenweider FX (2011) Acute, subacute and long-term subjective effects of psilocybin in healthy humans: a pooled analysis of experimental studies. J Psychopharmacol 25:1434-1452.
Su T-P, Su T-C, Nakamura Y, and Tsai S-Y (2016) The sigma-1 receptor as a pluripotent modulator in living systems. Trends Pharmacol Sci 37:262-278.

Sugihara S and Burnett JC Jr (2017) BLAST-AHF: insights into biased AT1 ligands and heart failure. Beginning of the end or end of the beginning?. Eur Heart $J$ 38: 2374-2376.

Surget A, Wang Y, Leman S, Ibarguen-Vargas Y, Edgar N, Griebel G, Belzung C, and Sibille E (2009) Corticolimbic transcriptome changes are state-dependent and region-specific in a rodent model of depression and of antidepressant reversal. Neuropsychopharmacology 34:1363-1380.

Svensson TH, Bunney BS, and Aghajanian GK (1975) Inhibition of both noradrenergic and serotonergic neurons in brain by the $\alpha$-adrenergic agonist clonidine. Brain Res 92:291-306.

Sweat NW, Bates LW, and Hendricks PS (2016) The associations of naturalistic classic psychedelic use, mystical experience, and creative problem solving. $J$ Psychoactive Drugs 48:344-350.

Sweeney-Reed CM, Zaehle T, Voges J, Schmitt FC, Buentjen L, Kopitzki K, Esslinger C, Hinrichs H, Heinze HJ, Knight RT, et al. (2014) Corticothalamic phase synchrony and cross-frequency coupling predict human memory formation. eLife $\mathbf{3}$ : e05352.

Swerdlow NR and Koob GF (1987) Dopamine, schizophrenia, mania, and depression: toward a unified hypothesis of cortico-striatopallido-thalamic function. Behav Brain Sci 10:197-208.

Szabo A and Frecska E (2016) Dimethyltryptamine (DMT): a biochemical Swiss Army knife in neuroinflammation and neuroprotection?. Neural Regen Res 11: 396-397.

Szabo A, Kovacs A, Frecska E, and Rajnavolgyi E (2014) Psychedelic N,N-dimethyltryptamine and 5-methoxy-N,N-dimethyltryptamine modulate innate and adaptive inflammatory responses through the sigma-1 receptor of human monocyte-derived dendritic cells. PLoS One 9:e106533.

Szabo A, Kovacs A, Riba J, Djurovic S, Rajnavolgyi E, and Frecska E (2016) The endogenous hallucinogen and trace amine N,N-dimethyltryptamine (DMT) displays potent protective effects against hypoxia via sigma-1 receptor activation in human primary iPSC-derived cortical neurons and microglia-like immune cells. Front Neurosci 10:423.

Szmulewicz AG, Valerio MP, and Smith JM (2015) Switch to mania after ayahuasca consumption in a man with bipolar disorder: a case report. Int J Bipolar Disord 3:4. Tagliazucchi E, Roseman L, Kaelen M, Orban C, Muthukumaraswamy SD, Murphy K, Laufs H, Leech R, McGonigle J, Crossley N, et al. (2016) Increased global functional connectivity correlates with LSD-induced ego dissolution. Curr Biol 26: 1043-1050.

Takahashi T, Kinoshita M, Shono S, Habu Y, Ogura T, Seki S, and Kazama T (2010) The effect of ketamine anesthesia on the immune function of mice with postoperative septicemia. Anesth Analg 111:1051-1058.

Tanaka M and Nishikawa T (1994) Oral clonidine premedication attenuates the hypertensive response to ketamine. Br J Anaesth 73:758-762.

Taneja M, Salim S, Saha K, Happe HK, Qutna N, Petty F, Bylund DB, and Eikenburg DC (2011) Differential effects of inescapable stress on locus coeruleus GRK3, alpha2-adrenoceptor and CRF1 receptor levels in learned helpless and nonhelpless rats: a potential link to stress resilience. Behav Brain Res 221:25-33.

Tang SW and Seeman P (1980) Effect of antidepressant drugs on serotonergic and adrenergic receptors. Naunyn Schmiedebergs Arch Pharmacol 311:255-261.

Tao R, Shokry IM, and Callanan JJ (2017) Environment influencing serotonin syndrome induced by ecstasy abuse. Ann Forensic Res Anal 4:1039.

Taube HD, Montel H, Hau G, and Starke K (1975) Phencyclidine and ketamine: comparison with the effect of cocaine on the noradrenergic neurones of the rat brain cortex. Naunyn Schmiedebergs Arch Pharmacol 291:47-54.

Teitler M, Leonhardt S, Appel NM, De Souza EB, and Glennon RA (1990) Receptor pharmacology of MDMA and related hallucinogens. Ann NY Acad Sci 600: 626-638, discussion 638-639.

Tewari T and Mukherjee S (2010) Microdosing: concept, application and relevance. Perspect Clin Res 1:61-63.

Thal SB, Daniels JK, and Jungaberle H (2019) The link between childhood trauma and dissociation in frequent users of classic psychedelics and dissociatives. $J$ Subst Use 24:524-531.

Thomas G, Lucas P, Capler NR, Tupper KW, and Martin G (2013) Ayahuascaassisted therapy for addiction: results from a preliminary observational study in Canada. Curr Drug Abuse Rev 6:30-42.

Thomasius R, Petersen KU, Zapletalova P, Wartberg L, Zeichner D, and Schmoldt A (2005) Mental disorders in current and former heavy ecstasy (MDMA) users. Addiction 100:1310-1319.

Thompson C and Szabo A (2020) Psychedelics as a novel approach to treating autoimmune conditions. Immunology Letters 228:45-54.

Thompson MR, Callaghan PD, Hunt GE, Cornish JL, and McGregor IS (2007) A role for oxytocin and 5-HT(1A) receptors in the prosocial effects of 3,4 methylenedioxymethamphetamine ("ecstasy"). Neuroscience 146:509-514.

Timmermann C, Spriggs MJ, Kaelen M, Leech R, Nutt DJ, Moran RJ, CarhartHarris RL, and Muthukumaraswamy SD (2018) LSD modulates effective connectivity and neural adaptation mechanisms in an auditory oddball paradigm. Neuropharmacology 142:251-262.

Tisoncik JR, Korth MJ, Simmons CP, Farrar J, Martin TR, and Katze MG (2012) Into the eye of the cytokine storm. Microbiol Mol Biol Rev 76:16-32.

Titeler M, Lyon RA, and Glennon RA (1988) Radioligand binding evidence implicates the brain 5-HT2 receptor as a site of action for LSD and phenylisopropylamine hallucinogens. Psychopharmacology (Berl) 94:213-216.

Tizabi Y, Bhatti BH, Manaye KF, Das JR, and Akinfiresoye L (2012) Antidepressantlike effects of low ketamine dose is associated with increased hippocampal AMPA NMDA receptor density ratio in female Wistar-Kyoto rats. Neuroscience 213:72-80.

Torda C (1968) Contribution to serotonin theory of dreaming (LSD infusion). NY State J Med 68:1135-1138.

Torgerson CM, Irimia A, Goh SY, and Van Horn JD (2015) The DTI connectivity of the human claustrum. Hum Brain Mapp 36:827-838. 
Touriño C, Zimmer A, and Valverde O (2010) THC prevents MDMA neurotoxicity in mice. PLoS One 5:e9143.

Tourino MC, de Oliveira EM, Bellé LP, Knebel FH, Albuquerque RC, Dörr FA, Okada SS, Migliorini S, Soares IS, and Campa A (2013) Tryptamine and dimethyltryptamine inhibit indoleamine 2,3 dioxygenase and increase the tumor-reactive effect of peripheral blood mononuclear cells. Cell Biochem Funct 31:361-364.

Traber DL, Wilson RD, and Priano LL (1970) The effect of bea-adrenergic blockade on the cardiopulmonary response to ketamine. Anesth Analg 49:604-613.

Traber DL, Wilson RD, and Priano LL (1971) The effect of alpha-adrenergic blockade on the cardiopulmonary response to ketamine. Anesth Analg 50:737-742.

Triana-Del Río R, van den Burg E, Stoop R, and Hegoburu C (2019) Acute and longlasting effects of oxytocin in cortico-limbic circuits: consequences for fear recall and extinction. Psychopharmacology (Berl) 236:339-354

Tronson NC and Taylor JR (2007) Molecular mechanisms of memory reconsolidation. Nat Rev Neurosci 8:262-275.

Troyano-Rodriguez E, Lladó-Pelfort L, Santana N, Teruel-Martí V, Celada P, and Artigas F (2014) Phencyclidine inhibits the activity of thalamic reticular gamma-aminobutyric acidergic neurons in rat brain. Biol Psychiatry 76:937-945.

Trullas R and Skolnick P (1990) Functional antagonists at the NMDA receptor complex exhibit antidepressant actions. Eur J Pharmacol 185:1-10.

Tsai HF, Wang H, Gebler JC, Poulter CD, and Schardl CL (1995) The Claviceps purpurea gene encoding dimethylallyltryptophan synthase, the committed step for ergot alkaloid biosynthesis. Biochem Biophys Res Commun 216 119-125.

Tsai SY, Chuang JY, Tsai MS, Wang XF, Xi ZX, Hung JJ, Chang WC, Bonci A, and Su TP (2015) Sigma-1 receptor mediates cocaine-induced transcriptional regulation by recruiting chromatin-remodeling factors at the nuclear envelope. Proc Natl Acad Sci USA 112:E6562-E6570.

Tsai SY, Hayashi T, Mori T, and Su TP (2009) Sigma-1 receptor chaperones and diseases. Cent Nerv Syst Agents Med Chem 9:184-189.

Tsankova N, Renthal W, Kumar A, and Nestler EJ (2007) Epigenetic regulation in psychiatric disorders. Nat Rev Neurosci 8:355-367.

Tso MM, Blatchford KL, Callado LF, McLaughlin DP, and Stamford JA (2004) Stereoselective effects of ketamine on dopamine, serotonin and noradrenaline release and uptake in rat brain slices. Neurochem Int 44:1-7.

Tsukada H, Nishiyama S, Kakiuchi T, Ohba H, Sato K, and Harada N (2001) Ketamine alters the availability of striatal dopamine transporter as measured by [(11) Clbeta-CFT and [(11)C]beta-CIT-FE in the monkey brain. Synapse 42:273-280.

Tudzynski P and Scheffer J (2004) Claviceps purpurea: molecular aspects of a unique pathogenic lifestyle. Mol Plant Pathol 5:377-388.

Turrigiano GG (2008) The self-tuning neuron: synaptic scaling of excitatory synapses. Cell 135:422-435.

Tynan RJ, Weidenhofer J, Hinwood M, Cairns MJ, Day TA, and Walker FR (2012) A comparative examination of the anti-inflammatory effects of SSRI and SNRI antidepressants on LPS stimulated microglia. Brain Behav Immun 26:469-479.

Udenfriend S, Witkop B, Redfield BG, and Weissbach H (1958) Studies with reversible inhibitors of monoamine oxidase: harmaline and related compounds. Biochem Pharmacol 1:160-165.

Umut G, Küçükparlak I, Özgen G, and Türkcan A (2011) A mood disorder episode with an onset under chronic cannabis consumption and accompanied with psychotic features immediately after N,N-dimethyltryptamine (DMT) use: a case report. Dusunen Adam Psikivatri ve Nörolojik Bilimler Dergisi 24:246-250.

Urban JD, Clarke WP, von Zastrow M, Nichols DE, Kobilka B, Weinstein H, Javitch JA, Roth BL, Christopoulos A, Sexton PM, et al. (2007) Functional selectivity and classical concepts of quantitative pharmacology. J Pharmacol Exp Ther 320:1-13.

U.S. Food and Drug Administration (2020) FDA approves new opioid for intravenous use in hospitals, other controlled clinical settings. https://www.fda.gov/.

Uthaug MV, Lancelotta R, Szabo A, Davis AK, Riba J, and Ramaekers JG (2020) Prospective examination of synthetic 5-methoxy-N,N-dimethyltryptamine in halation: effects on salivary IL-6, cortisol levels, affect, and non-judgment. Psychopharmacology (Berl) 237:773-785.

Uthaug MV, Lancelotta R, van Oorsouw K, Kuypers KPC, Mason N, Rak J, Suláková A, Jurok R, Maryška M, Kuchař M, et al. (2019) A single inhalation of vapor from dried toad secretion containing 5-methoxy-N,N-dimethyltryptamine (5-MeO-DMT) in a naturalistic setting is related to sustained enhancement of satisfaction with life, mindfulness-related capacities, and a decrement of psychopathological symptoms. Psychopharmacology (Berl) 236:2653-2666.

Uvnäs-Moberg K, Bjökstrand E, Hillegaart V, and Ahlenius S (1999) Oxytocin as a possible mediator of SSRI-induced antidepressant effects. Psychopharmacology (Berl) 142.95-101.

Uyeno ET and Benson WM (1965) Effects of lysergic acid diethylamide on attack behavior of male albino mice. Psychopharmacology (Berl) 7:20-26.

Vaidya VA, Marek GJ, Aghajanian GK, and Duman RS (1997) 5-HT2A receptormediated regulation of brain-derived neurotrophic factor mRNA in the hippocampus and the neocortex. $J$ Neurosci 17:2785-2795.

Vaiva G, Boss V, Ducrocq F, Fontaine M, Devos P, Brunet A, Laffargue P, Goudemand M, and Thomas P (2006) Relationship between posttrauma GABA plasma levels and PTSD at 1-year follow-up. Am J Psychiatry 163:1446-1448.

Valentino RJ, Foote SL, and Aston-Jones G (1983) Corticotropin-releasing factor activates noradrenergic neurons of the locus coeruleus. Brain Res 270:363-367.

Van de Kar LD, Javed A, Zhang Y, Serres F, Raap DK, and Gray TS (2001) 5-HT2A receptors stimulate ACTH, corticosterone, oxytocin, renin, and prolactin release and activate hypothalamic CRF and oxytocin-expressing cells. $J$ Neurosci 21 : $3572-3579$

Vandermaelen CP and Aghajanian GK (1983) Electrophysiological and pharmacological characterization of serotonergic dorsal raphe neurons recorded extracellularly and intracellularly in rat brain slices. Brain Res 289:109-119.

Verrico CD, Lynch L, Fahey MA, Fryer A-K, Miller GM, and Madras BK (2008) MDMA-induced impairment in primates: antagonism by a selective norepinephrine or serotonin, but not by a dopamine/norepinephrine transport inhibitor. J Psychopharmacol 22:187-202.
Verrico CD, Miller GM, and Madras BK (2007) MDMA (Ecstasy) and human dopamine, norepinephrine, and serotonin transporters: implications for MDMA-induced neurotoxicity and treatment. Psychopharmacology (Berl) 189:489-503.

Vertes RP and Hoover WB (2008) Projections of the paraventricular and paratenial nuclei of the dorsal midline thalamus in the rat. J Comp Neurol 508:212-237.

Vertes RP, Hoover WB, Do Valle AC, Sherman A, and Rodriguez JJ (2006) Efferent projections of reuniens and rhomboid nuclei of the thalamus in the rat. $J$ Comp Neurol 499:768-796.

Vidal S, Gex-Fabry M, Bancila V, Michalopoulos G, Warrot D, Jermann F, Dayer A, Sterpenich V, Schwartz S, Vutskits L, et al. (2018) Efficacy and safety of a rapid intravenous injection of ketamine $0.5 \mathrm{mg} / \mathrm{kg}$ in treatment-resistant major depression: an open 4-week longitudinal study. J Clin Psychopharmacol 38:590-597.

Vigo DV, Kestel D, Pendakur K, Thornicroft G, and Atun R (2019) Disease burden and government spending on mental, neurological, and substance use disorders, and self-harm: cross-sectional, ecological study of health system response in the Americas. Lancet Public Health 4:e89-e96.

Violin JD, Crombie AL, Soergel DG, and Lark MW (2014) Biased ligands at G-protein-coupled receptors: promise and progress. Trends Pharmacol Sci 35:308-316.

Vizeli P and Liechti ME (2018) Oxytocin receptor gene variations and socio-emotional effects of MDMA: a pooled analysis of controlled studies in healthy subjects. PLoS One 13: 0199384

Vizeli P and Liechti ME (2019) No influence of dopamine system gene variations on acute effects of MDMA. Front Psychiatry 10:755.

Vizeli P, Meyer Zu Schwabedissen HE, and Liechti ME (2018) No major role of norepinephrine transporter gene variations in the cardiostimulant effects of MDMA. Eur J Clin Pharmacol 74:275-283.

Vizeli P, Schmid Y, Prestin K, Meyer Zu Schwabedissen HE, and Liechti ME (2017) Pharmacogenetics of ecstasy: CYP1A2, CYP2C19, and CYP2B6 polymorphisms moderate pharmacokinetics of MDMA in healthy subjects. Eur Neuropsychopharmacol 27:232-238

Vlisides PE, Bel-Bahar T, Lee U, Li D, Kim H, Janke E, Tarnal V, Pichurko AB McKinney AM, Kunkler BS, et al. (2017) Neurophysiologic correlates of ketamine sedation and anesthesia: a high-density electroencephalography study in healthy volunteers. Anesthesiology 127:58-69.

Vollenweider FX (2001) Brain mechanisms of hallucinogens and entactogens. Dialogues Clin Neurosci 3:265-279.

Vollenweider FX, Csomor PA, Knappe B, Geyer MA, and Quednow BB (2007) The effects of the preferential 5-HT2A agonist psilocybin on prepulse inhibition of startle in healthy human volunteers depend on interstimulus interval. Neuropsychopharmacology 32:1876-1887.

Vollenweider FX, Gamma A, Liechti M, and Huber T (1999a) Is a single dose of MDMA harmless?. Neuropsychopharmacology 21:598-600.

Vollenweider FX and Kometer M (2010) The neurobiology of psychedelic drugs: implications for the treatment of mood disorders. Nat Rev Neurosci 11:642-651.

Vollenweider FX, Liechti ME, Gamma A, Greer G, and Geyer M (2002) Acute psychological and neurophysiological effects of MDMA in humans. J Psychoactive Drugs 34:171-184.

Vollenweider FX, Vollenweider-Scherpenhuyzen MF, Bäbler A, Vogel H, and Hell D (1998) Psilocybin induces schizophrenia-like psychosis in humans via a serotonin-2 agonist action. Neuroreport 9:3897-3902

Vollenweider FX, Vontobel P, Hell D, and Leenders KL (1999b) 5-HT modulation of dopamine release in basal ganglia in psilocybin-induced psychosis in man--a PET study with [11C]raclopride. Neuropsychopharmacology 20:424-433.

Vollenweider FX, Vontobel P, Oye I, Hell D, and Leenders KL (2000) Effects of (S)ketamine on striatal dopamine: a [11C]raclopride PET study of a model psychosis in humans. $J$ Psychiatr Res 34:35-43.

Vos T, Flaxman AD, Naghavi M, Lozano R, Michaud C, Ezzati M, Shibuya K, Salomon JA, Abdalla S, Aboyans V, et al. (2012) Years lived with disability (YLDs) for 1160 sequelae of 289 diseases and injuries 1990-2010: a systematic analysis for the Global Burden of Disease Study 2010 [published correction appears in Lancet (2013) 381:628]. Lancet 380:2163-2196.

Wacker D, Wang C, Katritch V, Han GW, Huang XP, Vardy E, McCorvy JD, Jiang Y, Chu M, Siu FY, et al. (2013) Structural features for functional selectivity at serotonin receptors. Science 340:615-619.

Wacker D, Wang S, McCorvy JD, Betz RM, Venkatakrishnan AJ, Levit A, Lansu K, Schools ZL, Che T, Nichols DE, et al. (2017) Crystal structure of an LSD-bound human serotonin receptor. Cell 168:377-389.e12.

Waldmeier PC, Delini-Stula A, and Maitre L (1976) Preferential deamination of dopamine by an A type monoamine oxidase in rat brain. Naunyn Schmiedebergs Arch Pharmacol 292:9-14.

Waldmeier PC and Maître L (1977) Neurochemical investigations of the interaction of N,N-dimethyltryptamine with dopaminergic system in rat brain. Psychopharmacology (Berl) 52:137-144.

Wan LB, Levitch CF, Perez AM, Brallier JW, Iosifescu DV, Chang LC, Foulkes A Mathew SJ, Charney DS, and Murrough JW (2015) Ketamine safety and tolerability in clinical trials for treatment-resistant depression. J Clin Psychiatry $\mathbf{7 6}$ 247-252.

Wang DS, Penna A, and Orser BA (2017a) Ketamine increases the function of $\gamma$-aminobutyric acid type A receptors in hippocampal and cortical neurons. Anesthesiology 126:666-677.

Wang Q, Ng L, Harris JA, Feng D, Li Y, Royall JJ, Oh SW, Bernard A, Sunkin SM Koch C, et al. (2017b) Organization of the connections between claustrum and cortex in the mouse. J Comp Neurol 525:1317-1346.

Wang Q, Webber RM, and Stanley GB (2010) Thalamic synchrony and the adaptive gating of information flow to cortex. Nat Neurosci 13:1534-1541.

Ward LM (2011) The thalamic dynamic core theory of conscious experience. Conscious Cogn 20:464-486.

Warren JM, Dham-Nayyar P, and Alexander J (2013) Recreational use of naturally occurring dimethyltryptamine--contributing to psychosis?. Aust N Z J Psychiatry 47:398-399. 
Watts R and Luoma JB (2020) The use of the psychological flexibility model to support psychedelic assisted therapy. J Contextual Behav Sci 15:92-102.

Watts SW, Gackenheimer SL, Gehlert DR, and Cohen ML (1994) Autoradiographic comparison of [125I]LSD-labeled 5-HT2A receptor distribution in rat and Guinea pig brain. Neurochem Int 24:565-574.

Watts VJ, Lawler CP, Fox DR, Neve KA, Nichols DE, and Mailman RB (1995) LSD and structural analogs: pharmacological evaluation at D1 dopamine receptors. Psychopharmacology (Berl) 118:401-409.

Webb M, Copes H, and Hendricks PS (2019) Narrative identity, rationality, and microdosing classic psychedelics. Int J Drug Policy 70:33-39.

Weber MD, Frank MG, Tracey KJ, Watkins LR, and Maier SF (2015) Stress induces the danger-associated molecular pattern HMGB-1 in the hippocampus of male Sprague Dawley rats: a priming stimulus of microglia and the NLRP3 inflammasome. J Neurosci 35:316-324.

Wells MF, Wimmer RD, Schmitt LI, Feng G, and Halassa MM (2016) Thalamic reticular impairment underlies attention deficit in Ptchd1(Y/-) mice. Nature 532: 58-63.

Wesnes KA, Hopkins SC, Brooker HJ, and Koblan KS (2016) Differences in memory function between 5-HT1A receptor genotypes in patients with major depressive disorder. CNS Spectr 21:379-384.

West LJ, Pierce CM, and Thomas WD (1962) Lysergic acid diethylamide: its effects on a male asiatic elephant. Science 138:1100-1103.

Whirl-Carrillo M, McDonagh EM, Hebert JM, Gong L, Sangkuhl K, Thorn CF, Altman RB, and Klein TE (2012) Pharmacogenomics knowledge for personalized medicine. Clin Pharmacol Ther 92:414-417.

White MG, Panicker M, Mu C, Carter AM, Roberts BM, Dharmasri PA, and Mathur $\mathrm{BN}$ (2018) Anterior cingulate cortex input to the claustrum is required for top-down action control. Cell Rep 22:84-95.

White PF, Schüttler J, Shafer A, Stanski DR, Horai Y, and Trevor AJ (1985) Comparative pharmacology of the ketamine isomers. Studies in volunteers. $B r$ $J$ Anaesth 57:197-203.

White SR, Duffy P, and Kalivas PW (1994) Methylenedioxymethamphetamine depresses glutamate-evoked neuronal firing and increases extracellular levels of dopamine and serotonin in the nucleus accumbens in vivo. Neuroscience 62:41-50.

White SR, Harris GC, Imel KM, and Wheaton MJ (1995) Inhibitory effects of dopamine and methylenedioxymethamphetamine (MDMA) on glutamate-evoked firing of nucleus accumbens and caudate/putamen cells are enhanced following cocaine self-administration. Brain Res 681:167-176.

Whiteford HA, Degenhardt L, Rehm J, Baxter AJ, Ferrari AJ, Erskine HE, Charlson FJ, Norman RE, Flaxman AD, Johns N, et al. (2013) Global burden of disease attributable to mental and substance use disorders: findings from the Global Burden of Disease Study 2010. Lancet 382:1575-1586.

Williams JT, Ingram SL, Henderson G, Chavkin C, von Zastrow M, Schulz S, Koch T, Evans CJ, and Christie MJ (2013) Regulation of $\mu$-opioid receptors: desensitization, phosphorylation, internalization, and tolerance. Pharmacol Rev 65: $223-254$

Williams NR, Heifets BD, Blasey C, Sudheimer K, Pannu J, Pankow H, Hawkins J, Birnbaum J, Lyons DM, Rodriguez CI, et al. (2018) Attenuation of antidepressant effects of ketamine by opioid receptor antagonism. Am J Psychiatry 175: $1205-1215$

Willins DL, Deutch AY, and Roth BL (1997) Serotonin 5-HT2A receptors are expressed on pyramidal cells and interneurons in the rat cortex. Synapse 27:79-82.

Wilson SN, van der Kolk B, Burbridge J, Fisler R, and Kradin R (1999) Phenotype of blood lymphocytes in PTSD suggests chronic immune activation. Psychosomatics 40:222-225.

Winter JC, Rice KC, Amorosi DJ, and Rabin RA (2007) Psilocybin-induced stimulus control in the rat. Pharmacol Biochem Behav 87:472-480.

Wischhof L and Koch M (2012) Pre-treatment with the mGlu2/3 receptor agonist LY379268 attenuates DOI-induced impulsive responding and regional c-Fos protein expression. Psychopharmacology (Berl) 219:387-400.

Wittchen HU, Jacobi F, Rehm J, Gustavsson A, Svensson M, Jönsson B, Olesen J, Allgulander C, Alonso J, Faravelli C, et al. (2011) The size and burden of mental disorders and other disorders of the brain in Europe 2010. Eur Neuropsychopharmacol 21:655-679.

Wolkowitz OM, Wolf J, Shelly W, Rosser R, Burke HM, Lerner GK, Reus VI, Nelson JC, Epel ES, and Mellon SH (2011) Serum BDNF levels before treatment predict SSRI response in depression. Prog Neuropsychopharmacol Biol Psychiatry 35: $1623-1630$

Wong ML, Dong C, Maestre-Mesa J, and Licinio J (2008) Polymorphisms in inflammation-related genes are associated with susceptibility to major depression and antidepressant response. Mol Psychiatry 13:800-812.

Wong ML, Inserra A, Lewis MD, Mastronardi CA, Leong L, Choo J, Kentish S, Xie P, Morrison M, Wesselingh SL, et al. (2016) Inflammasome signaling affects anxietyand depressive-like behavior and gut microbiome composition. Mol Psychiatry 21: 797-805.

Wong ML, Kling MA, Munson PJ, Listwak S, Licinio J, Prolo P, Karp B, McCutcheon IE, Geracioti TD Jr, DeBellis MD, et al. (2000) Pronounced and sustained central hypernoradrenergic function in major depression with melancholic features: relation to hypercortisolism and corticotropin-releasing hormone. Proc Natl Acad Sci USA 97:325-330.

Woodcock EA, White R, and Diwadkar VA (2015) The dorsal prefrontal and dorsal anterior cingulate cortices exert complementary network signatures during encoding and retrieval in associative memory. Behav Brain Res 290:152-160.

Wootten D, Christopoulos A, Marti-Solano M, Babu MM, and Sexton PM (2018) Mechanisms of signalling and biased agonism in G protein-coupled receptors. Nat Rev Mol Cell Biol 19:638-653.

Wray NR, Ripke S, Mattheisen M, Trzaskowski M, Byrne EM, Abdellaoui A, Adams MJ, Agerbo E, Air TM, Andlauer TMF, et al.; eQTLGen; 23andMe; Major Depressive Disorder Working Group of the Psychiatric Genomics Consortium (2018) Genome-wide association analyses identify 44 risk variants and refine the genetic architecture of major depression. Nat Genet 50:668-681.
Yagasaki Y, Numakawa T, Kumamaru E, Hayashi T, Su TP, and Kunugi H (2006) Chronic antidepressants potentiate via sigma-1 receptors the brain-derived neurotrophic factor-induced signaling for glutamate release. $J$ Biol Chem 281: 12941-12949.

Yamakura T, Chavez-Noriega LE, and Harris RA (2000) Subunit-dependent inhibition of human neuronal nicotinic acetylcholine receptors and other ligandgated ion channels by dissociative anesthetics ketamine and dizocilpine. Anesthe siology 92:1144-1153.

Yamamoto BK, Nash JF, and Gudelsky GA (1995) Modulation of methylenedioxymethamphetamine-induced striatal dopamine release by the in teraction between serotonin and gamma-aminobutyric acid in the substantia nigra $J$ Pharmacol Exp Ther 273:1063-1070.

Yamamoto BK and Spanos LJ (1988) The acute effects of methylenedioxymethamphetamine on dopamine release in the awake-behaving rat. Eur J Pharmacol 148:195-203.

Yamamoto S, Ohba H, Nishiyama S, Harada N, Kakiuchi T, Tsukada H, and Domino EF (2013) Subanesthetic doses of ketamine transiently decrease serotonin transporter activity: a PET study in conscious monkeys. Neuropsychopharmacology $\mathbf{3 8}$ : 2666-2674.

Yamanaka H, Yokoyama C, Mizuma H, Kurai S, Finnema SJ, Halldin C, Doi H, and Onoe $\mathrm{H}$ (2014) A possible mechanism of the nucleus accumbens and ventral pallidum 5-HT1B receptors underlying the antidepressant action of ketamine: a PET study with macaques. Transl Psychiatry 4:e342.

Yamanouchi Y, Iwata N, Suzuki T, Kitajima T, Ikeda M, and Ozaki N (2003) Effect of DRD2, 5-HT2A, and COMT genes on antipsychotic response to risperidone. Pharmacogenomics $J$ 3:356-361.

Yamauchi M, Miyara T, Matsushima T, and Imanishi T (2006) Desensitization of 5HT2A receptor function by chronic administration of selective serotonin reuptake inhibitors. Brain Res 1067:164-169.

Yanakieva S, Polychroni N, Family N, Williams LTJ, Luke DP, and Terhune DB (2019) The effects of microdose LSD on time perception: a randomised, doubleblind, placebo-controlled trial. Psychopharmacology (Berl) 236:1159-1170.

Yang C, Han M, Zhang JC, Ren Q, and Hashimoto K (2016) Loss of parvalbuminimmunoreactivity in mouse brain regions after repeated intermittent administration of esketamine, but not R-ketamine. Psychiatry Res 239:281-283.

Yang Y, Cui Y, Sang K, Dong Y, Ni Z, Ma S, and Hu H (2018) Ketamine blocks bursting in the lateral habenula to rapidly relieve depression. Nature 554:317-322 Yen T, Khafaja M, Lam N, Crumbacher J, Schrader R, Rask J, Billstrand M, Rothfork $\mathrm{J}$, and Abbott CC (2015) Post-electroconvulsive therapy recovery and reorientation time with methohexital and ketamine: a randomized, longitudinal, crossover design trial. J ECT 31:20-25.

Yeshurun S and Hannan AJ (2019) Transgenerational epigenetic influences of paternal environmental exposures on brain function and predisposition to psychiatric disorders. Mol Psychiatry 24:536-548.

Yeung LY, Wai MS, Fan M, Mak YT, Lam WP, Li Z, Lu G, and Yew DT (2010) Hyperphosphorylated tau in the brains of mice and monkeys with long-term administration of ketamine. Toxicol Lett 193:189-193.

Yohn CN, Gergues MM, and Samuels BA (2017) The role of 5-HT receptors in depression. Mol Brain 10:28.

Young AH (2004) Cortisol in mood disorders. Stress 7:205-208.

Young MB, Norrholm SD, Khoury LM, Jovanovic T, Rauch SAM, Reiff CM, Dunlop BW, Rothbaum BO, and Howell LL (2017) Inhibition of serotonin transporters disrupts the enhancement of fear memory extinction by 3,4-methylenedioxymethamphetamine (MDMA). Psychopharmacology (Berl) 234:2883-2895.

Yritia M, Riba J, Ortuño J, Ramirez A, Castillo A, Alfaro Y, de la Torre R, and Barbanoj MJ (2002) Determination of N,N-dimethyltryptamine and betacarboline alkaloids in human plasma following oral administration of Ayahuasca. J Chromatogr B Analyt Technol Biomed Life Sci 779:271-281.

Yu B, Becnel J, Zerfaoui M, Rohatgi R, Boulares AH, and Nichols CD (2008) Serotonin 5-hydroxytryptamine(2A) receptor activation suppresses tumor necrosis factor-alpha-induced inflammation with extraordinary potency. $J$ Pharmacol Exp Ther 327:316-323.

Yuan L, Liu S, Bai X, Gao Y, Liu G, Wang X, Liu D, Li T, Hao A, and Wang Z (2016) Oxytocin inhibits lipopolysaccharide-induced inflammation in microglial cells and attenuates microglial activation in lipopolysaccharide-treated mice. J Neuroinflammation 13:77.

Yubero-Lahoz S, Pardo R, Farré M, O’Mahony B, Torrens M, Mustata C, Pérez-Mañá C, Carbó ML, and de la Torre R (2011) Sex differences in 3,4-methylenedioxymethamphetamine (MDMA; ecstasy)-induced cytochrome P450 2D6 inhibition in humans. Clin Pharmacokinet 50:319-329.

Zanos P, Nelson ME, Highland JN, Krimmel SR, Georgiou P, Gould TD and Thompson SM (2017) A negative allosteric modulator for $\alpha 5$ SubunitContaining GABA receptors exerts a rapid and persistent antidepressant-like action without the side effects of the NMDA receptor antagonist ketamine in mice. eNeuro 4:1-11.

Zarate CA Jr and Manji HK (2008) The role of AMPA receptor modulation in the treatment of neuropsychiatric diseases. Exp Neurol 211:7-10.

Zarate CA Jr, Singh JB, Carlson PJ, Brutsche NE, Ameli R, Luckenbaugh DA Charney DS, and Manji HK (2006) A randomized trial of an N-methyl-D-aspartate antagonist in treatment-resistant major depression. Arch Gen Psychiatry 63: 856-864.

Zeifman RJ, Singhal N, Dos Santos RG, Sanches RF, de Lima Osório F, Hallak JEC, and Weissman CR (2020) Rapid and sustained decreases in suicidality following a single dose of ayahuasca among individuals with recurrent major depressive disorder: results from an open-label trial. Psychopharmacology (Berl), doi: 10.1007/ s00213-020-05692-9.

Zeifman RJ, Palhano-Fontes F, Hallak J, Arcoverde E, Maia-Oliveira JP, and Araujo DB (2019) The impact of ayahuasca on suicidality: results from a randomized controlled trial. Front Pharmacol 10:1325.

Zhang C and Marek GJ (2008) AMPA receptor involvement in 5hydroxytryptamine $2 \mathrm{~A}$ receptor-mediated pre-frontal cortical excitatory synaptic 
currents and DOI-induced head shakes. Prog Neuropsychopharmacol Biol Psychiatry 32:62-71.

Zhang L, Benedek DM, Fullerton CS, Forsten RD, Naifeh JA, Li XX, Hu XZ, Li H, Jia $\mathrm{M}$, Xing GQ, et al. (2014) PTSD risk is associated with BDNF Val66Met and BDNF overexpression. Mol Psychiatry 19:8-10.

Zhang L and Jones EG (2004) Corticothalamic inhibition in the thalamic reticular nucleus. J Neurophysiol 91:759-766.

Zhang Y, Llinas RR, and Lisman JE (2009) Inhibition of NMDARs in the nucleus reticularis of the thalamus produces delta frequency bursting. Front Neural Cir cuits 3:20.

Zhao ZY, Castagnoli N Jr, Ricaurte GA, Steele T, and Martello M (1992) Synthesis and neurotoxicological evaluation of putative metabolites of the serotonergic neurotoxin 2-(methylamino)-1-[3,4-(methylenedioxy)phenyl] propane [(methylenedioxy)methamphetamine]. Chem Res Toxicol 5:89-94.
Zhou Z-S and Zhao Z-Q (2000) Ketamine blockage of both tetrodotoxin (TTX)-sensitive and TTX-resistant sodium channels of rat dorsal root ganglion neurons. Brain Res Bull 52:427-433.

Zhu LX, Sharma S, Gardner B, Escuadro B, Atianzar K, Tashkin DP, and Dubinett SM (2003) IL-10 mediates sigma 1 receptor-dependent suppression of antitumor immunity. J Immunol 170:3585-3591.

Zorn JV, Schür RR, Boks MP, Kahn RS, Joëls M, and Vinkers CH (2017) Cortisol stress reactivity across psychiatric disorders: a systematic review and metaanalysis. Psychoneuroendocrinology 77:25-36.

Zorumski CF, Izumi Y, and Mennerick S (2016) Ketamine: NMDA receptors and beyond. J Neurosci 36:11158-11164.

Zou W, Feng R, and Yang Y (2018) Changes in the serum levels of inflammatory cytokines in antidepressant drug-naïve patients with major depression. PLoS One 13:e0197267. 